SLAC-R-843

September 2006

\title{
Papers from U.S. Department of Energy Science Undergraduate Laboratory Internship \\ Program (SULI) 2006
}

Editors: A. Edwards, S. Majewski and M. Woods

SLAC-R-843

Prepared for the Department of Energy

under contract number DE-AC02-76SF00515

Printed in the United States of America. Available from the National Technical Information Service, U.S. Department of Commerce, 5285 Port Royal Road, Springfield, VA 22161. 
This document, and the material and data contained therein, was developed under sponsorship of the United States Government. Neither the United States nor the Department of Energy, nor the Leland Stanford Junior University, nor their employees, nor their respective contractors, subcontractors, or their employees, makes an warranty, express or implied, or assumes any liability of responsibility for accuracy, completeness or usefulness of any information, apparatus, product or process disclosed, or represents that its use will not infringe privately owned rights. Mention of any product, its manufacturer, or suppliers shall not, nor is it intended to, imply approval, disapproval, or fitness of any particular use. A royalty-free, nonexclusive right to use and disseminate same of whatsoever, is expressly reserved to the United States and the University. 


\title{
Simulation of the BaBar Drift Chamber
}

\author{
Rachel Anderson \\ Office of Science, Science Undergraduate Laboratory Internship (SULI) \\ University of Wisconsin - Eau Claire \\ Stanford Linear Accelerator Center \\ Stanford, CA
}

August 24, 2006

Prepared in partial fulfillment of the requirements of the Office of Science, Department of Energy's Science Undergraduate Laboratory Internship under the direction of Michael Kelsey and Jochen Dingfelder at the Experimental Group C, Stanford Linear Accelerator Center.

Participant:

Signature

Research Advisor:

\author{
Signature
}




\section{TABLE OF CONTENTS}

$\begin{array}{ll}\text { Abstract } & \text { ii }\end{array}$

$\begin{array}{ll}\text { Introduction } & 1\end{array}$

Materials and Methods $\quad 4$

$\begin{array}{lr}\text { Results } & 6\end{array}$

$\begin{array}{ll}\text { Discussion and Conclusions } & 7\end{array}$

$\begin{array}{lr}\text { Acknowledgments } & 8\end{array}$ 


\begin{abstract}
Simulation of the BaBar Drift Chamber. RACHEL ANDERSON (University of Wisconsin - Eau Claire, Eau Claire, WI 54701) MICHAEL KELSEY AND JOCHEN DINGFELDER (Experimental Group C, Stanford Linear Accelerator Center, Stanford, CA 94025)
\end{abstract}

The BaBar drift chamber (DCH) is used to measure the properties of charged particles created from $e^{+} e^{-}$collisions in the PEP-II asymmetric-energy storage rings by making precise measurements of position, momentum and ionization energy loss $(d E / d x)$. In October of 2005 , the PEP-II storage rings operated with a luminosity of $10 \times 10^{33} \mathrm{~cm}^{-2} \mathrm{~s}^{-1}$; the goal for 2007 is a luminosity of $20 \times 10^{33} \mathrm{~cm}^{-2} \mathrm{~s}^{-1}$, which will increase the readout dead time, causing uncertainty in drift chamber measurements to become more significant in physics results. The research described in this paper aims to reduce position and $d E / d x$ uncertainties by improving our understanding of the BaBar drift chamber performance. A simulation program - called GARFIELD - is used to model the behavior of the drift chamber with adjustable parameters such as gas mixture, wire diameter, voltage, and magnetic field. By exploring the simulation options offered in GARFIELD, we successfully produced a simulation model of the BaBar drift chamber. We compared the time-to-distance calibration from BaBar to that calculated by GARFIELD to validate our model as well as check for discrepancies between the simulated and calibrated time-to-distance functions, and found that for a $0^{\circ}$ entrance angle there is a very good match between calibrations, but at an entrance angle of $90^{\circ}$ the calibration breaks down. Using this model, we also systematically varied the gas mixture to find one that would optimize chamber operation, which showed that the gas mixture of 80:20 Helium:isobutane is a good operating point, though more calculations need to be done to confirm that it is the optimal mixture. 


\section{INTRODUCTION}

The primary goal of the BaBar experiment [1] is to study particles and their antiparticles in order to understand the predominance of matter in the universe. More specifically, the purpose of BaBar is the study of $B^{0}$ and $\bar{B}^{0}$ meson decays to $\mathrm{CP}$ eigenstates so that we may understand CP-violating asymmetries. Further goals of BaBar are to identify and perform comprehensive studies of beauty and charm mesons, and tau leptons.

Inside the BaBar detector, the $9.1 \mathrm{GeV}$ electron beam and the $3 \mathrm{GeV}$ positron beam of the PEP-II storage rings collide with a center-of-mass energy equal to the mass of the $\Upsilon(4 \mathrm{~S})$ resonance $(10.58 \mathrm{GeV})$. The $\Upsilon(4 \mathrm{~S})$ is a $b \bar{b}$ bound state which decays with equal probability into $B^{+} B^{-}$or $B^{0} \bar{B}^{0}$ mesons creating the ideal situation for studying $B^{0}$ and $\bar{B}^{0}$ meson decays.

The BaBar detector is a cylindrical detector consisting of a silicon vertex tracker (SVT), surrounded by the drift chamber (DCH), a Cherenkov Detector (DIRC), then a cesium iodide (CsI) crystal calorimeter (EMC) wrapped in the Instrumented Flux Return (IFR). Around all of this is a superconducting solenoid which produces a magnetic field of $1.5 \mathrm{~T}$ along the length of the chamber, the $z$ direction. The BaBar detector reconstructs the paths of particles as they traverse the chamber, and determines their momenta. Cherenkov light from the particle's interaction in quartz bars in the DIRC provides a velocity measurement. Combined with the momentum measurement from the DCH, we can identify the different mass of the particles. Hadrons, which generally do not interact in the EMC, are filtered in the IFR for muon identification by measuring the momentum and specific ionization loss, $d E / d x$, using the resulting data to calculate the mass, then matching the mass to the corresponding particle.

The charged particles produced in the $e^{+}$and $e^{-}$collisions (such as $B, D$, or $\tau$ ) traverse the detector, ionizing the gas. The displaced electrons drift towards the sense wire, causing an electrical pulse on the wire telling us not only the wire the particle's trajectory is close to, but 
the time since the event was triggered (a combination of hardware and software signal which indicate that an interesting event occurred). Using a time-to-distance calibration (shown in Figure 1), we can use the time since the trigger to determine how close the particle was to the sense wire. From this information, the particle's distance from each sense wire it passed near is calculated, and the reconstruction process determines the path of the particle and the momentum.

The PEP-II accelerator operates at a luminosity of $10 \times 10^{33} \mathrm{~cm}^{-2} \mathrm{~s}^{-1}$, such that it is capable of distinguishing about 3,000 events per second. However, by 2007 the BaBar team aims to be operating with a PEP-II luminosity of $20 \times 10^{33} \mathrm{~cm}^{-2} \mathrm{~s}^{-1}$. In order to operate at this luminosity, the DCH must be capable of processing a greater density of events. A hindering factor is the dead time - inefficiency caused by the detectors inability to process an event because it is processing a preceding event. With the increase in luminosity, dead time will become a greater problem as not only will the event rate increase, but background noise as well.

In order to distinguish the small electrical pulses generated by charged particles passing by the DCH sense wires from other signals and background noise, the chamber must operate with a high drift velocity: the average velocity at which an ionization electron travels through a gas. This will not only decrease the dead time, but cause the ionization electrons to travel in a straighter path leading to a stronger distance to arrival time correlation. For the distance we used the distance of closest approach (DOCA), shown in Figure 2, as it is at that distance that we will get the minimum drift time. For the same reason, it is important to keep the Lorentz angle at a minimum, as it will help in spatial resolution. The Lorentz angle is the angle of the drift trajectory to the magnetic field. As the Lorentz angle increases, electron drift path curvature also increases. Finally, we want to maximize $d E / d x$ (specific energy lost due to collisions with gas molecules) for a stronger pulse and a greater resolution.

The goal of our research is to create an accurate computer simulation of the BaBar drift chamber using a software program called GARFIELD. Once a model was established, we used 
GARFIELD to fulfill the following:

- Vary the entrance angle to study its impact on drift time.

- Systematically vary the gas mixture in order to explore possible solutions that might promote a higher drift velocity, while keeping a large $d E / d x$, good $d E / d x$ resolution, and minimizing the Lorentz angle.

- Compare the $t(D O C A)$ correlation from BaBar to that simulated by GARFIELD.

This model will be used to validate the performance of the BaBar DCH, improve the chamber calibrations, and explore variations of chamber settings that could improve chamber operation.

GARFIELD [5] is a computer program that simulates gaseous detectors, given the complete detector definitions including gas composition, magnetic field, voltage, particle definition, and physical components. The program generates plots, histograms, and tables with electric field contours, drift velocity, Townsend coefficient (the number of electron-ion pairs formed by an electron along path of $1 \mathrm{~cm}$ length), diffusion coefficients (rate of electrons drifting perpendicular to electric field), Lorentz angles, as well as information on ionization clusters including their energy and the path they follow through the chamber [3]. GARFIELD can generate an arrival time histogram as well as a plot showing the relationship between the distance of the particles from the sense wire and arrival time. This data can be compared to information from the BaBar DCH to either validate its behavior or find where there might be a discrepancy.

This paper presents comparisons of the BaBar drift chamber to the GARFIELD model, as well as describes the results of varying parameters in the computer simulation. 


\section{MATERIALS AND METHODS}

The BaBar drift chamber is composed of 7104 individual hexagonal cells made up of a tungsten-rhenium sense wire surrounded by a hexagon $120 \mu \mathrm{m}$ gold-plated aluminum field wires. The cells are arranged into 40 axial and stereo layers, sectioned into 10 superlayers, layers 1 through 16 being shown on the left in Figure 3 [1].

We used GARFIELD to model a section of the fourth superlayer of the DCH, also shown in Figure 3. Consistent with the BaBar DCH, we specified a sense wire diameter of $20 \mu \mathrm{m}$ and a field wire diameter of $120 \mu \mathrm{m}$, both with a length of $275 \mathrm{~cm}$. The sense wires have a tension of $30 \mathrm{~g}$ while the field wires have a tension of $190 \mathrm{~g}$. The field wires were grounded, and the sense wires had a voltage of $1930 \mathrm{~V}$. The magnetic field was set in the $+z$ direction with an amplitude of $1.5 \mathrm{~T}$. The drift gas mixture was set to $80 \% \mathrm{He}$ and $20 \% \mathrm{iC}_{4} \mathrm{H}_{10}$ with $3500 \mathrm{ppm} \mathrm{H}_{2} \mathrm{O}$ and 80 ppm $\mathrm{O}_{2}$. For the simulated particle that traverses the modeled fourth superlayer, we specified a positive pion $\left(\pi^{+}\right)$with a kinetic energy of $500 \mathrm{MeV}$. Within the GARFIELD program, we used a program called HEED [4] to simulate the ionization of the gas molecules by a pion to create clusters. We asked that the drift electrons with enough energy to cause secondary ionizations (i.e. delta electrons) be taken into account. For the calculations and plots, we focused on an area of $2.8 \mathrm{~cm}$ by $2.8 \mathrm{~cm}$ centered on sense wire

number four of layer 15, located near the center of the superlayer, in order to avoid fringing effects. It was specified that the electrons start to drift from the surface of the sense wire, in reverse, as though they had a positive charge. In this way the drift line plots show the origin of all electrons that eventually hit the wire, as shown in Figure 4. Within GARFIELD, a program called MAGBOLTz [2] is used to calculate drift velocity and Lorentz angles for electrons in gas mixtures under the influence of electric and magnetic fields.

The different parameters and their values for our most accurate model of the DCH are shown in Table 1. We were able to use GARFIELD to generate histograms, plots, and tables presenting the simulation of the BaBar drift chamber. GARFIELD output includes a print of 
the section of the wire configuration (Figure 3), a contour plot of the electric field, and a plot of electron drift lines (Figure 4). GARFIELD also plots histograms of clustering information, including the distance between electron and track, number of electrons per cluster, number of clusters on the track, distance between cluster and track, energy per cluster, energy lost, and number of electrons on the track. From the timing section, GARFIELD plots histograms for both the arrival time of the earliest electron on the sense wire (Figure 5) and the arrival time of all electrons. For the gas study, GARFIELD's output includes graphs of the drift velocity, diffusion coefficients (both transverse and longitudinal), Townsend coefficient, attachment coefficient, and Lorentz angle (Figure 5) all with respect to the electric field. With our DCH model, we are able to use this data to learn more about the BaBar DCH.

Our first study was on the effect of the entrance angle on drift velocity. We used GARFIELD to generate clusters of electrons on points $0.5 \mathrm{~cm}$ from the sense wire. We then rotated from $-180^{\circ}$ to $180^{\circ}$ in 100 steps around the sense wire, each time calculating the angle and the drift time of an electron starting at that point. While HEED generates a more physical clustering model, these fixed points were a simplification that we were able to use to compare the drift velocity of electrons starting on tracks $0^{\circ}$ to $360^{\circ}$ from the $r$-axis (Figure 2).

For the second goal of this research, to find a better system design, we used the chamber model and varied the helium-isobutane gas mixture from 100:0 to 70:30 to explore the effects of isobutane. Although a gas mixture of 100:0 would be disastrous for the chamber (allows discharges at low voltages), it is used as a comparison. We compared the drift time, Lorentz angle (both in Figure 5), and $d E / d x$ (Figure ??) for the different gas mixtures. We computed the drift time from GARFIELD's timing command, looking at the fastest electron to reach the sense wire from a track with a DOCA of $0.8 \mathrm{~cm}$ (Figure 7). For this, GARFIELD used 1000 iterations, of which we used the fastest. The Lorentz angles are compared in Figure 5. Finally, to study the difference in specific ionization energy loss, we used the total-energylost clustering-histogram from the drift section of GARFIELD. For this calculation, GARFIELD also used 1000 iterations, from which we computed an 80\% truncated mean (the smallest 
$80 \%$ of results). The track we used was $1.6 \mathrm{~cm}$ in length, and had a DOCA of $0.48 \mathrm{~cm}$

(Figure 7), as shown in Figure 7. From these comparisons we were able to determine the optimal gas mixture from among those studied.

With this DCH model established, we compared it to the BaBar DCH. Using GARFIELD we generated a plot of the distance between a particle and a sense wire with respect to time. To do this, we considered tracks with entrance angles $\psi$ from the sense wire, then rotated it from $0^{\circ}$ to $90^{\circ}$ in increments of $5^{\circ}$. Fore each $\psi$ we computed the drift time for tack DOCAs spanning the cell in $0.1 \mathrm{~cm}$ steps. From this data we made a time-to-distance plot which we compared to the corresponding calibration used by the BaBar reconstruction software.

\section{RESULTS}

Figure 8 displays the impact that entrance angle can have on drift velocity. Though the overall velocity only changes within $0.9 \mathrm{~ns}$, it does show a sinusoidal relation, shown by the graph on the left. The plot on the right shows the time to DOCA relationship for entrance angles of $-30^{\circ}, 0^{\circ}, 30^{\circ}$, and $90^{\circ}$. Near the sense wire $(\mathrm{DOCA}=0 \mathrm{~cm})$ the electric field is symmetric, so all curves look the same. However, near the field wires, where the electric field is spatially asymmetric, the drift velocities vary and the shape of the $t(D O C A)$ curves change.

The results of the gas study show the importance of the balance between arrival time, Lorentz angle, and $d E / d x$. As the percentage of isobutane increased, the drift velocity decreased as there were more interactions between particles, shown by the histogram in Figure 5. For the drift velocity, it appears that the 70:30 gas mixture is the best choice as it has the smallest arrival time. However, the Lorentz angle graph shows that as the isobutane increases, so does the Lorentz angle for BaBar's operating voltage of $1930 \mathrm{~V}$. For the 70:30 gas mixture, the Lorentz angle is greatest, while that for the 80:20 mixture is better. The Lorentz angle for the 90:10 mixture is the best, however due to its higher drift time it would 
not be the optimal choice. It is left to $d E / d x$ to decide between the 80:20 and the 70:30.

The final part of the gas study was the comparison between $d E / d x$ for different gas mixtures. From the graphs in Figure 6, we see that as isobutane increases, $d E / d x$ also increases, as isobutane is a quencher and neutralizes the ions. Once again the 70:30 gas mixture appears to be the best, as it has the desired maximum $d E / d x$. Finally, the $d E / d x$ resolution plot shown on the right in Figure 6 shows that the 70:30 gas mixture also has the best resolution. However, as isobutane is highly flammable, the more isobutane is in the mixture, the more hazardous it would be to work with. It is the balance between arrival time, Lorentz angle, and $d E / d x$ that leads to the conclusion that the 80:20 gas mixture used in BaBar is a good operating point as it keeps the Lorentz angle lower. The difference in Lorentz angles for the 80:20 and the 70:30 gas mixtures is approximatley $2^{\circ}$, therefore, if we were to change the gas mixture our results say that the 70:30 mixture would be ideal.

The results of the time-to-distance calibration comparison are shown in Figure 1. For an entrance angle of $0^{\circ}$ (particle is passing straight through the chamber), the GARFIELD simulation is a very good match. For the $30^{\circ}$ and $-30^{\circ}$ entrance angles, there is a clear discrepancy between the GARFIELD prediction and BaBar's calibration. Finally, for an entrance angle of $90^{\circ}$ (particle is traveling in a loop through the chamber), the BaBar calibration breaks down noticeably.

\section{DISCUSSION AND CONCLUSIONS}

We were able to successfully model the performance of the BaBar drift Chamber. The arrival time vs. entrance angle study showed that near the sense wire the different time-to-distance correlations from different entrance angles behaves the same, as the electric field is symmetric. However, near the field wires, where the electric field is spatially asymmetric the $t(D O C A)$ curves vary significantly with the entrance angle. From the gas study, it was shown that a gas mixture of 80:20 Helim:Isobutane is a good operating point. The 80:20 mixture is a good 
balance between the arrival time, the Lorentz angle and $d E / d x$. While the 70:30 mixture has the minimum arrival time, and the best $d E / d x$ resolution, its Lorentz angle is greater than that for the 80:20 mixture. Balancing these three, the 80:20 mixture that the BaBar DCH uses appears to be a good operating point. However, were the gas mixture to be changed, a 70:30 isobutane mixture is recommended. For the time-to-distance calibration comparison, we found a good agreement between the calibrated real data and the simulation for $0^{\circ}$, but found that the calibrations for non-zero entrance angles have systematic discrepancies which could be affecting reconstruction.

Future studies include understanding the discrepancy between the BaBar time-to-distance calibration and the GARFIELD simulation, as well as fixing those discrepancies which are negatively affecting reconstruction, in order to improve tracking efficiency. Furthermore, now that a computer simulation for the BaBar DCH has been constructed, future studies can be done to explore variations of chamber settings that could improve operation.

\section{ACKNOWLEDGMENTS}

The author would like to thank Michael Kelsey, Jochen Dingfelder, and Adam Boyarski from Experimental Group C for your guidance, as well as Rob Veenhof for help with GARFIELD,

and the U.S. Department of Energy Science Undergraduate Laboratory Internship Program for the opportunity to work at SLAC. 


\section{REFERENCES}

[1] BaBar Collaboration, The BaBar detector, Nuclear Instruments and Methods in Physics Research A 479, 1-116 (2005).

[2] Biagi, Stephen, Magboltz: Transport of electrons in gas mixtures, http://consult.cern.ch/writeup/magboltz, (2005).

[3] Kleinknecht, Konrad, Detectors for particle radiation, Cambridge University Press, Cambridge (1987).

[4] Smirnov Igor, Heed: Interactions of particles with gases, CERN Computer Newsletter $226,13(1996)$.

[5] Veenhof, Rob, Garfield - simulation of gaseous detectors, CERN Computer News Letter 2000-001 Vol. XXXV, issue no 1 (2000). 


\section{FIGURES}
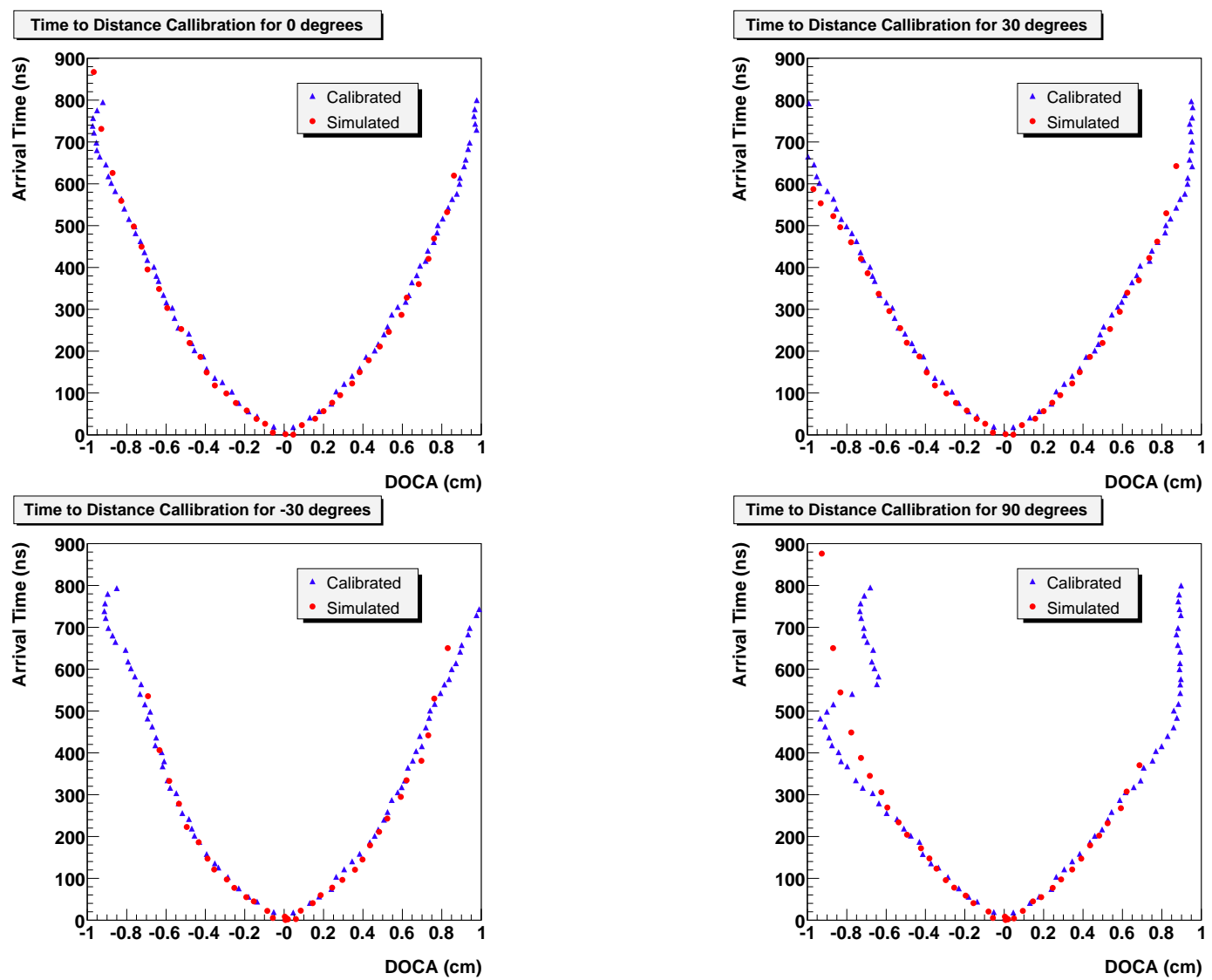

Figure 1: time-to-distance calibration for the DCH: GARFIELD's simulation (red circles) vs. BaBar's time-to-distance calibration (blue triangles) for four different entrance angles $\left(-30^{\circ}, 0^{\circ}, 30^{\circ}, 90^{\circ}\right)$. 


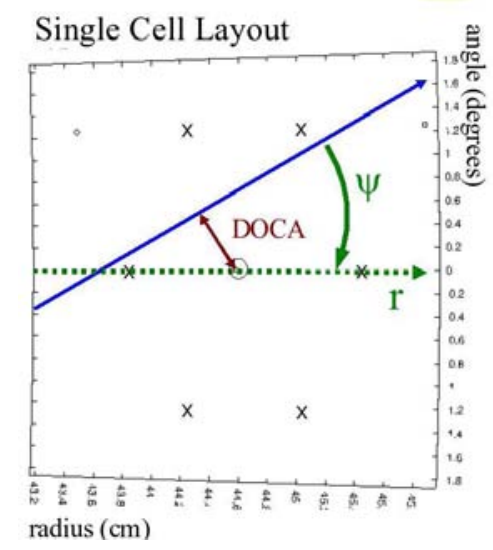

Figure 2: Particle track distance of closest approach (DOCA) and entrance angle, $\psi \cdot \mathbf{r}$ is the vector from center of detector through sense wire.
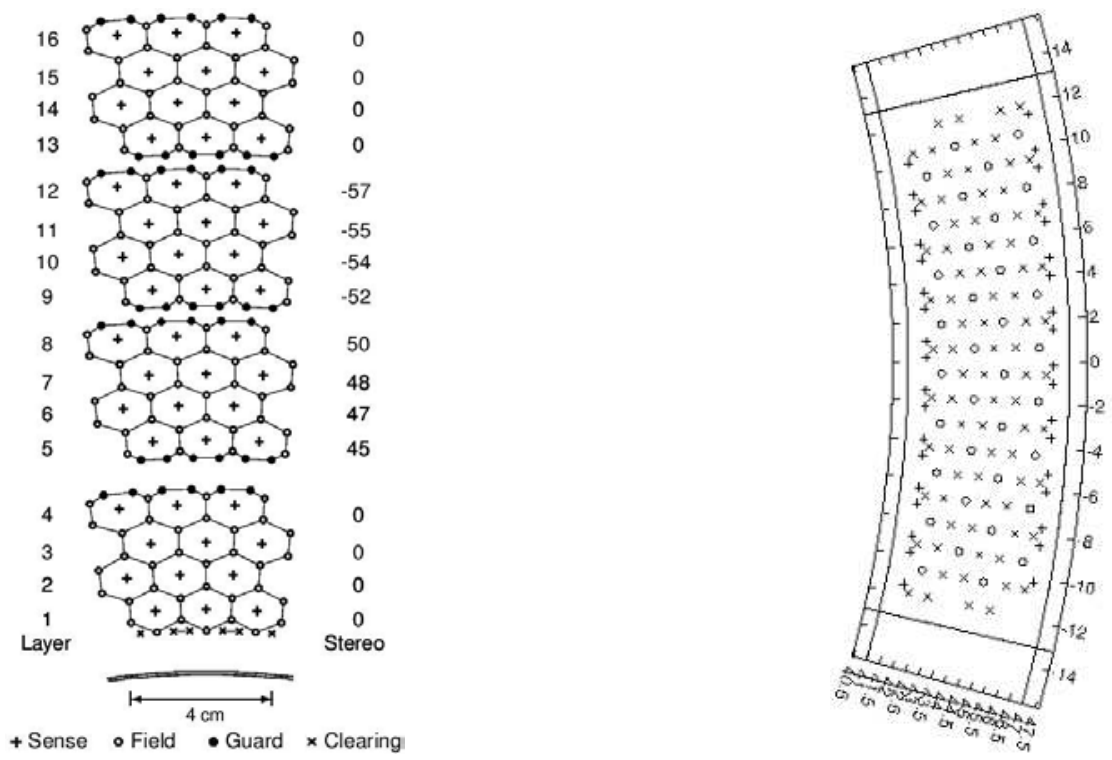

Figure 3: On the left is the wire configuration for BaBar DCH section. On the right is the cell entered into GARFIELD drift chamber model, with $(\mathbf{r}, \phi)$ coordinates shown. Sense wires are shown by circles, field wires are shown by the x's, and guard wires are crosses. 


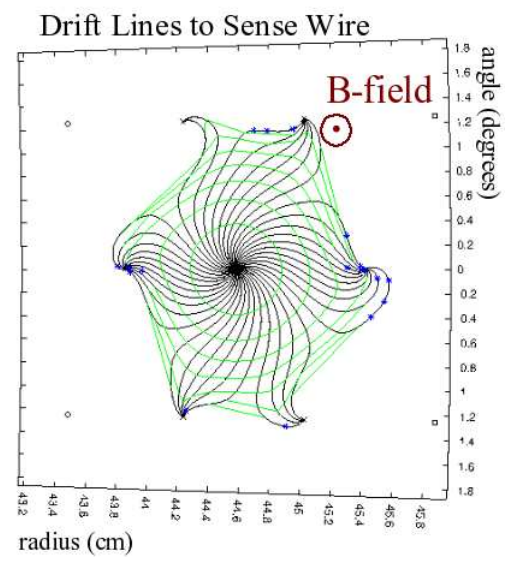

Figure 4: Drift lines and isochrones around sense wire, with $1.5 \mathrm{~T}$ magnetic field.
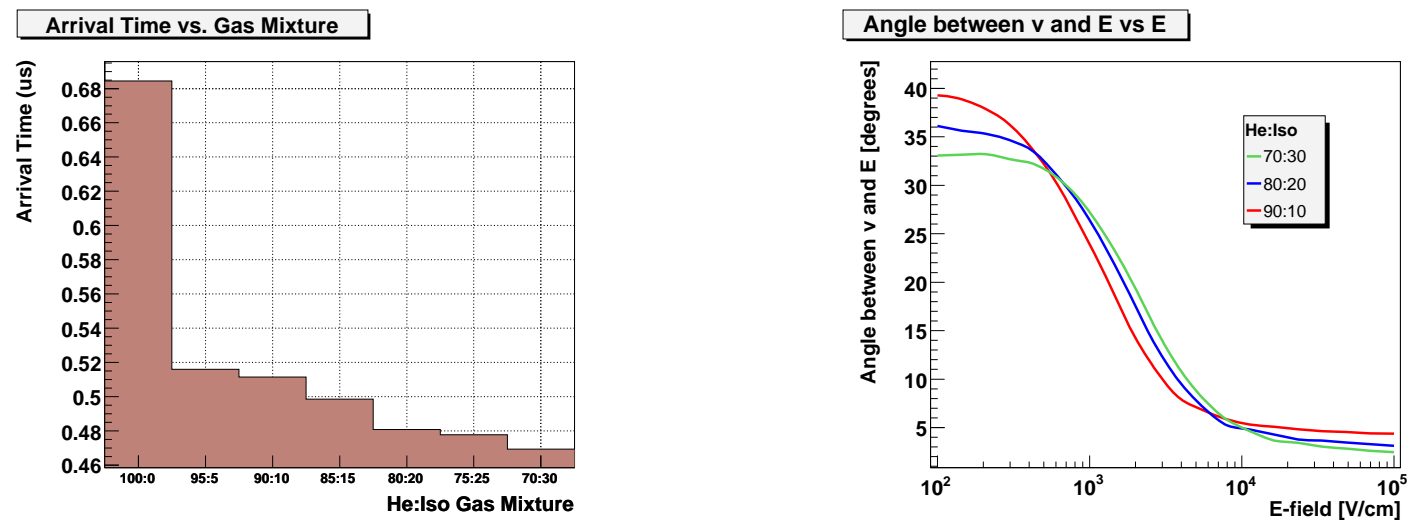

Figure 5: Histogram on left shows the arrival time for different Helium:Isobutane proportions, where 80:20 is BaBar's gas mixture. Graph on the right shows the Lorentz angle as a function of electric field (for a magnetic field of $1.5 \mathrm{~T}$ ). BaBar operates with an electric field of $2 \times 10^{3} \mathrm{~V} / \mathrm{cm}$.
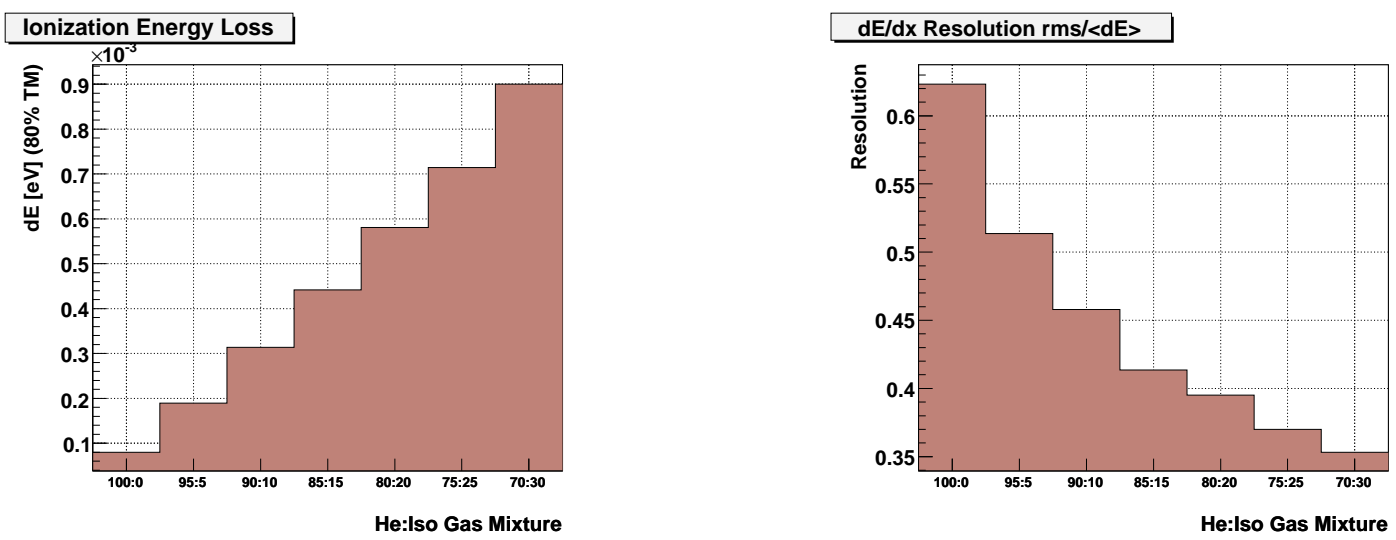

Figure 6: Histogram on left shows $d E / d x$ for different gas mixtures from He:Iso 100:0 to 70:30, where 80:20 is BaBar's operating point. The histogram on the right shows the $\mathrm{dE} / \mathrm{dx}$ resolution, $\mathrm{RMS}(\mathrm{dE} / \mathrm{dx}) / \mathrm{idE} / \mathrm{dx} \dot{i}$, for the test particle. 

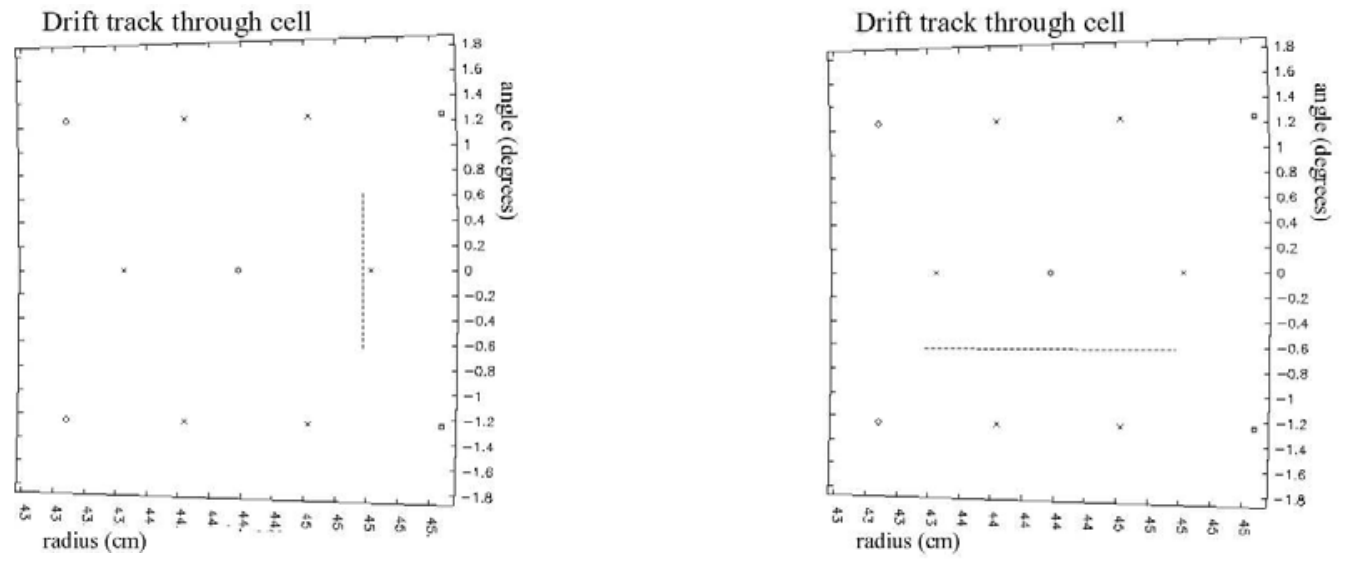

Figure 7: On the left is the track that the arrival time was calculated from. On the right is the track on which $d E / d x$ is calculated.
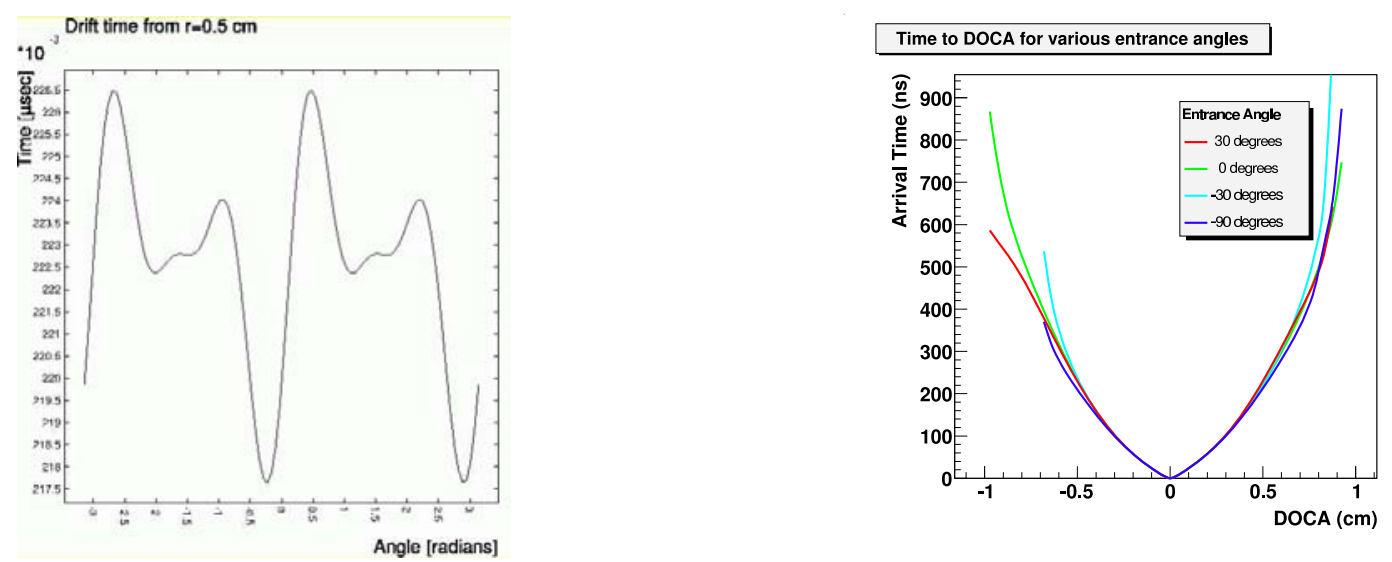

Figure 8: Drift time vs. entrance angle of electron. The graph on the left is of a particle starting a distance of $0.5 \mathrm{~cm}$ from the sense wire for all entrance angles. The graph on the right is of particles starting between $-1 \mathrm{~cm}$ and $1 \mathrm{~cm}$ from the sense wire at entrance angles $-90^{\circ},-30^{\circ}, 0^{\circ}$, and $30^{\circ}$. 


\section{TABLES}

\begin{tabular}{|l|c|}
\hline Parameters & Value \\
\hline sense wire diameter & $0.0020 \mathrm{~cm}$ \\
field wire diameter & $0.0120 \mathrm{~cm}$ \\
wire length & $275 \mathrm{~cm}$ \\
sense wire voltage & $1930 \mathrm{~V}$ \\
field wire voltage & $0 \mathrm{~V}$ \\
sense wire density & $106.95 \mathrm{~g} / \mathrm{cm}^{3}$ \\
field wire density & $14.94 \mathrm{~g} / \mathrm{cm}^{3}$ \\
sense wire tension & $30 \mathrm{~g}$ \\
field wire tension & $190 \mathrm{~g}$ \\
magnetic field & $1.5 \mathrm{~T}$ \\
$\mathrm{He}$ & $80 \%$ \\
$\mathrm{iC}_{4} \mathrm{H}_{10}$ & $20 \%$ \\
$\mathrm{H}_{2} \mathrm{O}$ & $3500 \mathrm{ppm}$ \\
$\mathrm{O}_{2}$ & $80 \mathrm{ppm}$ \\
\hline
\end{tabular}

Table 1: The table shows the variables and their values as entered into GARFIELD. 


\title{
A Catalog of Candidate High-redshift Blazars for GLAST
}

\author{
Tersi M. Arias \\ Office of Science, Science Undergraduate Laboratory Internship (SULI) \\ San Francisco State University \\ Stanford Linear Accelerator Center \\ Menlo Park, California
}

Aug 25, 2006

Prepared in partial fulfillment of the requirements of the Office of Science, U.S. Department of Energy's Science Undergraduate Laboratory Internship (SULI) Program under the direction of

J. Carson in the Kavli Institute of Particle Astrophysics and Cosmology at Stanford Linear Accelerator Center.

Participant:

Signature

Research Advisor:

\section{Signature}




\section{Table of Contents}

\section{Page}

Abstract

iii

Introduction

1

Methods

2

Results

Discussion and Conclusions

4

Acknowledgments

5

References

Tables

6

Figures

$6-8$ 


\begin{abstract}
A Catalog of Candidate High-redshift Blazars for GLAST. TERSI ARIAS (San Francisco State University, San Francisco, CA 94132) JENNIFER CARSON (Stanford Linear Accelerator Center, Menlo Park, CA 94025)
\end{abstract}

High-redshift blazars are promising candidates for detection by the Gamma-ray Large Area Space Telescope (GLAST). GLAST, expected to be launched in the Fall of 2007, is a highenergy gamma-ray observatory designed for making observations of celestial gamma-ray sources in the energy band extending from $10 \mathrm{MeV}$ to more than $200 \mathrm{GeV}$. It is estimated that GLAST will find several thousand blazars. The motivations for measuring the gamma-ray emission from distant blazars include the study of the high-energy emission processes occurring in these sources and an indirect measurement of the extragalactic background light. In anticipation of the launch of GLAST we have compiled a catalog of candidate high-redshift blazars. The criteria for sources chosen for the catalog were: high radio emission, high redshift, and a flat radio spectrum. A preliminary list of 307 radio sources brighter than $70 \mathrm{mJy}$ with a redshift $z \geq 2.5$ was acquired using data from the NASA Extragalactic Database. Flux measurements of each source were obtained at two or more radio frequencies from surveys and catalogs to calculate their radio spectral indices $\alpha$. The sources with a flat-radio spectrum $(\alpha \leq 0.5)$ were selected for the catalog, and the final catalog includes about 200 sources. 


\section{INTRODUCTION}

Blazars are active galactic nuclei (AGN) with relativistic plasma jets oriented close to the line of sight of the observer. These objects are intrinsically powerful variable radio sources and are the largest class of identified high-energy gamma-ray sources. Gamma-ray blazars are characterized by rapid flux variability and a high degree of linear polarization at visible wavelengths, with the measured rate of change limited by the statistics of the gamma-ray observations [1].

The study of high-energy emission processes in blazars has been difficult in the past because of the low number of gamma-ray blazars detected, especially at high redshifts. However, The Gamma-ray Large Area Space Telescope (GLAST), scheduled to launch in the Fall of 2007 will easily detect high-energy gamma-ray emission in the GeV range. GLAST will scan the entire sky every three hours and will provide long, evenly sampled, gamma-ray light curves for a large number of sources [2] including blazars. The much greater sensitivity of GLAST with respect to previous instruments should result in a large increase in the number of known highenergy sources, and it is estimated that GLAST will detect several thousand new gamma-ray blazars.

Spectral energy distribution (SED) is defined by power as a function of frequency $\left(v F_{v}\right.$ vs. $\left.v\right)$. The SED of blazars has a characteristic double peak signature, as shown in Figure 1, which suggests that synchrotron radiation and Compton scattering occurs in the jets. Electrons inside the jet are highly relativistic and are moved around by magnetic fields. The energy release ranges from relatively weak radio frequencies to powerful gamma-ray photons. High-energy gamma-ray emission is produced from inverse-Compton scattering of low-energy photons off relativistic electrons. The radio-frequency spectra and polarization properties of blazars are characteristics of synchrotron radiation from relativistic electrons having a power law distribution of electron energies [3]. Over small intervals the SED is characterized by a powerlaw distribution $F_{v} \propto v^{-\alpha}$, where $\alpha$ is the spectral index. A spectral index of $\alpha \leq 0.5$ is considered a flat radio spectrum and is characteristic of blazars. 
The study of absorption features imprinted on the gamma-ray spectra of distant blazars provides information about the extragalactic background light (EBL). The diffuse EBL consists of the sum of the starlight emitted by galaxies through the history of the universe. EBL causes attenuation of gamma-ray photons via pair production when they travel through space. Highredshift blazars should be significantly affected by EBL absorption and the further away they are, the more absorbed the gamma-ray spectrum will be.

This project focuses on gathering information from compact radio sources with high redshifts $(z \geq 2.5)$ and flat spectra $(\alpha \leq 0.5)$ from databases such as the Nasa Extragalactic Database (NED) and surveys such as the combined National Radio Astronomy Observatory (NRAO) and the Very Large Array (VLA) Sky Survey (NVSS). The goal is to build a catalog of

sources that are promising candidates for detection by GLAST. The GLAST measurements of these sources could provide new and extensive information about the extragalactic background light and the history of the universe.

\section{MATERIALS AND METHODS}

A preliminary list of radio sources at high redshift was obtained from the NASA Extragalactic Database (NED). NED is a comprehensive database of extragalactic objects with known positions and redshifts [4]. Two parameters were selected in the search query: radio emission in the $21 \mathrm{~cm}$ band $(1.4 \mathrm{GHz})$ greater than $70 \mathrm{mJy}$, and redshift $z \geq 2.5$. With these parameters, 238 objects where obtained to analyze. To determine that there are not missing objects from the $21 \mathrm{~cm}$ band query, sources with radio emission above $70 \mathrm{mJy}$ at $20 \mathrm{~cm}$ and $6 \mathrm{~cm}$ were also selected. In this way 69 more objects were obtained, for a total of 307 sources for the preliminary list.

Sources with a flat radio spectrum $(\alpha \leq 0.5)$ are desired for the catalog. To determine this kind of spectrum it is necessary to obtain precise measurements of each object's flux in at least two different frequencies and calculate their individual spectral indices. 


\section{Finding Fluxes}

The primary flux measurements of interest were at 1.4GHz. The NRAO/VLA Sky Survey (NVSS) was the main source of this data. NVSS is a survey of the sky using the Very Large Array (VLA) radio telescope at a wavelength of $20 \mathrm{~cm}(1.4 \mathrm{GHz})$ and it covers the northern sky (declination $\delta>-40$ ). Radio flux values at $1.4 \mathrm{GHz}$ frequency were obtained for 291 of the 307 sources in the preliminary list. Two of these objects were determined not to be radio sources.

The remaining 16 fluxes where south of the declination limit in the northern hemisphere surveys, for these 16 we obtained fluxes at $0.843 \mathrm{GHz}$ from the Sydney University Molonglo Sky Survey (SUMSS), a deep radio survey of the entire sky south of $\delta=-30$ degrees, that uses the Molonglo Observatory Synthesis Telescope.

Radio fluxes were also gathered at 4.85, 0.365, and 8.4GHz. Flux measurements at 4.85GHz were found in the 87 Green Bank (87GB) catalog from the VizieR service web page [5] and the Parkes-MIT-NRAO (PMN) surveys. The $87 \mathrm{~GB}$ is a catalog of radio sources covering 6.0 steradians of sky that used the 91m telescope in Green Bank in 1987 and the PMN surveys are source catalogs for equatorial zone. The Texas survey of radio sources provided flux measurements at $0.365 \mathrm{GHz}$. This survey was carried out with the Texas Interferometer during 1974-1983.

We also made use of the radio flux at $8.4 \mathrm{GHz}$, as found by the Cosmic Lens All-Sky Survey (CLASS), to verify spectral index calculations. CLASS is a survey for gravitationally lensed compact radio sources observed with the VLA. Table 1 summarizes the surveys from which detections were obtained.

\section{Finding Spectral indices}

Once the fluxes were obtained the spectral index was calculated between two radio fluxes by using the following formula:

$$
\alpha=\log \left(\frac{F_{2}}{F_{1}}\right) / \log \left(\frac{v_{1}}{v_{2}}\right)
$$


Where $F_{1}$ and $F_{2}$ are the flux values obtained at two different frequencies, and $v_{1}$ and $v_{2}$ are their respective frequencies.

\section{RESULTS}

At least two flux measurements were found for 305 of the 307 objects of the preliminary list. This allowed the calculation of all but two of the spectral indices of all the objects from the preliminary list. All the data obtained for each source was tabulated.

Table 2 displays a sample of the table of the 307 sources with positions, redshifts, flux values at 5 different frequencies, and their calculated spectral index or indices if they had more then two flux values. Spectral indices were calculated for the following flux ratios: $F_{4.85 \mathrm{GHz}} / F_{1.4 \mathrm{Ghz}}$, $F_{4.85 \mathrm{GHz}} / F_{0.843 \mathrm{GHz}}, F_{1.4 \mathrm{GHz}} / F_{0.365 \mathrm{GHz}}$, and $F_{8.4 \mathrm{GHz}} / F_{1.4 \mathrm{GHz}}$.

Figure 1 shows the SED of a well-studied blazar 3C279. Figure 2 and 3 are flux vs. spectral index distribution plots at different frequencies. Of the 305 sources 215 had a spectral index of $\alpha \leq 0.5$. Two additional sources with $\alpha \leq 0.7$ were characterized in the literature as blazars so were also included in the flat-spectrum sample. Figure 4 shows the redshift distribution of the 217 flat-radio spectrum sources from our catalog.

\section{DISCUSSION AND CONCLUSION}

The ultimate goal of this project was to compile a catalog of high-redshift blazars to be found by GLAST. The calculation of the spectral index of each individual object was needed in order to identify blazars from the preliminary list. Because the positions of the sources varied in the sky, it was needed to find as many flux values in different frequencies as possible to have at least two flux measurements for each source to obtain their spectral indices. Only two spectral indices could not be calculated and we succeeded in identifying 217 objects as flat-radio spectrum sources. 


\section{ACKNOWLEDGEMENTS}

I would like to thank my mentor, Jennifer Carson, for her great guidance, dedication, and patience through my learning process. I also thank Teddy Cheung for teaching me the use of databases, surveys, and catalogs from the web and making sure I had all the resources to complete this project. I thank the U.S. Department of Energy, Office of Science for the opportunity I was given to participate in the SULI program and have such an important learning experience.

\section{REFERENCES}

[1] D. J. Thompson "Blazar Variability: A Gamma-Ray perspective" Blazar Variability Workshop II: Entering the GLAST Era ASP Conference Series, Vol. 350, 2006

[2] H. R. Miller, K. Marshall, J. R. Webb, and M. F. Aller "The Capabilities of the GLAST LAT for Studies of BlazarVariability" in ASP Conference Series, Vol. 350, 2006

[3] G.L. Verschuur, K.I. Kellermann. "Galactic and Extragalactic Radio Astronomy" Springer-Verlag second edition 1988.

[4] NASA Extragalactic Database. http://nedwww.ipac.caltech.edu/index.html

[5] VIzieR service webpage. http://vizier.u-strasbg.fr/viz-bin/VizieR 


\begin{tabular}{|l|r|r|}
\hline & & \\
Survey & Frequency $(\mathrm{GHz})$ & Number of Objects \\
\hline NVSS & 1.1 & 291 \\
SUMSS & 0.843 & 30 \\
87GB & 4.85 & 205 \\
PMN & 4.85 & 66 \\
Texas & 0.365 & 183 \\
& & \\
\hline
\end{tabular}

Table 1. Number of objects with detected flux at 4.85, 1.4, 0.843 , and $0.365 \mathrm{GHZ}$

\begin{tabular}{|c|c|c|c|c|c|c|c|c|c|c|c|c|}
\hline Object Name & $\mathrm{RA} \& \mathrm{DE} \mathrm{C}_{\text {postion: }}$ & & 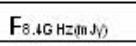 & 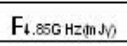 & F8เзмнд(n) & 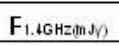 & 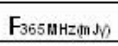 & $z$ & $\begin{array}{c}\boldsymbol{\alpha} \\
4.85 \mathrm{GHZ} / 1.4 \mathrm{GHz} \\
\end{array}$ & $\begin{array}{c}\boldsymbol{\alpha} \\
1.4 \mathrm{GHZ} 365 \mathrm{MHz}\end{array}$ & $\begin{array}{c}\mathbf{\alpha} \\
8.4 \mathrm{G} \mathrm{HZ} / .4 \mathrm{G} \mathrm{HZ}\end{array}$ & $\begin{array}{c}\boldsymbol{\alpha} \\
4.85 \mathrm{G} \mathrm{HZ} 0.843 \mathrm{GHz}\end{array}$ \\
\hline IMG 1 J000 107+1914 & $\infty 018.53+191434.3$ & wVSs & 504.2 & 197 & & 265.1 & 221 & 3.1 & 024 & -0.14 & -0.36 & \\
\hline$[\mathrm{HB} 89] 0004+171$ & $\infty 0547.35+172815.4$ & wVSS & 95.4 & 159 & & 225.5 & 225 & 2.89 & 0.28 & 0.00 & 0.48 & \\
\hline IMG1 J000655+1416 & $\infty 0557.52+141546.9$ & nVSS & 79.6 & 121 & & 183.4 & 381 & 32 & 0.33 & 0.54 & 0.47 & \\
\hline PIS $0000+016$ & $\infty 0958.53+0155552$ & nvSs & 98.2 & 140 & & 270.7 & 325 & 2.95 & 0.53 & 0.14 & 0.57 & \\
\hline PrS $0012-312$ & $\infty 14.37 .96-305918.8$ & wVss & & 140 & 226.4 & 192.8 & & 2.79 & 0.26 & & & 0.27 \\
\hline [HE89] 0014+813 & $\infty 178.51+81358.3$ & HVss & 1303.8 & & & 692.5 & & 3.37 & & & -0.35 & \\
\hline 8766001510.94525527 & $\infty 1751.78+531219.4$ & nVSS & & 650 & & 393.3 & & 2.57 & -0.40 & & & \\
\hline Pis $0017-307$ & $\infty 1942.72-303119.9$ & nvss & & 649 & 5045 & 507 & 495 & 2.58 & -0.20 & -0.02 & & -0.14 \\
\hline $630020+4 t 0$ & $\infty 2241.33+44199.1$ & NVSS & & 40 & & 172.9 & 767 & 2.99 & 1.18 & 1.11 & & \\
\hline B R J0030-5129 & $\infty 03034.36-512946.2$ & sumus & & 76 & 70.4 & & & 4.17 & & & & -0.04 \\
\hline
\end{tabular}

Table 2. Sample table of 307 sources preliminary list. Position, obtained flux measurements, and calculated spectral indices.

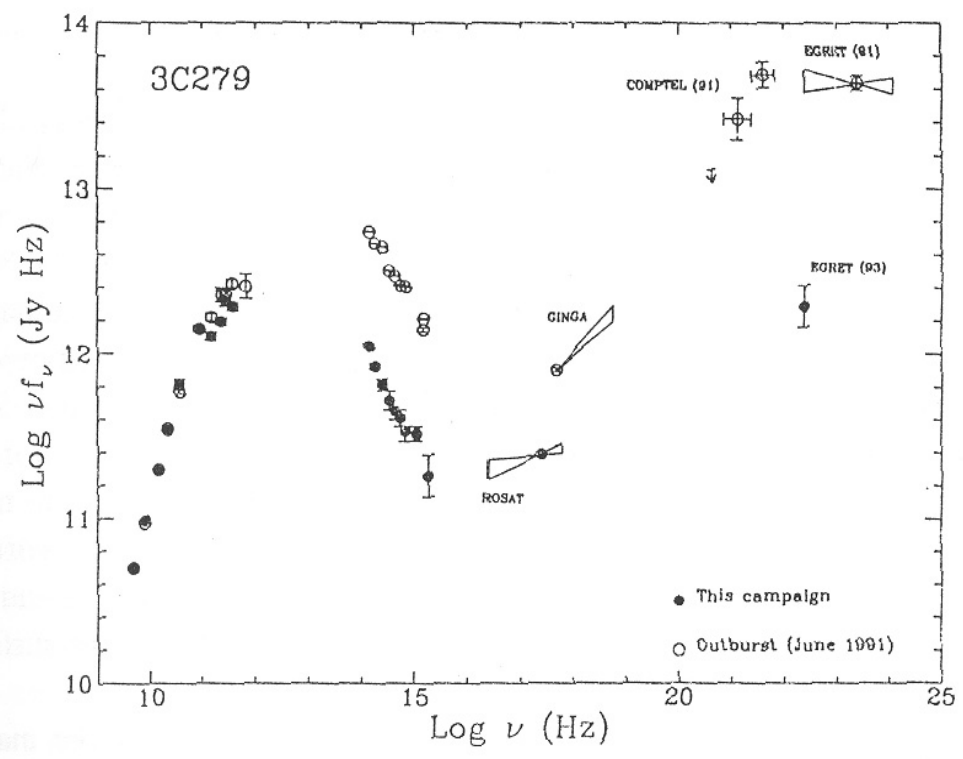

Figure 1. Spectral Energy Distribution of a well-studied blazar 3C279. Double peak signature can be seen from the data points taken at two observational campaigns. Maraschi et al. 1994. 


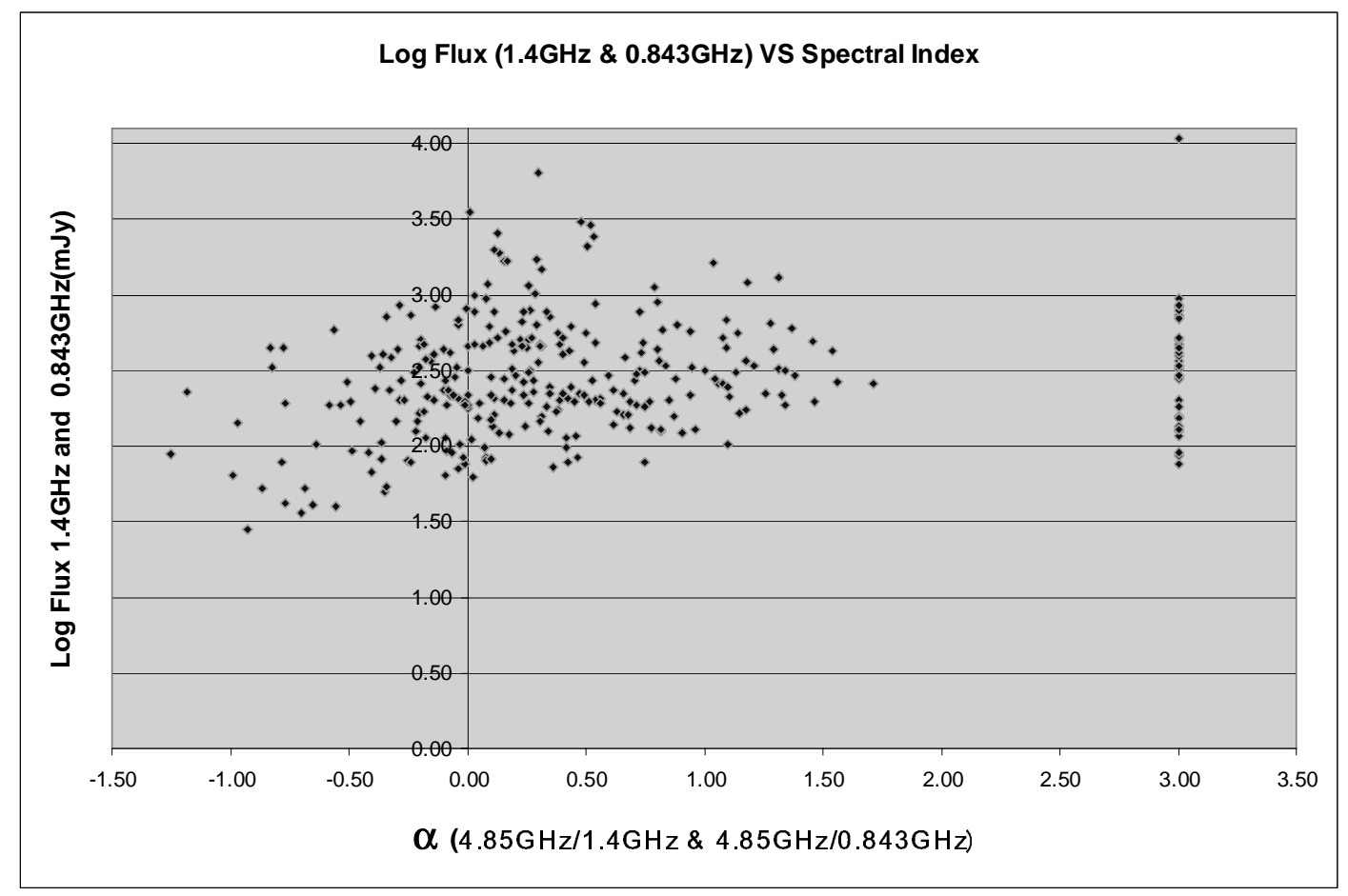

Figure 2. Flux at $1.4 \mathrm{GHz}$ and $0.843 \mathrm{GHz}$ vs. Spectral Index distribution calculated with flux ratio of 4.85GHz/1.4GHz and $4.85 \mathrm{GHz} / 0.843 \mathrm{GHz}$. A value of 3.0 was given to sources with not found spectral index

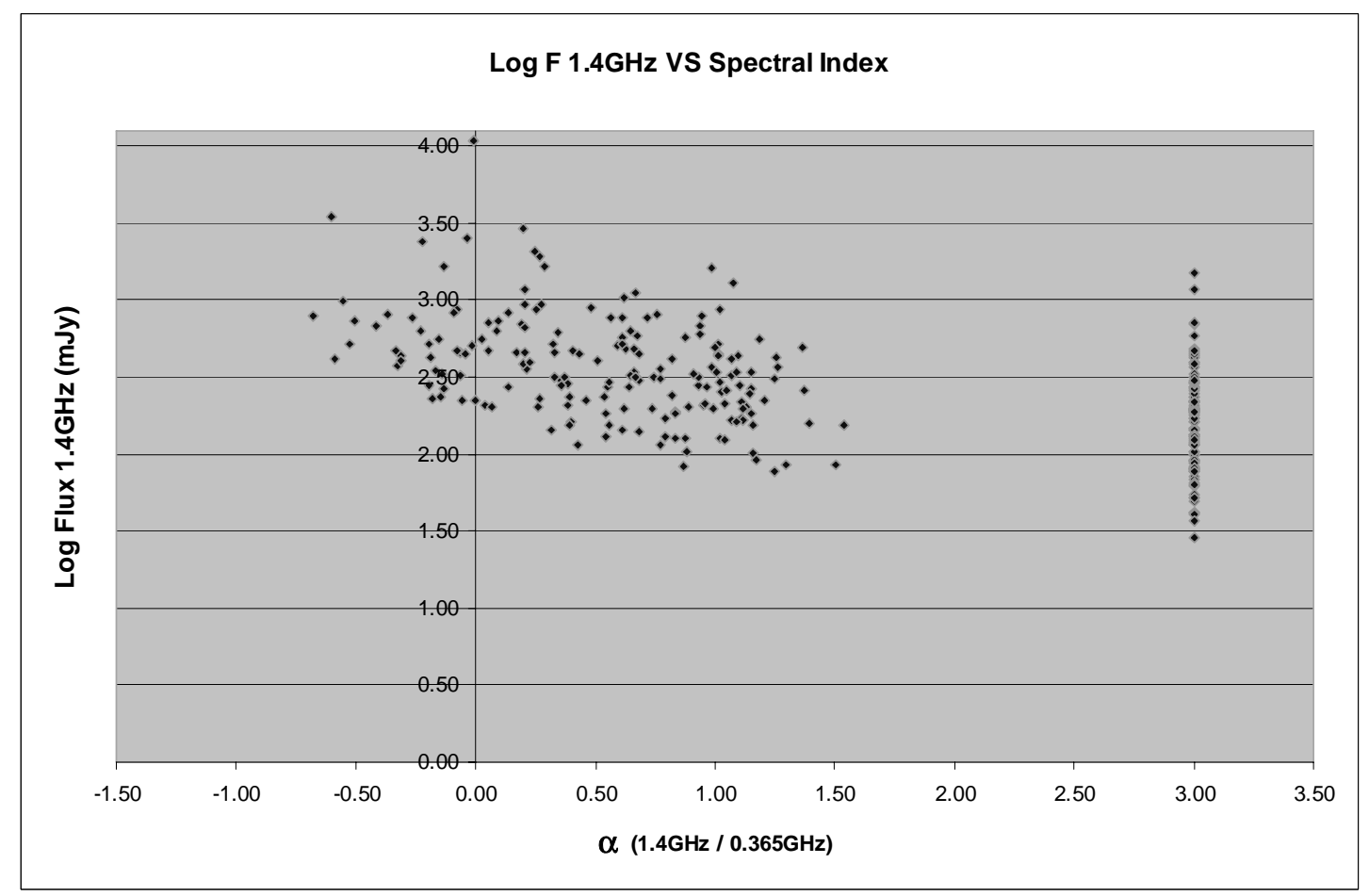

Figure 3 Flux at $1.4 \mathrm{GHz}$ vs. Spectral Index distribution calculated with flux ratio of $1.4 \mathrm{GHz} / 0.365 \mathrm{GHz}$ 


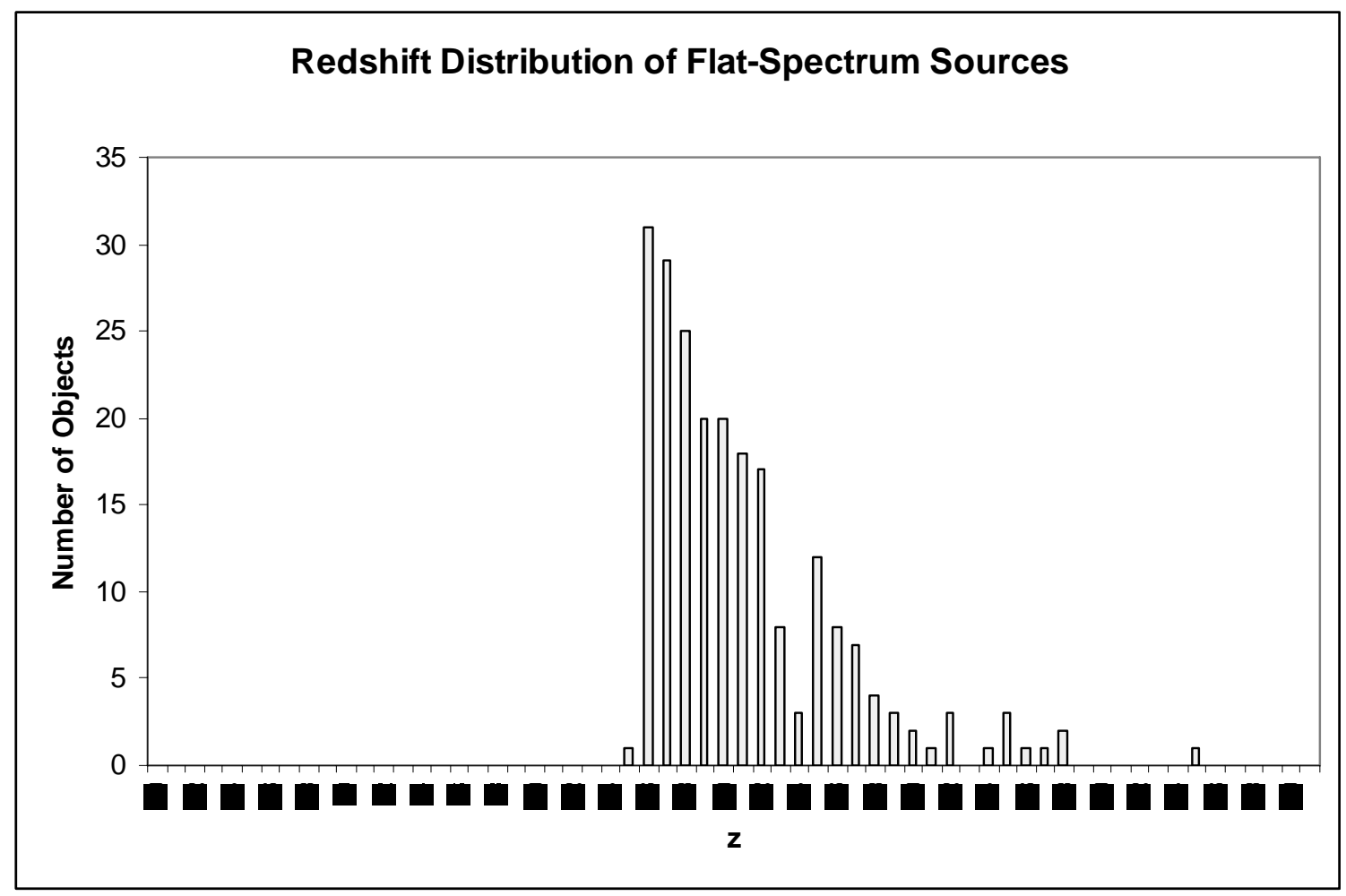

Figure 4. Redshift Distribution of all flat-radio spectrum sources 


\title{
Using Boosted Decision Trees to Separate
}

\section{Signal and Background in $B \rightarrow X_{s} \gamma$ Decays}

\author{
James Barber \\ Office of Science, SULI Program \\ University of Massachusetts, Amherst \\ Stanford Linear Accelerator Center \\ Stanford, California
}

August 23, 2006

Prepared in partial fulfillment of the requirements of the Office of Science, U.S. Department of Energy Science Undergraduate Laboratory Internship (SULI) Program under the direction of Philip Bechtle at the Stanford Linear Accelerator Center.

Participant:

Signature

Research Advisor:

Signature 


\section{TABLE OF CONTENTS}

Abstract

$\begin{array}{ll}\text { Introduction } & 1\end{array}$

$\begin{array}{lr}\text { Materials and Methods } & 5\end{array}$

$\begin{array}{lr}\text { Results } & 6\end{array}$

$\begin{array}{ll}\text { Discussion and Conclusions } & 7\end{array}$

$\begin{array}{lr}\text { Acknowledgments } & 8\end{array}$

$\begin{array}{lr}\text { References } & 8\end{array}$

$\begin{array}{ll}\text { Figures } & 10\end{array}$ 


\begin{abstract}
Using Boosted Decision Trees to Separate Signal and Background in $B \rightarrow X_{s} \gamma$ Decays. JAMES BARBER (University of Massachusetts, Amherst, Amherst, MA 01003) PHILIP BECHTLE (Stanford Linear Accelerator Center, Stanford, CA 94025)

The measurement of the branching fraction of the flavor changing neutral current $B \rightarrow$ $X_{s} \gamma$ transition can be used to expose physics outside the Standard Model. In order to make a precise measurement of this inclusive branching fraction, it is necessary to be able to effectively separate signal and background in the data. In order to achieve better separation, an algorithm based on Boosted Decision Trees (BDTs) is implemented. Using Monte Carlo simulated events, 'forests' of trees were trained and tested with different sets of parameters. This parameter space was studied with the goal of maximizing the figure of merit, Q, the measure of separation quality used in this analysis. It is found that the use of 1000 trees, with 100 values tested for each variable at each node, and 50 events required for a node to continue separating give the highest figure of merit, $\mathrm{Q}=18.37$.
\end{abstract}




\section{INTRODUCTION}

A rare $B$ meson decay explored at BaBar is the process $B \rightarrow X_{s} \gamma$, in which a $B$ meson decays to a photon and a hadronic final state containing an $s$ quark from the $b \rightarrow s \gamma$ transition. A Feynman diagram of this transition is given in Figure 1. This process is predicted to have a branching fraction of $\mathcal{B}\left(B \rightarrow X_{s} \gamma\right)=(3.61 \pm 0.49) \times 10^{-4}$ [1], which agrees well with the

world average of previous measurements of $\mathcal{B}\left(B \rightarrow X_{s} \gamma\right)=(3.55 \pm 0.26) \times 10^{-4}$ [2]. With a future improvement in the theoretical uncertainty expected, an improvement in the precision of the experimental measurement would increase the sensitivity for new physics.

The $B \rightarrow X_{s} \gamma$ measurement presented here is fully inclusive, meaning any final state is allowed. This has the advantage of reduced theoretical uncertainties compared to exclusive measurements. These uncertainties stem from the predictions of specific final states, such as $B \rightarrow K^{*} \gamma$, and are due to uncertainties in the fragmentation, i.e. the calculation of how the remaining quarks combine to form hadrons. These uncertainties are largely avoided by allowing for all possible hadronic states. Reduced information about the kinematics of the entire final state, however, makes background suppression difficult because neither of the two $B$ mesons in the event are reconstructed.

The background is split into two categories: $B \bar{B}$ and continuum. $B \bar{B}$ background refers to decays from $B \bar{B}$ events not of the type $B \rightarrow X_{s} \gamma$. Continuum background, by contrast, is comprised of all non- $B \bar{B}$ events and is present at and below energies of $B \bar{B}$ events. To gain information about the continuum background, $10 \%$ of the data is taken at energies too low for $B \bar{B}$ production. Thus all data taken at 'off-peak' energies is continuum, while 'on-peak' data is a mix of continuum and $B \bar{B}$ backgrounds. Expected amounts of background and signal data are shown in Figure 2a, where it can be seen that the amount of background must be reduced by 3 orders of magnitude in order to detect a significant signal. Figure $2 \mathrm{~b}$ shows the result of a Fisher discriminant method of separation using event-shape variables and lepton tagging to separate signal and background. Lepton tagging is a method of identifying 
$B \bar{B}$ events by requiring the presence of a high momentum lepton (from the decay $B \rightarrow X l \nu$ ) in order to accept an event. The higher the momentum of the lepton is required to be, the less likely it is to have come from a continuum event. The branching fraction of a semileptonic $B$ decay is, however, only about $10 \%$, so in using this method at least $90 \%$ of $B \bar{B}$ events are rejected. The Fisher discriminant method of signal and background separation, used after lepton tagging, successfully eliminated $99 \%$ of the background but also rejected $95 \%$ of the signal. To reduce the amount of signal rejected, more advanced techniques for the suppression of both the continuum and $B \bar{B}$ backgrounds are needed. With these advanced techniques it is possible to use both the event-shape and lepton tagging variables concurrently to increase the selection efficiency.

The current technique used in the extraction of signal from background data is based on an Artificial Neural Network (ANN). When variables with low individual separation power and non-negligible, non-linear correlations (such as the event-shape variables used in this analysis) are used to separate signal and background, ANNs outperform methods based on a likelihood estimator or Fisher discriminant. In order to obtain high separation from variables with low separation power, an ANN must be 'trained' on Monte Carlo (MC) simulated data. By varying the importance of different variables (via adjusting the variables' weights) and combining variables in different manners, an ANN can learn which of these variable combinations and weight values yield the highest ratio of signal to background. The variable combination and weight adjustment is done in hidden layers of the ANN (as shown in Figure 3), the exact process of which is completely concealed from the user. This learning process, of re-weighting and re-combining variables in order to achieve the best signal and background separation, is referred to as 'training'.

One of the drawbacks in using an ANN is that it is possible to decrease the total separation power by giving the ANN too many variables with low separation power. Another drawback of an ANN is that ANNs are very sensitive to their training; the order of input variables in the training may affect the output, and if the Monte Carlo sample used to train the ANN 
does not very closely model the real data, the separation power of an ANN can suffer more than that of other methods.

In order to avoid these problems, an algorithm implementing Boosted Decision Trees (BDTs) has been developed to perform the signal and background separation. This method, like an ANN, must be trained to yield the best separation of signal and background (see [3] for a more detailed explanation of this process). To train a BDT, a number of MC events are chosen as training events and put into a 'root node'. The algorithm then iterates through each variable, finding the value at which a selection would give the highest signal and background separation. The variable that would give the highest separation is chosen and the training events are subjected to this selection. Events are sent to the left or right child, depending on whether the selection classifies them as signal or background. At each new node a variable and value are chosen from which a selection is made. The events are again separated and the process is repeated. Nodes are separated until they contain less than some minimum number of events or have a signal to background (or background to signal) ratio greater than a given limit. Nodes are then classified as either 'signal' or 'background' depending on whether the majority of events in that node is signal or background, respectively (see Figure 4). Misclassified signal and background events (e.g. signal events in background nodes or vice versa) are given an increased weight and the entire process is started again, with a new root node established and a new tree created. The result of increasing the weights of these previously misclassified events is that these events become more important when determining signal to background separation in the next iteration. In this way, a specified number of trees is created and the training is complete.

After training, the resulting 'forest' of trees must be tested to determine how well it separates signal and background. Events chosen for testing (of which none were used for training) are sent through each tree in the forest, and for each event a likelihood value is calculated. This value is equal to the number of times an event ends in a signal node divided by the number of trees it is sent through. An event classified as signal by every tree would 
thus have a likelihood value of 1 , whereas an event always classified as background would have a likelihood value of 0 . In this way, signal events tend to bunch closer to 1 than 0 , while background events tend toward 0. A threshold value is determined, which is equal to the likelihood value about which a selection made would give the best signal and background separation.

In order to determine superiority of one method over another, we must have some way to compare the quality of separation. We calculate a figure of merit, Q, for each separation method:

$$
Q=\frac{S}{\sqrt{S+B}}
$$

where $S$ is the number of signal events correctly classified as signal and $B$ is the number of background events incorrectly classified as signal. Because we are dealing with two types of background $-B \bar{B}$ and continuum $-B$ is defined as

$$
B=N_{B}\left(1+f \cdot B_{M C e r r o r}\right)+\frac{N_{C}}{1-f}
$$

where $N_{B}$ is the number of $B \bar{B}$ background events and $N_{C}$ is the number of continuum background events. The factor $f$ is the on-peak fraction, i.e. the fraction of data taken at the energy required for $B \bar{B}$ pair production. In typical BaBar data taking, $f$ is about $90 \%$. Hence, $(1-f)$ is the fraction of available off-peak data from which continuum measurements are made. The term $B_{\text {MCerror }}$ accounts for systematic uncertainties in the MC generated $B \bar{B}$ background events.

Once the forest of trees with the highest figure of merit has been formed, it is ready to be used with real data. Each data event is sent through the forest of trees and, just like with testing events, a likelihood value is determined. If the likelihood value is above the previously determined threshold value, the event is classified as signal. 


\section{MATERIALS AND METHODS}

Rather than creating a new implementation of the BDT algorithm, modifications were made to an existing implementation contained in the Toolkit for Multivariate Analysis (TMVA) [4]. A program was created to interface with the TMVA package, allowing us to input data. As mentioned above, BDTs must be 'trained' to separate signal and background and then tested to measure their efficiencies. Because of this, parameters indicating the number of events to use for training need to be passed into the program along with the data used in the training and testing. It is important for the BDT to be trained on a sample of data representative of the entire data range so that variations in the data will be accounted for by the BDT. It is also important that the testing sample be statistically independent of the training sample and taken from the same data range to ensure proper measurement of separation efficiency. In this way, the agreement of the results of the training and testing can be used to ensure that the BDT does not classify the data according to particular features of the training sample (known as "over-training") but separates based on general event properties of the signal and background.

It was found that the method used by the TMVA to select training and testing events was not appropriate for our set of input events, because the events chosen were not representative of the entire event range. Let us take, for instance, a sample of 100,000 $\left(N_{\text {total }}\right)$ events, with $10,000\left(N_{\text {train }}\right)$ used for training and 50,000 $\left(N_{\text {test }}\right)$ used for testing. The TMVA would use events numbered 1-10,000 for training and events numbered 10,001-60,000 for testing. If different experimental setups were used to collect data, and different MC data samples were generated to reflect those changes, testing could be done on a continuous subset of events representative of only a portion of the actual data. In this case, the calculated quality of separation would be inaccurate. It is then obvious that training and testing event samples must be selected from throughout the entire data set to protect against training and testing on events from different experimental setups. To fix this problem, changes were made to the 
TMVA code to ensure that events used for training and testing are taken from the entire data range. To do this, we required that if $N_{\text {train }}$ events were asked for, approximately one out of every $\frac{N_{\text {total }}}{N_{\text {train }}}$ events is selected for training.

A second issue in the selection of events that needed to be addressed concerned the ratio of signal to background events. If the ratio of signal to background events in our Monte Carlo data sample is $\frac{N_{s i g}}{N_{b k g}}$, it is imperative that our training sample has this same ratio of signal to background. As implemented, the TMVA used equal numbers of signal and background events. Failure to retain the proper signal to background ratio would cause incorrect values of the figure of merit to be calculated, thus giving an incorrect assessment of the separation power of the algorithm. The algorithm used to select events was again changed to accommodate the needs of our analysis.

With the aforementioned problems fixed, we began training and testing forests of trees using different parameter values in order to find the setup yielding the maximal figure of merit. From a data set of $749,684 \mathrm{MC}$ events, 100,000 events were chosen for training. The number of trees in the forest $\left(N_{\text {trees }}\right)$ was either 500 or 1,000; the minimum number of events $\left(N_{\text {minEvents }}\right)$ required for a node to be separated further was either 50 or 100; and the number of values checked for each variable in determining how to best separate a node $\left(N_{\text {cuts }}\right)$ was either 25,50 , or 100 . At the conclusion of testing, figures of merit were calculated for each parameter setup so that the separation quality of each might be compared.

\section{RESULTS}

Table 1 shows the different parameter setups for which trees were trained and tested, as well as the resultant figure of merit for each setup. As can be seen, parameter set ' $b$ ' is found to give the highest figure of merit. The results of the testing conducted with this parameter set are shown graphically in Figure 5, where the likelihood value is plotted on the x-axis in red for signal, blue for $B \bar{B}$ background, and cyan for continuum background. The vertical line 
superimposed on the graph shows the threshold value at which a selection would yield the maximal figure of merit. This threshold value was found to be .595, which gave a figure of merit, Q, of 18.37. Plots representative of the selection quality are shown in Figures 6 and 7. Figure 6 shows the total number of signal and background events both before and after the selection algorithm. Figure 7 shows the efficiency of selection for the $B \rightarrow X_{s} \gamma$ signal as well as both the $B \bar{B}$ and continuum backgrounds. Note that a low efficiency is desirable for the backgrounds, as the efficiency is a measure of the number of events selected as signal.

\section{DISCUSSION AND CONCLUSIONS}

Table 1 suggests that the figure of merit increases as the number of trees $\left(N_{\text {trees }}\right)$ is increased, and also as the number of values (for each variable) used to determine where to best make a selection $\left(N_{\text {cuts }}\right)$ is increased. The results also indicate an increase in the figure of merit as the minimum number of events required in a node $\left(N_{\text {minEvents }}\right)$ is decreased, however this effect is small compared to the effect due to an increase in either $N_{\text {trees }}$ or $N_{\text {cuts }}$. The gain resulting from an increase in these two variables, however, comes at the cost of speed. The number of trees selected should scale linearly (as a roughly constant amount of computing must be done to train a tree), however increasing the value of $N_{\text {cuts }}$ greatly increases the time it takes to train each tree. This increase in computational time for each tree is so great when compounded over 1000 trees that it took 5 times longer to run the program with parameter setup 'b' than with setup 'a'.

To determine whether this BDT algorithm is preferable to the ANN currently used by the analysis requires the generation of comparable figures of merit for each method. This can be realized by designing within a Monte Carlo event sample a group of events designated for training and another set of events designated for testing. In running the BDT and ANN on this same set of events and calculating figures of merit for each method, a direct comparison can be made. 
It is hoped that the result of such a comparison will show the BDT implementation worked on this summer superior to the ANN currently in use, and that the use of BDTs in separating signal and background will lead to increased precision in the analysis of the $B \rightarrow X_{s} \gamma$ decay. This precision will help the search for physics beyond the standard model as it puts tighter limits on the experimental value for this branching fraction. If it is found that the theoretical predictions and experimental measurements for this branching fraction do not agree it may point to the presence of an until now undetected massive particle, such as a Higgs or SUSY particle, in the radiative penguin loop.

\section{ACKNOWLEDGMENTS}

I would sincerely like to thank my mentor Philip Bechtle for his guidance throughout this

project and his eagerness to discuss all aspects of particle physics, not merely those pertaining to my research. I would also like to thank Rainer Bartoldus for his thoughts and suggestions on how to best present my work. I would like to acknowledge the U.S. Department of Energy, Office of Science and all those at the Stanford Linear Accelerator Center for giving me the opportunity to participate in the SULI program. Thanks to everyone involved, it has truly been a rewarding and educational experience.

\section{REFERENCES}

[1] Heavy Flavor Averaging Group (HFAG) and E. Barberio, et al., "Averages of b-hadron properties at the end of 2005," 2006. http://www.slac.stanford.edu/xorg/hfag/

[2] T. Hurth, E. Lunghi and W. Porod, "Untagged $B \rightarrow X_{s d} \gamma \mathrm{CP}$ asymmetry as a probe for new physics," Nucl. Phys., vol. B704, pp. 56-74, 2005.

[3] B. P. Roe, et al., "Boosted decision trees, an alternative to artificial neural networks," Nucl. Instrum. Meth. vol. A543, pp. 577-584, 2005. 
[4] TMVA package developed by Andreas Höcker (CERN), Jörg Stelzer (CERN), Helge Voss (MPI-KP Heidelberg), Kai Voss (U. of Victoria), and Xavier Prudent (LAPP-Annecy). http://tmva.sourceforge.net/ 


\section{FIGURES}

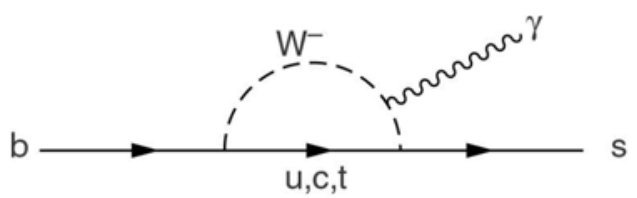

Figure 1: Feynman diagram of $b \rightarrow s \gamma$ transition.

\begin{tabular}{|ll|ll|}
\hline Parameter setup a & & Parameter setup b & \\
$N_{\text {trees }}$ & 1000 & $N_{\text {trees }}$ & 1000 \\
$N_{\text {minEvents }}$ & 50 & $N_{\text {minEvents }}$ & 50 \\
$N_{\text {cuts }}$ & 50 & $N_{\text {cuts }}$ & 100 \\
figure of merit: & 18.21 & figure of merit: & 18.37 \\
\hline Parameter setup c & & Parameter setup d & \\
$N_{\text {trees }}$ & 500 & $N_{\text {trees }}$ & 1000 \\
$N_{\text {minEvents }}$ & 50 & $N_{\text {minEvents }}$ & 100 \\
$N_{\text {cuts }}$ & 50 & $N_{\text {cuts }}$ & 50 \\
figure of merit: & 18.04 & figure of merit: & 18.18 \\
\hline Parameter setup e & & \\
$N_{\text {trees }}$ & 500 & & \\
$N_{\text {minEvents }}$ & 50 & & \\
$N_{\text {cuts }}$ & 25 & & \\
figure of merit: & 17.91 & & \\
\hline
\end{tabular}

Table 1: Parameter setups and corresponding figures of merit 


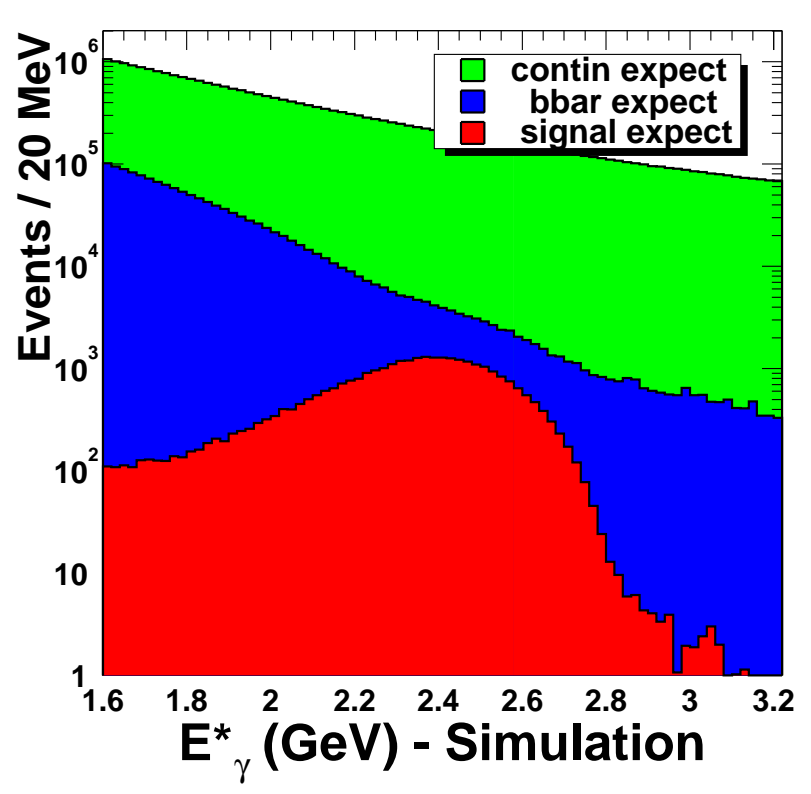

(a)

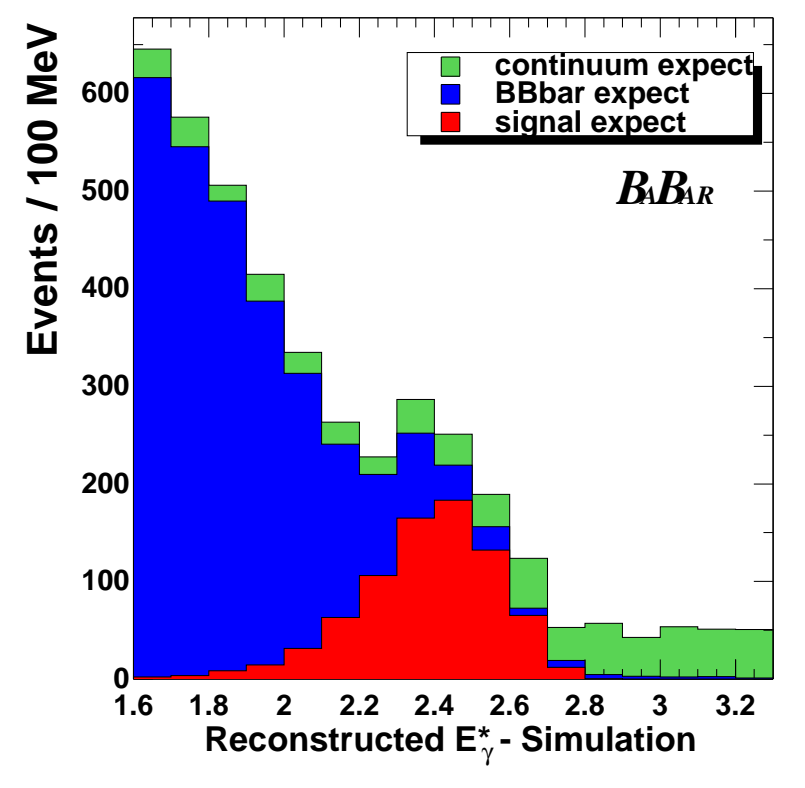

(b)

Figure 2: Signal and background plotted before (a) and after (b) selection including the Fisher method of separation.

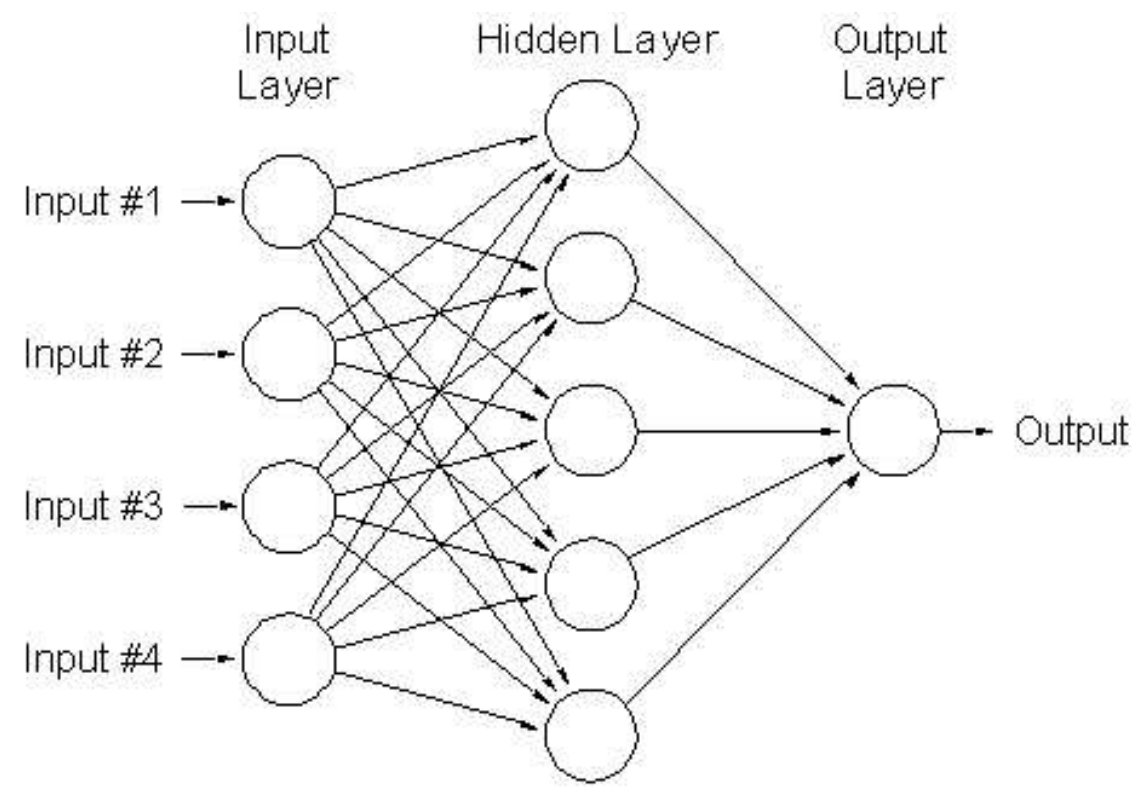

Figure 3: Variable weighting and combination are done in the hidden layers of an ANN 


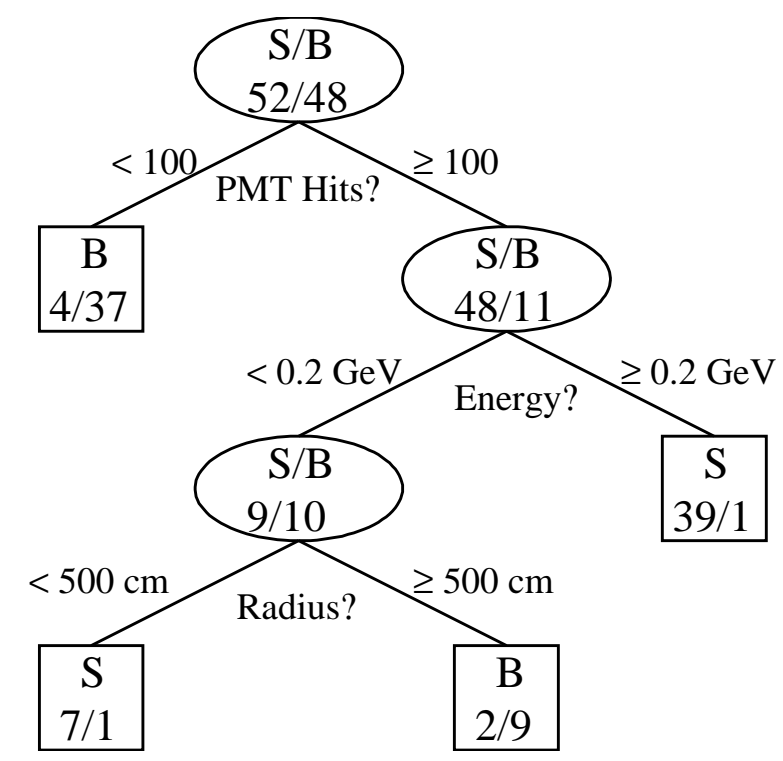

Figure 4: Example splitting of a BDT

\section{Signal and Background}

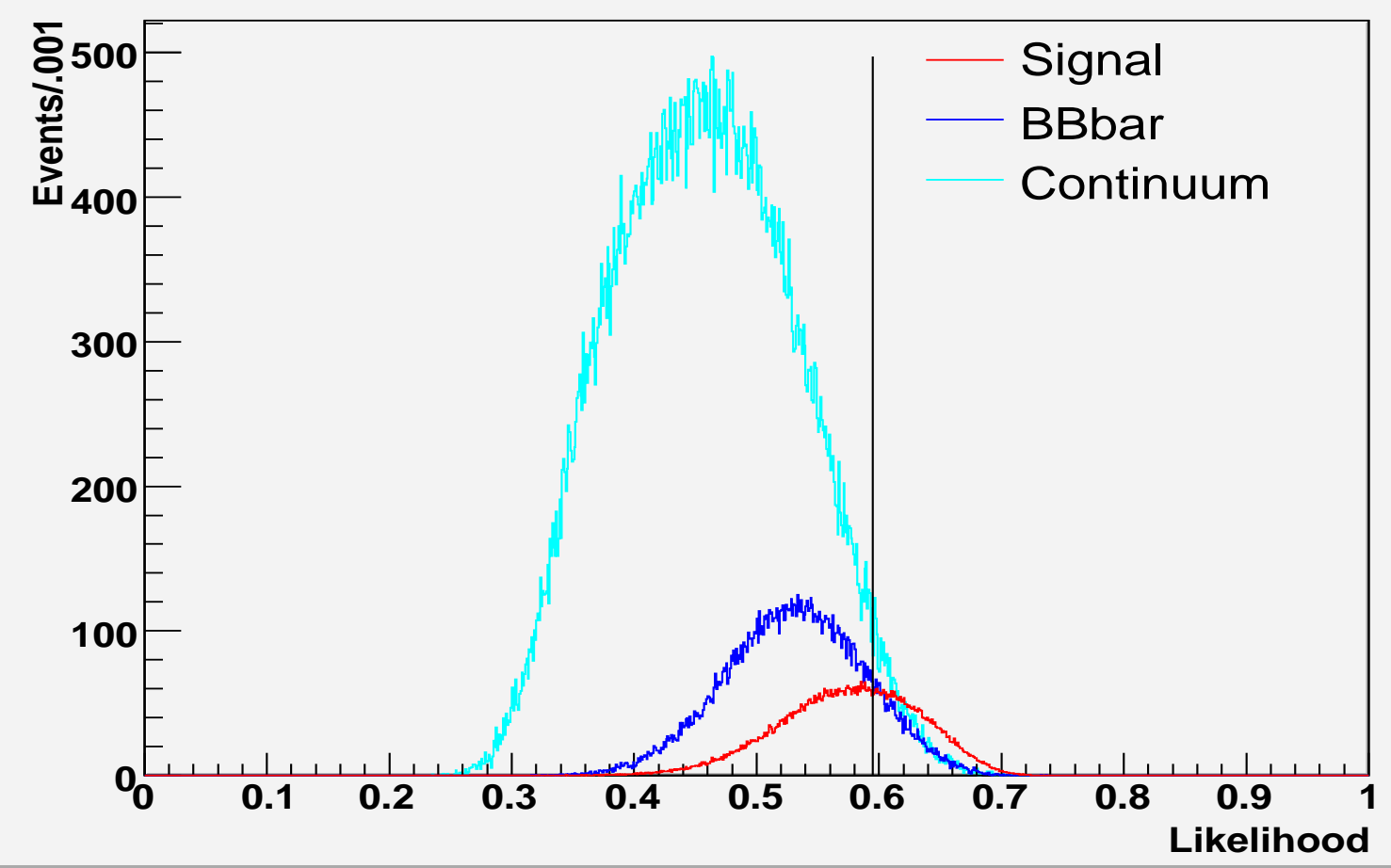

Figure 5: Likelihood values for Monte Carlo testing events, using parameter set 'b'. A selection at .595 (indicated by verticle line) gives a figure of merit of 18.37 . 


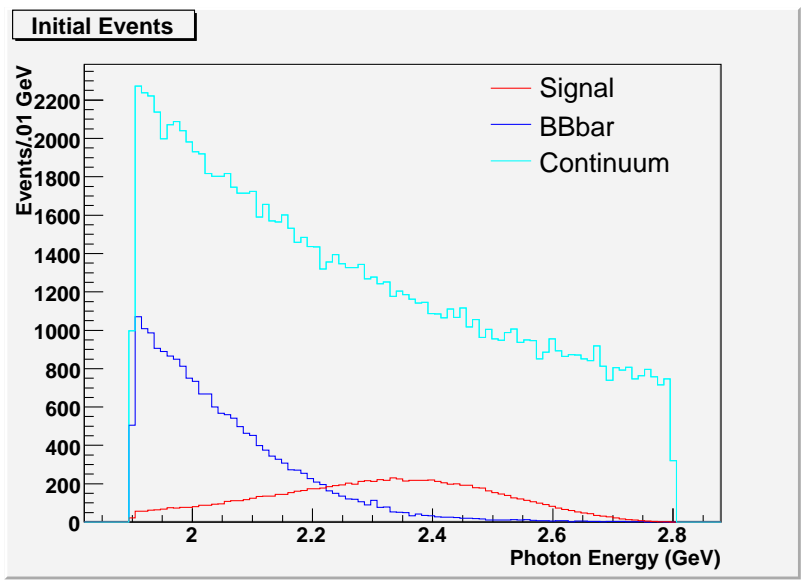

(a)

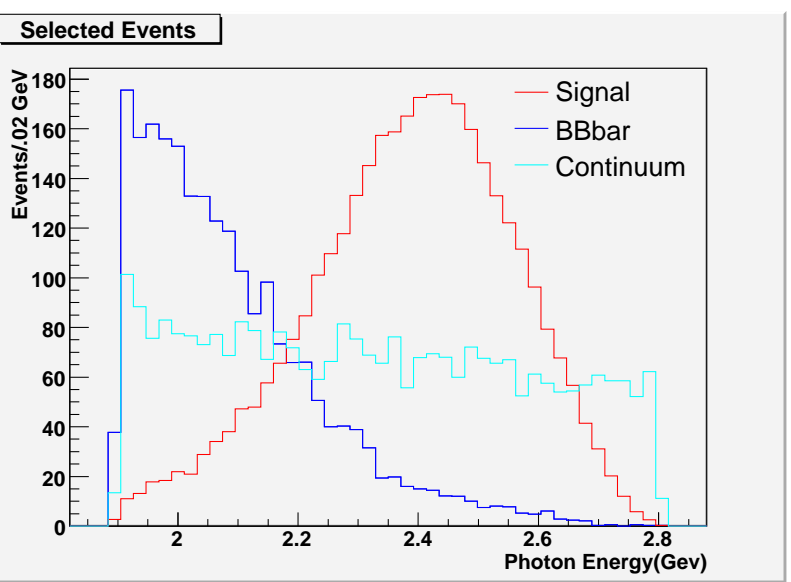

(b)

Figure 6: Number of signal and background events before (a) and after (b) BDT separation with parameter set 'b'.

\section{Efficiencies}

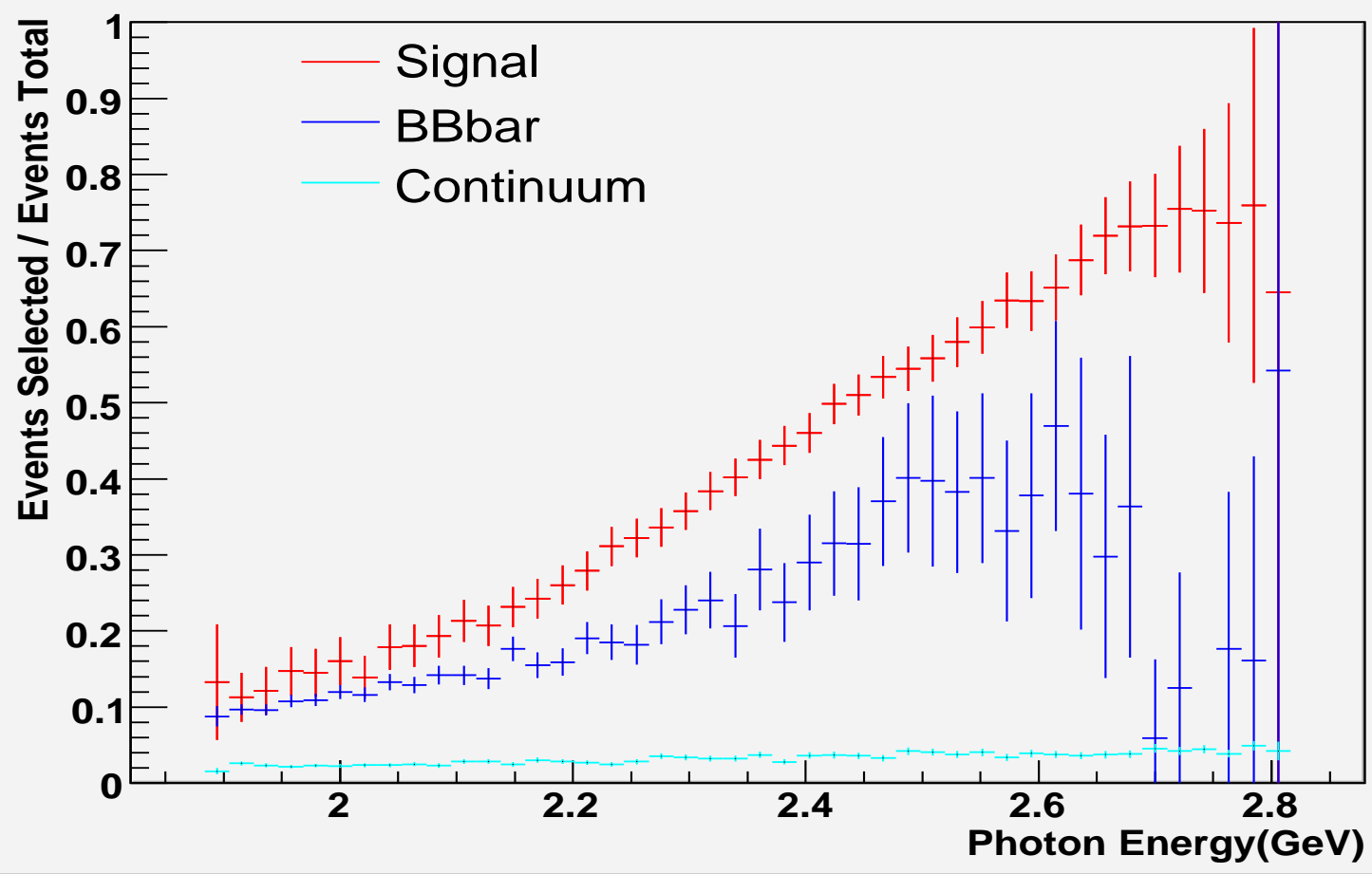

Figure 7: Signal and background efficiencies of BDT algorithm with parameter set 'b'. 


\title{
Improvement of PEP-II Linear Optics with a MIA-Derived Virtual Accelerator
}

\author{
Ben Cerio \\ Office of Science, Science Undergraduate Laboratory Internship (SULI) \\ Colgate University \\ Stanford Linear Accelerator Center \\ Stanford, California
}

August 18, 2006

Prepared in partial fulfillment of the requirements of the Office of Science, Department of Energy's Science Undergraduate Laboratory Internship under the direction of Yiton Yan in the Beam Physics Department at the Stanford Linear Accelerator Center.

\section{Participant:}

Research Advisor: 


\section{Table of Contents}

Abstract........................................................... iii.

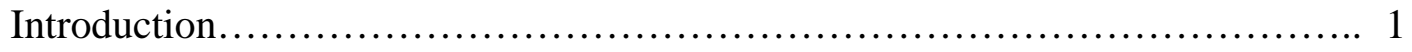

Materials and Methods.................................................... 2

Results............................................................ 7

Discussion and Conclusion............................................... 9

Acknowledgements................................................. 10

References........................................................ 10

Figures........................................................... 11 


\begin{abstract}
Improvement of PEP-II Linear Optics with a MIA-Derived Virtual Accelerator. BEN CERIO (Colgate University, Hamilton, NY 13346, USA) YITON YAN (Stanford Linear Accelerator Center, Stanford, CA 94309, USA).
\end{abstract}

In several past studies, model independent analysis, in conjunction with a virtual accelerator model, has been successful in improving PEP-II linear geometric optics. In many cases, optics improvement yielded an increase in machine luminosity. In this study, an updated characterization of linear optics is presented. With the PEP-II beam position monitor (BPM) system, four independent beam centroid orbits were extracted and used to determine phase advances and linear Green’s functions among BPM locations. A magnetic lattice model was then constructed with a singular value decompositionenhanced least-square fitting of phase advances and Green’s functions, which are functions of quadrupole strengths, sextupole feed-downs, as well as BPM errors, to the corresponding measured quantities. The fitting process yielded a machine model that matched the measured linear optics of the real machine and was therefore deemed the virtual accelerator. High beta beat, as well as linear coupling, was observed in both LER and HER of the virtual accelerator. Since there was higher beta beating in LER, focus was shifted to the improvement of this ring. By adjusting select quadrupoles of the virtual LER and fitting the resulting beta functions and phase advances to those of the desired lattice, the average beta beat of the virtual machine was effectively reduced. The new magnet configuration was dialed into LER on August 10, 2006, and beta beat was reduced by a factor of three. After fine tuning HER to match the improved LER for optimal collision, a record peak luminosity of $12.069 \times 10^{33} \mathrm{~cm}^{-2} \mathrm{~s}^{-1}$ was attained on August 16, 2006. 


\section{INTRODUCTION}

PEP-II is an electron-positron collider with asymmetric energy storage rings: the high energy ring (HER) which stores electrons at $9.0 \mathrm{GeV}$, and the low energy ring (LER) which stores positrons at $3.1 \mathrm{GeV}$. These two rings intersect at the interaction point (IP), where the BaBar detector is located. PEP-II, a complex and dynamic machine, must be regularly upgraded to remain at the frontier of particle physics research.

The improvement of linear optics in PEP-II has, in the past, resulted in greater luminosity. After taking BPM measurements, it is possible to extract 4 independent beam centroid orbits. These 4 orbits are then inputted into a model independent analysis MIA for extracting critical linear optics parameters [1,2]. With the ideal lattice as the initial model, the parameters of the model are fitted with a singular value decomposition (SVD)enhanced least square fitting technique to the corresponding measurement-derived quantities, yielding a computer virtual machine that matches the linear optics of PEP-II LER or HER. The strengths of select magnetic quadrupoles of the virtual machine are varied and a new magnet configuration that minimizes beta beating and linear coupling is determined. The improved magnet configuration may then be applied to PEP-II, thus improving the linear optics and perhaps the luminosity.

For the past several months, PEP-II Operations was pushing current as a strategy

to increase luminosity. Although this strategy was successful in breaking peak luminosity records, we saw an increase in beta beat in LER and HER. This degradation of linear optics called for an updated MIA-derived virtual accelerator from which we could determine a beta-beat-mitigating solution. 
In this study, we continued to use MIA as a tool for optimization of PEP-II optics. We discuss the process of constructing a virtual machine derived from BPM measurements. We then present a comparison of the virtual and ideal lattice optics, with a focus on betatron amplitude and phase, linear coupling, and IP optics characterization. We conclude with a discussion of the creation of an improved virtual LER that minimizes beta beat and the subsequent improvement of the real machine.

\section{MATERIALS AND METHODS}

\section{BPM DATA ACQUISITION}

In PEP-II HER there are 38 single view horizontal (x), 40 single view vertical (y), and 215 double view BPMs, corresponding to a maximum of $253 \mathrm{x}$ data and $255 \mathrm{y}$ data. The LER has 46 single view horizontal, 44 single view vertical, and 229 double view BPMs. PEP-II BPMs are capable of acquiring turn-by-turn data at a resolution of a few microns, allowing for precise orbit measurements. With a single bunch in HER (LER), we resonantly excited the beam with the transverse feedback kicker in straight 4 at the horizontal betatron tune for 1024 turns, resulting in a 1024-by-253 (275) matrix of x data and a 1024-by-255 (273) matrix of y data. We also excited the beam vertically at the vertical betatron tune and stored another set of $\mathrm{x}$ and y BPM buffer data.

\section{EXTRACTION OF ORBITS}

We performed a tune-matched fast Fourier transform on the columns of the raw BPM buffer data, which allowed us to extract the resonant betatron mode. The resonant mode contains two degrees of freedom, an imaginary part and a real part, which represent the sine-like orbit and cosine-like orbit, respectively. Since this was done for transverse 
directions ( $\mathrm{x}$ and $\mathrm{y}$ ) and for both beam excitations (horizontal and vertical), we obtained a four orbit matrix Z at each BPM location. For an arbitrary location a,

$$
Z^{a}=\left[\begin{array}{llll}
\vec{Z}_{1}^{a} & \vec{z}_{2}^{a} & \vec{Z}_{3}^{a} & \vec{Z}_{4}^{a}
\end{array}\right]=\left[\begin{array}{cccc}
x_{1}^{a} & x_{2}^{a} & x_{3}^{a} & x_{4}^{a} \\
x_{1}^{\prime a} & x_{2}^{\prime a} & x_{3}^{\prime a} & x_{4}^{\prime a} \\
y_{1}^{a} & y_{2}^{a} & y_{3}^{a} & y_{4}^{a} \\
y_{1}^{\prime a} & y_{2}^{\prime a} & y_{3}^{\prime a} & y_{4}^{\prime a}
\end{array}\right]
$$

where $\vec{z}_{1}^{a}$ and $\vec{z}_{2}^{a}$ are orbits from horizontal excitation, $\vec{z}_{3}^{a}$ and $\vec{z}_{4}^{a}$ are orbits from vertical excitation, and $x^{\prime}$ and $y^{\prime}$ have not been measured. Figure 1 shows four independent beam orbits. Note the coupling between the $\mathrm{x}$ and y orbits near IP (BPM 155-156). PHASE ADVANCES AND LINEAR GREEN'S FUNCTIONS

The independent orbits derived from measurement allowed us to calculate betatron phase advances and linear Green's functions from which we determined critical linear optics parameters. To calculate the phase $\mu$ at an arbitrary location a, we have

$$
\mu_{a}=\tan ^{-1}\left[\frac{\operatorname{Im}\left(x^{a}\right)}{\operatorname{Re}\left(x^{a}\right)}\right],
$$

where $x_{a}$ is the Fourier-transformed orbit at the betatron resonance. Phase advances at adjacent locations were simply calculated by subtraction [3]. This calculation assumed no BPM errors. BPM gains were canceled in the ratio; however, cross coupling remained and phase advances were therefore corrected after fitting of the virtual model for which BPM cross coupling was a fitting variable.

MIA also determined linear Green’s functions to which we fit the corresponding model Green’s functions. For arbitrary lattice locations a and b, we defined a linear map $Z^{b}=R^{a b} Z^{a}$, where $R^{a b}$ is the transfer matrix from a to b, and $Z^{a}$ and $Z^{b}$ are the 
orbit matrices at these locations. Since transfer matrices are canonical transformations, we applied the symplectic condition, $R^{a b T} S R^{a b}=S$, where

$$
S=\left[\begin{array}{cc}
S_{2 \times 2} & 0 \\
0 & S_{2 \times 2}
\end{array}\right] \text { with } S_{2 \times 2}=\left[\begin{array}{cc}
0 & 1 \\
-1 & 0
\end{array}\right] \text {. }
$$

Using this condition, we defined an invariant matrix Q, where

$$
Q=Z^{b T} S Z^{b}=\left(R^{a b} Z^{a}\right)^{T} S R^{a b} Z^{a}=Z^{a T} S Z^{a} .
$$

The Green’s functions are merely transfer matrix elements and can be expressed as follows [5]:

$$
\begin{aligned}
& \left(x_{1}^{a} x_{2}^{b}-x_{2}^{a} x_{1}^{b}\right) / Q_{12}+\left(x_{3}^{a} x_{4}^{b}-x_{4}^{a} x_{3}^{b}\right) / Q_{34}=R_{12}^{a b}, \\
& \left(x_{1}^{a} y_{2}^{b}-x_{2}^{a} y_{1}^{b}\right) / Q_{12}+\left(x_{3}^{a} y_{4}^{b}-x_{4}^{a} y_{3}^{b}\right) / Q_{34}=R_{32}^{a b}, \\
& \left(y_{1}^{a} x_{2}^{b}-y_{2}^{a} x_{1}^{b}\right) / Q_{12}+\left(y_{3}^{a} x_{4}^{b}-y_{4}^{a} x_{3}^{b}\right) / Q_{34}=R_{14}^{a b}, \\
& \left(y_{1}^{a} y_{2}^{b}-y_{2}^{a} y_{1}^{b}\right) / Q_{12}+\left(y_{3}^{a} y_{4}^{b}-y_{4}^{a} y_{3}^{b}\right) / Q_{34}=R_{34}^{a b} .
\end{aligned}
$$

The above expressions, however, assume that the BPM measurements are perfect. In fact, when we fitted our model to the measurement-derived Green's functions, we had to account for BPM gains and cross-plane coupling:

$$
\begin{aligned}
& R_{12}^{a b}=g_{x}^{b} \Re_{12}^{a b} g_{x}^{a}+g_{x}^{b} \Re_{14}^{a b} \theta_{x y}^{a}+\theta_{x y}^{b} \Re_{32}^{a b} g_{x}^{a}+\theta_{x y}^{b} \Re_{34}^{a b} \theta_{x y}^{a}, \\
& R_{32}^{a b}=g_{y}^{b} \Re_{32}^{a b} g_{x}^{a}+g_{y}^{b} \Re_{34}^{a b} \theta_{x y}^{a}+\theta_{y x}^{b} \Re_{12}^{a b} g_{x}^{a}+\theta_{y x}^{b} \Re_{14}^{a b} \theta_{x y}^{a}, \\
& R_{14}^{a b}=g_{x}^{b} \Re_{14}^{a b} g_{y}^{a}+g_{x}^{b} \Re_{12}^{a b} \theta_{y x}^{a}+\theta_{x y}^{b} \Re_{34}^{a b} g_{y}^{a}+\theta_{x y}^{b} \Re_{32}^{a b} \theta_{y x}^{a}, \\
& R_{34}^{a b}=g_{y}^{b} \Re_{34}^{a b} g_{y}^{a}+g_{y}^{b} \mathfrak{R}_{32}^{a b} \theta_{y x}^{a}+\theta_{y x}^{b} \Re_{14}^{a b} g_{y}^{a}+\theta_{y x}^{b} \Re_{12}^{a b} \theta_{y x}^{a},
\end{aligned}
$$

where $g_{x}$ and $g_{y}$ are BPM gains, and $\theta_{x y}$ and $\theta_{y x}$ are BPM cross coupling multipliers [5].

$R$ ' $s$ are the linear Green's functions in the measurement frame, while $\mathfrak{R}$ 's are the corresponding quantities of the model that are corrected accordingly. 


\section{SVD-ENHANCED LEAST-SQUARE FITTING}

After determining phase advances and linear Green's functions with MIA, we were in a position to construct a machine model by fitting model parameters to these phase advances and linear Green’s functions derived from measurement. We organized quantities into vectors and defined the fundamental fitting relationship $\vec{Y}(\vec{X})=\vec{Y}_{m}$, where the elements of $\vec{Y}_{m}$ are the Green's functions and phase advances derived from measurement and the elements of $\vec{Y}$ are the corresponding model quantities which depend on $\vec{X}$ (the BPM gains and cross couplings, the quadrupole strengths, and the sextupole feed-downs). We began the fitting process with reasonable fitting variables, denoted $\vec{x}_{0}$, which were derived from the design (ideal) lattice. Letting $\vec{X}=\vec{x}_{0}+\vec{x}$, we Taylor expanded $\vec{Y}(\vec{X})$, obtaining

$$
\vec{Y}\left(\vec{x}_{0}+\vec{x}\right)=\vec{Y}\left(\vec{x}_{0}\right)+M \vec{x}+\vec{\eta}(\vec{x})=\vec{Y}_{m}
$$

where $M=\frac{d \vec{Y}}{d \vec{x}}\left(\vec{x}_{0}\right)$, and $\vec{\eta}(\vec{x})$ represents the nonlinear terms which grow increasingly negligible with each iteration and can, therefore, be ignored, giving

$$
M \vec{x}=\vec{Y}_{m}-\vec{Y}\left(\vec{x}_{0}\right) \equiv \vec{b}
$$

The least squares solution, $\vec{x}=\left(M^{T} M\right)^{-1} M^{T} \vec{b}$, diverged due to degeneracies, calling for an SVD-enhanced Least-Square fitting process that identified dominant SVD modes [6]. When $\vec{b}$, the residuals, were sufficiently small, the iteration process stopped, and we obtained a model lattice that matched the lattice of the real accelerator. From this lattice, we calculated the one turn transfer matrices from which we extracted critical optical parameters at each BPM location. 


\section{MANIPULATION OF VIRTUAL MACHINE}

Due to high beta beat and linear coupling in LER, we decided to focus on the optics improvement of this machine. Furthermore, the LER model more closely matched the real machine than HER, implying that the LER model would accurately respond to quadrupole adjustment. We again used SVD-enhanced fitting; however, in this case, we, in a sense, fitted in the reverse direction with the fitting relationship $\vec{Y}_{v m}(\vec{Z})=\vec{Y}_{0}$, where $\vec{Y}_{0}$ represents the optical parameters of the desired machine and $\vec{Y}_{v m}$ represents the corresponding quantities of the virtual machine. The response quantities depended on $\vec{Z}$, which represented the strengths of 36 quadrupoles, including linear trombone quads and local and global skews. We defined the fitting residuals as $\vec{Y}_{v m}-\vec{Y}_{0}$. When these residuals became sufficiently low, the iteration process stopped and we obtained a new virtual accelerator that more closely matched the desired lattice. Before fitting started, we were able to weight optical parameters that we considered to be highly critical in our solution. The program, then, placed an increased emphasis on the reduction of the residuals of these parameters and, as a result, other residuals increased. The challenge was to find a combination of parameter weights that decreased important residuals and left the others unchanged. We determined this combination and obtained an improved virtual accelerator with the magnet configuration at the time that BPM data was taken plus the new magnet strengths. We then created a knob, which characterized this new magnet configuration, in the machine control system. The machine operators dialed this knob into the machine and we took more BPM measurements with which we repeated MIA and determined an updated optics characterization. We compared the linear optics parameters 
of the improved real machine to those of the pre-solution machine to gauge the success of our study.

\section{RESULTS}

\section{LINEAR OPTICS OF VIRTUAL ACCELERATOR}

The following LER results were obtained from MIA measurements taken on August 9, 2006. Although HER MIA data were taken on this date, we decided not to focus on this machine, because the HER is currently under planning for a replacement of its $60^{\circ}$ lattice with a $90^{\circ}$ lattice. Thus, our primary focus was shifted to LER. We removed 5 horizontal view and 6 vertical view BPM data from our LER analysis, and the resulting fitting residuals were sufficiently low. Figure 2 compares linear Green’s functions of the model before fitting with those derived from measurement for LER. The figure clearly displays deviations between the two; these deviations were corrected by our fitting process, and the Green’s functions of the resulting model are shown in figure 3. This figure shows an almost perfect match between the measurement-derived Green’s functions and the model Green's functions.

The betatron phase advances are also model response quantities that are useful in determining goodness-of-fit. Figure 4 displays phase advances derived from measurement and phase advances of the starting model (ideal lattice) for LER. Figure 5 shows betatron phase advances after SVD-enhanced fitting. Again, the figures illustrate that we have obtained a model which, according to our BPM measurements, closely resembles the real machine.

After we obtained sufficiently low Green's function and phase advance fitting residuals, we calculated linear optical parameters from one turn maps. Figure 6 compares 
the beta functions of the ideal lattice and our virtual machine. This plot indicates high beta beating in eigenplane 1 . This figure also shows beta functions in the vicinity of IP for the virtual machine and the ideal machine. In virtual LER, $\beta_{x}^{*}=19.6 \mathrm{~cm}$ and $\beta_{y}^{*}=10.1 \mathrm{~mm}$. While the virtual machine $\beta_{y}^{*}$ is reasonably close to that of the ideal lattice, $\beta_{x}^{*}$ is lower due to beta beat. Displayed in figure 7 is a comparison of the coupling parameters between the virtual machine and the ideal lattice. The figure indicates that linear coupling in the model is greater than coupling in the ideal lattice. Strong coupling, in concert with beta beat, increases beam size and consequently degrades luminosity. Therefore, it was our goal to reduce these optical parameters in our model and then apply the new magnet configuration to the real machine.

\section{LINEAR OPTICS OF IMPROVED VIRTUAL ACCELERATOR}

Since the virtual accelerator indicated high beta beat, we placed an appropriately high weight on beta functions in both $\mathrm{x}$ and $\mathrm{y}$ directions during SVD-enhanced fitting of the virtual accelerator to the ideal lattice. Increased weight on beta functions successfully reduced the residuals of this parameter, but increased the residuals of other parameters. We altered the parameter weights and fitted several times before reaching an acceptable solution. In our solution, beta beat was reduced by a factor of $\sim 3$, and other parameters remained within a reasonable range. Figure 8 displays plots of beta functions for the improved virtual accelerator. Note the reduction of beta beat in $\mathrm{x}$ and the increase in the horizontal beta function at IP.

Figure 9 compares linear coupling of the improved machine and the ideal lattice. Since we did not place a high weight on coupling parameters in the fitting process, we 
did not expect a significant change in coupling. Comparing figures 7 and 9, it is apparent that coupling ellipse tilt ratios were in fact reduced in the improved virtual accelerator.

After dialing the improved virtual accelerator into the machine, we repeated model independent analysis and constructed a new virtual accelerator in order to check that our new magnet configuration was indeed successful in improving linear optics. To avoid confusion we will call this virtual accelerator the post-solution virtual machine. Figure 10 displays the beta functions of the post-solution virtual machine. Note the decrease in beta beat, which we predicted with the improved virtual accelerator. The post-solution virtual accelerator indicated an increase in $\beta_{x}^{*}$ that was greater than predicted, and a $10 \%$ increase in $\beta_{y}^{*}$, which agreed with predictions.

\section{DISCUSSION AND CONCLUSION}

In this study, after a systematic removal of noisy BPM data, we successfully constructed a virtual accelerator that matched the linear optics of PEP-II LER. By manipulating the virtual lattice and placing appropriate emphasis on problematic linear optical parameters, we found a magnet configuration that improved the beta beat of the virtual LER. When applied to the real machine, the beta beat decreased by a factor of $~ 3$ as predicted (see figure 10). The new solution yielded an increase in beta functions at IP that was within the range of prediction (compare figures 8 and 10) given that the presolution orbit was not restored after the new magnet configuration was dialed in to the machine. The HER lattice was fine-tuned after the dial in of the new LER solution, and we observed improvement in the performance of the machine. During machine

development on August 16, 2006, PEP-II reached a record peak luminosity of 
$12.069 \times 10^{33} \mathrm{~cm}^{-2} \mathrm{~s}^{-1}$. Furthermore, following the dial in of the new solution, the average luminosity integration increased, and the machine reached the current run goal of $100 \mathrm{fb}^{-1}$ before the anticipated date.

\section{ACKNOWLEDGEMENTS}

I would like to extend my thanks to my advisor, Yiton Yan, for his patience during our many illuminating discussions. I also thank William Colocho for introducing me to my research project and his willingness to help along the way. Finally, I would like to thank Adam Edwards, Stephanie Majewski, and Mike Woods, as well as the Department of Energy Office of Science for providing the opportunity to participate in a rewarding research experience.

\section{REFERENCES}

[1] J. Irwin, C.X.Wang, Y.T Yan, K.L.F. Bane, Y. Cai, F. J. Decker, M. Minty, G.V. Stupakov, F. Zimmermann, "Model-Independent Beam Dynamics Analysis," in Physical Review Letters, Vol. 83, Issue 8, February 1999, pp. 1684-1687.

[2] Y.T.Yan, Y. Cai, F-J., Decker, S. Ecklund, J. Irwin, J. Seeman, M. Sullivan, J. Turner, U. Wienands, "Virtual Accelerator for Accelerator Optics Improvement," SLAC-PUB-11209, presented at PAC 05, May 2005.

[3] Y.T. Yan, Y. Cai, J. Irwin, and M. Sullivan, "Linear Optics Verification for PEPII using Model-Independent Analysis,” SLAC-PUB-9368, presented at 23rd Advanced ICFA Beam Dynamics Workshop on High Luminosity e+ e- Colliders, August 2002.

[4] J. Irwin and Y.T. Yan, "Beamline Model Verification Using Model Independent Analysis,” SLAC-PUB-8515, presented at EPAC 2000, July 2000.

[5] Y.T. Yan, Y. Cai, W. Colocho, F. J. Decker, J. Seeman, M. Sullivan, J. Turner, U. Wienands, M. Woodley, G. Yocky. "Precision Measurement and Improvement of e+,e- Storage Rings,” SLAC-PUB-11925, presented at EPAC 2006, June 2006. 
[6] Y.T. Yan and Y. Cai, "Precision PEP-II Optics Measurement with an SVDEnhanced Least-Square Fitting,” Nuclear Instruments and Methods in Physics Research A, vol. 558, p. 336, 2006.

\section{FIGURES}
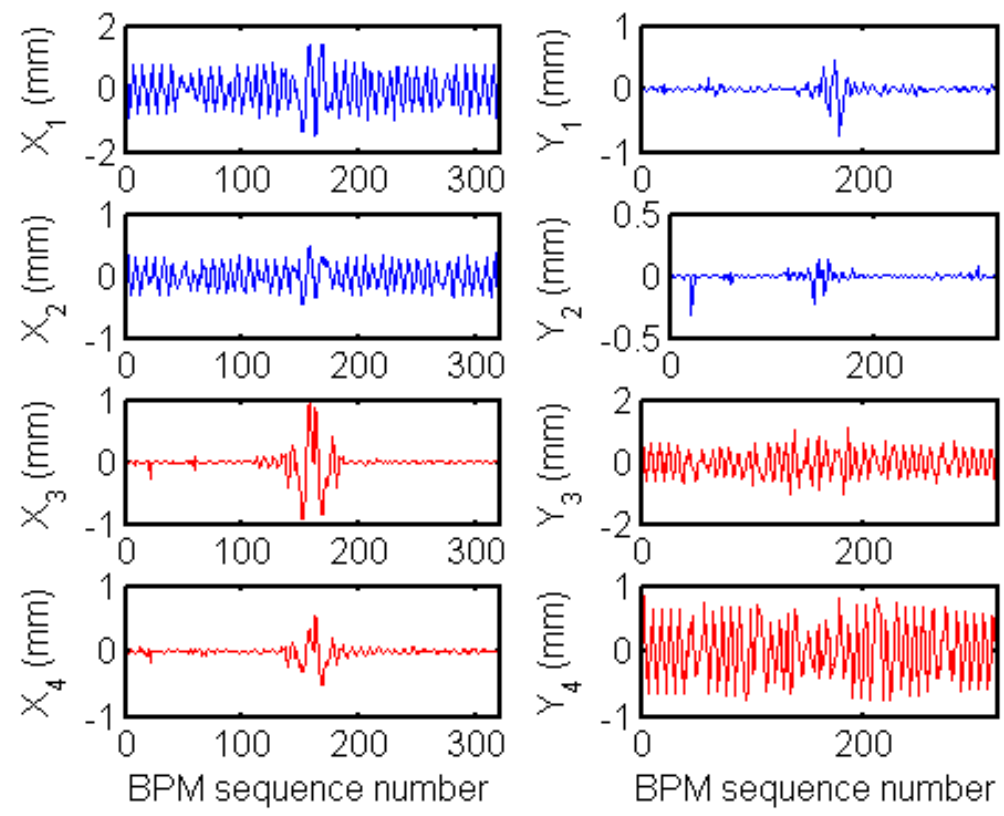

Figure 1 (color). Independent beam orbits from LER on July 27, 2006. Orbits $\mathrm{x}_{1}, \mathrm{y}_{1}, \mathrm{x}_{2}, \mathrm{y}_{2}$ are obtained during horizontal beam excitation. Orbits $\mathrm{x}_{3}, \mathrm{y}_{3}, \mathrm{x}_{4}, \mathrm{y}_{4}$ are obtained during vertical beam excitation. 

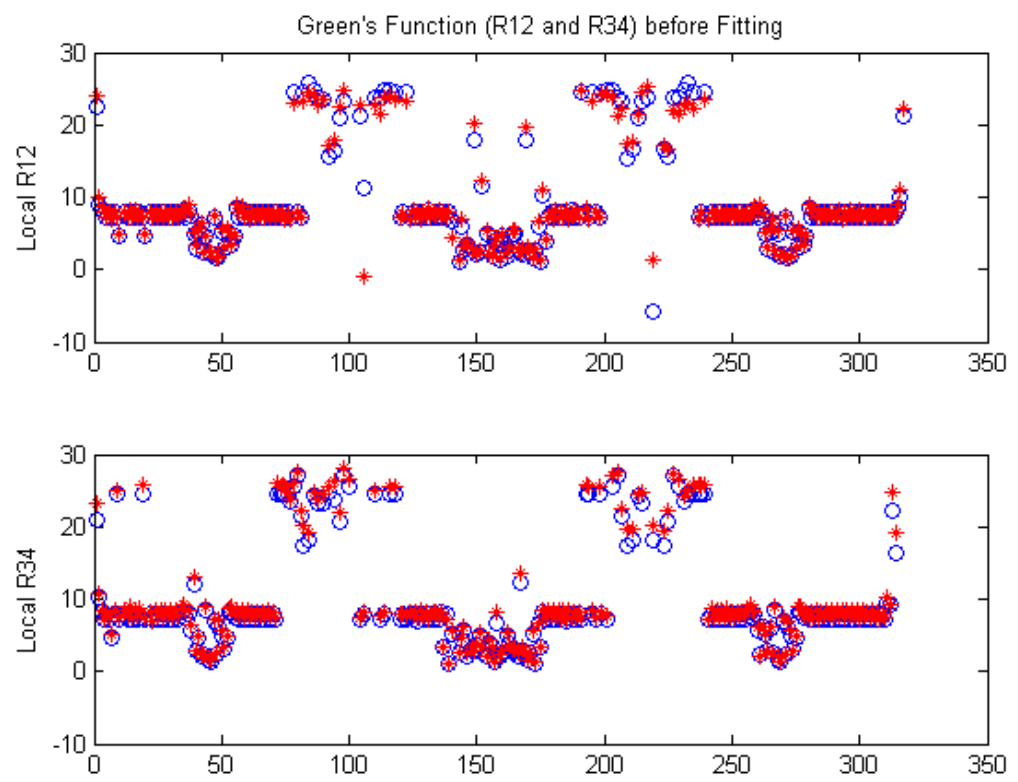

Figure 2 (color). Linear Green's functions obtained from LER. Blue circles represent functions calculated from model before any fitting. Red stars are the functions derived from measurement.
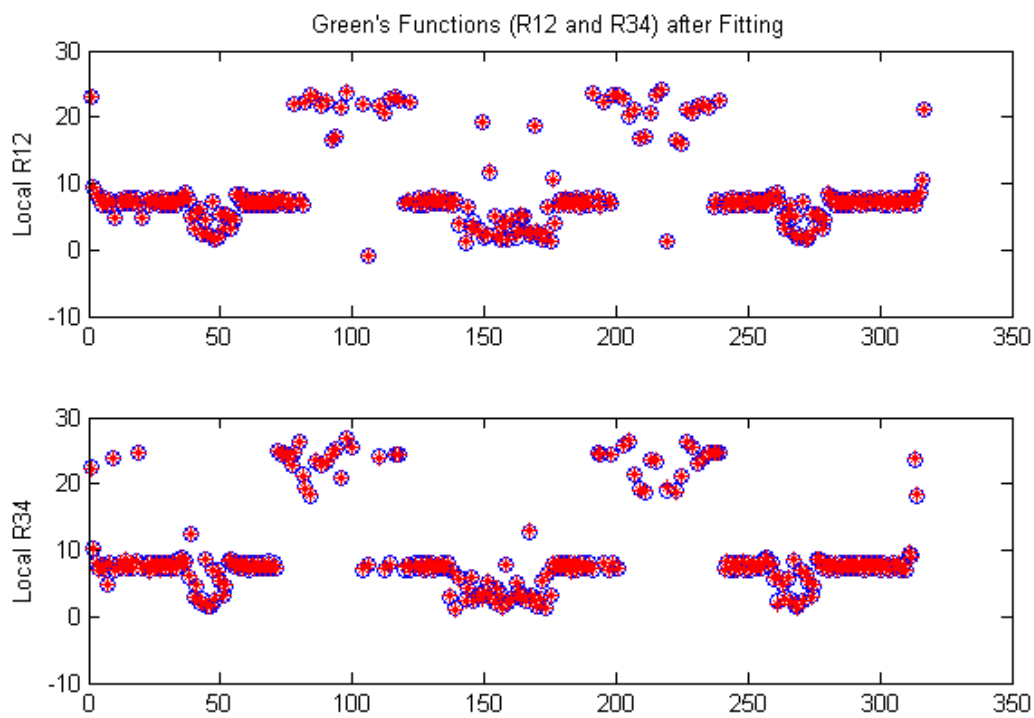

Figure 3 (color). Linear Green's functions obtained from LER. Blue circles represent functions calculated from model after SVD-enhanced fitting. Red stars are the functions derived from measurement. 

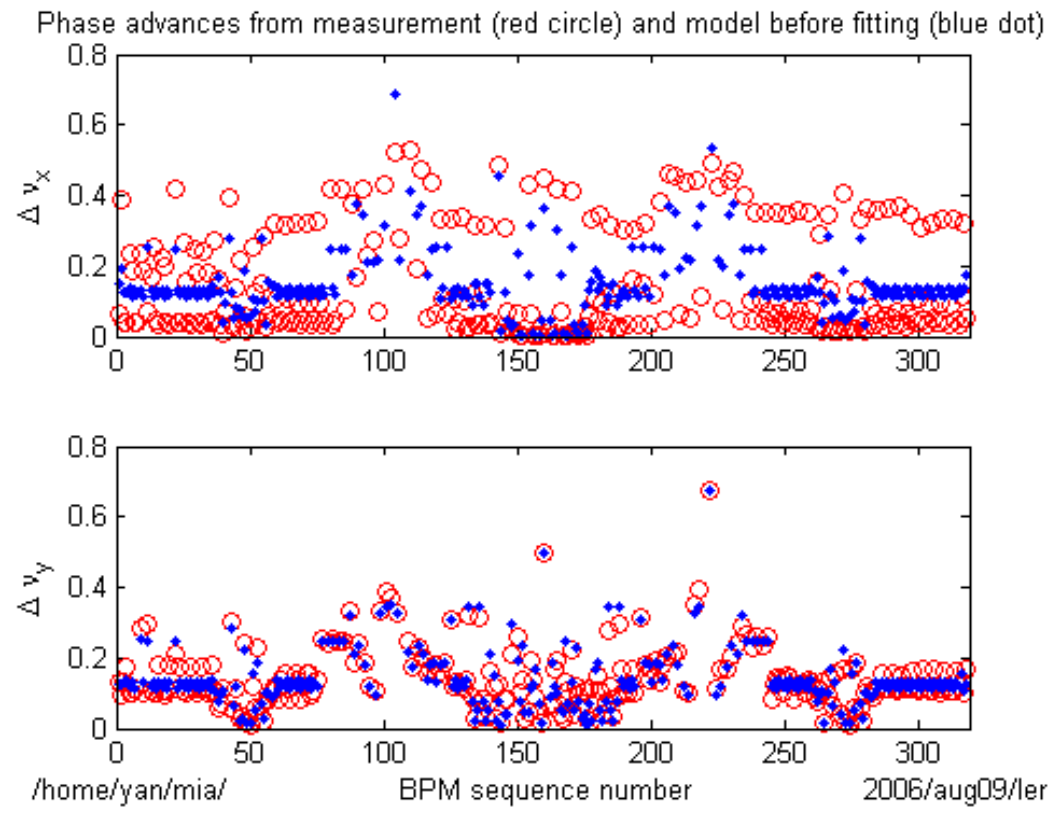

Figure 4 (color). Betatron phase advances obtained from LER before fitting. Red circles represent measured phase advances, while blue dots represent phase advances of model (ideal lattice).
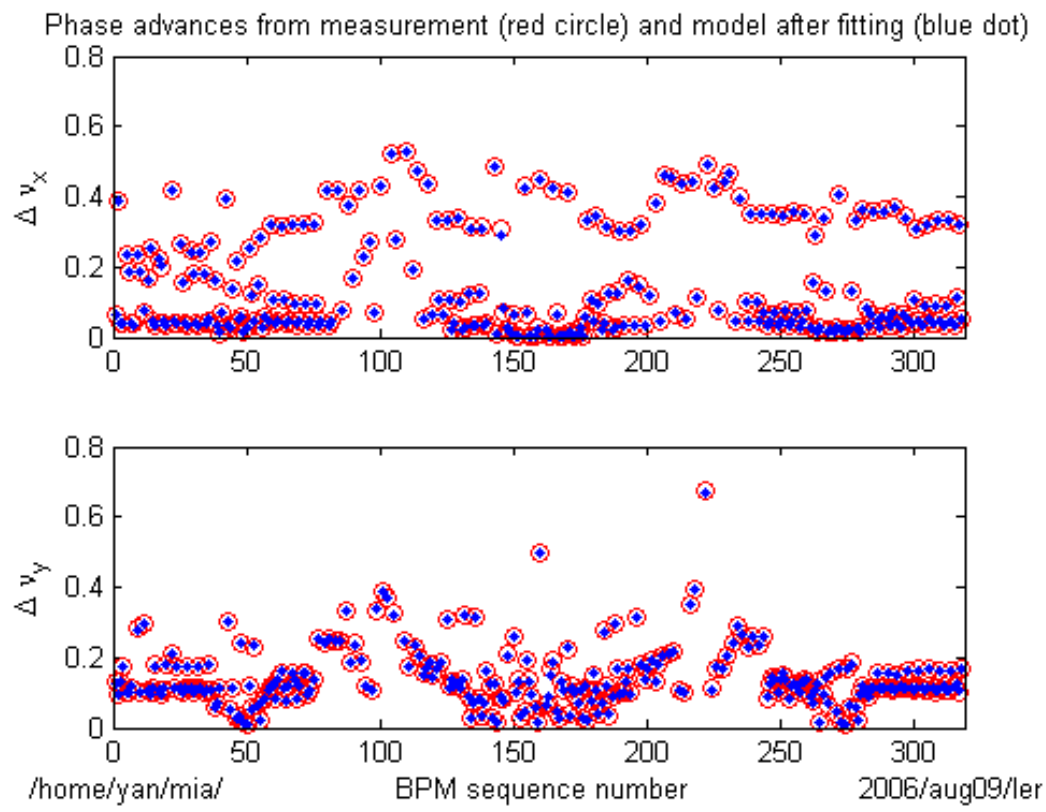

Figure 5 (color). Betatron phase advances obtained from LER after fitting. Red circles represent measured phase advances, while blue dots represent phase advances of model (ideal lattice). 

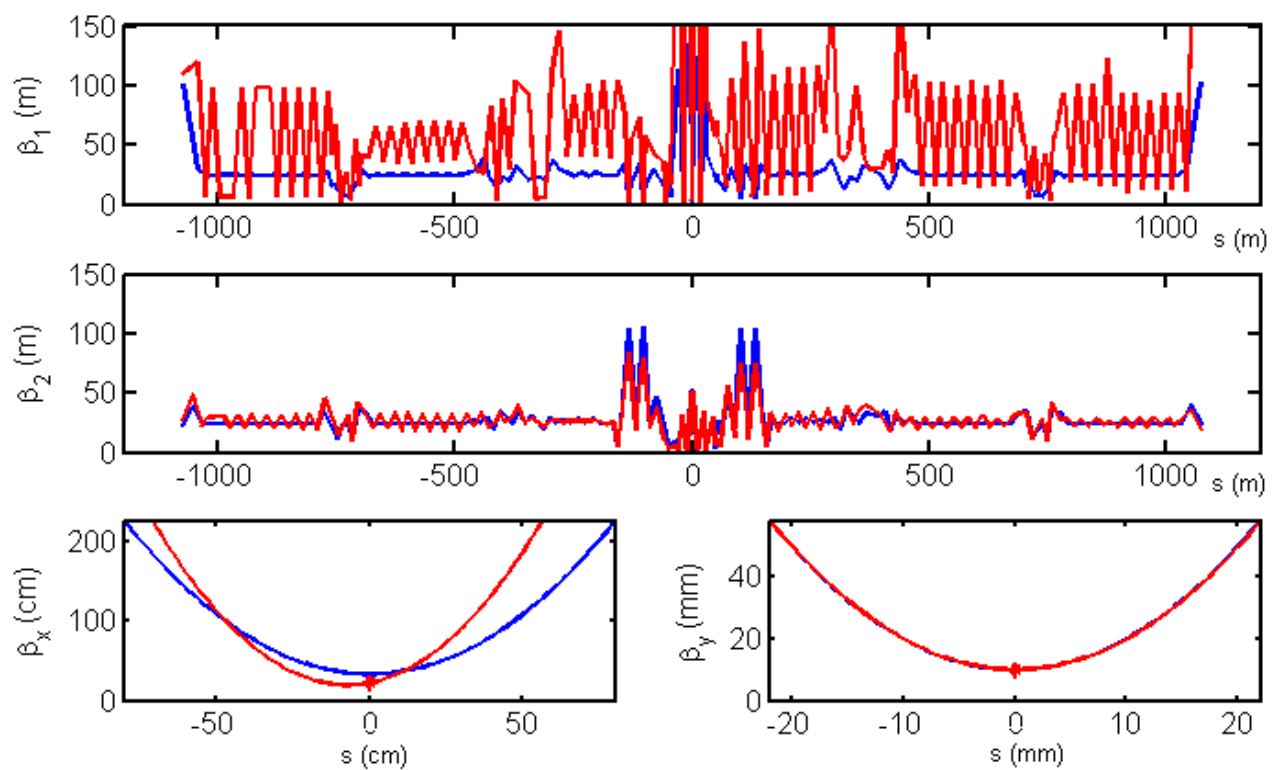

Figure 6 (color). Comparison of virtual machine beta functions (red) and ideal lattice beta functions (blue) for eigenplanes 1 and 2 in LER. Plots on bottom are beta functions at IP. $\beta_{x}^{*}=19.6 \mathrm{~cm}$ and $\beta_{y}^{*}=10.1 \mathrm{~mm}$.
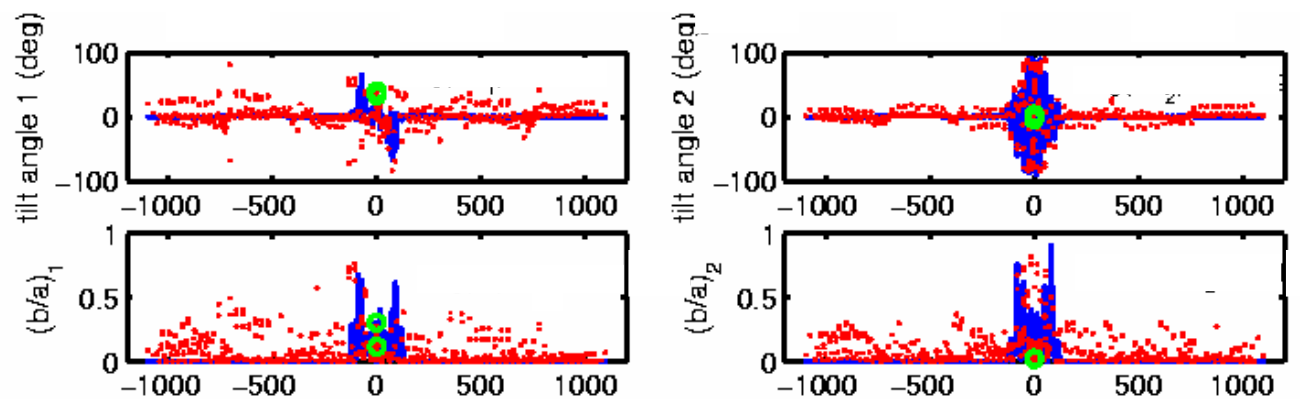

Figure 7 (color). Comparison of linear coupling parameters between model (red) and ideal lattice (blue) in LER. 

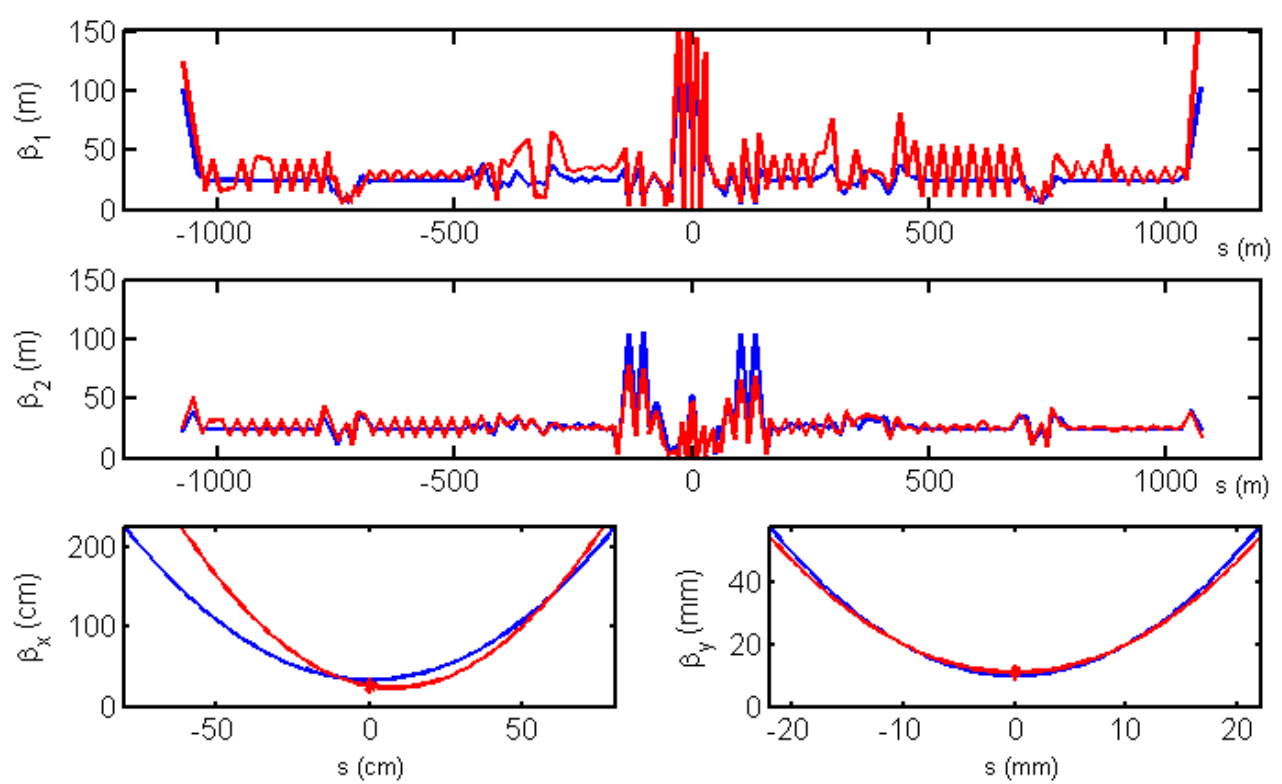

Figure 8 (color). Comparison of improved virtual machine beta functions (red) and ideal lattice beta functions (blue) for eigenplanes 1 and 2. Plots on bottom are beta functions at IP. $\beta_{x}^{*}=37.2 \mathrm{~cm}$ and $\beta_{y}^{*}=10.0 \mathrm{~mm}$.
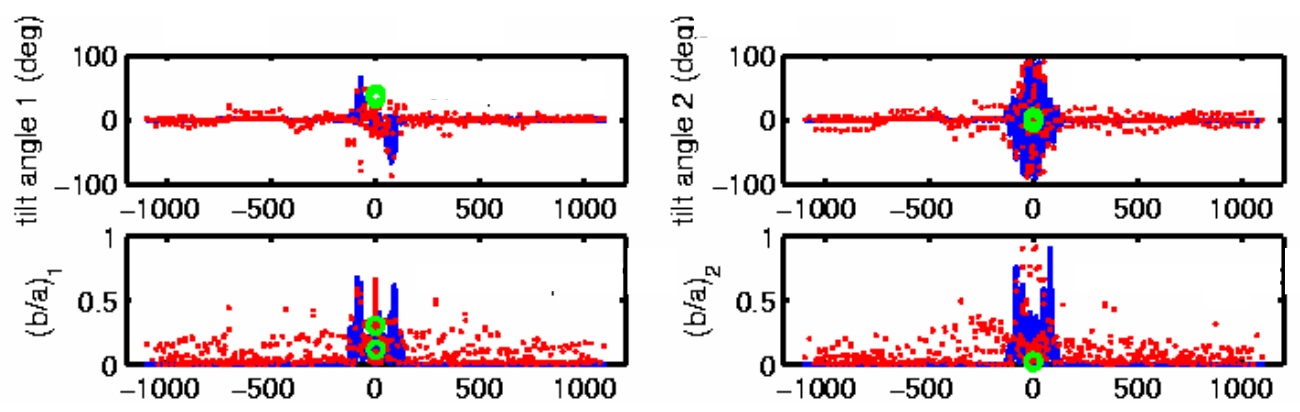

Figure 9 (color). Comparison of linear coupling parameters between improved model (red) and ideal lattice (blue). 

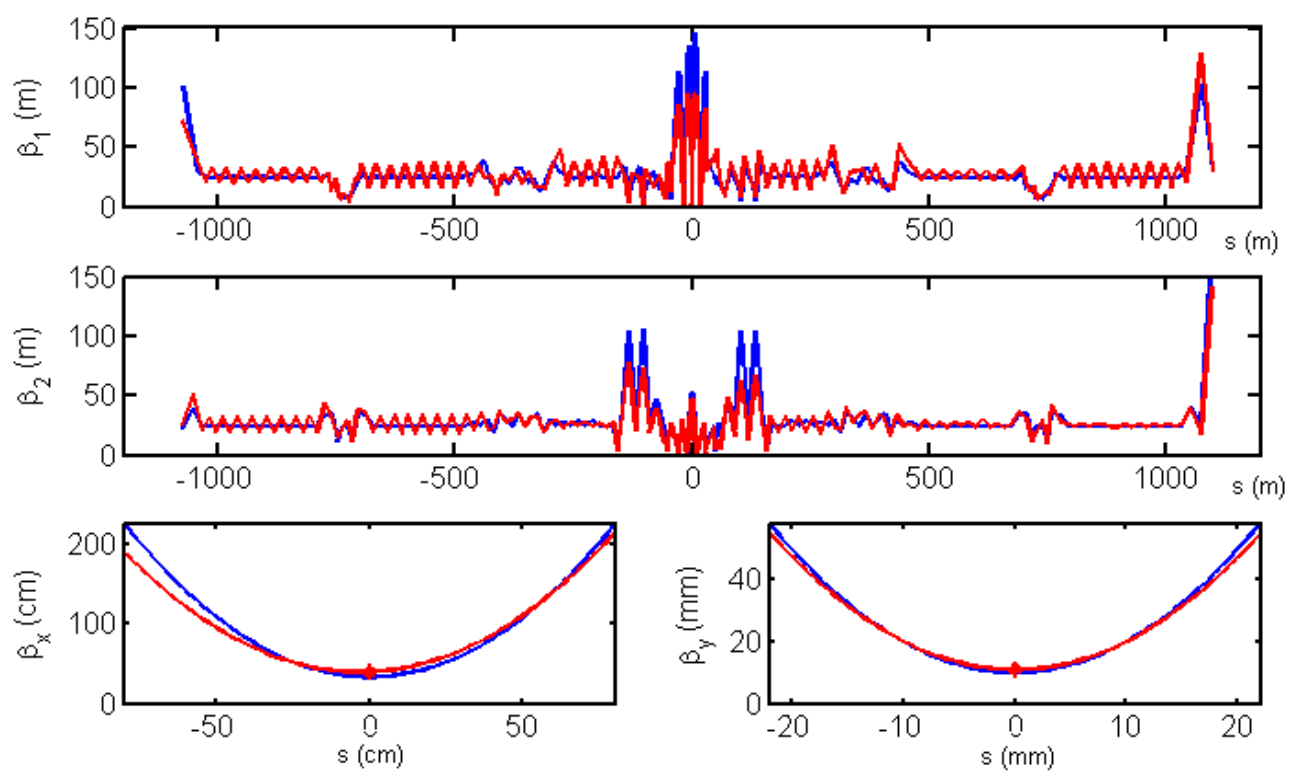

Figure 10 (color). Comparison of post-solution virtual machine beta functions (red) and ideal lattice beta functions (blue) for eigenplanes 1 and 2. Plots on bottom are beta functions at IP. $\beta_{x}^{*}=39.5 \mathrm{~cm}$ and $\beta_{y}^{*}=11.0 \mathrm{~mm}$. 


\title{
Afterglow Radiation from Gamma Ray Bursts
}

\author{
Hugh Desmond \\ Office of Science, Science Undergraduate Laboratory Internship (SULI) \\ Katholieke Universiteit Leuven \\ Stanford Linear Accelerator Center \\ Stanford, CA
}

August 22, 2006

Prepared in partial fulfillment of the requirements of the Office of Science, Department of Energy's Science Undergraduate Laboratory Internship under the direction of Weiqun Zhang at the Kavli Institute for Particle Astrophysics and Cosmology, Stanford Linear Accelerator Center.

Participant:

Signature

Research Advisor:

Signature 


\section{TABLE OF CONTENTS}

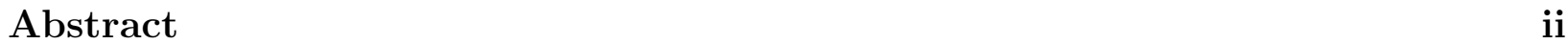

$\begin{array}{ll}\text { Introduction } & 1\end{array}$

Materials and Methods $\quad 2$

$\begin{array}{lr}\text { Results and Discussion } & 6\end{array}$

$\begin{array}{lr}\text { Conclusion } & 7\end{array}$

$\begin{array}{lr}\text { Acknowledgments } & 8\end{array}$

$\begin{array}{lr}\text { References } & 8\end{array}$ 


\begin{abstract}
Afterglow Radiation from Gamma Ray Bursts. HUGH DESMOND (Katholieke Universiteit Leuven, Leuven, Belgium) WEIQUN ZHANG (Kavli Institute for Particle Astrophysics and Cosmology, Stanford Linear Accelerator Center, Stanford, CA 94025)
\end{abstract}

Gamma-ray bursts (GRB) are huge fluxes of gamma rays that appear randomly in the sky about once a day. It is now commonly accepted that GRBs are caused by a stellar object shooting off a powerful plasma jet along its rotation axis. After the initial outburst of gamma rays, a lower intensity radiation remains, called the afterglow. Using the data from a hydrodynamical numerical simulation that models the dynamics of the jet, we calculated the expected light curve of the afterglow radiation that would be observed on earth. We calculated the light curve and spectrum and compared them to the light curves and spectra predicted by two analytical models of the expansion of the jet (which are based on the Blandford and McKee solution of a relativistic isotropic expansion; see Sari's model [1] and Granot's model [2]). We found that the light curve did not decay as fast as predicted by Sari; the predictions by Granot were largely corroborated. Some results, however, did not match Granot's predictions, and more research is needed to explain these discrepancies. 


\section{INTRODUCTION}

The timespan of gamma-ray bursts is relatively short, being between tenths of seconds and one thousand seconds. They are very energetic, despite being short, and they are the brightest known source of gamma-rays in the universe. They even outshine the sun in the gamma-frequency range, which is amazing considering that they occur at cosmological distances, i.e. outside of the Milky Way. It is estimated that a typical GRB releases $10^{52}$ ergs, which is as much energy as all the energy the Sun would radiate during its entire 10 billion year lifetime.

Despite the fact that most energy is released during the first seconds, there is still some emission of radiation after the initial outburst has subsided. There is a residual radiation at $\mathrm{X}$-ray to radio frequencies, which is called the afterglow radiation. This afterglow is much fainter than the initial gamma-ray outburst, and is observable for up to tens of years.

It is a reasonable question to ask what physically causes this big flux of gamma-rays. There is a general consensus that a gamma-ray burst is a witness to a cataclysmic event concerning very compact objects, although the details of the event depend on whether the GRB is 'short' or 'long'. Short GRBs (with durations of $0.1-2 \mathrm{~s}$ ) are thought to be caused by the collision and merging of two very compact objects (such as two neutron stars), which causes a twin jet of plasma to be ejected at highly relativistic speeds along the mutual rotation axis. The jet consists of several shock fronts, moving at different relativistic speeds. When one shock front catches up with another, they collide and energy is emitted in the form of gamma-rays, which we observe. Long GRBs (with durations of $2-1000$ s) are presumably caused by the collapse of a massive star into a black hole, also causing a twin jet of plasma to be shot off along the rotation axis at relativistic energies. These twin jets also emit the gamma radiation we observe.

The afterglow radiation is caused by a mechanism separate from that governing the gamma-ray radiation. When the twin jets hit the surrounding interstellar medium, they cause 
the electrons in that medium to accelerate to relativistic speeds. Then, due to the magnetic field in the plasma jet, these electrons will emit synchrotron radiation. This synchrotron radiation is what will be calculated in this paper.

Studies have already been done on the radiation from GRB afterglows. Early studies simplified the problem, for example by just considering the radiation from one (representative) point [3], or by considering all points, but approximating the dynamics of the jet as the radial expansion of a cone (see [4]), thus neglecting the sidewards expansion. Another

study [5] has tried to take the sidewards expansion into account by making the simplifying assumption that the speed of the sidewards expansion is uniform over $\theta$, the opening angle of the jet. In all these studies these simplifying assumptions were made so an analytical model of the expansion of the jet could be used (this is the Blandford-McKee solution of the ultra-relativistic expansion of a spherical blast wave, see [6]).

In this paper the calculation will be presented based on a numerical model of the dynamics of the jet. This model is a hydrodynamical simulation of how the jet expands (since the jet is a plasma, it shows many similarities with a fluid). The simulation was run by W. Zhang and A. MacFayden on the NASA Colombia supercomputer, and presents a high resolution description of the expansion of the jet. Before going into the light curve and spectrum of the afterglow that we obtained, we will first dwell on the methods we used to calculate the synchrotron radiation.

\section{MATERIALS AND METHODS}

In this section we will break down the formalism into three basic steps. First we will calculate the synchrotron radiation power coming from a single electron. After that, we will calculate the power per unit frequency coming from the jet, which is a distribution of electrons; finally we will transform this emitted power into the observer frame to obtain the observed flux. We will now elaborate on these steps. 
To calculate the radiation emitted by an electron in the jet, it would be sufficient to know what the magnetic field would be at every point and what the speed would be of every single electron. We then would be able to calculate the power through

$$
P\left(\gamma_{e}, \nu\right)=\frac{\sqrt{3}}{2 \pi} \frac{q_{e}^{3} B \sin \alpha}{m_{e} c^{2}} \nu / \nu_{0} \int_{\nu / \nu_{0}}^{\infty} K_{5 / 3}(\eta) d \eta
$$

where $q_{e}$ is the electron charge, $m_{e}$ is the electron mass, $K_{5 / 3}$ is the modified Bessel function of the second kind, $\alpha$ is the angle between the velocity and the acceleration of the electron. The reference frequency $\nu_{0}$ is defined as $\nu_{0}=\frac{3}{2} \frac{q_{e} B \sin \alpha}{m c} \gamma_{e}^{2}$.

However, this microphysics is unknown at present. The hydrodynamical simulation can give us the total internal energy $e_{i n t}$, the particle density $n$ and the bulk velocity of the jet $\boldsymbol{\beta}=\boldsymbol{v} / c$. We make use of this, and make the assumption that the magnetic energy density, $e_{B}$, is a fixed fraction, $\epsilon_{B}$, of the total internal energy density:

$$
e_{B}=\epsilon_{B} e_{i n t}
$$

The strength of the magnetic field can then be easily estimated through $\frac{B^{2}}{8 \pi}=e_{B}$. We make a similar assumption concerning the movement of the electrons within the jet. We assume that the electron energy density, $e_{e}$ is also a fixed fraction, $\epsilon_{e}$ of the total energy density:

$$
e_{e}=\epsilon_{e} e_{i n t}
$$

Then the average gamma factor, $\left\langle\gamma_{e}\right\rangle$ of the electrons can be estimated through $n<\gamma_{e}>m_{e} c^{2}=e_{e}$. For our calculation, we considered an adiabatic case by taking the values $\epsilon_{B}=\epsilon_{e}=0.1$, so that the radiation had a negligible effect on the total energy of the jet.

The estimation of $B$ and $\left\langle\gamma_{e}\right\rangle$ allows us to calculate the power emitted per unit frequency per electron, as stated in equation 1 and visualized in figure 1 . The frequency at which an electron will emit radiation depends on its speed while it is swirled around by the magnetic 
field; that is why a characteristic synchrotron frequency can be associated with a speed and this is done by the relation

$$
\nu\left(\gamma_{e}\right)=\frac{q_{e} B}{2 \pi m_{e} c} \gamma_{e}^{2}
$$

where $q_{e}$ is the electron charge.

To calculate the radiation coming from the jet, we have to make another assumption, this time concerning the specific distribution of the electron energies. We assume that the distribution of the electron energy follows a power-law:

$$
N\left(\gamma_{e}\right) \sim \gamma_{e}^{-p}, \quad \gamma_{e} \geq \gamma_{\min }
$$

where the spectral index $p>2.5$ (in our calculation we used $p=2.5$ ) and $N(\gamma) d \gamma$ is the number of electrons per unit volume with energy between $\gamma m_{e} c^{2}$ and $(\gamma+d \gamma) m_{e} c^{2}$. This energy distribution has been found by previous studies to lead to predictions of the afterglow radiation that fit the observations.

All we need to do now is integrate the power per frequency per electron over the energy distribution to get the power per unit volume, per unit frequency:

$$
P(\nu)=\int_{\gamma_{\min }}^{\infty} P\left(\gamma_{e}, \nu\right) N\left(\gamma_{e}\right) d \gamma_{e}
$$

A slight complication arises in calculating the previous integral because the formula for $P\left(\gamma_{e}, \nu\right)$ (equation 1) is no longer valid when the electrons have a very high gamma factor and thus are rapidly losing a significant fraction of energy ${ }^{1}$. This regime where electrons lose energy rapidly is called the fast cooling region (as opposed to the slow cooling region). The demarcation between the two regions is at the cooling gamma factor, $\gamma_{c}$.

We will not go into the details of the evaluation of this integral; for further reference see [7] for the calculation without the complication of fast cooling and see [1] and especially [8]

\footnotetext{
${ }^{1}$ The synchrotron power emitted goes as $\gamma_{e}^{4}$, whereas the total energy goes as $\gamma_{e}$. So the higher $\gamma_{e}$ is, the faster energy is radiated.
} 
for a discussion of the radiation with fast cooling. When all the electrons are cooling fastly, $\gamma_{\min }>\gamma_{c}$ and we have

$$
P(\nu)= \begin{cases}P_{\nu, \max }\left(\nu / \nu_{c}\right)^{1 / 3} & \nu<\nu_{c} \\ P_{\nu, \max }\left(\nu / \nu_{c}\right)^{-1 / 2} & \nu_{c}<\nu<\nu_{\min } \\ P_{\nu, \max }\left(\nu_{\min } / \nu_{c}\right)^{-1 / 2}\left(\nu / \nu_{\min }\right)^{-p / 2} & \nu>\nu_{\min }\end{cases}
$$

where $\nu_{c}$ is the cooling frequency, and is the frequency associated with the cooling gamma factor through equation 4. Conversely, when $\gamma_{\min }<\gamma_{c}$,

$$
P(\nu)= \begin{cases}P_{\nu, \max }\left(\nu / \nu_{\min }\right)^{1 / 3} & \nu<\nu_{\min } \\ P_{\nu, \max }\left(\nu / \nu_{\min }\right)^{-(p-1) / 2} & \nu_{\min }<\nu<\nu_{c} \\ P_{\nu, \max }\left(\nu_{c} / \nu_{\min }\right)^{-(p-1) / 2}\left(\nu / \nu_{c}\right)^{-p / 2} & \nu>\nu_{\min },\end{cases}
$$

where $P_{\nu, \max }$ is the peak frequency. To complete the second step, we need to integrate $P(\nu)$ over the jet region.

Finally we transform the power into the observer frame. The flux from that cell that reaches an observer on earth is

$$
F\left(\nu^{\prime}, t^{\prime}\right)=\frac{1+z}{4 \pi d_{l}^{2}} \int \frac{P(\nu, t)}{\gamma^{2}(1-\boldsymbol{\beta} \cdot \boldsymbol{n})^{2}} 2 \pi r^{2} \sin \theta d \theta d r
$$

where $d_{l}$ is the luminosity distance (which is, in this case, a measure of the distance to the source of the GRB), $z$ the redshift and $\boldsymbol{n}$ a unit vector pointing towards the observer (on the rotation axis). The primed quantities $t^{\prime}$ and $\nu^{\prime}$ denote the observer frame time and frequency. The relation between source and observer frequency is

$$
\nu=\gamma(1-\boldsymbol{\beta} \cdot \boldsymbol{n})(1+z) \nu^{\prime}
$$


which takes into account the redshift and doppler shift ${ }^{2}$. The relation between source and observer time is

$$
t=t^{\prime}-\frac{r \cos \theta}{c}
$$

and accounts for the transit time of the flux.

\section{RESULTS AND DISCUSSION}

Figure 2 shows the calculated light curves for 8 representative frequencies, ranging from radio frequencies to optical and x-ray frequencies. The light curve of each frequency roughly follows a segmented power-law.

The break time is represented by the vertical dotted line. At that time, the jet no longer expands purely radially, but expands in a sidewards direction as well. One consequence of this is that the volume occupied by the jet increases more sharply, leading to a sharper drop in internal energy and a sharper drop in radiation emitted. Another consequence is that at that time all of the jet is seen and there is no further increase in the observed area of the jet. To understand this, consider the radiation of charged particles moving at relativistic speeds; the emitted photons are beamed, i.e. concentrated in a cone with an angle of $\theta_{\text {rad }} \sim 1 / \gamma$, where $\gamma$ is the gamma factor of the moving particle. So when the a part of the forefront of the jet is moving at relativistic speeds in radial direction, the radiation is concentrated in a cone along the direction of the speed. As the jet slows down, the radiation is less concentrated, so the radiation from a increasingly large area of the jet actually reaches the observer. When the jet finally breaks, $\theta_{\text {rad }} \approx \theta_{\text {jet }}$, where $\theta_{\text {jet }}$ is the opening angle of the jet, and the observer can see the radiation coming from all areas of the jet. Thus there will be no contribution from the fact that radiation from an increasingly large area is reaching the observer; the subsequent drop in radiation will therefore be sharper than before the jet break.

Before the break, the flux goes as $t^{1 / 2}$ for low frequencies and as $t^{(2-3 p) / 4}$ for high fre-

\footnotetext{
${ }^{2}$ Note that $\gamma=\left(1-\beta^{2}\right)^{-1 / 2}$ is the bulk gamma factor of the jet and is not the same as the $\gamma_{e}$, the gamma factor of an electron.
} 
quencies. After the break, all frequencies go as $t^{-p}$. At later times, we find that the decrease in flux is less steep than predicted by the analytical model. This is partially due to the fact that we have taken the effect of the counter-jet into account. At later times, the radiation coming from the counter-jet is visible, and this accounts for the 'bump' in the flux at around 5000 days.

If we compare the behavior of the flux after the break with the predictions of Sari's analytical model, see [1], and which is represented in the figure by the dotted lines, we find that our calculated flux shows a decay that is significantly steeper than the decay predicted by Sari, both for low frequencies and high frequencies. However, there is quite a good agreement with Granot's analytical model, which is represented by the dash-dot lines. This result thus corroborates Granot's model at the expense of Sari's model. As mentioned above, at later times the decay does seem as rapid as predicted by Granot's model, even when we account for the contribution from the counter-jet.

The spectrum of the afterglow is represented in figure 3. The break in the power-law occurs at $\nu=\nu_{c}$. The cooling frequency shifts to higher frequencies at later times, which is reasonable, because it means that fewer electrons are in the fast cooling region as time progresses. In general, the calculated spectrum agrees extremely well with the predictions of both analytical models.

\section{CONCLUSION}

We have calculated both the observed light curves and spectra from the afterglow radiation. In the calculation we used data from a numerical hydrodynamical simulation of the jet, and assumed a power-law distribution of electron energy. Furthermore we made assumptions concerning the microphysics of the jet so that we could estimate the magnetic field and the movement of the electrons within the jet.

We compared the light curve to Sari's model and found the decay of the light curve to be 
significantly different. In contrast, the calculated light curve agreed very well with Granot's predictions. The calculated spectrum agreed well with both Sari's and Granot's predictions.

More research is needed to fully explain all the features of the results, such as the discrepancy at later times between our calculated light curve and Granot's prediction. More research is also needed to fully exploit the numerical simulation. We did the calculation for a jet where the energy and density are distributed uniformly over the opening angle. This is not realistic, and in the future the calculation could be done for a jet where the energy and density follow a non-uniform distribution (such as a Gaussian distribution).

\section{ACKNOWLEDGMENTS}

I would foremost like to thank my mentor, Weiqun Zhang, for his help and patient explanations during my research this summer. Thanks also to Josh Kline, whose witty and insightful comments have been instrumental to this paper. I would like to thank the U.S. Department of Energy, Office of Science for giving me such a generous opportunity to live and breathe

physics at the Stanford Linear Accelerator Center for a whole summer. Special thanks to Mike Woods, Pauline Wethington, Adam Edwards and Stephanie Majewski, who spent a lot of time organizing the program and who made everything possible.

\section{REFERENCES}

[1] R. Sari, T. Piran and R. Narayan, Spectra and Light Curves of Gamma-Ray Burst Afterglows, The Astrophysical Journal, 1998, pp. 17-20.

[2] J. Granot, Afterglow Light Curves from Impulsive Relativistic Jets with an Unconventional Structure, The Astrophysical Journal, 2005, pp. 1022-1031.

[3] P. Mészáros and M. J. Rees, Optical and Long-Wavelength Afterglow from Gamma-Ray Bursts, Astrophysical Journal, pp. 232-237. 
[4] J. Granot, T. Piran and R. Sari, Images and Spectra from the Interior of a Relativistic Fireball, The Astrophysical Journal, 1999, pp. 679-689.

[5] J. E. Rhoads, The Dynamics and Light Curves of Beamed Gamma-Ray Burst Afterglows, The Astrophysical Journal, 1999, pp. 737-739.

[6] R.D. Blandford and C.F. McKee, Fluid Dynamics of Relativistic Blast Waves, The Physics of Fluids, Vol. 19, 1976.

[7] G. B. Rybicki and A. P. Lightman, Radiative processes in Astrophysics, John Wiley \& Sons, 1979, pp. 167-191.

[8] T. Piran, The physics of gamma-ray bursts, Reviews of Modern Physics, vol. 76, Issue 4, pp. 1164-1167. 


\section{FIGURES}

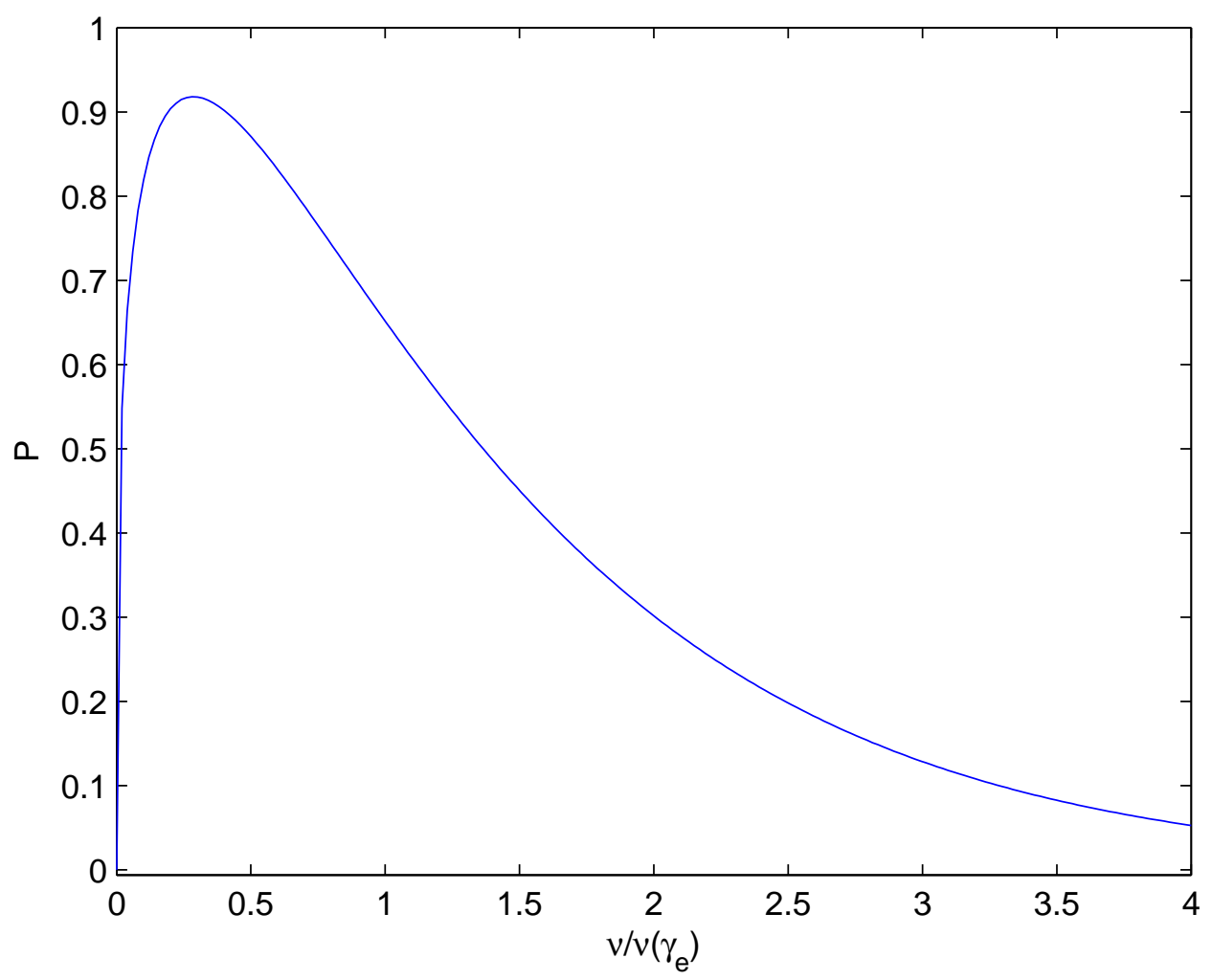

Figure 1: Synchrotron radiation of an electron. The power goes as $\left(\nu / \nu\left(\gamma_{e}\right)\right)^{1 / 3}$ for $\nu \ll \nu\left(\gamma_{e}\right)$ and drops exponentially for $\nu \gg \nu\left(\gamma_{e}\right)$. 


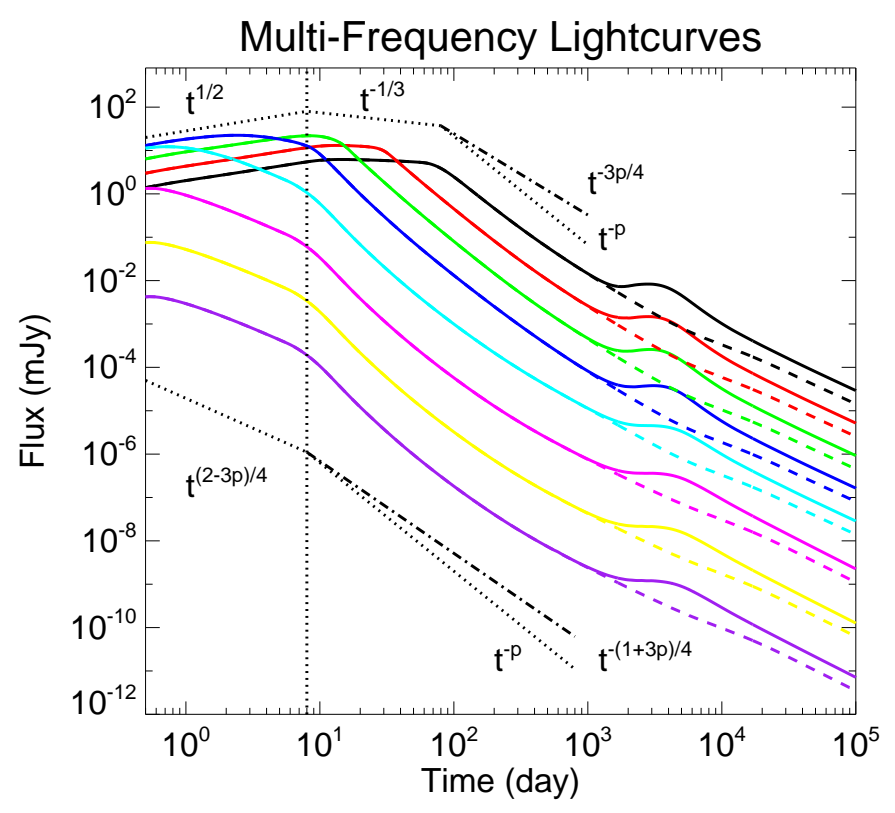

Figure 2: The calculated light curves per frequency are represented by the solid lines. The frequencies range from $10^{9} \mathrm{~Hz}$ for the black line, to $10^{18} \mathrm{~Hz}$ for the violet line. The dashed line is the calculated flux without taking the contribution from the counter jet into account. The dotted lines are the light curves predicted by Sari's model, whereas the dash-dot lines are the light curves as predicted by Granot's model. The dotted vertical line represents the break time.

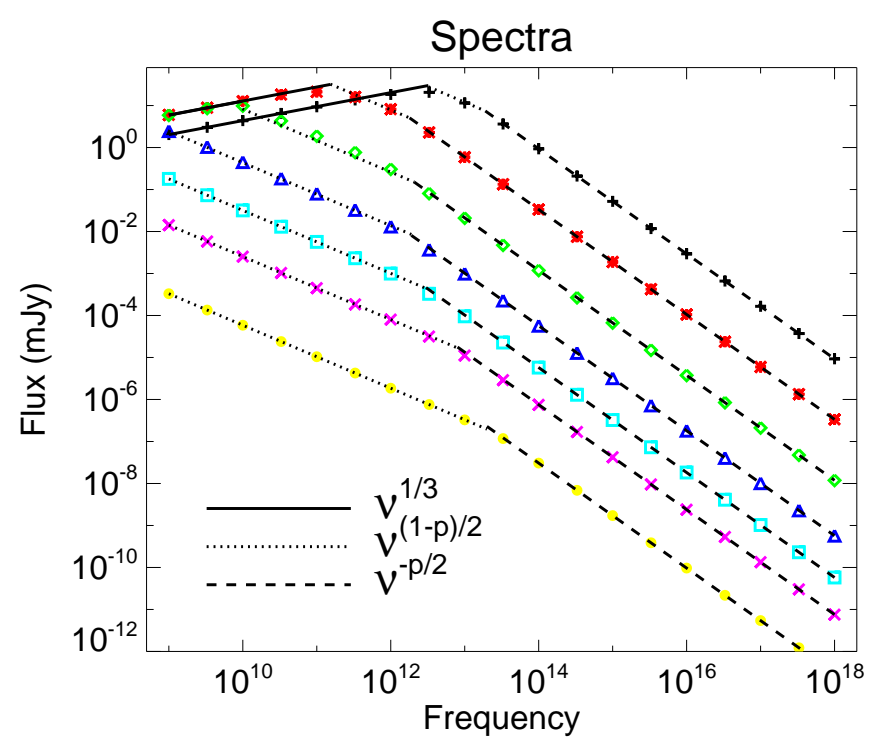

Figure 3: The colored dots represent the fluxes calculated in this study. The predictions from the analytical models are shown in the dotted lines. 


\title{
Characterization of an Electromagnetic Calorimeter for the Proposed International Linear Collider
}

\author{
Merideth Frey \\ Office of Science, SULI Program \\ Wellesley College \\ Stanford Linear Accelerator Center \\ Menlo Park, California
}

August, 242006

Prepared in partial fulfillment of the requirements of the Office of Science, U.S. Department of Energy’s Science Undergraduate Laboratory Internship under the direction of Dr. Norman Graf and Dr. Tony Johnson in the International Linear Collider group of the High Energy Physics division at Stanford Linear Accelerator Center.

Participant:

Signature

Research Advisor:

Signature 


\section{Table of Contents}

Abstract 3

$\begin{array}{ll}\text { Introduction } & 4\end{array}$

Materials and Methods $\quad 5$

$\begin{array}{ll}\text { Results } & 7\end{array}$

$\begin{array}{lr}\text { Discussion and Conclusion } & 9\end{array}$

$\begin{array}{ll}\text { Acknowledgements } & 12\end{array}$

$\begin{array}{ll}\text { References } & 12\end{array}$

$\begin{array}{ll}\text { Tables } & 14\end{array}$

$\begin{array}{ll}\text { Figures } & 15\end{array}$ 


\begin{abstract}
Characterization of an Electromagnetic Calorimeter for the Proposed International Linear Collider. MERIDETH FREY (Wellesley College, Wellesley, MA 02481) NORMAN GRAF (Stanford Linear Accelerator Center, Menlo Park , CA 94025) TONY JOHNSON (Stanford Linear Accelerator Center, Menlo Park , CA 94025).
\end{abstract}

The International Linear Collider (ILC) is part of a new generation of accelerators enabling physicists to gain a deeper understanding of the fundamental components of the universe. The proposed ILC will accelerate positrons and electrons towards each other with two facing linear colliders, each twenty kilometers long. Designing and planning for the future accelerator has been undertaken as a global collaboration, with groups working on several possible detectors to be used at the ILC. The following research at the Stanford Linear Accelerator Center (SLAC) pertained to the design of an electromagnetic calorimeter. The energy and spatial resolution of the calorimeter was tested by using computer simulations for proposed detectors. In order to optimize this accuracy, different designs of the electromagnetic calorimeter were investigated along with various methods to analyze the data from the simulated detector. A low-cost calorimeter design was found to provide energy resolution comparable to more expensive designs, and new clustering algorithms offered better spatial resolution. Energy distribution and shape characteristics of electromagnetic showers were also identified to differentiate various showers in the calorimeter. With further research, a well-designed detector will enable the ILC to observe new realms of physics. 


\section{INTRODUCTION}

Particle detectors have changed dramatically from early cloud chambers into the elaborate multi-layered devices used today. With new higher energy colliders revealing particles never seen before, detectors need to be as accurate as possible in order to determine properties of these new particles. The ILC provides an opportunity for physicists to design and build precise detectors that are not useful for present accelerators such as the Large Hadron Collider (LHC). Since electrons and positrons are fundamental particles - unlike the hadrons that will be collided at the LHC - the ILC will involve less complicated particle transformations, making the use of more precise instruments practical [1]. With higher energy collisions and well-designed detectors, the ILC will provide results to further illuminate physics at the fundamental particle level.

Scientists around the world are working together to design the ILC. At the Stanford Linear Accelerator Center (SLAC), the Linear Collider group is designing an electromagnetic (EM) calorimeter - the component of the ILC particle detector optimized to measure the energy and position of electrons and gamma ray photons. The proposed EM calorimeter is heterogeneous and made of multiple layers of materials: a dense metal with which particles interact and lose energy to forming new particles, and an active material which produces a detectable signal dependent upon the amount of energy absorbed. This type of calorimeter only directly measures a small percentage of the particle energy, but since active material is located evenly throughout the calorimeter, researchers can statistically find the overall energy absorbed. Using a heterogeneous calorimeter enables researchers to analyze the signal using various statistical methods and to find an optimal dense metal absorber for the particular calorimeter. In 
this study, heterogeneous calorimeters with different numbers of layers and various thicknesses of dense metal absorbers were analyzed to optimize energy resolution.

Along with the many possible physical components that can be optimized to increase accuracy for a given calorimeter, methods to analyze the data recorded by the detector can also be investigated. When a particle collides with the calorimeter, it produces many other particles which also collide with the calorimeter material, creating what is called a particle shower. When a collision occurs in the active material (referred to as a hit), the position and energy is recorded. This energy is then corrected using statistical analysis to include the energy lost in the passive material. The energy of the original particle is measured by grouping all the hits from the showered particles (referred to as a cluster) and summing their energy. However, deciding what hits belong to what particle is complicated; especially if two showering particles hit the calorimeter close to each other at around the same time. For this reason, different clustering algorithms have been written to try to improve the resolution of two nearby particles and their effectiveness needs to be tested.

Other valuable analyses involve differentiating between electromagnetic showers showers resulting from an electron or photon colliding with the calorimeter - and showers resulting from other particles that interact with the calorimeter. Electromagnetic showers have many identifying features in their shape and energy distribution. Determining these characteristics can help identify relevant electromagnetic showers from the calorimeter data.

\section{MATERIALS AND METHODS}

The software used to simulate the calorimeter for various events was Simulator for the Linear Collider (SLIC) [2] written by Jeremy McCormick who works in the Linear Collider group at SLAC and based the program on the Geant4 simulation toolkit [3]. This software produces data of detected hits from multiple collisions for a given detector which can be 
analyzed using the Java Analysis Studio (JAS). JAS is an open-source, data analysis tool which provides a graphical user interface to load and compile user Java programs. For plotting data, JAS implements Abstract Interfaces for Data Analysis (AIDA). There is also a built-in event display called WIRED4 - see Figure 1 - which allows the user to view the hits and tracks of particles resulting from a collision at any desired angle and display information about any selected item. The Linear Collider Simulator (LCSim) Event Browser displays all the information known about different hits and tracks of particles from a given simulated collision and was mostly used to double-check the plots.

In order to determine the accuracy of the EM calorimeter at measuring the energy of a particle, single particles of known energy and position were used and the energy resolution of three different calorimeter designs was analyzed. One design had thirty layers of the same absorbent material thickness (referred to as homogeneous thirty layer calorimeter), another had forty layers of the same absorbent material thickness (referred to as homogeneous forty layer calorimeter), and the final design had twenty layers of one absorbent material thickness and then ten layers with the absorbent material twice as thick (referred to as heterogeneous thirty layer calorimeter). The absorbent material used for these designs had $95 \% \mathrm{~W}, 4.4 \% \mathrm{Ni}$, and $0.6 \% \mathrm{Fe}$. See Table 1 for the absorbent material thickness for each of the calorimeter designs. Figure 2 shows a diagram of one layer in the calorimeter. Single photons were also used to identify characteristics of electromagnetic showers by examining the photons' shower shapes and energy distributions.

To determine how well the calorimeter could resolve two nearby particles, analyses were done using two equal-energy photons with various opening angles. Spatial resolution is highly dependent on the type of clustering algorithm used to determine what hits came from the same particle, so various clustering algorithms were analyzed. The simplest clustering algorithm - 
called the nearest neighbor - groups all nearby hits into the same cluster. The directed tree algorithm starts clustering at the first layer of the calorimeter and proceeds to higher layers. This method groups hits into the same clusters as the nearby hits in the previous layers. This means that two clusters which overlap in the calorimeter - and would be declared one cluster by the nearest neighbor algorithm - can be differentiated if they both are distinct clusters in the first layer. The fixed cone algorithm finds the highest energy hits in the calorimeter and forms cones around these hits with vertex at the collision point - see Figure 3. It then groups all the hits within a cone as part of one cluster.

All analyses were done using programs written in Java and plotted using AIDA through JAS. All measurements were found by analyzing many events ( 5000) and fitting histograms with a Gaussian or taking the mean. The heterogeneous thirty layer calorimeter was used unless otherwise specified.

\section{RESULTS}

Figures 4, 5, and 6 are plots of the total energy found in the EM calorimeter for a single particle hitting the barrel perpendicularly versus the known energy of the particle using the homogeneous thirty layer calorimeter, homogeneous forty layer calorimeter, and heterogeneous thirty layer calorimeter, respectively. Table 2 gives the values of the constants found from a linear fit of the data for each of the calorimeter designs.

Figure 7 shows a plot of the total cluster energy found for a single $20 \mathrm{GeV}$ photon when varying the input parameters for the nearest neighbor clusterer. The input parameters include the minimum number of hits to form a cluster (minCell) and three parameters that determine how nearby a hit must be to be included in the cluster (dlayer, dU, and dV). The parameter dlayer defines the maximum number of layers away from the cluster a hit can be to be included in the cluster. The parameters $\mathrm{dU}$ and $\mathrm{dV}$ define the maximum distance from the cluster a hit can be in 
either direction within the layer to be included in the cluster. The parameter minCell was kept constant at five and dlayer was kept constant at one. Since photon clusters are known to have circular cross-sections, $\mathrm{dU}$ and $\mathrm{dV}$ were kept equal, but the magnitude for $\mathrm{dU}=\mathrm{dV}$ was varied.

Figures 8, 9, and 10 are energy resolution plots for single photons using the homogeneous thirty layer calorimeter, homogeneous forty layer calorimeter, and heterogeneous thirty layer calorimeter, respectively. For sampling calorimeters, the intrinsic energy resolution $(\sigma / E)$ can be approximated by:

$$
\sigma / \mathrm{E} \approx \mathrm{p} 1 / \mathrm{sqrt}(\mathrm{E})+\mathrm{p} 0
$$

where $\mathrm{p} 1$ and $\mathrm{p} 0$ are constants, $\mathrm{E}$ is the mean of the energy distribution, and $\sigma$ is the standard deviation (see [4] for a more thorough analysis of energy resolution for sampling calorimeters). By plotting $y=\sigma / E$ and $x=s q r t(E)$ and fitting a line to the data, the constants $p 1$ and $p 0$ were found. The nearest neighbor algorithm was used for clustering, with minCell $=5$ and $d U=d V=$ dlayer $=1$. In order to analyze only the cluster formed by the incident photon, the maximumenergy cluster of each event was used to find $\mathrm{E}$ and $\sigma$. Table 3 gives the values of $\mathrm{p} 1$ and $\mathrm{p} 0$ found from a linear fit of the data for each of the calorimeter designs.

Figures 11 and 12 show the total energy and approximated width for a photon cluster in each layer of the electromagnetic calorimeter. Single particle events were used with $20 \mathrm{GeV}$ photons. The clusters were found using the nearest neighbor algorithm, with minCell $=5$ and $\mathrm{dU}$ $=\mathrm{dV}=$ dlayer $=1$. Only clusters with high energy were analyzed so that the many low-energy clusters (usually isolated hits) would not ruin the analysis. This was accomplished by comparing the cluster energy to a threshold energy, usually about $15 \%$ of the known energy of the incident photon. For each high-energy cluster, the total energy in each layer was found and plotted. The width in each layer was approximated by finding the maximum distance from a hit in the cluster 
to the centroid of the cluster along the z-direction (parallel to the axis of the cylindrical detector) and multiplying this value by two.

Figures 13, 14, and 15 show the shape parameters (similar to moments of inertia about three perpendicular axes using energy instead of mass) for a single photon cluster versus energy

of the photon. Different input parameters of the nearest neighbor clustering algorithm were used to see how this affected the shape of the cluster. The parameter minCell was kept constant at five, while $\mathrm{dU}=\mathrm{dV}=$ dlayer was varied.

Figure 16 shows the mean number of clusters detected for two $1 \mathrm{GeV}$ photons with various opening angles using the clustering algorithms: nearest neighbor, directed tree, and fixed cone. Only high-energy clusters were analyzed, and low-energy clusters from isolated hits were filtered out by use of a threshold energy ( $50 \%$ of known photon energy).

\section{DISCUSSION AND CONCLUSION}

The energy resolution properties of the simulated detector can be seen in Figures 4 through 10. The plots of the total energy in the EM calorimeter versus the known energy of the photon for single photon events (Figures 4, 5, and 6) all have linear fits with slopes near to one and small y-offsets (Table 2). As expected, the most expensive design, the homogeneous forty layer calorimeter, performed the best, having a slope of $\sim 0.997$ and an offset of $\sim-0.00336$. The least expensive design, the homogeneous thirty layer calorimeter, performed the worst with a slope of $\sim 0.979$ and an offset of $\sim 0.0170$. The heterogeneous thirty layer calorimeter (a cheaper alternative to the forty layer design) has a slope of $\sim 0.982$ and an offset $\sim 0.0146$, making this design better than the homogeneous thirty layer calorimeter but slightly worse than the more expensive homogeneous forty layer calorimeter. Note that these plots use all the energy detected in the EM calorimeter, and these results are less ideal when clustering is used. 
Figure 7 shows how changing the input variables of the nearest neighbor clustering algorithm can alter energy resolution. Increasing $\mathrm{dU}$ and $\mathrm{dV}$ increases the total cluster energy because more hits are being included. However, this energy appears to plateau for higher values of $d U$ and $d V$, suggesting that eventually increasing $d U$ and $d V$ may cease to be useful for increasing the total cluster energy. For more many particle events, this increase of neighborhood decreases the spatial resolution of showers in the detector because nearby showers may be grouped into one cluster. Further study to find the ideal values for $\mathrm{dU}$ and $\mathrm{dV}$ to increase energy resolution without losing spatial resolution would be beneficial.

Further energy resolution analyses for photons of different energies are shown in Figures 8, 9, and 10. Ideally, the intrinsic energy resolution should be smaller than $20 \%$ of the energy of the particle. For the plots in the figures, the linear fit would have no y-offset and a very low slope. All the calorimeter designs had very similar results (Table 3). Surprisingly, the homogeneous thirty layer calorimeter has the lowest slope and y-offset but the highest $\chi^{2}$ value and the homogeneous forty layer calorimeter has the highest slope and y-offset but the lowest $\chi^{2}$ value. The heterogeneous thirty layer calorimeter has a low slope within the error bounds of the homogeneous thirty layer calorimeter and a lower $\chi^{2}$ value. With these results along with the linearity results, the heterogeneous calorimeter appears to provide a good blend of the thirty and forty layer homogeneous calorimeters without a huge cost increase from the cheaper homogeneous thirty layer calorimeter.

Characteristics of electromagnetic showers, using the nearest neighbor clustering algorithm, are shown in Figures 11 through 15. As can be seen in Figures 11 and 12, electromagnetic showers on average have a very specific energy distribution and width throughout the layers of the calorimeter. This information can be used to differentiate electromagnetic showers from other showers that may occur in the detector. The heterogeneous 
thirty layer calorimeter was used, and the higher absorbency - due to the thicker absorbent material in the last ten layers of the calorimeter - causes the energy measurements to increase dramatically for layers twenty-one through thirty in Figure 11.

The shape parameters plotted in Figures 13, 14, and 15 also show a distinct shape for electromagnetic showers. Shape parameter one is much smaller than shape parameters two and three, which are very similar in magnitude. These shape parameters suggest a shower shape similar to an elongated ellipsoid with a circular cross-section. Of course, the magnitudes of the shape parameters change depending on energy and the input parameters used for the nearest neighbor clustering algorithm. When the energy is increased, the shape parameters are also increased because the shower is larger in all directions. The shower also is larger in all directions when increasing dlayer $=\mathrm{dU}=\mathrm{dV}$ because more hits originally outside the cluster are being included. The dependence of the shape parameters on energy and clustering inputs make these characteristics more difficult to use in identifying electromagnetic showers. However, the ratios between the different shape parameters remain fairly constant and might prove useful for identification. For example, the ratio of shape parameter two to three is still very close to one despite its slight dependence on energy and clustering algorithm inputs.

As can be seen in Figure 16, all the clustering algorithms have a constant mean number of clusters detected for opening angles greater than one degree. Since the means are all near two, all the clustering algorithms were able to resolve the two photons with these opening angles. Despite this non-dependence on opening angle, the nearest neighbor algorithm has a significantly lower mean than the other two more advanced clustering algorithms for opening angles greater than one degree. This suggests that the directed tree and fixed cone clustering algorithms are more successful at resolving two nearby photons on average for these angles. However, for an opening angle of one degree, the mean number of clusters for the nearest neighbor clustering 
algorithm is very similar to the mean number for the larger opening angles, whereas the mean number of clusters for the directed tree and fixed cone clustering algorithms decreased dramatically to a value closer to one. This suggests that for an opening angle of one degree, the direct tree and fixed cone algorithms cannot resolve the two photons.

With this project, many characteristics of a simulated EM calorimeter for the ILC were analyzed. The energy resolutions of three different calorimeter designs were compared and showed that a heterogeneous calorimeter may be a low-cost way to attain good resolution. Characteristics of EM showers were identified that can help differentiate showers in the calorimeter. The spatial resolution using three clustering algorithms was investigated, and the more advanced algorithms, directed tree and fixed cone, out-performed the nearest neighbor algorithm for opening angles greater than one degree. There is still much more research to be done, including using single particle events that hit the detector at various angles and identifying EM showers using different clustering algorithms. With enough analysis, the best possible calorimeter for the ILC can be designed to help detect new and exciting physics.

\section{ACKNOWLEDGMENTS}

This research was conducted at the Stanford Linear Accelerator Center through the Science Undergraduate Laboratory Internship program organized by the U.S. Department of Energy, Office of Science. They have my heartfelt gratitude for providing me with a wonderful learning experience. I thank Ronald Cassell for his help in many areas of my research and Jeremy McCormick for the use of his detector simulation program. Special thanks go to my mentors Norman Graf and Tony Johnson who directed my analyses with enthusiasm and patience and made this a thoroughly enjoyable experience.

\section{REFERENCES}

[1] M. Perricone, "Super-fast Super-sensitive Detectors," in Symmetry - Dimensions of Particle Physics, Vol.2, Is. 07, Sept. 2005, pp. 18-23. 
[2] SLIC documentation and tutorials can be found at: https://confluence.slac.stanford.edu/display/ilc/org.lcsim

[3] J. Allison, et al., "Geant4 Developments and Applications," in IEEE Transactions on Nuclear Science, Vol. 53, No. 1, Feb. 2006, pp. 270-278.

[4] C.W. Fabjan and T. Ludlam, "Calorimetry in High-Energy Physics," in Annual Review of Nuclear and Particle Science, Vol. 32, Dec. 1982, pp. 335-389. 


\begin{tabular}{|l|c|c|}
\hline & \multicolumn{2}{|c|}{ Absorber Thickness (cm) } \\
\hline Homogeneous 30 Layer & \multicolumn{2}{|c|}{0.271} \\
\hline Homogeneous 40 Layer & \multicolumn{2}{|c|}{0.271} \\
\hline Heterogeneous 30 Layer & 0.271 (for layers 1-20) & 0.543 (for layers 21-30) \\
\hline
\end{tabular}

Table 1. Absorber thicknesses in each layer for the different calorimeter designs.

\begin{tabular}{|l|c|c|c|}
\hline & p0 & p1 & $\boldsymbol{\chi}^{\mathbf{2}}$ \\
\hline Homogeneous 30 Layer & 0.017002 & 0.97942 & 43.086 \\
\hline Homogeneous 40 Layer & -0.0033657 & 0.99702 & 2.6931 \\
\hline Heterogeneous 30 Layer & 0.014626 & 0.98242 & 29.274 \\
\hline
\end{tabular}

Table 2. Values of the linear fits for total energy detected in the calorimeter versus known photon energy using different calorimeter designs. The fitted function has the form $\mathrm{p} 0+\mathrm{p} 1 \mathrm{x}$.

\begin{tabular}{|l|c|c|c|}
\hline & p0 & p1 & $\boldsymbol{\chi}^{\mathbf{2}}$ \\
\hline Homogeneous 30 Layer & 0.00014060 & 0.19490 & 16.792 \\
\hline Homogeneous 40 Layer & -0.0021909 & 0.19905 & 6.2012 \\
\hline Heterogeneous 30 Layer & 0.0010234 & 0.19593 & 6.4259 \\
\hline
\end{tabular}

Table 2. Values of the energy resolution linear fits using different calorimeter designs. The fitted function has the form $\mathrm{p} 0+\mathrm{p} 1 \mathrm{x}$. 


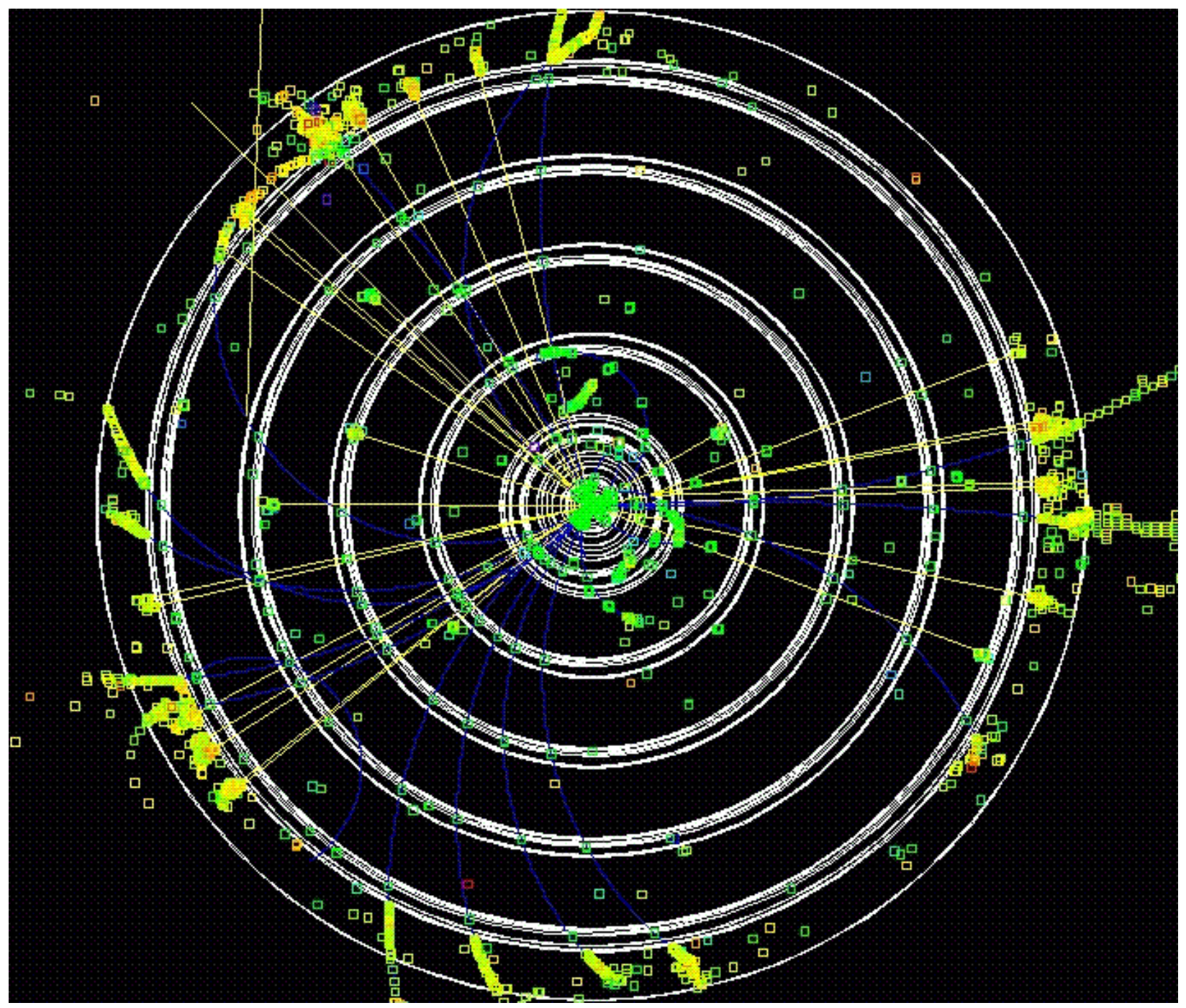

Figure 1. Example of the WIRED4 event display showing a simulated electron-positron collision with tracks of particles and hits detected in the calorimeter.

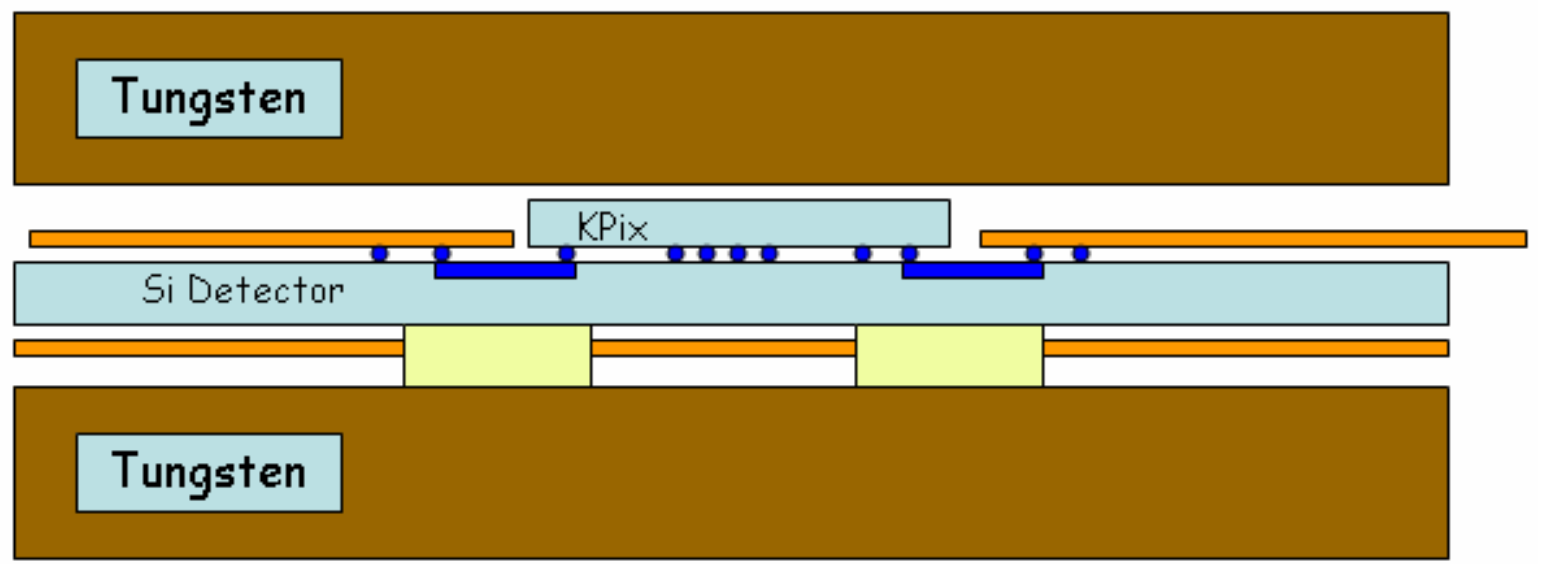

Figure 2. Diagram of a layer in the heterogeneous calorimeters being analyzed in this research. KPix is the readout chip for the detector. 


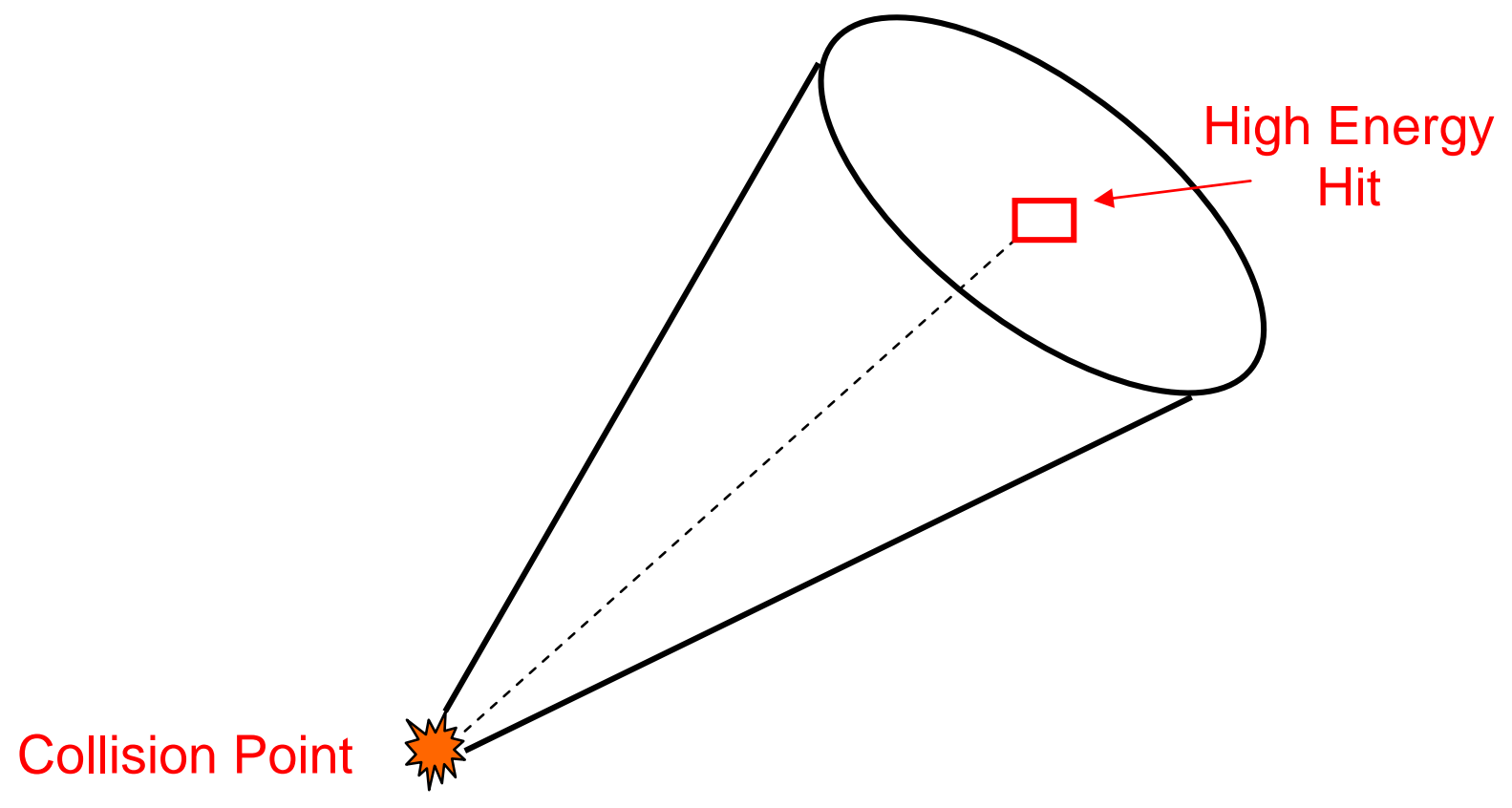

Figure 3. Diagram of the fixed cone clustering algorithm. The algorithm starts looking at the highest energy hits, then draws a cone about this hit with vertex at the collision point. All hits within the cone are grouped together into one cluster. 
Total Energy in the EM Calorimeter vs. Photon Energy with Homogeneous 30 Layer Calorimeter

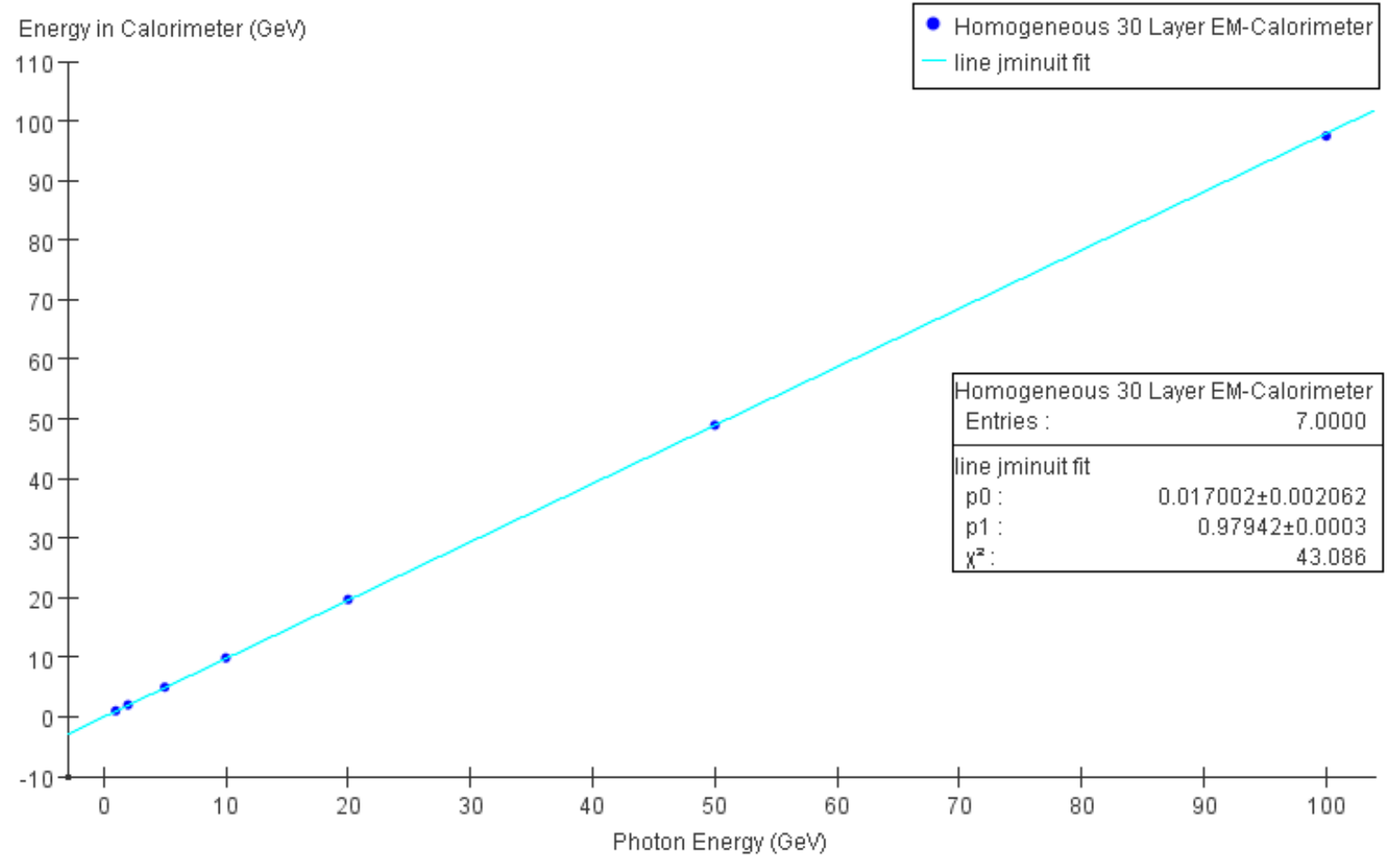

Figure 4. Linearity plot of the total energy in the homogeneous thirty layer EM calorimeter versus the actual energy of a single photon event. The data points are fitted with a linear function of the form $\mathrm{p} 0+\mathrm{p} 1 \mathrm{x}$.

Total Energy in the EM Calorimeter vs. Photon Energy with Homogeneous 40 Layer Calorimeter

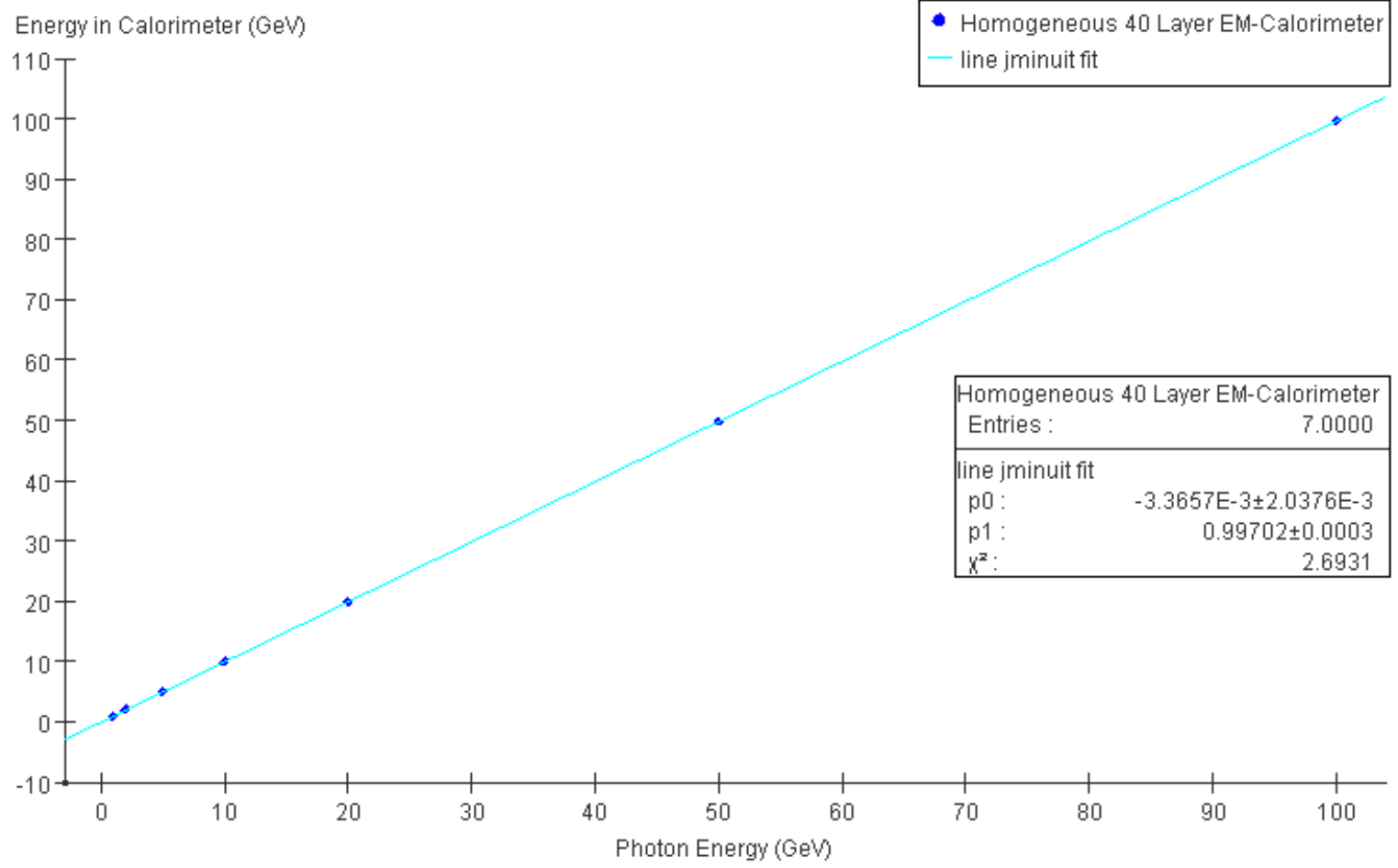

Figure 5. Linearity plot of the total energy in the homogeneous forty layer EM calorimeter versus the actual energy of a single photon event. The data points are fitted with a linear function of the form $\mathrm{p} 0+\mathrm{p} 1 \mathrm{x}$. 
Total Energy in the EM Calorimeter vs. Photon Energy with Heterogeneous 30 Layer Calorimeter

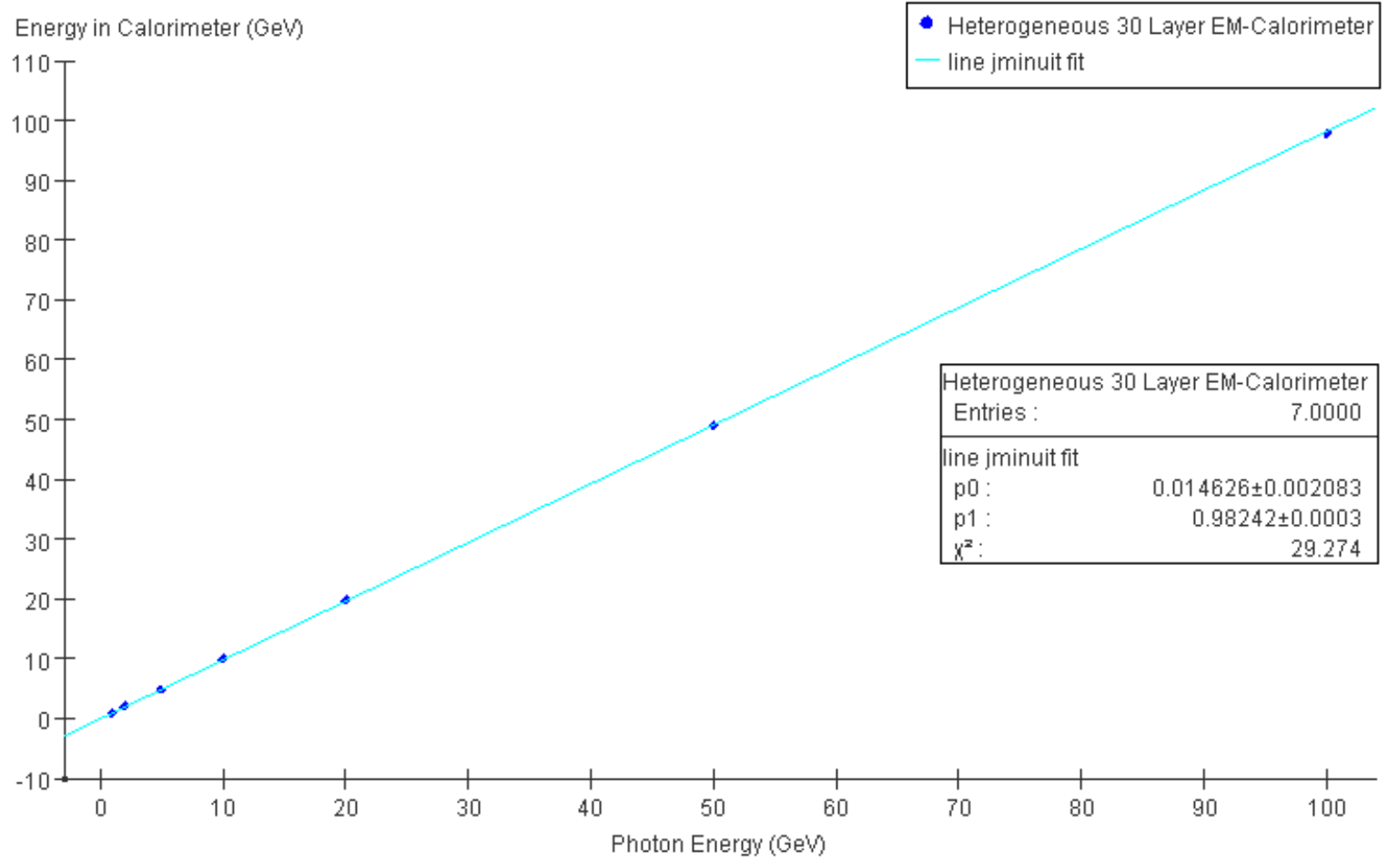

Figure 6. Linearity plot of the total energy in the heterogeneous forty layer EM calorimeter versus the actual energy of a single photon event. The data points are fitted with a linear function of the form $\mathrm{p} 0+\mathrm{p} 1 \mathrm{x}$.

\section{Total Cluster Energy vs. dU or dV}

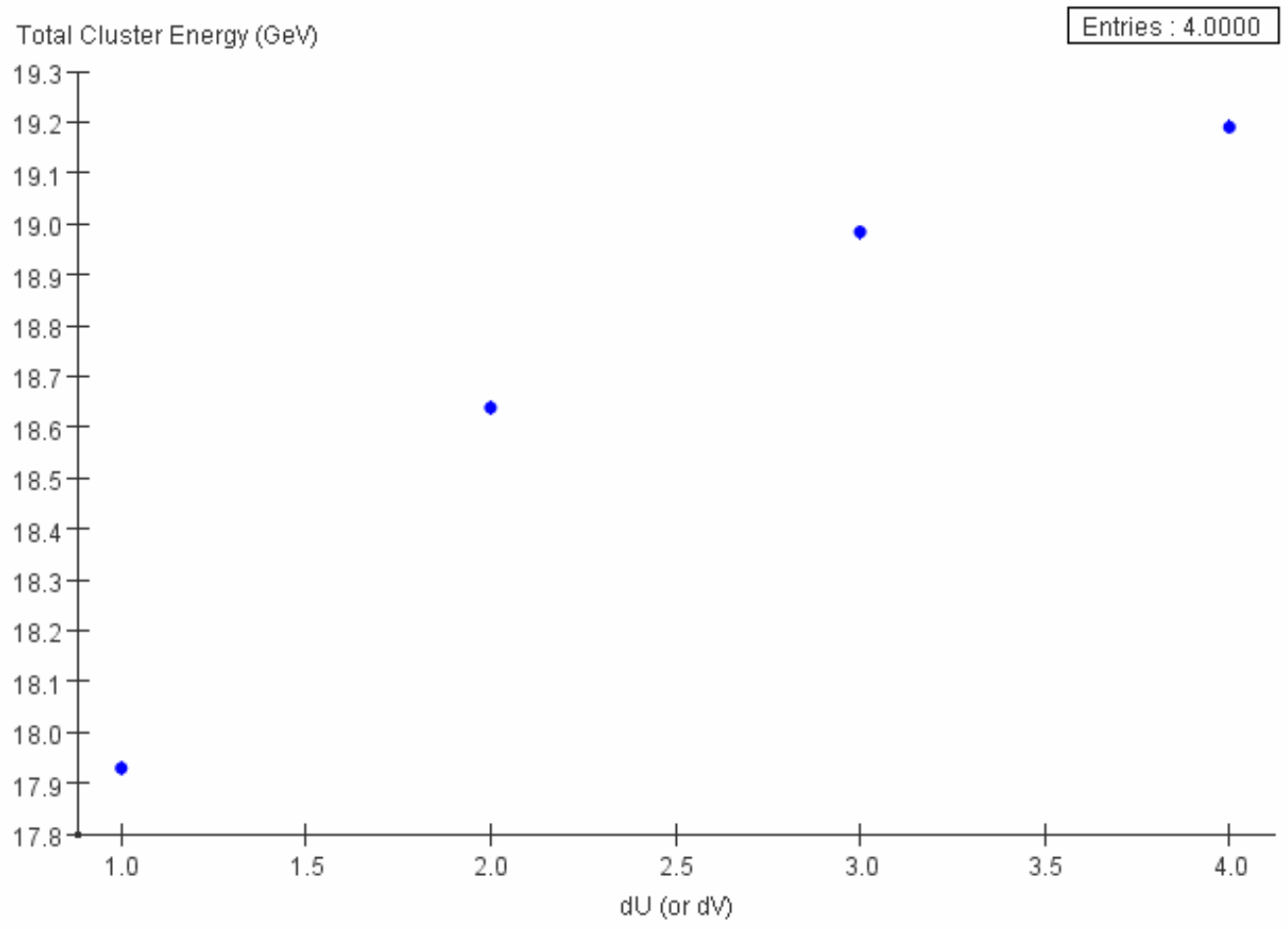

Figure 7. Plot of the total cluster energy for a $20 \mathrm{GeV}$ photon using the nearest neighbor clustering algorithm and varying input parameters $\mathrm{dU}=\mathrm{dV}$. 


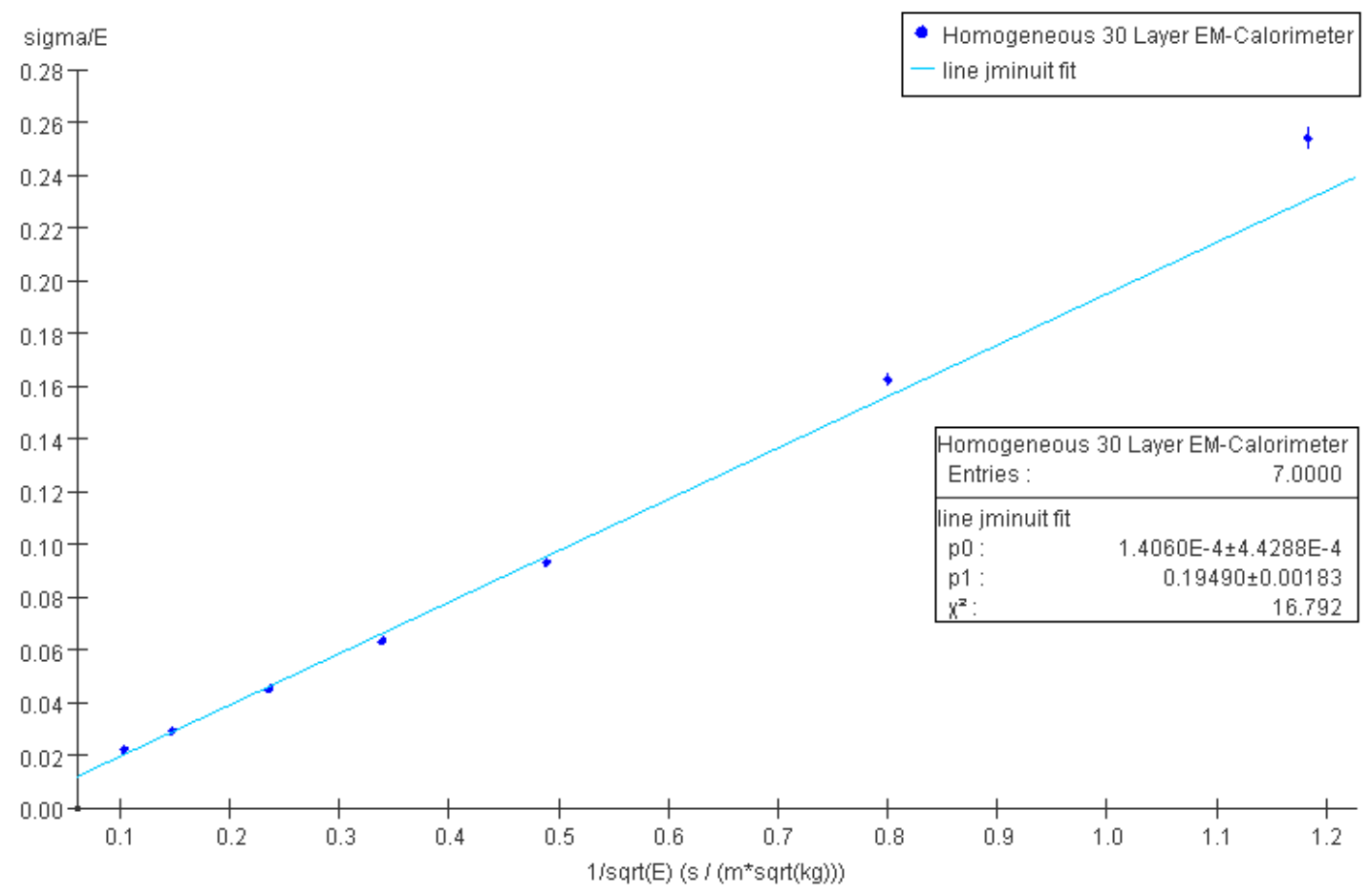

Figure 8. Energy resolution for single photons of varying energy using the homogeneous thirty layer EM calorimeter. The data points are fitted with a linear function of the form $\mathrm{p} 0+\mathrm{p} 1 \mathrm{x}$.

Energy Resolution with Homogeneous $\mathbf{4 0}$ Layer Calorimeter

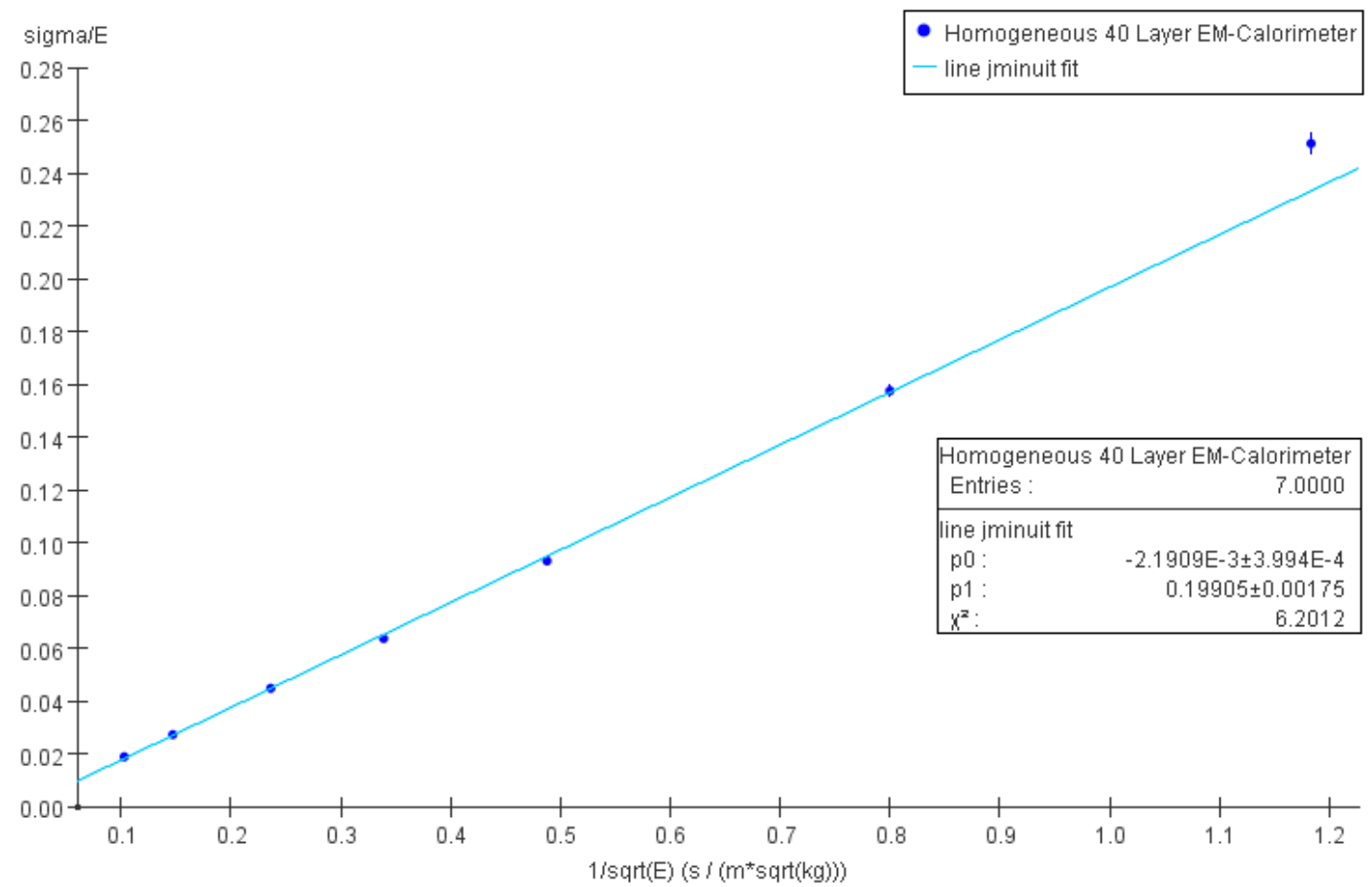

Figure 9. Energy resolution for single photons of varying energy using the homogeneous forty layer EM calorimeter. The data points are fitted with a linear function of the form $\mathrm{p} 0+\mathrm{p} 1 \mathrm{x}$. 
Energy Resolution with Heterogeneous 30 Layer Calorimeter

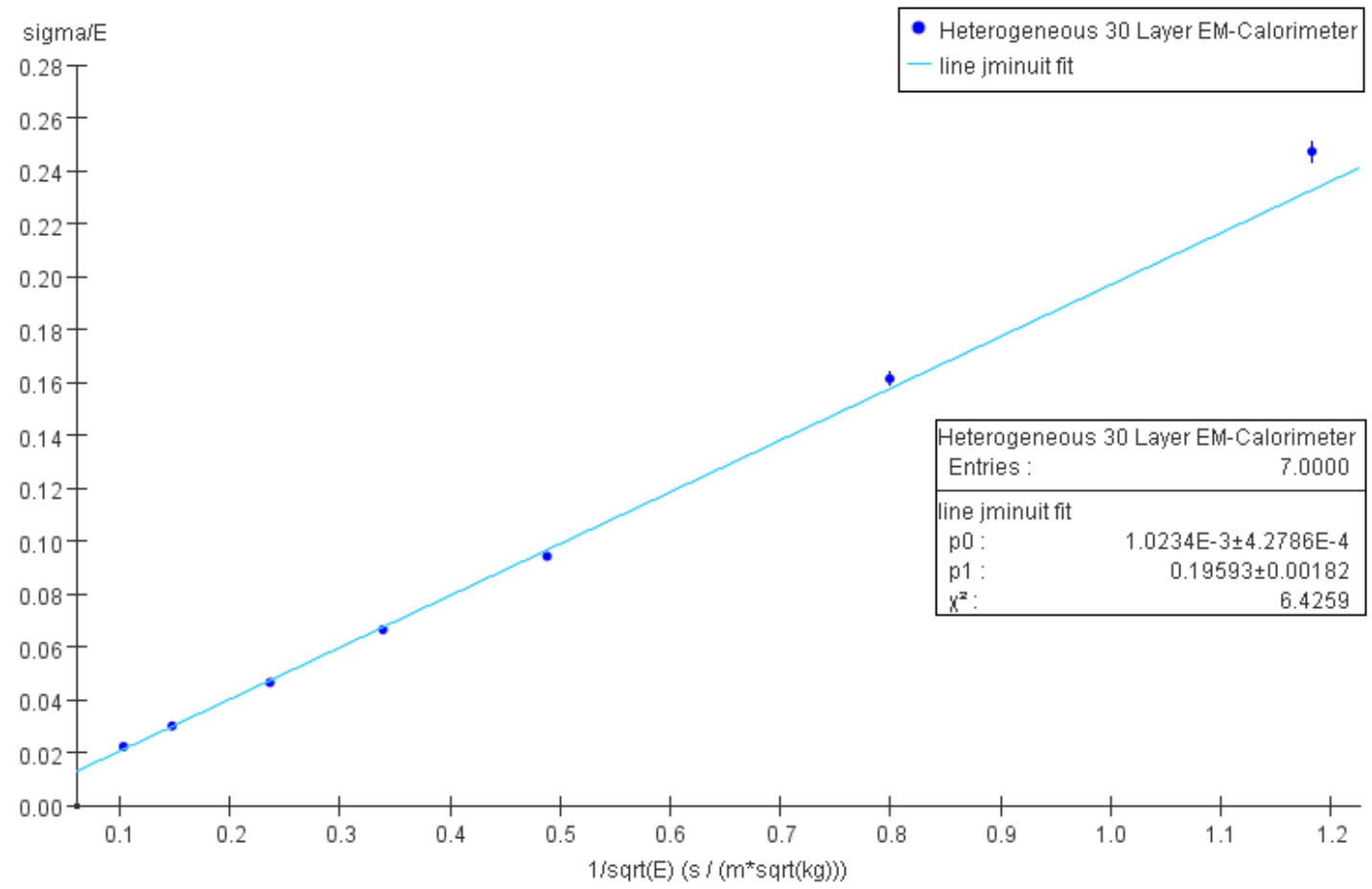

Figure 10. Energy resolution for single photons of varying energy using the heterogeneous thirty layer EM calorimeter. The data points are fitted with a linear function of the form $\mathrm{p} 0+\mathrm{p} 1 \mathrm{x}$. 


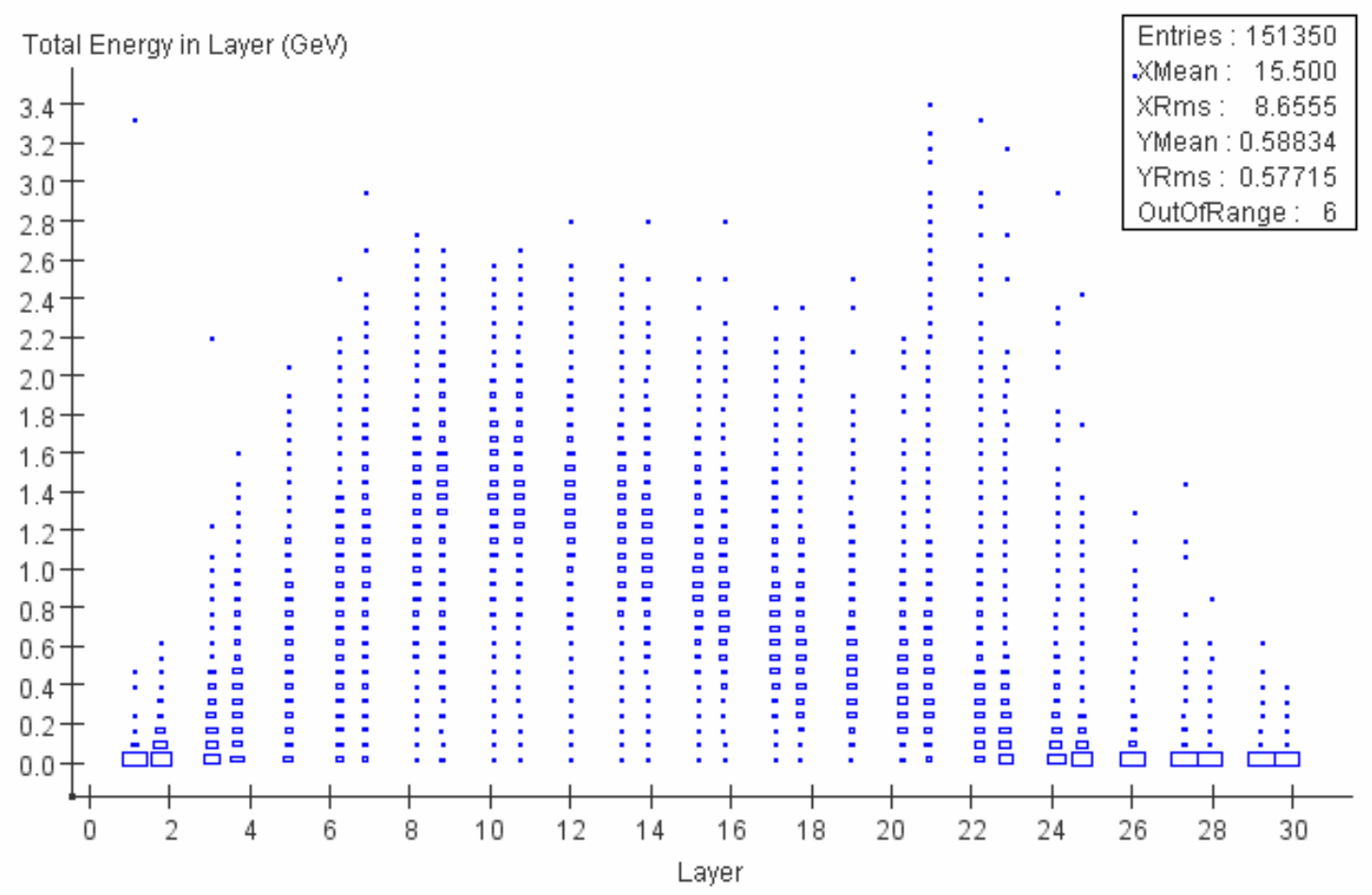

Figure 11. The total energy in each layer of the electromagnetic calorimeter for single $20 \mathrm{GeV}$ photons.

Width vs. Layer

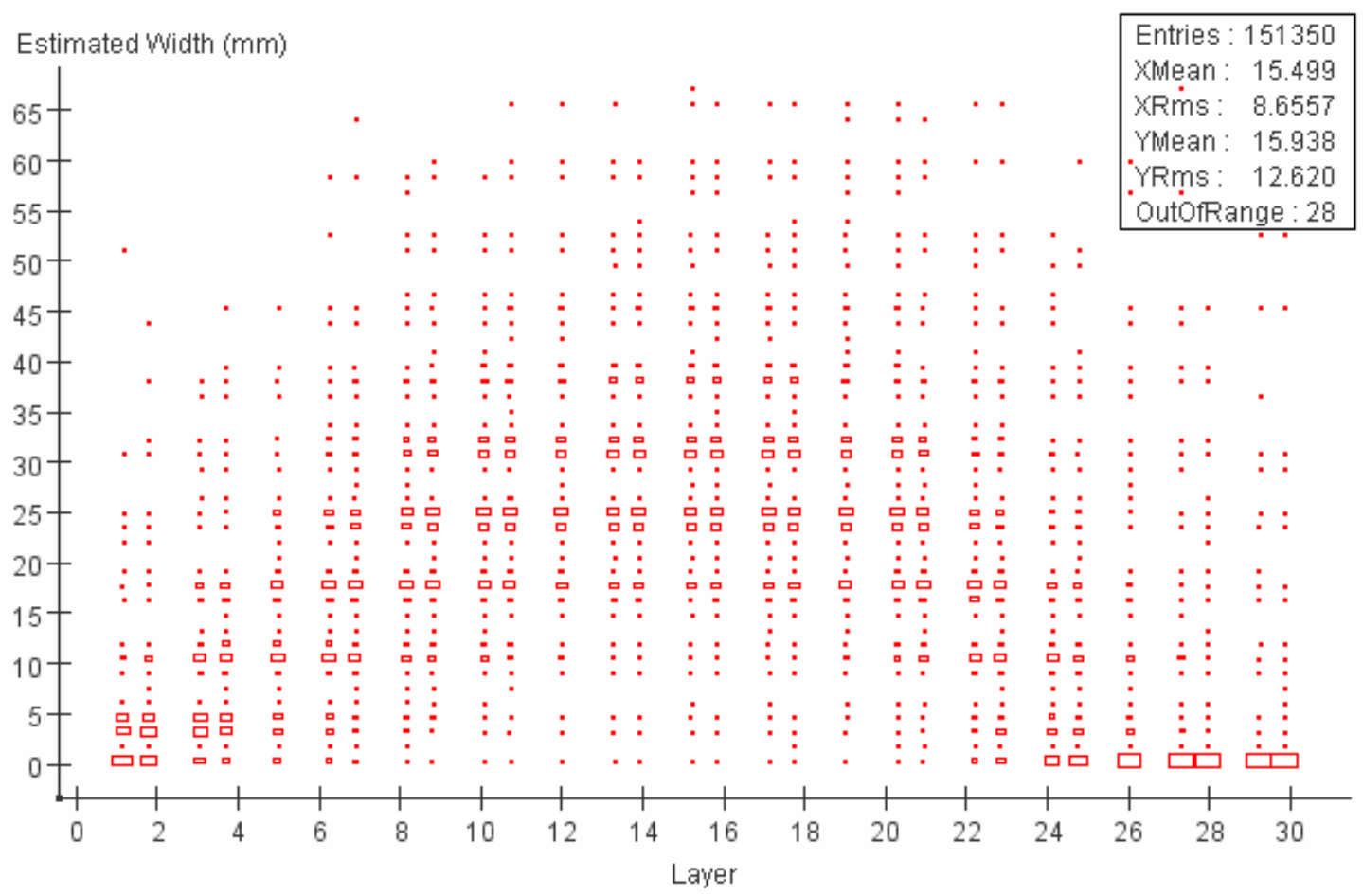

Figure 12. The approximate width of the cluster in each layer of the electromagnetic calorimeter for single $20 \mathrm{GeV}$ photons. 


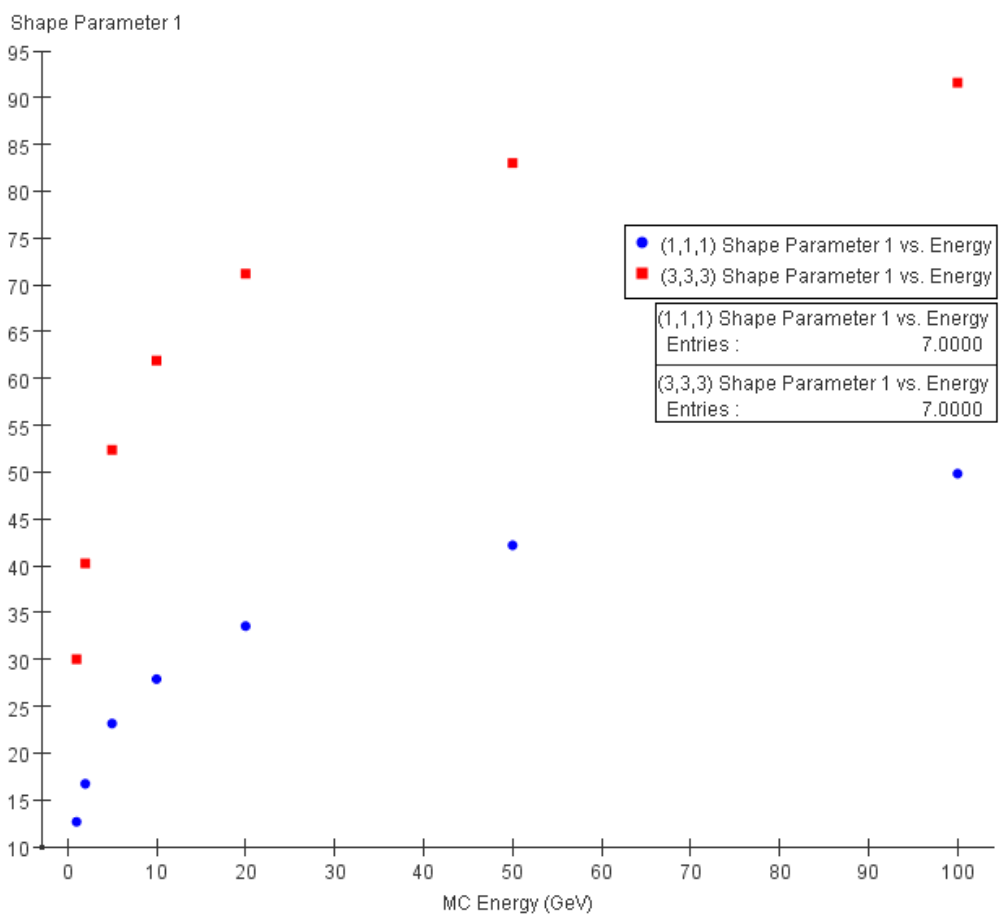

Figure 13. First shape parameter of a single photon cluster versus photon energy with $d U=d V=d l a y e r=1$, labeled $(1,1,1)$ Shape Parameter 1 in the figure, and $d U=d V=$ dlayer $=3$, labeled $(3,3,3)$ Shape Parameter 1 in the figure.

Shape Parameter 2 vs. Energy

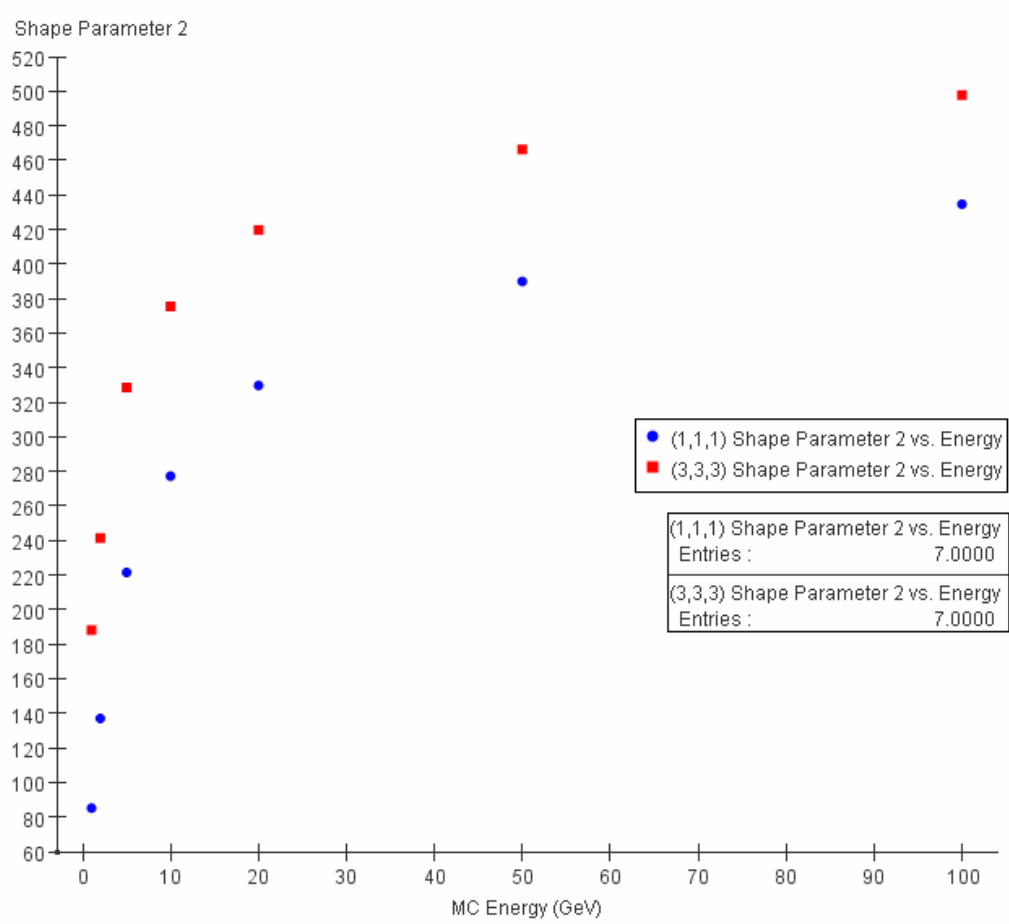

Figure 14. Second shape parameter of a single photon cluster versus photon energy with $d U=d V=d l a y e r=1$, labeled $(1,1,1)$ Shape Parameter 2 in the figure, and $d U=d V=$ dlayer $=3$, labeled $(3,3,3)$ Shape Parameter 2 in the figure. 


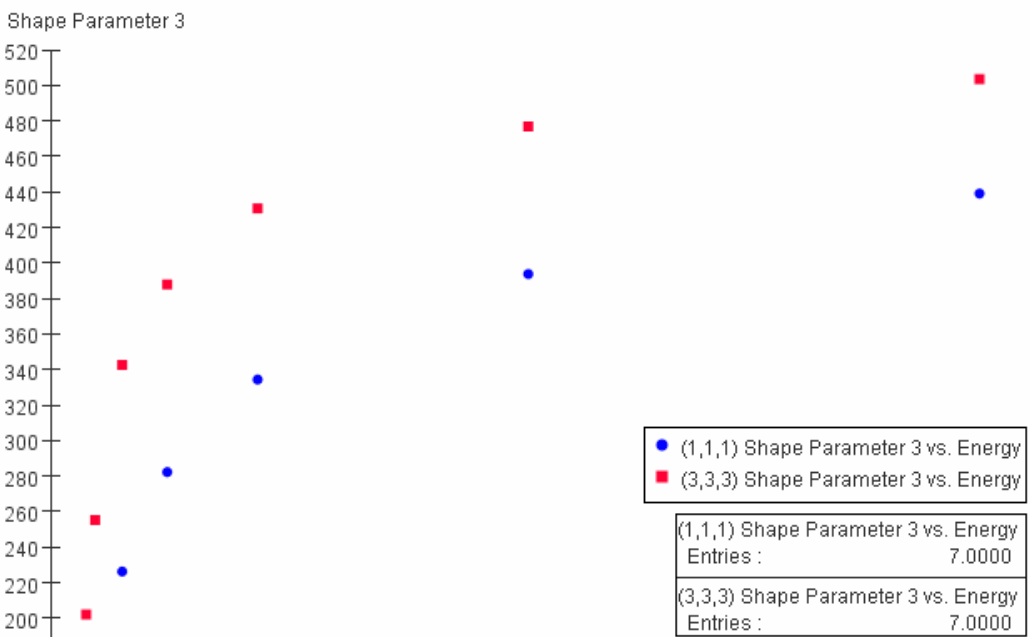

Figure 15. Third shape parameter of a single photon cluster versus photon energy with $d U=d V=$ dlayer $=1$, labeled $(1,1,1)$ Shape Parameter 3 in the figure, and $d U=d V=$ dlayer $=3$, labeled $(3,3,3)$ Shape Parameter 3 in the figure.

Mean Number of Clusters vs. Opening Angle for Two 1GeV Photons

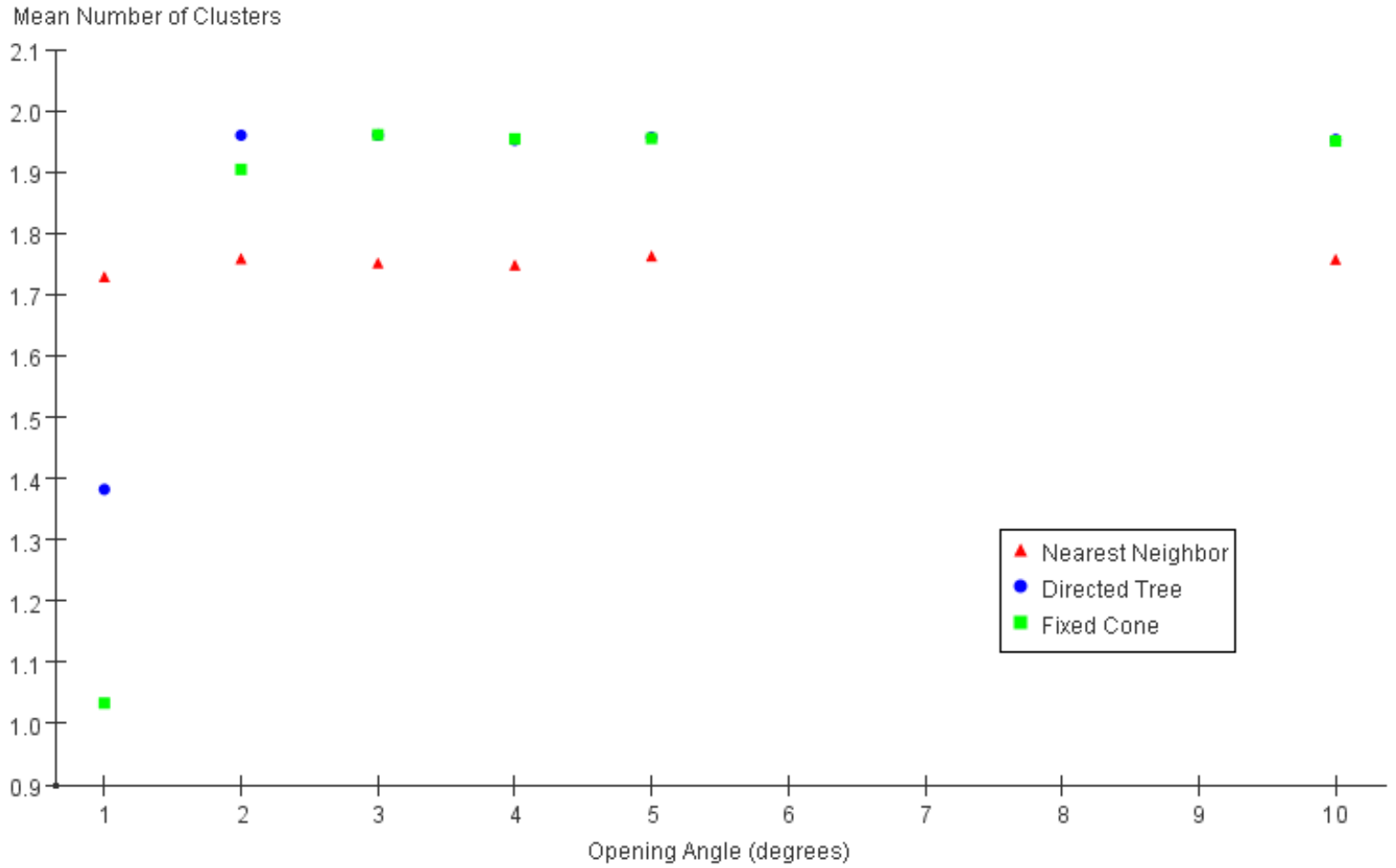

Figure 16. The mean number of clusters detected for two $1 \mathrm{GeV}$ photons with various opening angles using the clustering algorithms: nearest neighbor, directed tree, and fixed cone. 


\title{
Simulations of the ILC Electron Gun and Electron Bunching System
}

\author{
Christian Bernt Haakonsen \\ Office of Science, SULI Program \\ McGill University \\ Stanford Linear Accelerator Center \\ Menlo Park, CA
}

August 25, 2006

Prepared in partial fulfillment of the requirements of the Office of Science, U.S. Department of Energy Science Undergraduate Laboratory Internship (SULI) Program under the direction of Axel Brachmann at the Stanford Linear Accelerator Center.

Participant:

Signature

Research Advisor:

Signature 


\section{Table of Contents}

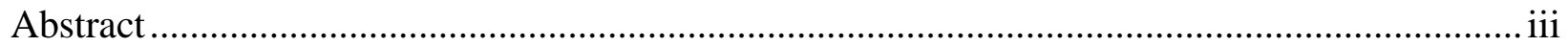

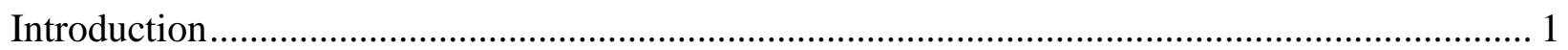

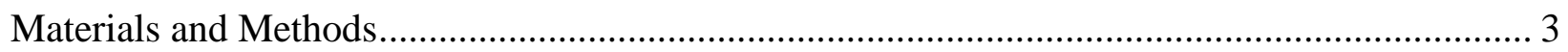

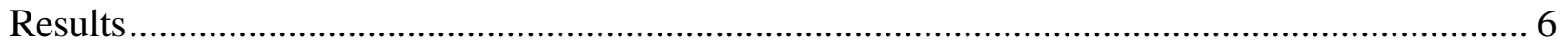

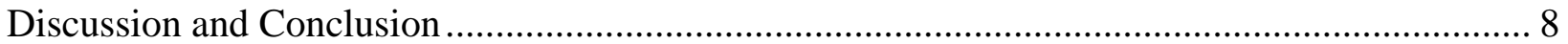

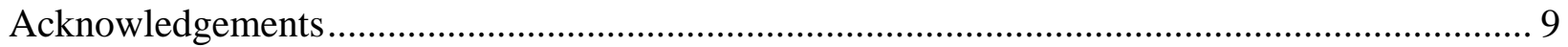

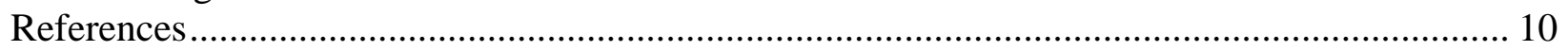

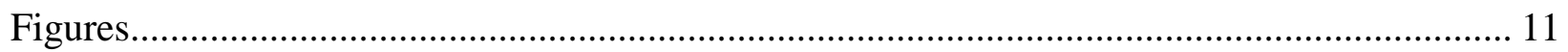

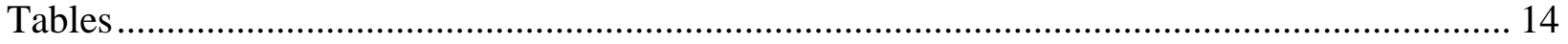




\begin{abstract}
Simulations of the ILC Electron Gun and Electron Bunching System. CHRISTIAN BERNT HAAKONSEN (McGill University, Montreal, Quebec, CANADA) AXEL BRACHMANN (Stanford Linear Accelerator Center, Menlo Park, CA).
\end{abstract}

The International Linear Collider (ILC) is a proposed electron-positron collider, expected to provide insight into important questions in particle physics. A part of the global R\&D effort for the ILC is the design of its electron gun and electron bunching system. The present design of the bunching system has two sub-harmonic bunchers, one operating at $108 \mathrm{MHz}$ and one at 433MHz, and two 5-cell 1.3 GHz (L-band) bunchers. This bunching system has previously been simulated using the Phase and Radial Motion in Electron Linear Accelerators (PARMELA) software, and those simulations indicated that the design provides sufficient bunching and acceleration. Due to the complicated dynamics governing the electrons in the bunching system we decided to verify and expand the PARMELA results using the more recent and independent simulation software General Particle Tracer (GPT). GPT tracks the motion and interactions of a set of macro particles, each of which represent a number of electrons, and provides a variety of analysis capabilities. To provide initial conditions for the macro particles, a method was developed for deriving the initial conditions from detailed simulations of particle trajectories in the electron gun. These simulations were performed using the Egun software. For realistic simulation of the L-band bunching cavities, their electric and magnetic fields were calculated using the Superfish software and imported into GPT. The GPT simulations arrived at similar results to the PARMELA simulations for sub-harmonic bunching. However, using GPT it was impossible to achieve an efficient bunching performance of the first L-band bunching cavity. To 
correct this, the first L-band buncher cell was decoupled from the remaining 4 cells and driven as an independent cavity. Using this modification we attained results similar to the PARMELA simulations. Although the modified bunching system design performed as required, the modifications are technically challenging to implement. Further work is needed to optimize the L-Band buncher design. 


\section{Introduction}

To answer fundamental questions that have arisen in particle physics, physicists are planning the International Linear Collider (ILC). The ILC will be a $1 \mathrm{TeV}$ electron-positron collider, designed to probe the same energy scale as the nearly completed Large Hadron Collider (LHC). Compared to the proton-antiproton collisions of the LHC, the ILC's electron-positron collisions will provide a better signal to noise ratio, no bias towards producing strongly interacting particles, and a well known center of mass frame for each elementary particle collision. This will allow the ILC to study the properties of the particles discovered at the LHC, and discern different theories that predict similar results at the LHC.

A part of the global R\&D effort for the ILC is the design of its electron gun and electron bunching system. These are currently being developed at the Stanford Linear Accelerator Center, and are the focus of this paper. The electron gun generates electrons (3.2 nC per bunch) through the illumination of a photocathode by a 2 ns laser pulse. A cathode bias of $-120 \mathrm{keV}$ accelerates the electrons to $\beta=V / c=0.59$ at the gun exit. Since the electron bunch extends more than a full wavelength of the $1.3 \mathrm{GHz}$ (L-band) driving frequency of the main ILC linac, sub-harmonic bunching is required for the initial shortening of the bunch, before L-band bunchers compress and accelerate to produce a $20 \mathrm{ps}, 10 \mathrm{MeV}$ electron bunch .

The bunching system outlined in [1] for the TESLA accelerator is used as the preliminary ILC bunching design. In this design a first sub-harmonic buncher (SHB1) is operated at 108 $\mathrm{MHz}$, and a second at $433 \mathrm{MHz}$ (SHB2) at a distance of $2.02 \mathrm{~m}$. The first of two L-band bunchers is located $0.33 \mathrm{~m}$ from the second subharmonic buncher, with the second L-band buncher spaced $0.1 \mathrm{~m}$ from the first. Each L-band buncher is a 5-cell RF standing wave cavity, and both share a single klystron. 
Computer simulations are an important tool in testing and improving electron gun and bunching designs. In the electron gun a solution to Poisson's equation is needed to calculate the electron trajectories. Also, Child's law gives the space-charge limited current which cannot be exceeded [2: 46]. Iteratively tracing rays of current and numerically solving Poisson's equation leads to a convergent solution for the currents in the gun. At the exit of the gun it must be evaluated if space-charge effects are significant in the dynamics of the electron bunch. An estimate of the ratio of space-charge to emittance effects on the bunch envelope evolution is given by:

$$
R_{0}=\frac{2 I \sigma^{2}}{I_{0} \gamma^{2} \varepsilon^{2}}
$$

where I is the peak current, $\sigma$ the bunch radius, $\gamma$ the relativistic parameter, $\varepsilon$ the unnormalized emittance, and the characteristic electron current $I_{0}=\frac{e \cdot c}{r_{e}}$ (where $\mathrm{r}_{\mathrm{e}}$ is the radius of an electron) [3]. At $120 \mathrm{keV}$ and the order of magnitude of emittance we expect at the gun exit $\mathrm{R}_{0}$ will be larger than 1, which indicates that space-charge effects must be considered. For a much larger energy or emittance space-charge could be ignored and $R_{0}<<1$. The presence of space-charge effects makes this a many body problem, and it can usually be solved by grouping electrons into macro particles and calculating the motion of the macro particles numerically. If convergence to a solution occurs as the number of macro particles is increased (thus decreasing the number of electrons per macro particle) it is unnecessary to simulate individual electron interactions (which would take prohibitively long on current hardware).

This paper presents the results of simulations of the electron gun and bunching section of the ILC, and compares them to the simulations presented in [1]. Several software tools are used 
in conjunction to simulate the electron bunch propagation as accurately as possible, and areas requiring further study are proposed.

\section{Materials and Methods}

Simulations were preformed on a PC with an Intel Pentium 42.4 GHz CPU and 2 GB of RAM.

The electron gun was simulated using the Egun version 2 (v2) software by entering an r-z cylindrically symmetric gun design, the radius of the emitting area of the cathode, and the cathode bias voltage. In addition the perveance, $\mathrm{K}$, was specified to limit the current to the intended $1.6 \mathrm{~A}$, which in the actual gun will be determined by the laser intensity [4: 36]. Perveance, bias voltage and current are related by:

$$
K=\frac{I}{\sqrt{V^{3}}},
$$

Current and operating voltage result in a perveance of $0.03849 \mu$ perv for this gun. Egun calculates current rays representing the electron trajectories, and calculates each ray's radial position, total current, kinetic energy and angular divergence at the end of the gun. Particle trajectories are used to determine the unnormalized (3) and normalized edge emittance (4) of the beam at the gun exit [2: 57]:

$$
\begin{aligned}
& \varepsilon_{\text {edge }}=4 \cdot \sqrt{\overline{x^{2}} \cdot \overline{x^{\prime 2}}-\overline{x^{2} \cdot x^{\prime 2}}} \\
& \varepsilon_{\text {edge }, N}=\beta \cdot \gamma \cdot \varepsilon_{\text {edge }} .
\end{aligned}
$$

To simulate the bunching system we used the General Particle Tracer v2.71 (GPT) software. Its inputs are a list of fields and elements that act on the electrons, and the initial conditions of the macro particles representing the electrons. The GPT Multiple Run (MR) feature 
allows running the simulation for a several dimensional parameter space. This technique was used to optimize a set of parameters such as field amplitudes, distances between elements, and initial phase of oscillating electromagnetic fields. To increase simulation speed the radial dependence of the axial solenoidal magnetic fields were ignored. This is justified as the electrons propagate near the axis of the solenoidal fields at all times.

The Egun final ray states were used to calculate the initial conditions for the macro particles in GPT. Each ray was taken to represent a number of electrons (GPT input parameter nmacro) corresponding to the ray current. Due to the cylindrical symmetry of the gun, Egun does not give a cylindrical angle in the plane perpendicular to the axis, only a radius. To spread the macro particles from a radial line to a disk we assigned each particle a random angle, $\theta$, in the interval $(0 . .2 \pi)$ to calculate the $\mathrm{x}$ - and $\mathrm{y}$-components of its position. A scaling from $\mathrm{mm}$ to $\mathrm{m}$ was also preformed. The kinetic energy $(T)$, rest mass energy $\left(E_{0}\right)$ and the angular divergence $(\alpha)$ were used with $\theta$ to calculate the following GPT input parameters:

$$
\begin{aligned}
& \gamma \beta_{x}=\left(1+\frac{T}{E_{0}}\right) \sqrt{1-\left(1+\frac{T}{E_{0}}\right)^{-2}} \sin (\alpha) \cos (\theta), \\
& \gamma \beta_{y}=\left(1+\frac{T}{E_{0}}\right) \sqrt{1-\left(1+\frac{T}{E_{0}}\right)^{-2}} \sin (\alpha) \sin (\theta), \\
& \gamma \beta_{z}=\left(1+\frac{T}{E_{0}}\right) \sqrt{1-\left(1+\frac{T}{E_{0}}\right)^{-2}} \cos (\alpha) .
\end{aligned}
$$

These are required by GPT for each macro particle in addition to the three spatial coordinates and number of electrons. The macro particles are started by GPT uniformly as a function of time over 2 ns to closely resemble the actual particle distribution at the gun exit. Because Egun lists 
the rays in order of increasing starting radius at the cathode, the order of the macro particles was randomized before input to GPT to avoid correlation between starting time and initial radius.

To calculate the L-band buncher fields using the Superfish v7 software, two types of cells were considered, central and end cells. The cells are all cylindrically symmetric and specified to Superfish as r-z plane geometry. The central cell is symmetric about its z midpoint, with an RF source on the cell wall at the plane of symmetry. The cell wall was defined as a Dirichlet boundary, whereas the iris planes on both ends were defined as Neumann boundaries. The end cell was extended on one side to account for fringe fields at the end of each cavity. The output from Superfish was an $\mathrm{r}-\mathrm{z}$ grid specifying $\mathrm{E}_{\mathrm{z}}, \mathrm{E}_{\mathrm{r}},|E|$, and $\mathrm{H}$, at each point. The lengths were scaled from cm to m, E components from $\mathrm{MV} / \mathrm{m}$ to $\mathrm{V} / \mathrm{m}$, and $\mathrm{H}$ from $\mathrm{A} / \mathrm{m}$ to $\mathrm{T}$ (factor of $\mu_{0}$ ), before conversion to the GPT data format. To obtain the end cell field at the opposite side of the cavity, the z-coordinates of the original end cell field map were reversed, and the sign of the $\mathrm{E}_{\mathrm{r}}$ components changed to preserve right-handedness.

In addition to the built in functions of 'gdfa', GPT's analysis tool, three custom functions were written to analyze the simulation output. The first was zdist, which calculates the distance between the first and last electron in the bunch. The second was tlen, which calculates the time between the first and last electron passing a point in space. The final custom function was nelectrons, which counts the number of electrons remaining in the bunch, thereby accounting for lost particles. 


\section{Results}

Egun simulations were performed to calculate the emittance as function of laser spot radius on the cathode (Fig. 1). A global minimum was found for a laser radius of $3.6 \mathrm{~mm}$, and local minima for $2.5 \mathrm{~mm}$ and $4.6 \mathrm{~mm}$. The data points below a radius of $2 \mathrm{~mm}$ are inconsistent with Child's law, as too much current is being forced from a small area, and are therefore not further considered. To investigate detail of the three local minima the simulations were repeated while forcing a current of $2.4 \mathrm{~A}$, and the local minima were found to be at the same radii as for 1.6 A. It was found that the positions of the local minima are independent of current, and that they modulate a large overall $r$ dependence, which is current dependent. The gun geometry with equipotential lines and current rays for a laser radius of $3.6 \mathrm{~mm}$ is shown in Fig. 2.

A combination of parameters that would replicate the bunching performance achieved in [1] could not be found. Simulations of a single 5 cell L-band buncher using various input bunch lengths, field strengths and initial phases, failed to produce a single scenario where bunching and acceleration occurred. To achieve the desired bunching and acceleration the phase of the first cell of the first buncher had to be decoupled from the remaining four cells. This is not physically possible without driving the decoupled cell separately from the others, so the second cell of the buncher was changed to an end cell to create a 4-cell buncher that could be separated as a whole from the first single L-band cavity. The fields of the first cell were modified to make a standalone cell by mirroring the field at the center plane of the cavity and changing the sign of the $\mathrm{E}_{\mathrm{r}}$ component on the mirrored side to preserve right-handedness. Thus a cell with fringe fields on both sides was produced. The axial solenoidal field was also strengthened to various degrees to 
improve electron bunch containment. With these alterations the L-band bunchers were made to function properly.

To be able to propagate the laser beam perpendicular to the photocathode plane, a bending magnet was introduced before the first sub-harmonic buncher. The angle was chosen to be $45^{\circ}$, and the recently available GPT element sectormagnet was downloaded and installed to be used as a bending magnet. To improve electron containment by the solenoidal field between the gun and the bending magnet the distance was increased from $0.75 \mathrm{~m}$ to $1.4 \mathrm{~m}$. Furthermore, the single artificial solenoidal field was replaced by a Superfish simulation of two Helmholtz coils with one focusing solenoid before and two focusing solenoids after it. The axial magnetic field before the bending magnet is shown in Fig. 3, and the field after the bending magnet (generated using GPT solenoid elements) is shown in Fig. 4. The z-axis in Fig. 3 is located at an angle of $45^{\circ}$ with respect to the z-axis in Fig. 4.

Table 1 summarizes what was found to be the optimum operating parameters for the bunching system. For these parameters a bunch length of $\sim 20$ ps (FWHM) was observed, with a particle loss of a few percent. A particle was considered lost when its distance from the average z-position exceeded five standard deviations. The final bunch length was found to be very sensitive to the phase of the single L-band cell.

The beam waist through the length of the bunching section is shown in Fig. 5. The full bunch length (zdist) and standard deviation of z (stdz) along the same length is shown in Fig. 6. In Fig. 5 and 6 the particle distribution is plotted with respect to the z-axis. The bending magnet is located at $z=0$. The section $z<0$ is the projection onto the $z$-axis without consideration of the bending angle, causing a $\frac{1}{\sqrt{2}}$ scaling of the z-coordinates. 


\section{Discussion and Conclusion}

By reduction of the laser spot radius from $10 \mathrm{~mm}$ to $3.6 \mathrm{~mm}$ the edge emittance at the end of the gun was reduced by more than an order of magnitude. However, the beam envelope is strongly influenced by space-charge effects at the gun exit ( $R_{0}=4.6$; formula (1)). It may be

possible to find a laser radius that will result in lower collimation at the end of the gun, yet provided a better contained beam waist leading up to the bending magnet due to reduced spacecharge effects. An optimized laser radius at the cathode can provide a possibility for improved bunch propagation compared to the one used in this study. Further, optimized electron gun parameters may also reduce the necessary solenoidal field for beam containment.

The L-band buncher cavities are standing wave structures designed for particles with $\beta \approx 1$. For such a design the field from one cell to another advances one half period during the time it takes particles to move one half wavelength of the wave (one cell length), and thus the particles arrive at the same phase of the wave each time it reaches maximum amplitude. This is not the case for the low energy particles generated by a $120 \mathrm{kV}$ electron gun $(\beta=0.59)$. The field in the first cell will reach a maximum before the particles have traveled a quarter wavelength.

The simulations in this study showed it is impossible to bunch a non-relativistic beam using a $\beta=1$ structure without significant particle losses. The energy transfer of such a system will lead to acceleration and decceleration of leading and trailing parts of the bunch resulting in increased energy spread without a bunching effect. To achieve bunch compression, it was necessary to decouple the phase of the first cell to ensure overlap of bunch arrival time and appropriate phase of the following L-band structure. However, for a realistic bunching system phase decoupling is impossible without spacing the first cell from the remaining four. Therefore, 
the first L-band buncher cell was separated and operated as an independent bunching cavity. The bunch propagation was found to be extremely sensitive to the initial phase of the single L-band cell compared to the other RF structures of the system. This may be problematic for a real design. Improvements of the present solution are possible. In particular a low $\beta$ L-band bunching structure should be considered.

The Parmela simulations outlined in [1] using a $\beta=1$ L-band buncher should be further reviewed to analyze details of this design. We emphasize that the fields of standing wave cavities used in the GPT simulation are generated by a Superfish model and accounts for the radial electric field components, whereas the previous PARMELA simulations used a simplified model with only axial fields. This may be a contribution to the different performance of the bunching system in the GPT and PARMELA simulations.

\section{Acknowledgements}

The research described took place at the Stanford Linear Accelerator Center, and I would like to thank my mentor, Axel Brachmann, for patient guidance as I familiarized myself with a new subject area, and through the process of producing a scientific paper. I would also like to thank Adam Edwards for several early edits and advice on improving my scientific writing.

The United States Department of Energy, Office of Science has my gratitude for organizing and funding the SULI program, without which this work would not have been possible. 


\section{References}

[1] A. Curtoni and M. Jablonca; Study of the TESLA preaccelerator for the polarised electron beam. TESLA 2001-22(2)

[2] M. Reisner, Theory and Design of Charged Particle Beams. New York: John Wiley and Sons, Inc., 1994

[3] S.G Anderson, et al., "Space-charge effects in high brightness electron beam emittance measurements," Physical Review Special Topics - Accelerators and Beams., vol. 5, 014201, Jan. 2002

[4] W. B. Herremannsfeldt, Egun - an Electron Optics and Gun Design Program. Stanford Linear Accelerator Center, Stanford University, Stanford, CA. SLAC - 331, Oct. 1988 


\section{Figures}

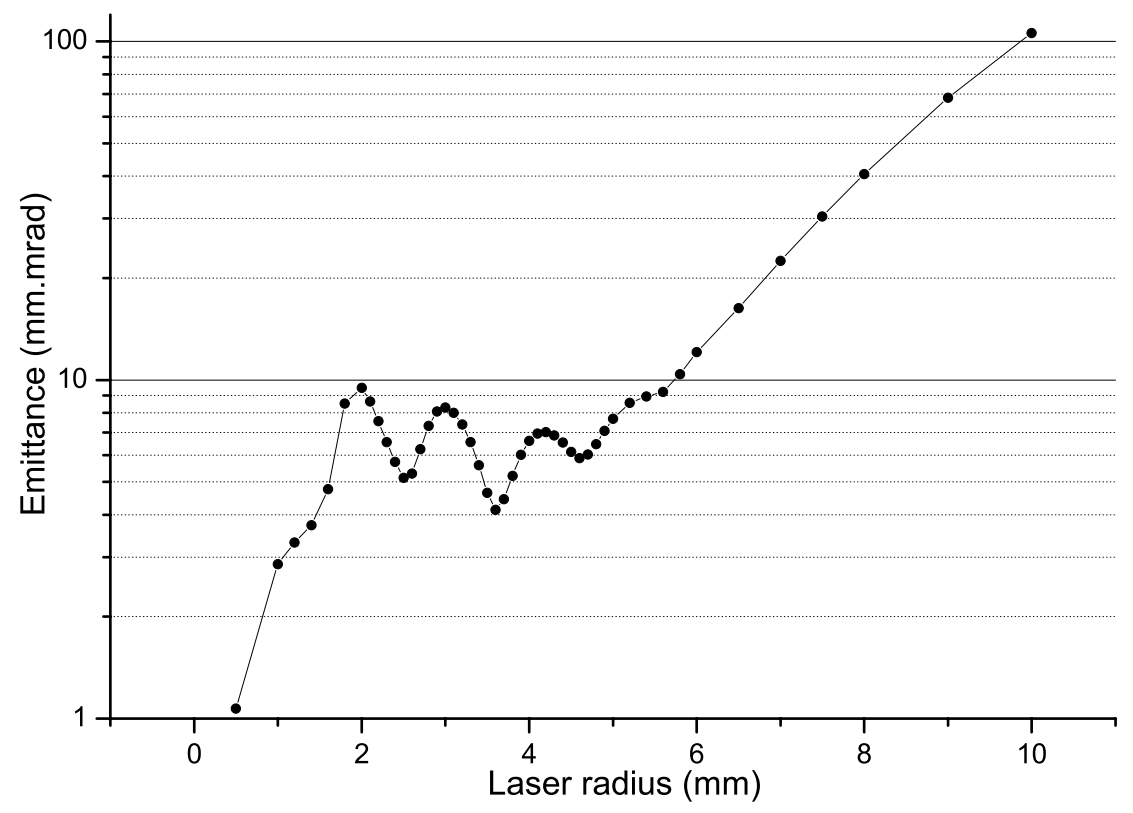

Fig. 1: Emittance of current rays at end of gun as a function of laser spot radius at photocathode

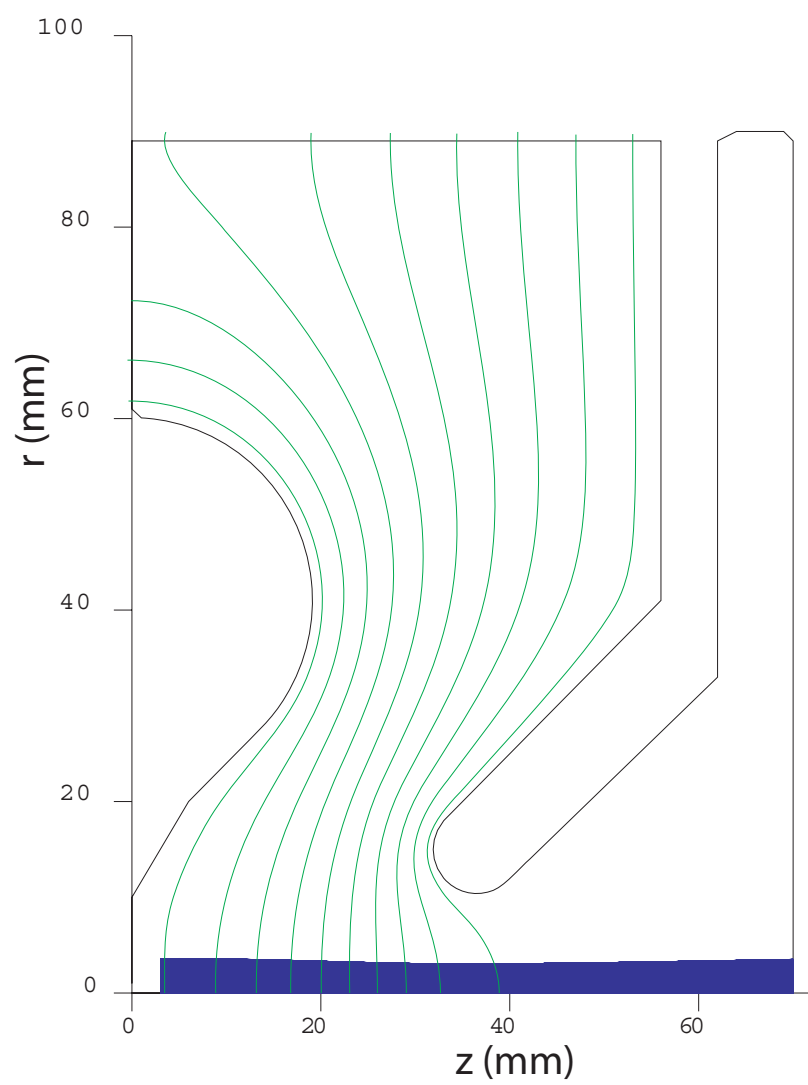

Fig. 2: Equipotential lines (green), geometry (black) and current rays (blue) in electron gun 


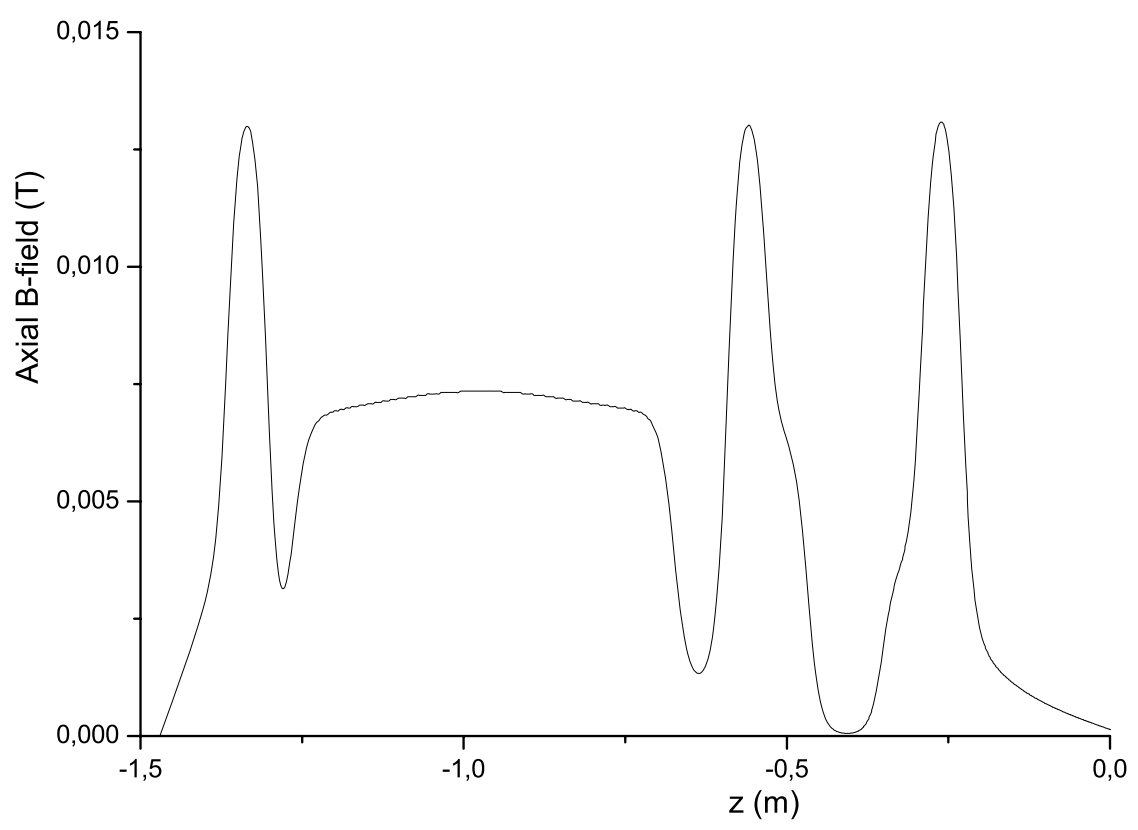

Fig. 3: Axial magnetic field before bending magnet

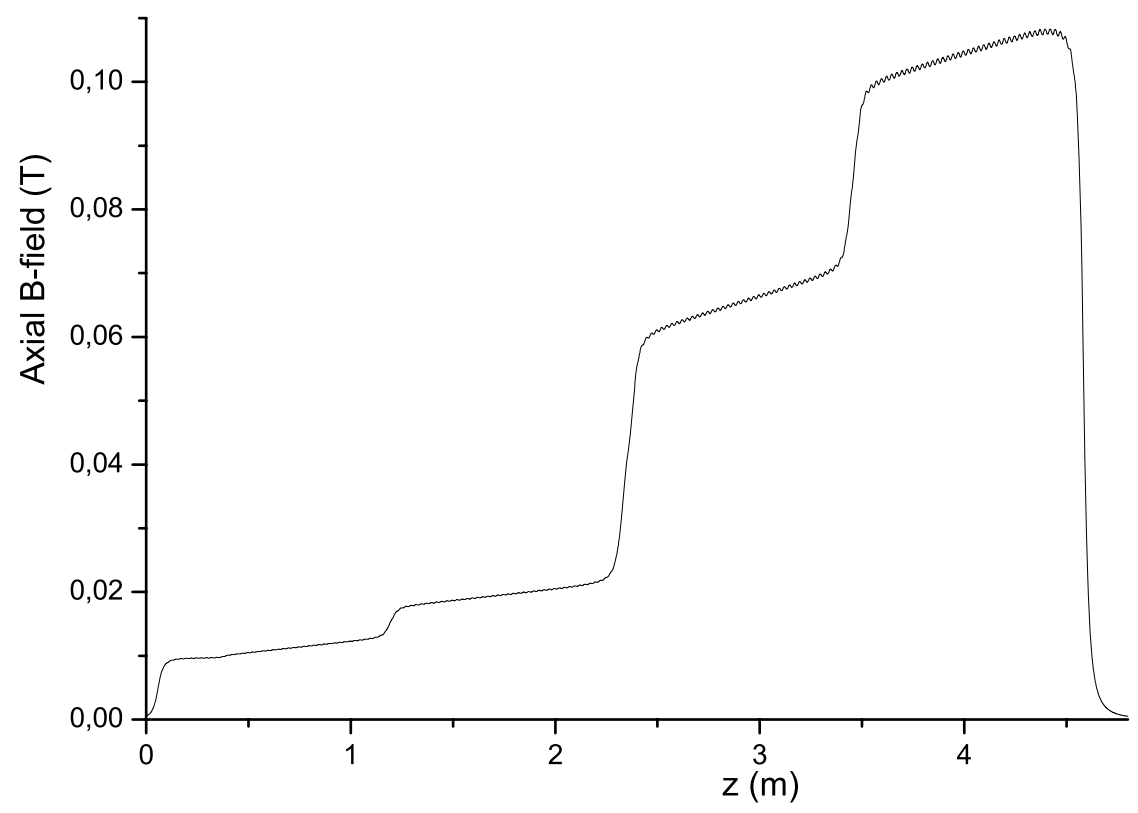

Fig. 4: Axial magnetic field after bending magnet 


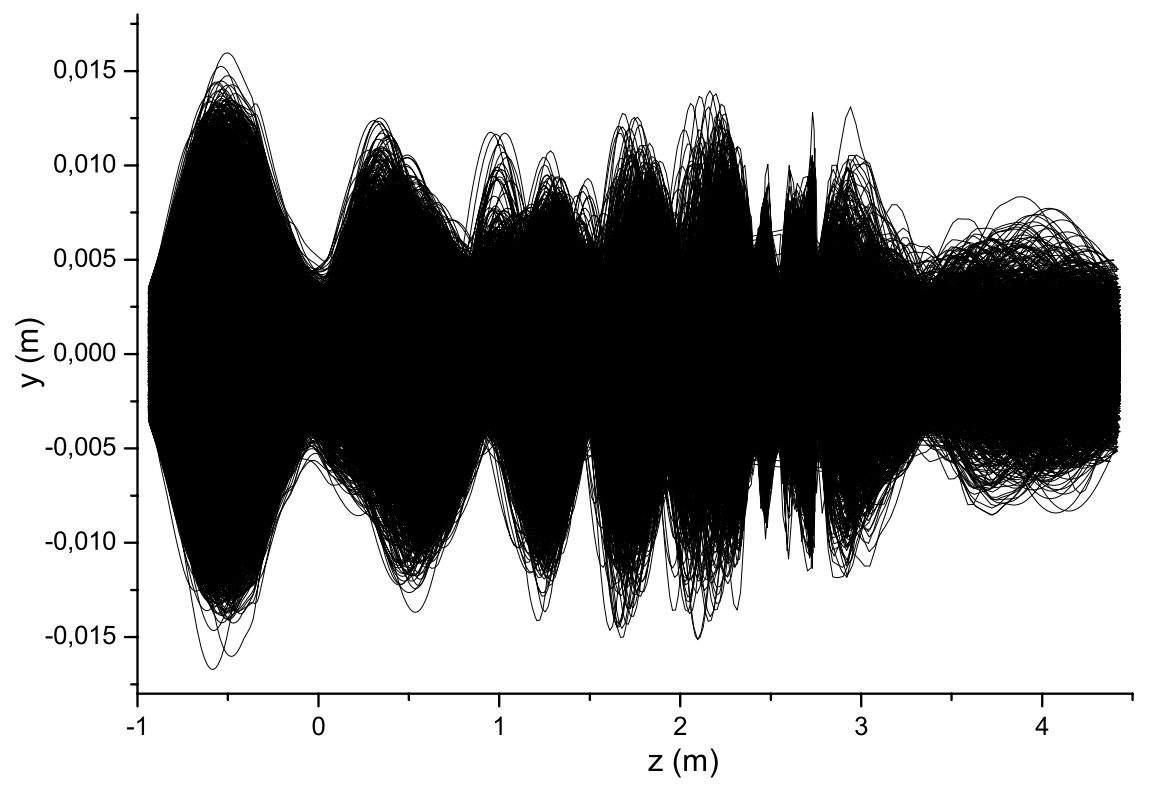

Fig. 5: Macro particle trajectories (in y-z plane) from gun exit through bunching section

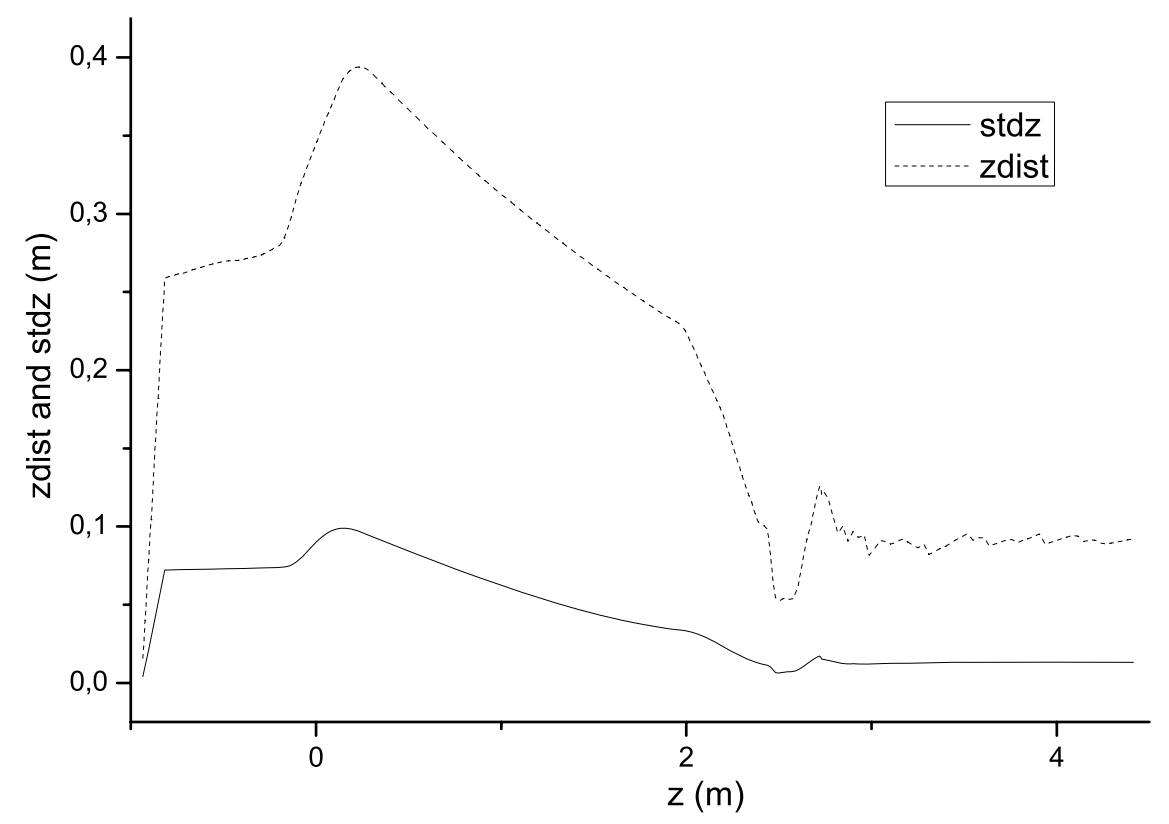

Fig. 6: Maximum axial particle distance (zdist) and standard deviation of z-coordinates (stdz) 


\section{Tables}

\begin{tabular}{|l|c|c|c|c|c|}
\hline Parameter & SHB1 & SHB2 & L-Band cell & $\begin{array}{c}4 \text { cell L-band } \\
\text { Buncher }\end{array}$ & $\begin{array}{c}5 \text { cell L-band } \\
\text { Buncher }\end{array}$ \\
\hline Frequency [MHz] & 108 & 433 & 1300 & 1300 & 1300 \\
\hline Phase angle $\left.{ }^{\circ}{ }^{\circ}\right]$ & 100 & 120 & 216 & 190 & 170 \\
\hline Voltage $[\mathrm{kV}]$ & 65 & 70 & & & 11.4 \\
\hline Field strength $\mathrm{E}_{\mathrm{z}}[\mathrm{MV} / \mathrm{m}]$ & & & 12.3 & 11.4 & \\
\hline
\end{tabular}

Table 1: Optimum operating parameters for the bunching system 


\title{
Analysis of Off-Nuclear X-Ray Sources in Galaxy NGC 4945
}

\author{
Sarah M. Harrison \\ Office of Science, Science Undergraduate Laboratory Internship (SULI) \\ Massachusetts Institute of Technology \\ Stanford Linear Accelerator Center \\ Stanford, CA
}

August 24, 2006

Prepared in partial fulfillment of the requirements of the Office of Science, Department of Energy's Science Undergraduate Laboratory Internship under the direction of Grzegorz Madejski and Martin Mueller at the Kavli Institute for Particle Astrophysics and Cosmology, Stanford Linear Accelerator Center.

Participant:

Signature

Research Advisor:

Signature 


\section{TABLE OF CONTENTS}

Abstract

$\begin{array}{ll}\text { Introduction } & 1\end{array}$

$\begin{array}{ll}\text { Materials and Methods } & 3\end{array}$

$\begin{array}{lr}\text { Results } & 6\end{array}$

$\begin{array}{ll}\text { Discussion and Conclusions } & 7\end{array}$

$\begin{array}{lr}\text { Acknowledgments } & 10\end{array}$

$\begin{array}{ll}\text { References } & 10\end{array}$ 


\begin{abstract}
Analysis of Off-Nuclear X-Ray Sources in Galaxy NGC 4945. SARAH M. HARRISON (Massachusetts Institute of Technology, Cambridge, MA 02139) GRZEGORZ MADEJSKI AND MARTIN MUELLER (Kavli Institute for Particle Astrophysics and Cosmology, Stanford Linear Accelerator Center, Stanford, CA 94025)
\end{abstract}

Recently, X-ray astronomy has been used to investigate objects such as galaxies, clusters of galaxies, Active Galactic Nuclei (AGN), quasars, starburst superbubbles of hot gas, X-ray binary systems, stars, supernova remnants, and interstellar and intergalactic material. By studying the x-ray emission patterns of these objects, we can gain a greater understanding of their structure and evolution. We analyze X-ray emission from the galaxy NGC 4945 using data taken by the Chandra X-ray Observatory. The Chandra Interactive Analysis of Observations (CIAO) software package was used to extract and fit energy spectra and to extract light curves for the brightest off-nuclear sources in two different observations of NGC 4945 (January, 2000 and May, 2004). A majority of sources were closely fit by both absorbed power law and absorbed bremsstrahlung models, with a significantly poorer $\chi^{2} /$ dof for the absorbed blackbody model, and most sources had little variability. This indicates that the sources are accreting binary systems with either a neutron star or black hole as the compact object. The calculated luminosities were about $10^{38} \mathrm{erg} / \mathrm{s}$, which implies that the mass of the accreting object is close to 10 solar masses and must be a black hole. 


\section{INTRODUCTION}

The study of X-ray sources provides information about the structure of the objects which make up the universe. Recently, X-ray data has been used to investigate objects such as galaxies, clusters of galaxies, Active Galactic Nuclei (AGN), quasars, starburst superbubbles of hot gas, X-ray binary systems, stars, supernova remnants, and interstellar and intergalactic material. By studying the X-ray emission patterns of these objects, we can gain a greater understanding of their structure and evolution. The process of accretion (the extraction of gravitational potential energy from a material which falls onto a gravitating body) is known to be the principal source of power in several types of binary systems and is also believed to power AGN and quasars [1]. The study of luminous x-ray sources can provide further insight into the accretion process in stellar systems.

There are three main mechanisms of astronomical X-ray production: thermal bremsstrahlung in an ionized gas, synchrotron radiation, and blackbody radiation. The acceleration of charged particles during collisions within a hot, ionized gas causes emission of radiation known as thermal bremsstrahlung. This radiation forms an energy continuum with a characteristic shape dependent on the temperature of the gas. It also may have characteristic emission lines based on the elemental composition of the gas. This type of radiation is often emitted from hot gas in clusters of galaxies and supernova remnants. Synchrotron radiation, also known as magnetic bremsstrahlung, is generated when a relativistic electron is accelerated by a magnetic field. An example of a synchrotron emitter is the central region of the Crab Nebula, a young supernova remnant in our galaxy, which has a very strong magnetic

field ( $10^{-4}$ gauss) [2]. X-rays are also produced by blackbody radiation of normal stars over a broad temperature range $(2,500 \mathrm{~K}$ to $40,000 \mathrm{~K})[2]$ and high-temperature neutron stars, among other objects. By measuring the x-ray spectrum and the shape of the continuum by fitting it with one of these models, the source emission can be classified as one of these types.

Measuring the luminosity of the sources can also give a lower limit for the mass of the 
objects, and possibly lead to the discovery of extremely luminous or massive off-nuclear sources. For many accreting astrophysical systems, there is a maximum luminosity which the system reaches. As material accretes onto a compact object, the gravitational potential energy lost is released in the form of electromagnetic radiation. This radiation exerts a force on the surrounding material, mostly on free electrons through Thompson scattering. At a point known as the Eddington limit, the outward force from the radiation balances the inward gravitational force, preventing any further increase in the rate of accretion. By balancing the radiative and gravitational forces, the mass of systems radiating at their Eddington luminosity can be determined[1].

Seyfert galaxies are spiral or irregular galaxies containing a strong nucleur source, most likely a supermassive black hole. Seyferts are a type of active galaxy, galaxies which emit a large fraction of their energy through a bright, compact, extremely massive galactic center. NGC 4945 is a nearby (3.7 Mpc)[3] Seyfert 2 galaxy known to have a very luminous AGN. The behavior of this nuclear region is currently the subject of detailed study. The spectra of the nucleus, starburst, and superwind regions were isolated in order to study the current model of black hole and accretion disk in Seyfert 2 galaxies[4]. In addition, a powerful offnuclear source has been observed in NGC 4945 which is thought to be an ultra-powerful x-ray binary or a supernova remnant[3].

In this paper, a number of off-nuclear sources in NGC 4945 will be investigated and catalogued using x-ray data taken by the Chandra X-ray Observatory (CXO). The classification of these sources will increase the depth of knowledge about X-ray binaries and other emitters and may lead to the discovery of new and interesting X-ray sources such as massive off-nuclear black holes. The discovery and analysis of ultraluminous sources, sources with luminosities greater than $2 * 10^{39} \mathrm{erg} / \mathrm{s}[5]$, can provide more information about the physics of intermediate mass black holes, black holes with masses between 10 and 100 solar masses. Only a few of these type of sources are known, and little is understood about their evolution. Other possible sources of these X-rays are supernova remnants, normal stars, and 
binary systems involving accreting white dwarf stars, neutron stars, or stellar black holes.

\section{MATERIALS AND METHODS}

\section{The Chandra Satellite}

The Chandra X-ray Observatory is composed of a high-resolution X-ray telescope and a number of advanced imaging and spectroscopic instruments. Launched on July 9, 1999, the Chandra satellite achieved the most advanced spatial and X-ray spectral resolution to date. The satellite is comprised of the Pointing Control and Aspect Determination (PCAD) system, which controls the pointing of the observatory, the High Resolution Mirror Assembly (HRMA), which focuses the X-rays, and the Science Instrument Module (SIM), which houses the two focal instruments, the High Resolution Camera (HRC) and the Advanced CCD Imaging Spectrometer (ACIS). In addition are the Low Energy Transmission Grating (LETG) and High Energy Transmission Grating (HETG) for use with the HRC and ACIS, respectively.

ACIS, the camera used for the observations analyzed in this paper, is composed of 10 planar 1024-by-1024 pixel charge coupled devices (CCDs) arranged in two arrays. One is a four-chip array called ACIS-I and the other is a six-chip array called ACIS-S. ACIS can obtain high-resolution images and moderate resolution spectra, but the use of ACIS-S in conjunction with the HETG can obtain very high resolution spectra. The HETG is composed of 2 sets

of gratings: the medium energy grating (MEG) and the high energy grating (HEG). Overall, the HETG is designed for high resolution spectroscopy between $0.4 \mathrm{keV}$ and $10.0 \mathrm{keV}[6]$.

\section{Data Acquisition and Analysis}

X-ray sources in the nearby galaxy NGC 4945 were analyzed and compared using data taken by the Chandra satellite during two observations, one in 2000 and one in 2004 . The first observation began on January 27, 2000 (total exposure time $49.75 \mathrm{ks}$ ) with the ACIS camera 
in faint mode and did not use a grating. The second observation began on May 28, 2004 and was split into two data sets (total exposure times $78.64 \mathrm{ks}$ and $95.8 \mathrm{ks}$, with $57.6 \mathrm{ks}$ gap). Both observations were taken with the ACIS camera in faint mode and with a high energy transmission grating (HETG) placed in front of the camera. The data was retrieved from the Chandra archive and analyzed using the Chandra Interactive Analysis of Observations (CIAO) software package[7]. Two main files for each observation were used in data analysis. The first is a source file, a table of all the sources detected, their locations in the sky, their net count rate and background counts, along with other information. The second is an event file, which allows the extraction of an image of net photon events at each pixel position, like the one shown in figure 1 . The earlier observation had to be reprocessed because many of the software threads had been updated since the original processing in 2000. This included the removal of afterglow effects caused by residual charge left by the interaction of cosmic rays with the CCD. This excess charge is captured by charge traps and persists through several subsequent frames of CCD read-out and could result in spurious detection of faint sources. Certain pixels which may have been illuminated by extra charge were recorded in an updated file in order to remove them from data analysis. The event and source files provided for the 2004 observations had already been processed with the most recent techniques in CIAO and did not need to be reprocessed.

The brightest X-ray sources in each of the observations were determined using source files provided by the Chandra archive. The images of NGC 4945 were displayed with the event file. This image was used to locate and extract circular regions (of radius 5 pixels) around each of the brightest sources in order to extract energy spectra for these sources. In addition, a source-free background region for each observation was used to subtract a background count from the source regions when producing spectra. A tool from the CIAO package was used to extract these spectra as well as the Auxilary Response File (ARF) and Redistribution Matrix File (RMF) for each source. These files are used during spectral fitting to take into account the effective detector area and detector ineffeciencies in energy 
resolution, as well as the transition from incoming photon energy to the value recorded by the detector. These spectra display the photon energy versus the number of photons in each energy range and can be used to determine the nature of the X-ray emission. The spectral fitting technique used assumes a model with reasonable parameters, propogates it through the instrument by taking into account the $\mathrm{ARF}$ and $\mathrm{RMF}$, and compares this output to the data. Then the parameters are adjusted until a minimum $\chi^{2}$ value is reached.

Three different fit models were applied to each source spectrum using the program xspec. Absorbed power law, absorbed bremsstrahlung, and absorbed blackbody models were compared to each other for each of the sources, and model parameter values and $\chi^{2} /$ dof were calculated for each. For each model, the flux was used to calculate the luminosity of the source by the formula:

$$
L=4 \pi r^{2} f
$$

where $f=$ the flux of the source and $r$ is the distance to the galaxy (3.7 Mpc).

This calculated luminosity was used to determine a lower limit for the mass of each source and to indicate whether a black hole or a neutron star was present for those sources which were accreting x-ray binary systems.

Light curves, plots of the change in net photon count rate with time over the course of the observation, were extracted and plotted for these sources using CIAO. In each case, the plots were binned in $3000 \mathrm{~s}$ intervals. In order to take into account the grating used in observations 4899 and 4900, the curves were rescaled using the count rates calculated for the nuclear source in each of the observations which is known not to be variable. The light curves were used to study the emission rate of each source and to assess its variability. Both the light curves and energy spectra for each observation were compared for each source in order to study its time evolution. In addition to the brightest sources, sources which were present in one observation and not detectable in another were analyzed. A catalog of the brightest sources in the galaxy NGC 4945 was produced. 


\section{RESULTS}

Table 1 displays spectral fitting information for the five sources with the greatest count rate (listed in order) in each observation. These sources are labeled in order of decreasing count rate in observation 864 and keep this same number across observations. The position of each source is provided in units of right ascention and declination, and the count rate is measured within the energy range $0.5-10 \mathrm{keV}$ (the range used for fitting). The spectral parameters for each of the three fits used are displayed, including $n_{H}$, the absorption column density for the absorbed component of each model, the photon index for the power law model, and the temperature for the bremsstrahlung and blackbody models. The $\chi^{2} /$ dof value calculated for each model is displayed, along with the flux calculated for each fit (in the energy range 2-10 $\mathrm{keV}$ ) and the luminosity of the source. In observation 864, the four brightest sources were best fit by absorbed thermal bremsstrahlung models, and the fifth brightest source was best fit by an absorbed power law model. In each case, the $\chi^{2} /$ dof values for absorbed power law or bremsstrahlung were fairly close to each other and significantly better than that for the absorbed blackbody model. The luminosities for these sources ranged from $9 * 10^{38} \mathrm{erg} / \mathrm{s}$ for the brightest source to $2 * 10^{38} \mathrm{erg} / \mathrm{s}$ for the fifth brightest source.

Except for the fifth brightest source (source 6) in observation 4899, where each model had a $\chi^{2} /$ dof of 1.5 , the $\chi^{2} /$ dof values for absorbed power law and bremsstrahlung models remained similar and significantly lower than that for the absorbed blackbody model. Sources 3, 7, and 5 in observation 4900 also show this characteristic.

The spectral parameters for sources 1 through 7 in the observations in which they were not one of the five brightest sources are displayed in table 2 . Source 7 was not bright enough to be detected in observation 864. Two moderately bright sources were observed in observation 864 but were not noticeable in observations 4899 and 4900. The spectral parameters for these sources are displayed in table 3.

Figures 2 through 10 display the lightcurves for sources 1 through 9 in each of the 
observations in which they were noticed. Most sources, except for source 7 which shows high variability, show little or no variability over the course of each observation.

\section{DISCUSSION AND CONCLUSIONS}

Most sources were best fit with absorbed power law and absorbed bremsstrahlung models, which have similar shape. The fit of absorbed power law indicates that these sources are accreting x-ray binaries, with either a neutron star or black hole as the compact object. The luminosity range of $2-9 * 10^{38} \mathrm{erg} / \mathrm{s}$ indicates masses of a few solar masses, stars which are most likely black holes. The hydrogen column density calculated for absorption for most

models is $2-7 * 10^{21} \mathrm{~cm}^{-2}$, which is smaller than the $n_{H}$ calculated by Done to be $4 * 10^{24}$ $\mathrm{cm}^{-2}$ and closer to the reported absorption of our own galaxy, which is $2 * 10^{21} \mathrm{~cm}^{-2}[4]$.

Because observations 4899 and 4900 were taken with the high energy transmission grating in front of the ACIS camera, fewer photon events were recorded for each source than in observation 864, and the overall count rates were smaller. Statistically, the fits to the data from observation 864 were more accurate because they incorporated more events. The sparsity of counts in the later observations may have caused the fitting to be less accurate, especially with the fainter sources, where the number of pha bins used for fitting was less than 25, whereas the faintest source in 864 had 50 pha bins. In addition the light curves for observation 864 reflect the variability of the sources more accurately because more counts were included in each bin.

Source 1: This source is one of the five most luminous sources in each observation. It was consistently fit reasonably well by both the absorbed power law and absorbed bremsstrahlung models. The count rate decreased by an order of magnitude between observation 864 (0.059 cts/s) and observations $4899(0.0064 \mathrm{cts} / \mathrm{s})$ and $4900(0.0060 \mathrm{cts} / \mathrm{s})$. The luminosity also decreased significantly between the observations, going from $9 * 10^{38} \mathrm{erg} / \mathrm{s}$ in 864 , a luminosity on the order of ultraluminous sources $\left(10^{39} \mathrm{erg} / \mathrm{s}\right)$ to 5 or $6 * 10^{38} \mathrm{erg} / \mathrm{s}$ in 4899 and 4900 . 
This suggests that the luminosity of this source is decreasing.

Source 2: In all three observations, this source was best fit by an absorbed bremsstrahlung model. The absorbed power law fit was also a close fit, with a photon index consistently between 2.3 and 2.5. The luminosity of this source also significantly decreased between observation $864\left(5.6 * 10^{38} \mathrm{erg} / \mathrm{s}\right)$ and observations 4899 and $4900\left(1.7 * 10^{38} \mathrm{erg} / \mathrm{s}\right)$, suggesting this source is getting fainter.

Source 3: This source, observed to be the third brightest in observation 864, was the most luminous in observations 4899 and 4900 . It was best fit by an absorbed bremsstrahlung model in all three observations, but was also closely fit by an absorbed power law model. For the power law model, photon index was consistently between 1.8 and 2.0. The luminosity increased from about $4 * 10^{38} \mathrm{erg} / \mathrm{s}$ in observation 864 to over $5 * 10^{38} \mathrm{erg} / \mathrm{s}$ in observations 4899 and 4900, indicating an increase in brightness of this source.

Source 4: The luminosity of this source remained consistent between all three observations, with a value between 2.5 and $2.9 * 10^{38} \mathrm{erg} / \mathrm{s}$ for the best fit models, which were absorbed bremsstrahlung and absorbed power law. This source does not show any variability.

Source 5: Like all of the other sources mentioned so far, this source was best fit by both absorbed power law and absorbed bremsstrahlung models. Its luminosity is consistent between the three observations, with a value between 1.7 and $2.1 * 10^{38} \mathrm{erg} / \mathrm{s}$. This source does not show any variability.

Source 6: This source, which had the fifth highest count rate in observation 4899, could not be well fit with any of the models for this observation. The $\chi^{2} /$ dof for each model was $>$ 1.5. In observations 864 and 4900, this source was best fit by an absorbed power law model with photon index of 1.5 and 2.5, respectively, and luminosities of $2.4 * 10^{38} \mathrm{erg} / \mathrm{s}$ and $3.3 * 10^{38} \mathrm{erg} / \mathrm{s}$, respectively. Because of the low count rate this source had in observation 4899 and 4900, it was more difficult to fit accurately.

Source 7: This source was too faint in observation 864 to extract an analysis, was observable in observation 4899, and was the third brightest source in observation 4900. In both 
observations 4899 and 4900, it was best fit by an absorbed power law model. Its luminosity was observed to increase from $2.0 * 10^{38} \mathrm{erg} / \mathrm{s}(4899)$ to $4.4 * 10^{38} \mathrm{erg} / \mathrm{s}(4900)$ over the short span between the two observations. The fact that it was barely observable in observation 864 indicates that it may have been flaring during the time of observations 4899 and 4900 .

Source 8: The absorbed bremsstrahlung model provided the best fit for this source, and gave a calculated luminosity of $1.3 * 10^{38} \mathrm{erg} / \mathrm{s}$. The absorbed power law model also provided a relatively good fit, with photon index of 2.4 and a similar calculated luminosity of $1.5 * 10^{38} \mathrm{erg} / \mathrm{s}$. This source, one of the fainter ones in observation 864, was not observable in observations 4899 and 4900, indicating a variable luminosity.

Source 9: This source is the only one which was best fit with the absorbed blackbody model. It was also reasonably well fit by the absorbed bremsstrahlung model. The faintest source described here, this source had a luminosity between 1.1 and $1.3 * 10^{37} \mathrm{erg} / \mathrm{s}$ during observation 864 and was not noticeable in observations 4899 and 4900, indicating a variable luminosity.

With luminosities in the range $<10^{39} \mathrm{erg} / \mathrm{s}$, it is unlikely that these sources are ultraluminous X-ray sources. The luminosities of these sources are in the same range $\left(10^{38} \mathrm{erg} / \mathrm{s}\right)$ as the luminosities of about 5 of the black hole candidates in our own galaxy as reported in Tanaka and Lewin[8]. The ratio of the luminosities of the sources in NGC 4945 to the Eddington luminosity for a source of one solar mass is a little under 10, and because it is unlikely that the sources are radiating at their Eddington limit (therefore this ratio underestimates the mass), these sources must be accreting black holes of almost 10 solar masses. The evidence of slight variability in the light curves rules out the possibility that most of these sources are supernovae, which would have very stable light curves without any variability. It can be concluded that NGC 4945 has more off-axis black holes than our own galaxy, which are in accreting binary systems and are emitting X-rays at luminosities close to the threshhold for ultraluminous X-ray sources.

A comparison with the analysis by Done et al.[4] on the nuclear source in NGC 4945 
verifies the analysis techniques used in this paper. Table 5 displays a comparison of the spectral parameters found by modeling the nuclear source with an absorbed powerlaw + gauss model in all three observations with the parameters for the model obtained by [4]. In each observation the presence of the FeK $\alpha$ line closely matches the $6.4 \mathrm{keV}$ value found by Done et al. The photon index is not similar between the two separate analyses, but this may have to do with the difference in the definition of the extraction region between the two different methods.

Further study of the variability of the sources could further confirm the conclusion that the brightest sources reported here are indeed black hole X-ray binaries. In addition, it could indicate more about the astrophysical process powering these X-ray sources which is most likely accretion. A search for optical sources at the same coordinates in observations taken by the Hubble telescope could provide more accurate information about the luminosities of these sources, as well as their activity in the optical band.

\section{ACKNOWLEDGMENTS}

I would like to thank my mentor, Dr. Grzegorz Madejski, and Martin Mueller for giving me guidance in this project. I would also like to thank Adam Edwards and all who read and aided me in writing this paper. Finally, I would like to thank the Department of Energy for sponsoring the SULI program and supporting youth in scientific research.

\section{REFERENCES}

[1] J. Frank et al., Accretion Power in Astrophysics, 2nd ed. Cambridge University Press, 1992.

[2] P. A. Charles and F. D. Seward, Exploring the X-Ray Universe. Cambridge University Press, 1995. 
[3] W. N. Brandt et al., "A powerful and highly variable off-nuclear x-ray source in the composite starburst/seyfert 2 galaxy ngc 4945," Mon. Not. R. Astron. Soc., vol. 281, pp. L41-L45, 1996.

[4] C. Done et al., "Simultaneous chandra and rossi x-ray timing explorer observations of the nearby bright seyfert 2 galaxy ngc 4945," The Astrophysical Journal, vol. 558, pp. $763-770,2003$.

[5] H. Feng and P. Kaaret, "Xmm-newton observations of ultraluminous x-ray sources in nearby galaxies," The Astrophysical Journal, vol. 633, pp. 1052-1063, 2005.

[6] The Chandra Proposers' Observatory Guide, 2nd ed. Chandra X-Ray Center, Chandra Project Science MSFC, and Chandra IPI Teams, 2000.

[7] "Chandra x-ray observatory," 2006. [Online]. Available: http://chandra.harvard.edu

[8] Y. Tanaka and W. H. G. Lewin, "Black-hole binaries," X-Ray Binaries, 1995. 


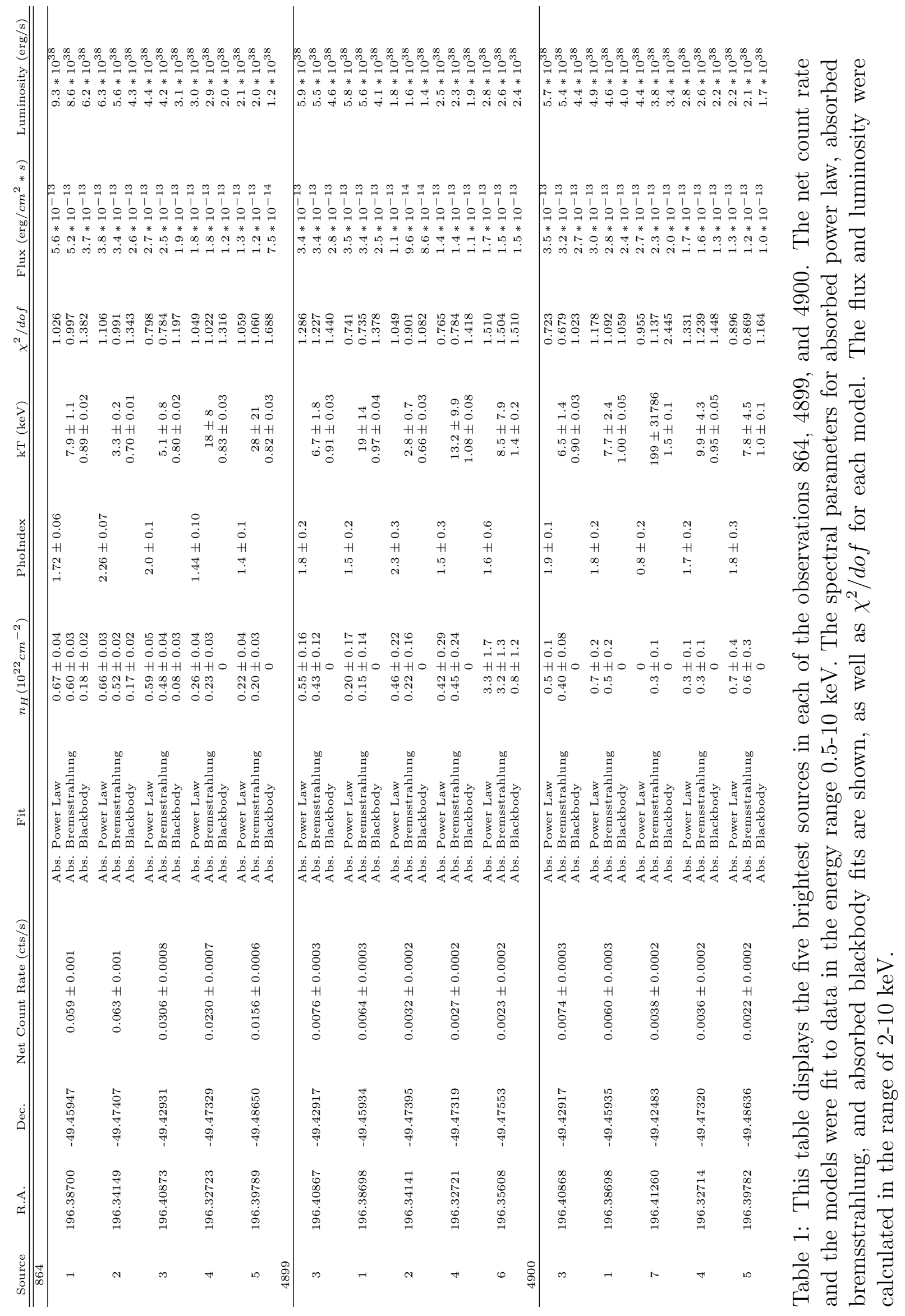




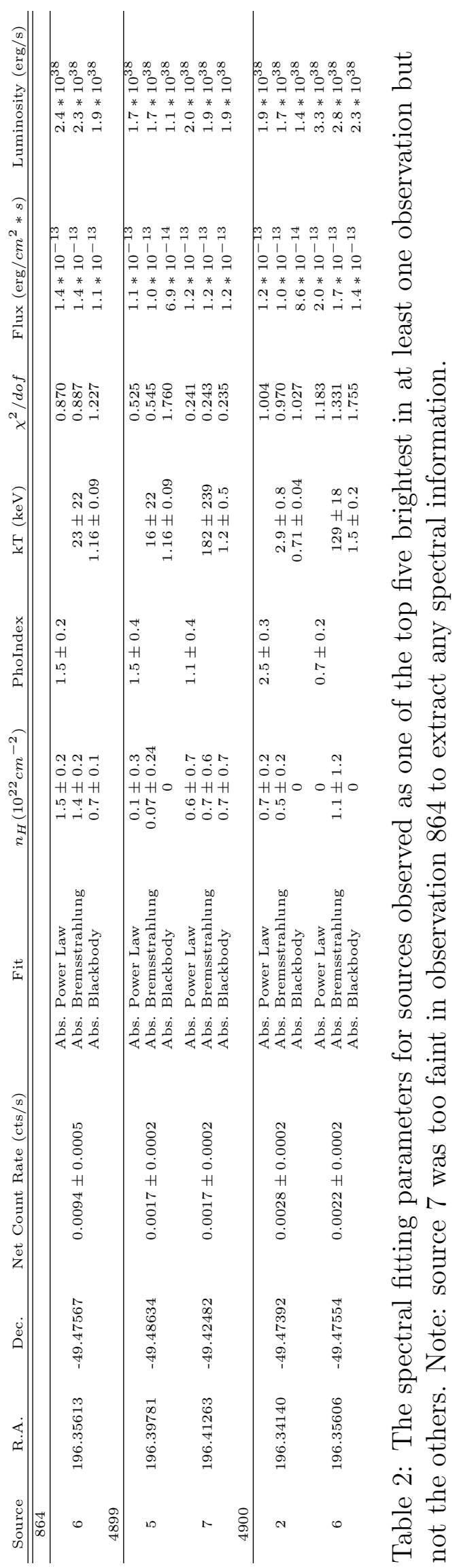




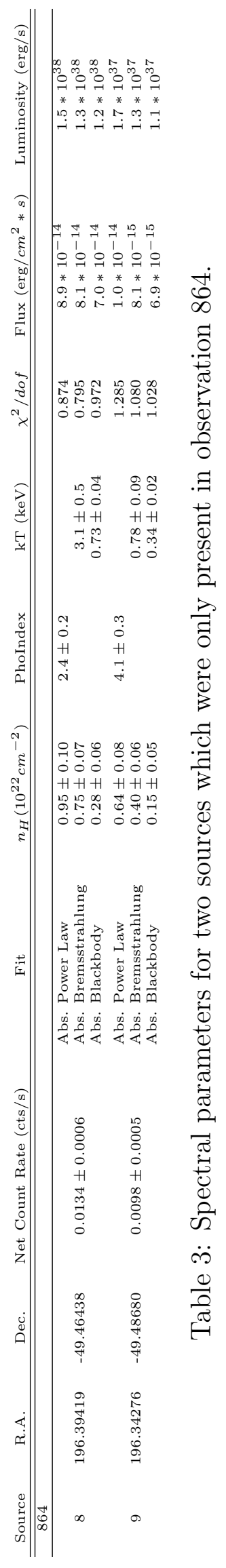




\section{FIGURES}

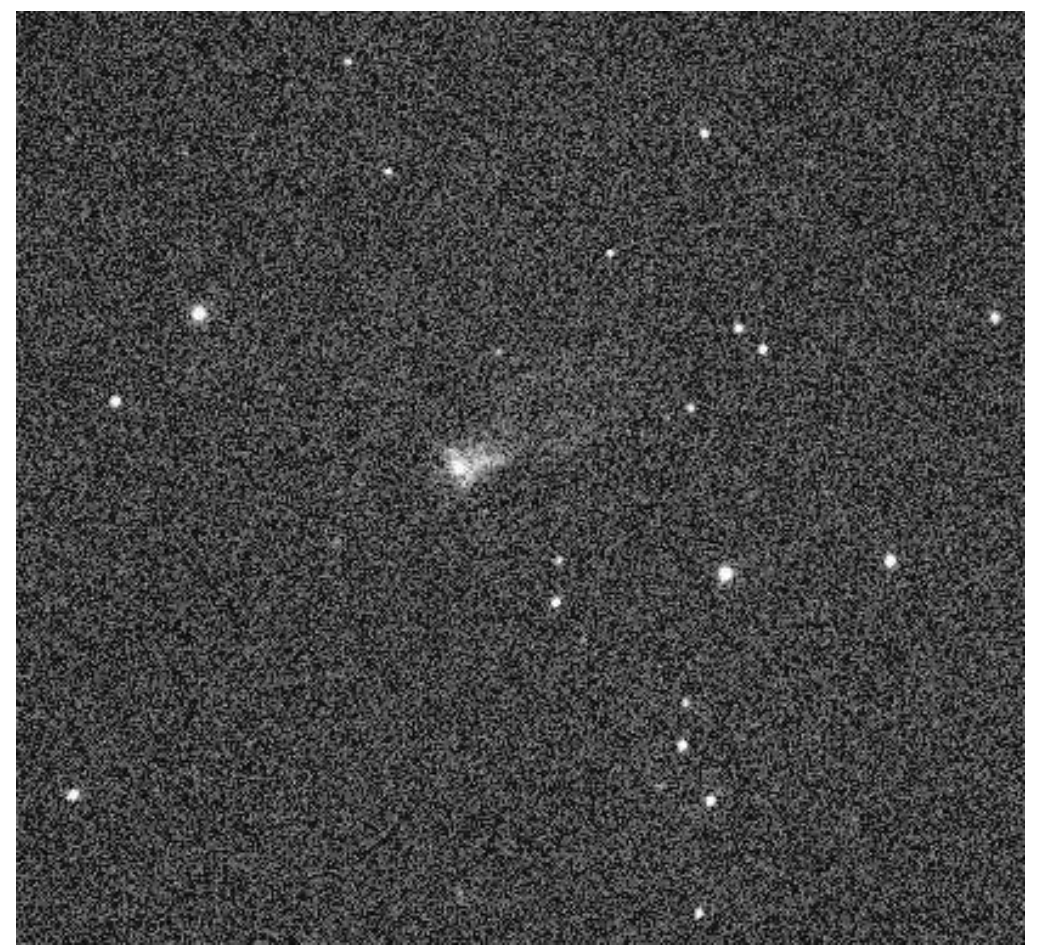

Figure 1: Chandra ACIS image of NGC 4945 from observation 864. 

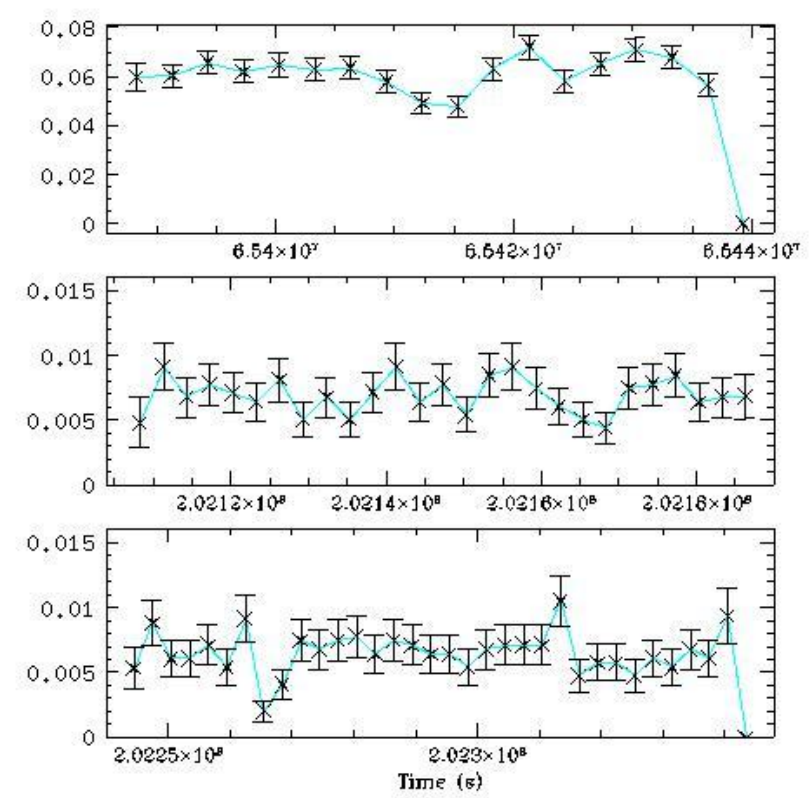

Figure 2: Source 1 light curves for observations 864, 4899, and 4900.

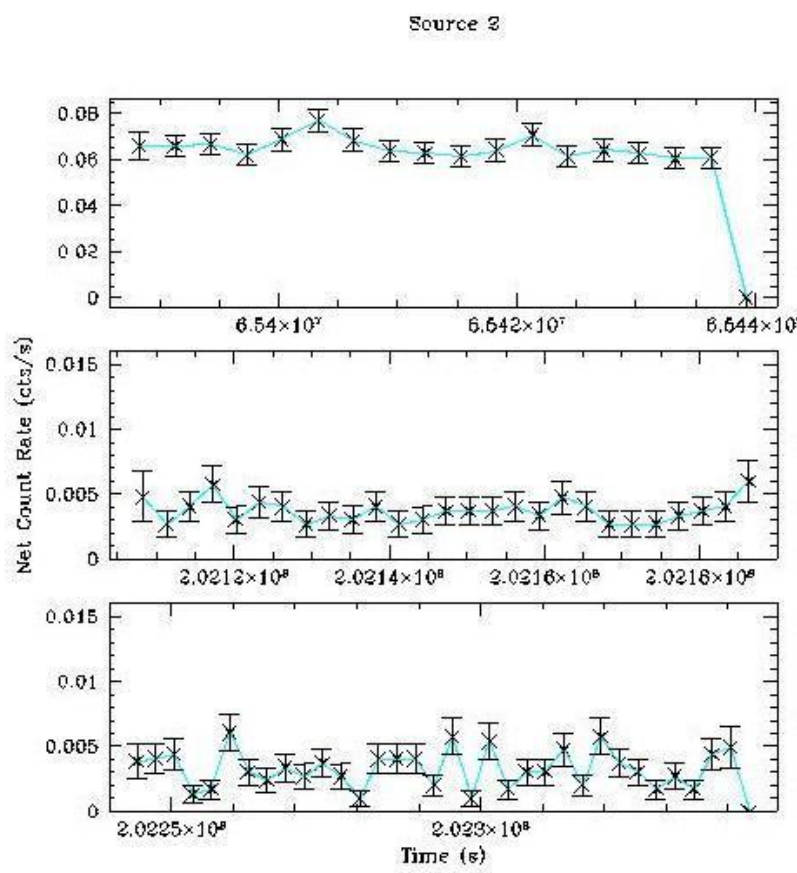

Figure 3: Source 2 light curves for observations 864, 4899, and 4900. 
Source 3
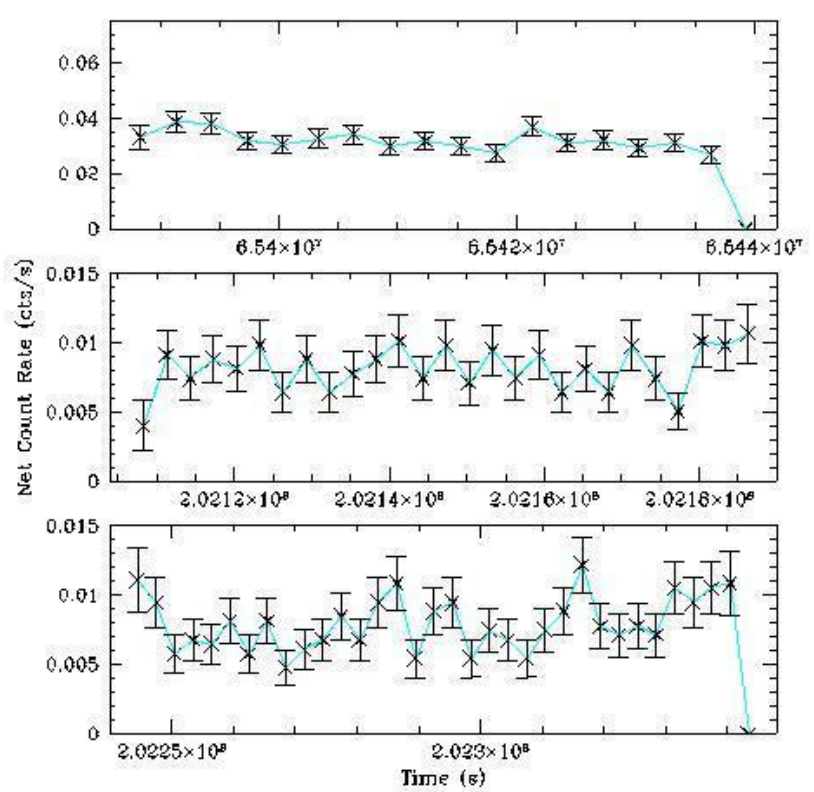

Figure 4: Source 3 light curves for observations 864, 4899, and 4900.

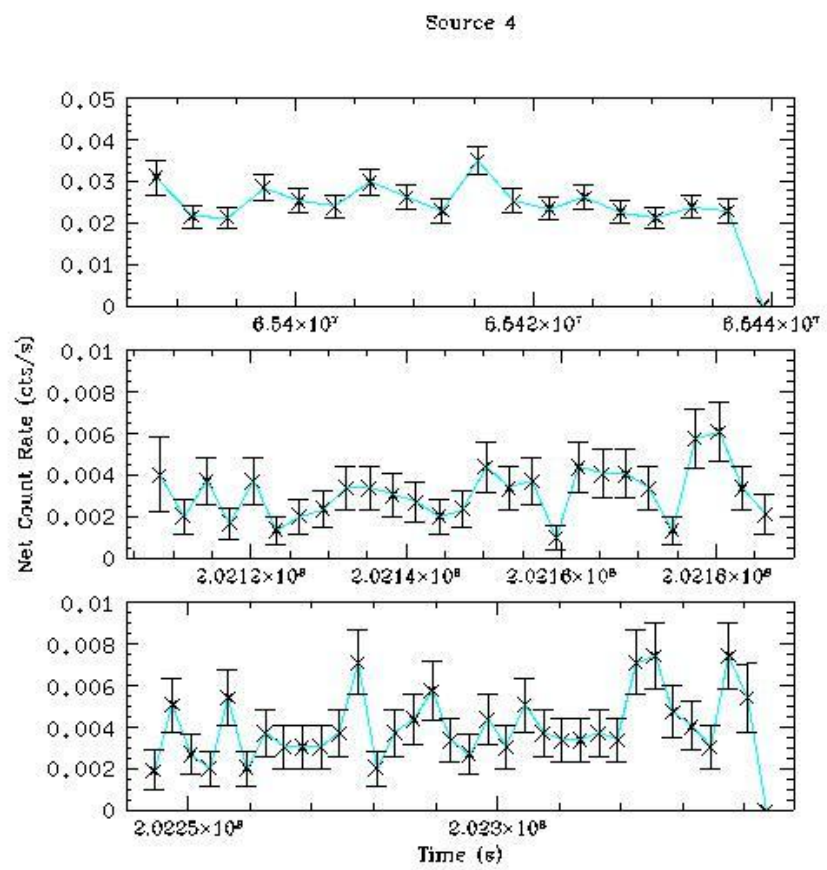

Figure 5: Source 4 light curves for observations 864, 4899, and 4900. 


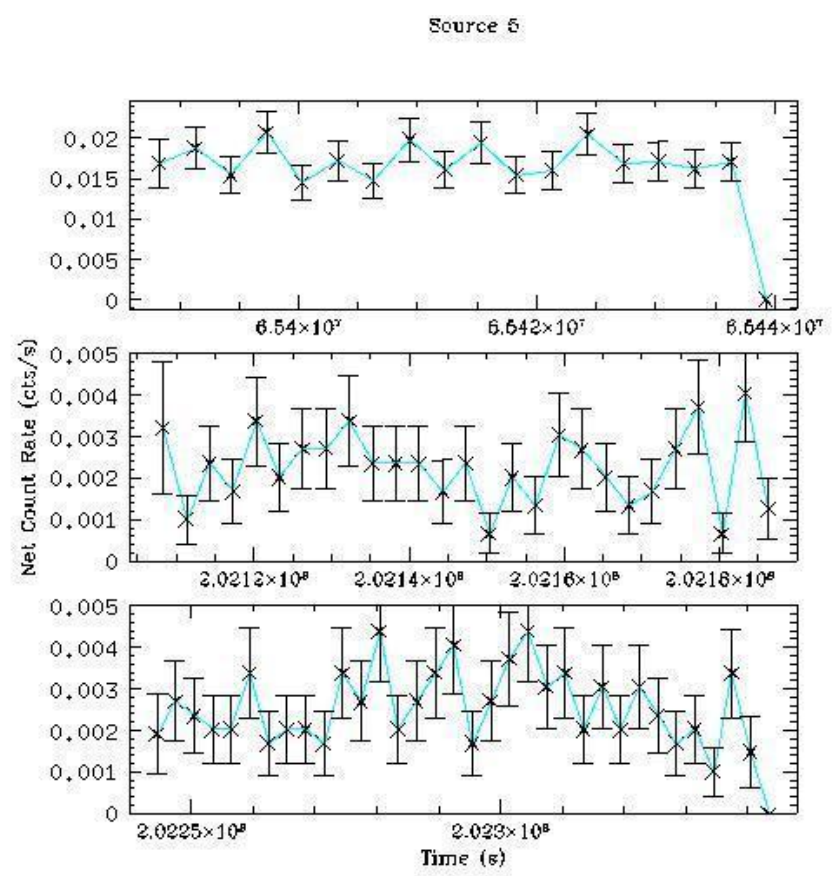

Figure 6: Source 5 light curves for observations 864, 4899, and 4900.

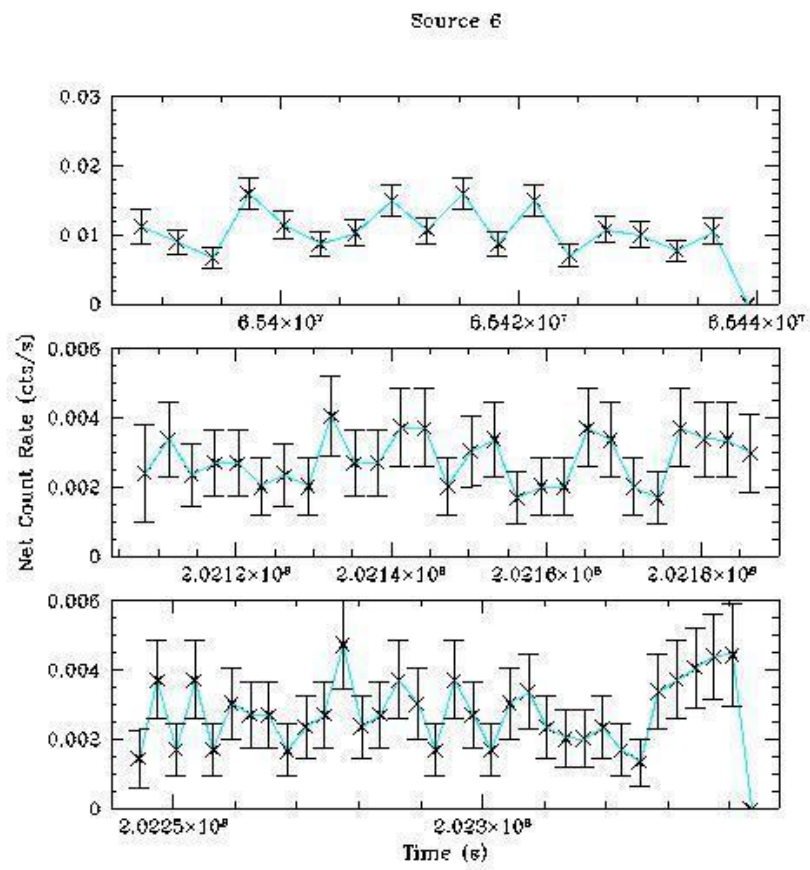

Figure 7: Source 6 light curves for observations 864, 4899, and 4900. 

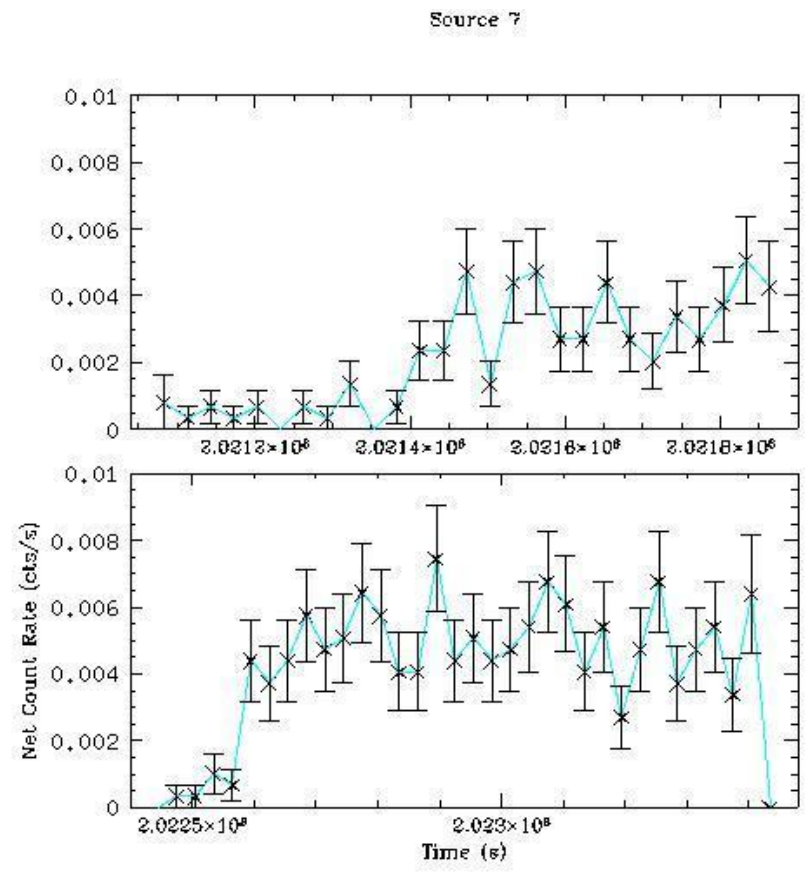

Figure 8: Source 7 light curves for observations 4899 and 4900.

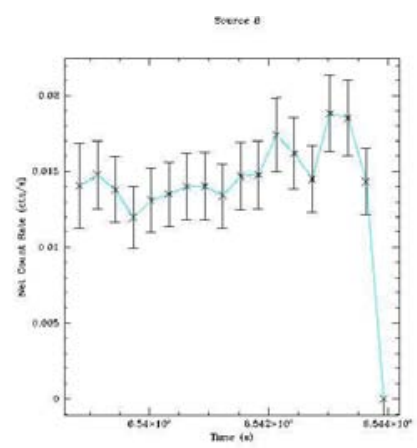

Figure 9: Source 8 light curve for observation 864. 


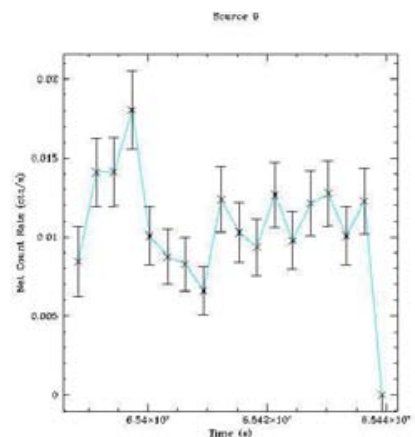

Figure 10: Source 9 light curve for observation 864 . 


\section{TABLES}
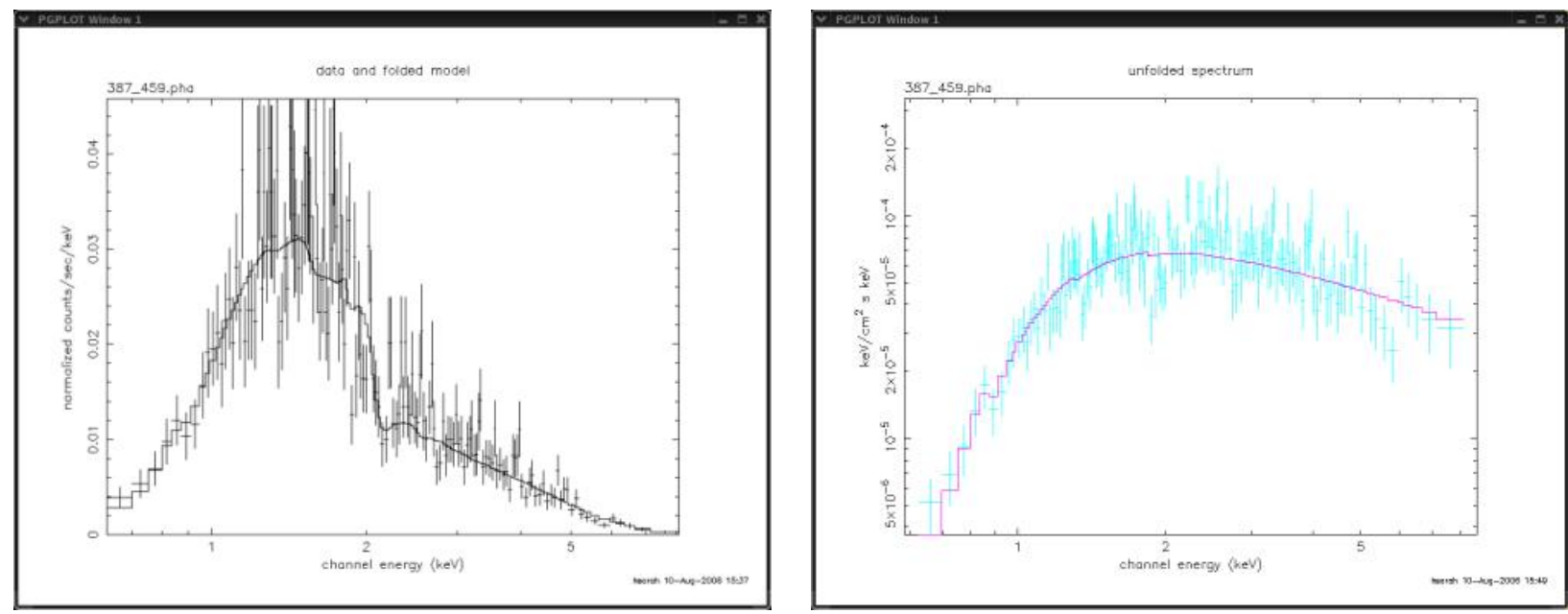

Table 4: Folded spectrum (left) and unfolded spectrum (right) for source 1 in observation 864, fitted with power law model.

\begin{tabular}{c|c|c|c||c} 
& 864 & 4899 & 4900 & Done et al. \\
\hline \hline$n_{H}\left(10^{22} \mathrm{~cm}^{-2}\right)$ & $1.1 \pm 0.2$ & $1.4 \pm 0.5$ & $1.4 \pm 0.5$ & $0.8 \pm 0.8$ \\
PhoIndex & $0.03 \pm 0.1$ & $-0.1 \pm 0.2$ & $-0.03 \pm 0.2$ & $1.65 \pm 0.15$ \\
FeK $\alpha(\mathrm{keV})$ & $6.4 \pm 0.2$ & $6.41 \pm 0.01$ & $6.385 \pm 0.007$ & 6.4 \\
$\chi^{2} /$ dof & 1.61 & 1.23 & 1.69 & 0.95
\end{tabular}

Table 5: Comparison of spectral parameters for fitting of nuclear source with absorbed powerlaw + gauss model between observations 864, 4899, and 4900 with those obtained by Done et al.. 


\title{
Limited Streamer Tube System for Detecting Contamination in the Gas Used in the BABAR Instrumented Flux Return
}

\author{
Laura Ingalls Huntley \\ SLAC-TN-06-024 \\ Office of Science, SULI Program \\ Franklin \& Marshall College \\ Stanford Linear Accelerator Center \\ Menlo Park, California
}

August 25, 2006

Prepared in partial fulfillment of the requirements of the Office of Science, U.S. Department of Energy Science Undergraduate Laboratory Internship (SULI) Program under the direction of Dr. Mark Convery in the BABAR group at the Stanford Linear Accelerator Center.

Participant:

Signature

Research Advisor:

$$
\text { Signature }
$$




\section{Table of Contents}

$\begin{array}{ll}\text { Abstract } & 3\end{array}$

$\begin{array}{ll}\text { Introduction } & 4\end{array}$

Materials and Methods $\quad 5$

$\begin{array}{ll}\text { Results } & 7\end{array}$

$\begin{array}{lr}\text { Discussion and Conclusions } & 8\end{array}$

$\begin{array}{ll}\text { Acknowledgements } & 9\end{array}$

$\begin{array}{lr}\text { References } & 9\end{array}$

$\begin{array}{ll}\text { Tables } & 10\end{array}$

$\begin{array}{ll}\text { Figures } & 11\end{array}$ 


\begin{abstract}
Limited Streamer Tube System for Detecting Contamination in the Gas Used by the BABAR Instrumented Flux Return. High Energy Physics, Office of Science, SULI Program, Stanford Linear Accelerator Center, Summer 2006. LAURA HUNTLEY (Franklin \& Marshall College, Lancaster, PA 17603) MARK CONVERY (Stanford Linear Accelerator Center, Menlo Park, CA 94025).
\end{abstract}

The Resistive Plate Chambers (RPCs) initially installed in the Instrumented Flux Return (IFR) of the BABAR particle detector have proven unreliable and inefficient for detecting muons and neutral hadrons. In the summer of 2004, the BABAR Collaboration began replacing the RPCs with Limited Streamer Tubes (LSTs). LST operation requires a mixture of very pure gases and an operating voltage of $5500 \mathrm{~V}$ to achieve maximum efficiency. In the past, the gas supplies obtained by the BABAR Collaboration have contained contaminants that caused the efficiency of the IFR LSTs to drop from approximately 90\% to approximately 60\%. Therefore, it was necessary to develop a method for testing this gas for contaminants. An LST test system was designed and built using two existing LSTs, one placed $1 \mathrm{~cm}$ above the other. These LSTs detect cosmic muons in place of particles created during the BABAR experiment. The effect of gas contamination was mimicked by reducing the operating voltage of the test system in order to lower the detection efficiency. When contaminated gas was simulated, the coincidence rate and the percent coincidence between the LSTs in the test system dropped off significantly, demonstrating that test system can be used as an indicator of gas purity. In the fall of 2006, the LST test system will be installed in the gas storage area near the BABAR facility for the purpose of testing the gas being sent to the IFR. 


\section{INTRODUCTION}

The BABAR Collaboration at the Stanford Linear Accelerator Center endeavors to measure fundamental differences between matter and anti-matter through the study of electronpositron collisions at high energy. These collisions are designed to create pairs of B and anti-B mesons, which in turn decay into other hadrons and leptons. By measuring the lifetimes of the B mesons and identifying their decay products, BABAR physicists are able to study charge parity violation, as well as many other interesting physical phenomena [1].

The outermost component of the BABAR particle detector is an Instrumented Flux Return (IFR), which shapes the magnetic field as well as provides identification for muons and neutral hadrons produced by the electron-position collisions. The IFR consists of layers of particle detectors fitted between layers of steel [2]. Muons, which only interact electromagnetically with matter, are able to travel through more of the steel layers than other particles, making it possible to identify them [3]. Originally, the detectors in the IFR were a new, cost-effective technology called Resistive Plate Chambers (RPCs). RPCs consist of parallel plastic plates at high voltage (HV). When a particle passes through the gas mixture between these plates, ionization occurs, and a streamer signal is produced [4]. Since irregularities in the surfaces of the plates can also cause electron flow to occur, the plates are coated with linseed oil in order to keep them smooth. Unfortunately, the efficiency of the BABAR RPCs has decreased rapidly, most likely due to defects in the plate surfaces and contaminants in the linseed oil, and they have become unreliable for detecting particles. As attempts made to repair them were unsuccessful, two of the six RPC sectors were replaced with Limited Streamer Tubes (LSTs) in the summer of 2004. The remaining four sectors will be installed in the fall of 2006 (See Figure 1). 
The LSTs contain long cells filled with a gas composed of 3\% argon, $8 \%$ isobutane, and 89\% carbon dioxide by volume [5]. When charged particles enter the LSTs, they ionize the gas, causing a small number of free electrons to be produced. These electrons drift toward the HV wires running through the tubes. If the voltage is large enough, a cascade of electrons is created, causing an electronic response in the $\mathrm{HV}$ wires. However, if the voltage is excessive, secondary cascades will be produced, causing false signals. With pure gas and operating at the optimal voltage of 5500V, the LSTs are approximately 90\% efficient at detecting particles. The gas used in the LSTs is bought by SLAC from an outside source and mixed on-site at a gas storage area near the BABAR facility. In the past, the isobutane supplies have contained impurities that caused the detection efficiency of the IFR to drop to approximately 60\%. It was determined that, in order to prevent efficiency loss in the future, a method for testing the purity of the gas was needed [6].

In the summer of 2006, we designed and assembled an LST test system for the purpose of detecting contaminated gas before it enters the BABAR IFR. This system detects cosmic muons, which pass through horizontal surfaces at an approximate rate of $1 \mathrm{~cm}^{-2} \mathrm{~min}^{-1}$ [7], in place of particles produced by electron-positron collisions. The accompanying electronics make it possible to measure the coincidence rate, or the number of detections made by both LSTs per unit time, and the percent coincidence, or the ratio of coincidental detections to the number of detection made by the top LST. As the number of coincidental detections is proportional to the product of the efficiencies of the LSTs, both the rate and percent of coincidence are very sensitive to decreased efficiency in the LSTs and are thus good indicators of contaminated gas. 


\section{MATERIALS AND METHODS}

Two LST modules, one resting $1 \mathrm{~cm}$ above the other and each measuring $50.5 \mathrm{~cm}$ x 15.4 $\mathrm{cm}$ x $2.0 \mathrm{~cm}$, were used to create the gas testing system (See Figure 2). The LSTs are configured such that gas may be pumped through the LSTs in series and out through a bubbler. The gas

used during our test was bought pre-mixed from an off-site company. Because it was impractical to pollute this gas for testing purposes, impure gas was simulated by decreasing the voltage in the top LST below the optimum range.

Aside from being significantly shorter, the LSTs in the testing system are identical to those used in the BABAR IFR. Each LST consists of eight long cells, which are coated with a graphite paint kept at ground potential. Each cell contains a gold-plated wire at variable HV. These wires are paired into four input-output channels on the end of the LST (See Figure 3). A HV connector box plugs into each of these channels as well as a ground connection. The frontend electronics are housed in an Ortec Nuclear Instruments \& Methods (NIM) Bin, Model 401B (See Figure 4). HV from a Bertan HV Power Supply, Model 1739P, is fed into the connector box and travels through a $1 \mathrm{M} \Omega$ resistor to the LST. If a particle triggers an electronic cascade, the analog signal travels back into the connector box and through a $1 \mathrm{nF}$ capacitor to the frontend electronics (See Figure 5).

The front-end electronics take signals from the LSTs and use NIM modules to measure the number of muons passing through the LSTs. A LeCroy Octal Discriminator, Model 623B, receives the signals from the connector box and outputs a fixed-width pulse for every signal above the threshold voltage, which we set at approximately $30 \mathrm{mV}$. A LeCroy 4-Fold Logic Unit, Model 365AL, uses OR gates to bundle the channels of each LST together. The signals 
from the OR gates are then sent to a LeCroy Dual Gate Generator, Model 222, which output square-wave pulses of width $10 \mu$ s. The signal from the top LST then goes to a Joerger Visual Scalar, Model VS, where the number of pulses is counted. It is also sent, along with the signal from the bottom LST, to a LeCroy Quad Coincidence Gate, Model 622, where an AND gate sends a pulse to another counter in the Visual Scalar if the two LSTs detect a muon at the same time. Figure 6 shows a block diagram of the front-end electronics.

The coincidence rate can be measured by counting the number of coincidental detections in a known period of time. The percent coincidence can be found by dividing the coincidental counts by the number of detections made by the top LST. In order to increase the stability and magnitude of the coincidence count, only the inner two channels in the top LST were used, as they are more likely to detect muons that will also pass through the bottom LST.

\section{RESULTS}

We measured the average number of cosmic muons passing through the top LST to be $(1.261 \pm .059) \mathrm{cm}^{-2} \mathrm{~min}^{-1}$ and the bottom LST to be $(1.229 \pm .022) \mathrm{cm}^{-2} \mathrm{~min}^{-1}$. These flux measurements agree with literature value of approximately $1 \mathrm{~cm}^{-2} \mathrm{~min}^{-1}$ [7] and demonstrate that our LSTs are functioning properly.

The number of muons a single LST detects per some length of time as a function of the voltage supplied to the LST is called a "singles rate test." For this test, we varied the voltage from $4800 \mathrm{~V}$ to $5500 \mathrm{~V}$ and recorded the number of detections in 10 seconds. Functional LSTs usually show an edge on this curve at $5300 \mathrm{~V}$ and a plateau around $5500 \mathrm{~V}$ [5]. We found that our LSTs meet these expectations (see Figure 7). 
With pure gas and with both LSTs operating at the optimal voltage of $5500 \mathrm{~V}$, we measured a $(241.6 \pm 6.5) \mathrm{min}^{-1}$ coincidence rate and $(46.6 \pm 1.1) \%$ coincidence. Table 1 shows the changes in coincidence rate and percent coincidence over a period of several hours.

Figures 8 and 9 show the coincidence rate and the percent coincidence as a function of the voltage in the top LST while the bottom LST is held at $5500 \mathrm{~V}$. We conducted these tests by varying the voltage in the top LST from $4800 \mathrm{~V}$ to $5500 \mathrm{~V}$ and measuring the number of coincidental detections and the number of detections made by the top LST during a period of five minutes. As can be seen in the Figures, both the coincidence rate and the percent coincidence increase as the voltage, and therefore the efficiency, of the top LST approaches the optimal voltage range and then decrease as the excessive voltage begins to create false signals.

\section{DISCUSSION AND CONCLUSIONS}

We conclude that our LSTs presently meet performance expectations on the evidence of their measurements of muon flux and the singles rate test. In the future, however, it will be necessary to repeat these tests on a case-by-case basis in order to determine whether any efficiency losses are actually the result of impure gas and not due to LST malfunction.

As can been seen from Figures 8 and 9, both the coincidence rate and the percent coincidence are sensitive to changes in LST efficiency. The coincidence rate test has the advantage of not depending on the detections made by a single LST module, which is important since x-rays can sometimes be detected by one LST and cause the percent coincidence to drop despite the fact that no efficiency losses have occurred. On the other hand, the percent coincidence is a simpler test to perform, as the coincidence rate test requires the use of a 
stopwatch or timer. Furthermore, while the coincidence rate is theoretically more reliable, Table 1 shows that the percent coincidence did not vary significantly over a period of several days.

We recommend that both tests be used according to the needs and time-constraints of the test system operator. For both tests, measurements should be made for at least five minutes in order to obtain sufficiently accurate and precise results. The test system will be installed as an intermediary between the gas storage area and the main BABAR detector in the fall of 2006, where it will be used to check gas being sent to the BABAR IFR for contamination

\section{ACKNOWLEDGEMENTS}

This research was conducted at the Stanford Linear Accelerator Center. I thank the U. S. Department of Energy, Office of Science for providing with the opportunity to participate in the exciting research conducted at SLAC. I also thank my mentor, Mark Convery, for his guidance and advice. Finally, I thank the coordinators and participants of the Science Undergraduate Laboratory Internships program at SLAC for their effort, dedication, and friendship.

\section{REFERENCES}

[1] P.F. Harrison and H.R. Quinn, Eds., The BABAR Physics Book, Menlo Park, California: Stanford Linear Accelerator Center, 1998.

[2] B. Aubert, et al., "The BABAR Detector," Nuclear Instruments and Methods in Physics Research A, vol. 479, pp.1-116, 2002.

[3] J. Perl, et al., "The BABAR Detector," [Online document], May 2006, [cited 2006 Aug. 11], Available at HTTP: http://www.slac.stanford.edu/BFROOT/www/doc/workbook /detector/detector.html. 
[4] R. Santonico and R. Cardarelli, "Development of Resistive Plate Counter,” Nuclear Instruments and Methods, vol. 187, pp. 377-380, 1981.

[5] W. Menges, “The BABAR Muon System Upgrade,” Nuclear Science Symposium Conference Record, 2005 IEEE, vol. 3, pp. 1470-74.

[6] M. Convery, "LST Operations and Installation Plans," presented at the BABAR Collaboration Meeting, Montreal, QC, 2006.

[7] W.-M. Yao, et al., "2006 Review of Particle Physics,” Journal of Physics G, vol. 33, no. 1, Jul., pp. 1-1232.

\section{TABLES}

\begin{tabular}{|l|l|l|}
\hline Time & Coincidence Rate $\left(\mathrm{min}^{-1}\right)$ & Percent Coincidence (\%) \\
\hline \hline 0 days 0 hrs. & $242.8 \pm 7.0$ & $44.96 \pm .96$ \\
0 days 1.5 hrs. & $250.4 \pm 7.1$ & $47.39 \pm .97$ \\
5 days 3 hrs. & $235.0 \pm 6.9$ & $46.37 \pm .99$ \\
5 days 4 hrs. & $250.2 \pm 7.1$ & $48.34 \pm .98$ \\
6 days 4 hrs. & $233.2 \pm 6.8$ & $45.82 \pm .99$ \\
6 days 5 hrs. & $238.4 \pm 6.9$ & $46.06 \pm .98$ \\
7 days 4.5 hrs. & $238.6 \pm 6.9$ & $46.17 \pm .98$ \\
7 days 6 hrs. & $244.2 \pm 7.0$ & $47.64 \pm .99$ \\
\hline
\end{tabular}

Table 1. Coincidence rate and percent coincidence taken at different times over a period of several days. The mean of the coincidence rate is $(241.6 \pm 6.5) \mathrm{min}^{-1}$ and the mean of the percent coincidence is $(46.6 \pm 1.1) \%$. 


\section{FIGURES}

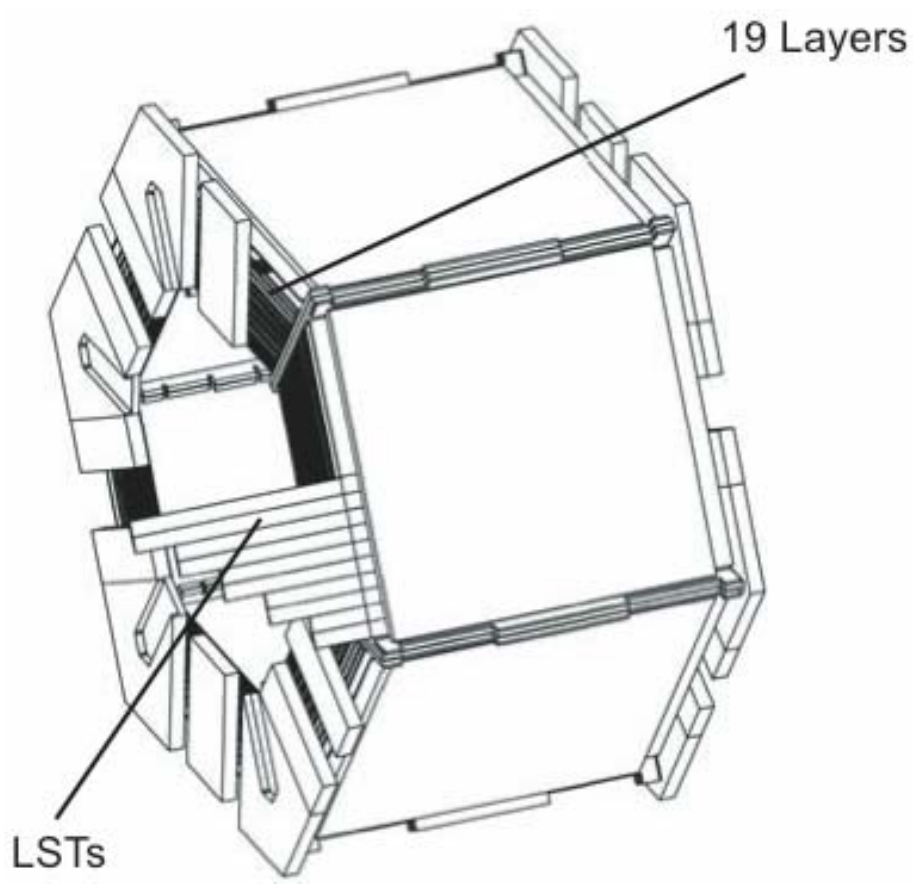

Figure 1. LSTs being inserted into the gaps between the steel layers of the BABAR IFR.

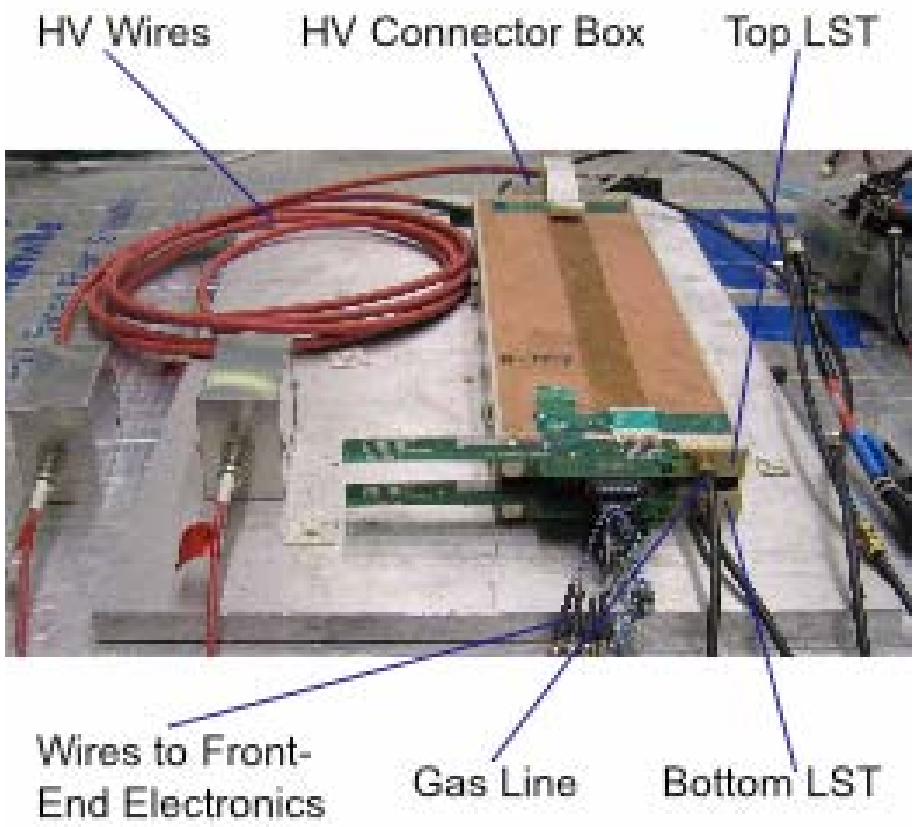

Figure 2. LST test system consisting of two LSTs, one placed $1 \mathrm{~cm}$ above the other. 


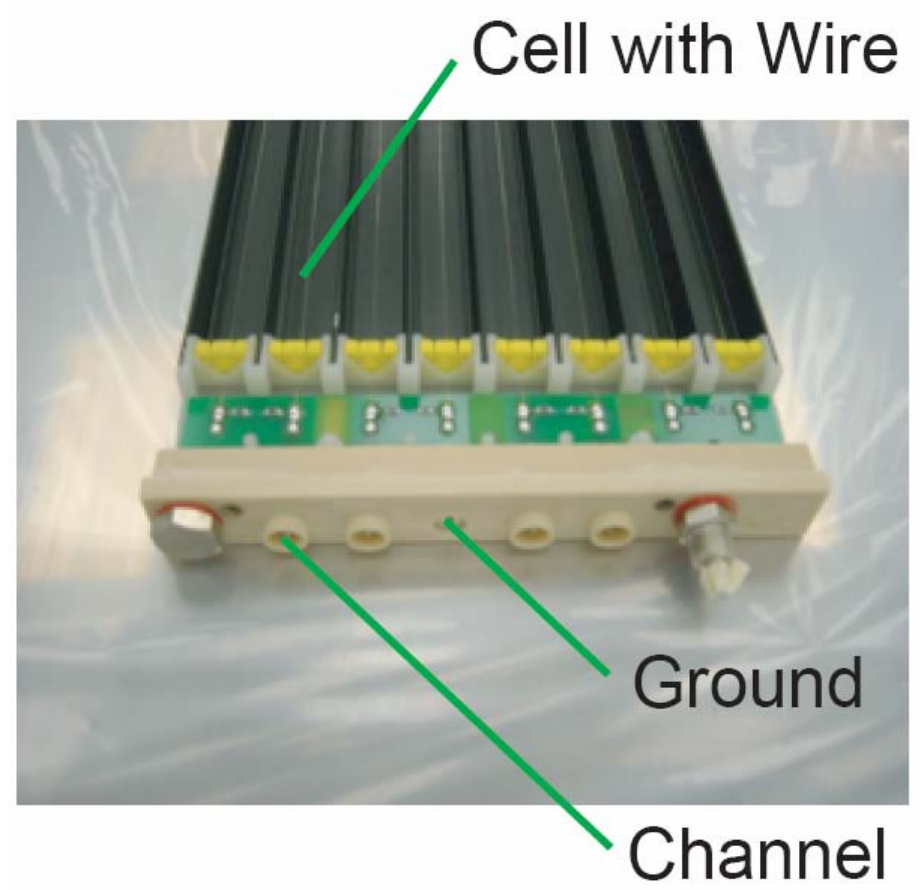

Figure 3. LST shown without plastic sleeve. Eight cells coated in a graphite-based paint contain one gold-plated wire each. Two wires comprise one channel. The end-cap of the LST has plugs for four channels, along with a ground pin.

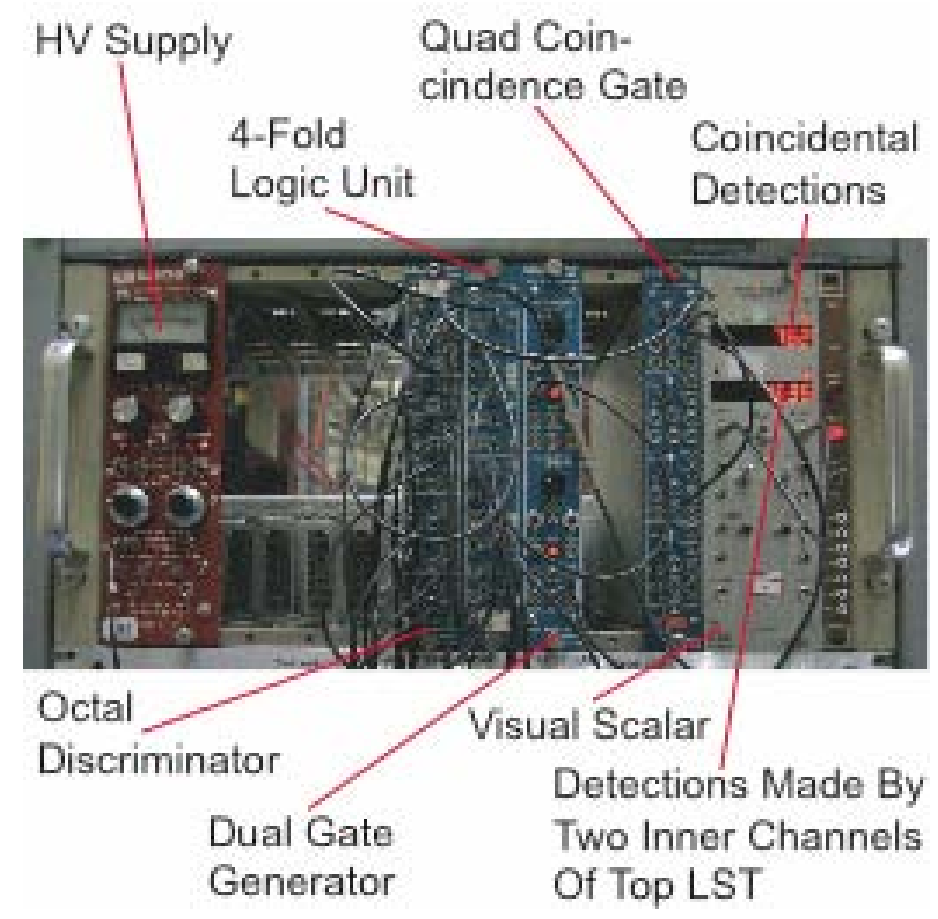

Figure 4. Ortec NIM Bin, Model 401B, with HV power supply and front-end electronics. 

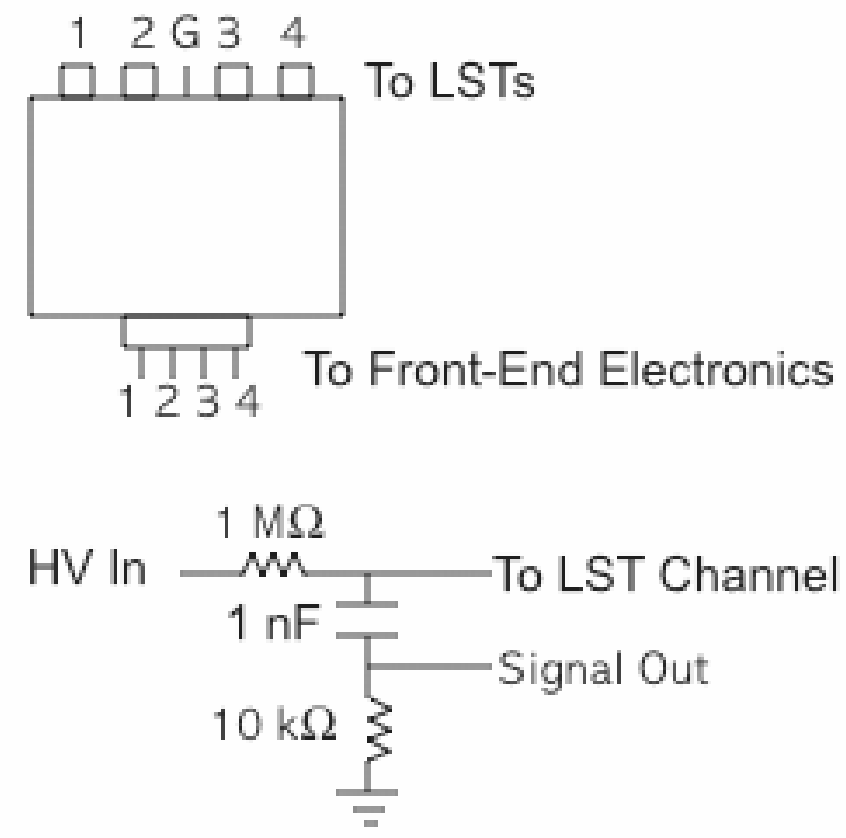

Figure 5. HV Connector Box. The top diagram shows connector box with four channels and a ground plug, plus four output wires to the front-end electronics. The bottom diagram shows the circuitry for one channel in the connector box.

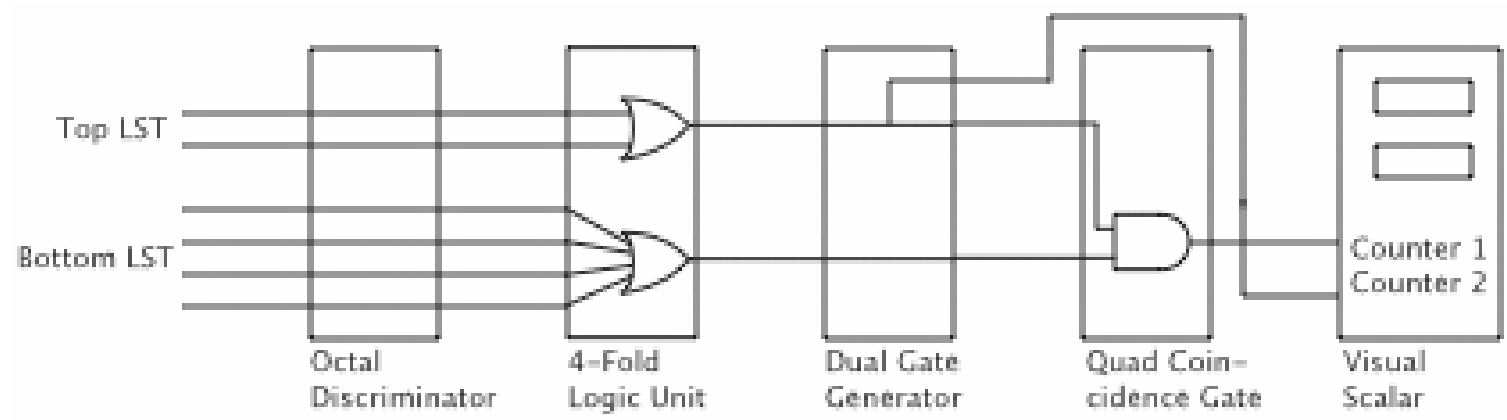

Figure 6. Block diagram of front-end electronics. Counter 1 displays the number of detections made coincidentally by both LSTs and Counter 2 displays the number of detections made by the inner two channels of the top LST. 


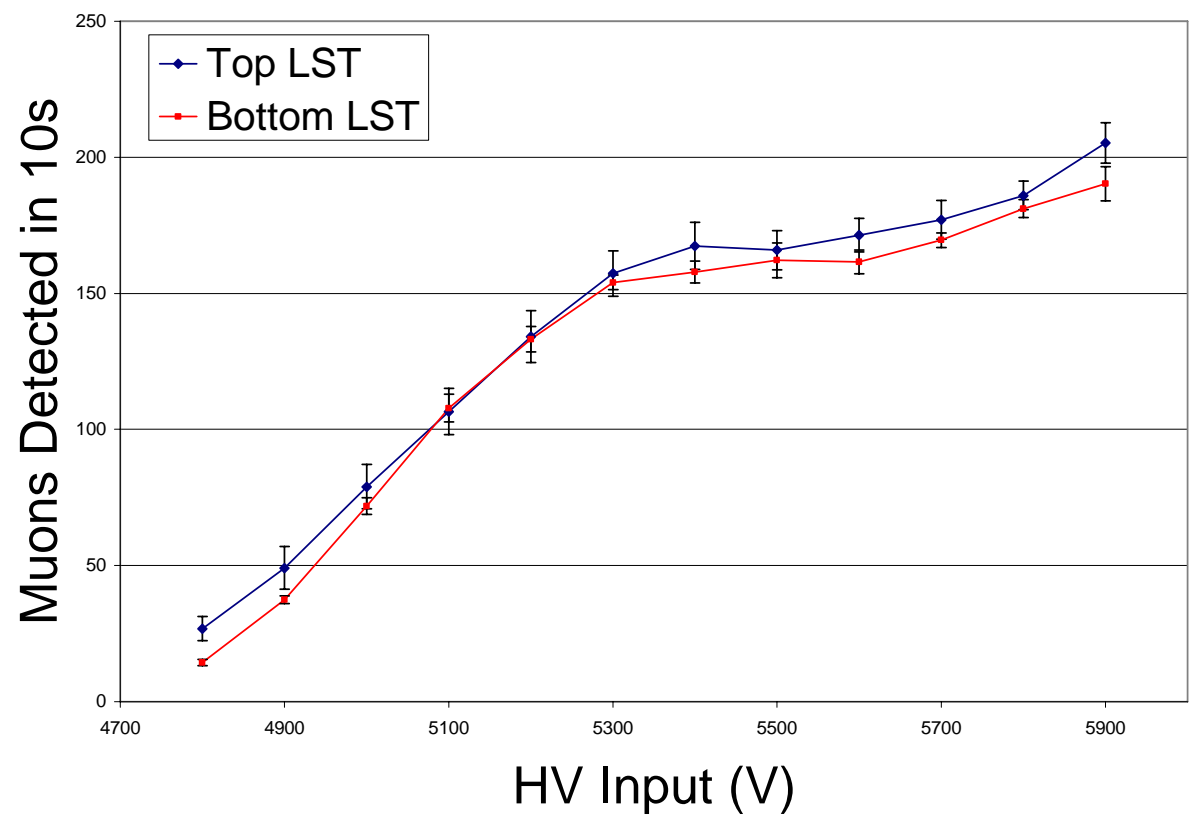

Figure 7. Singles rate tests for the top and bottom LSTs. Both curves show an edge at approximately $5300 \mathrm{~V}$ and plateau around $5500 \mathrm{~V}$.

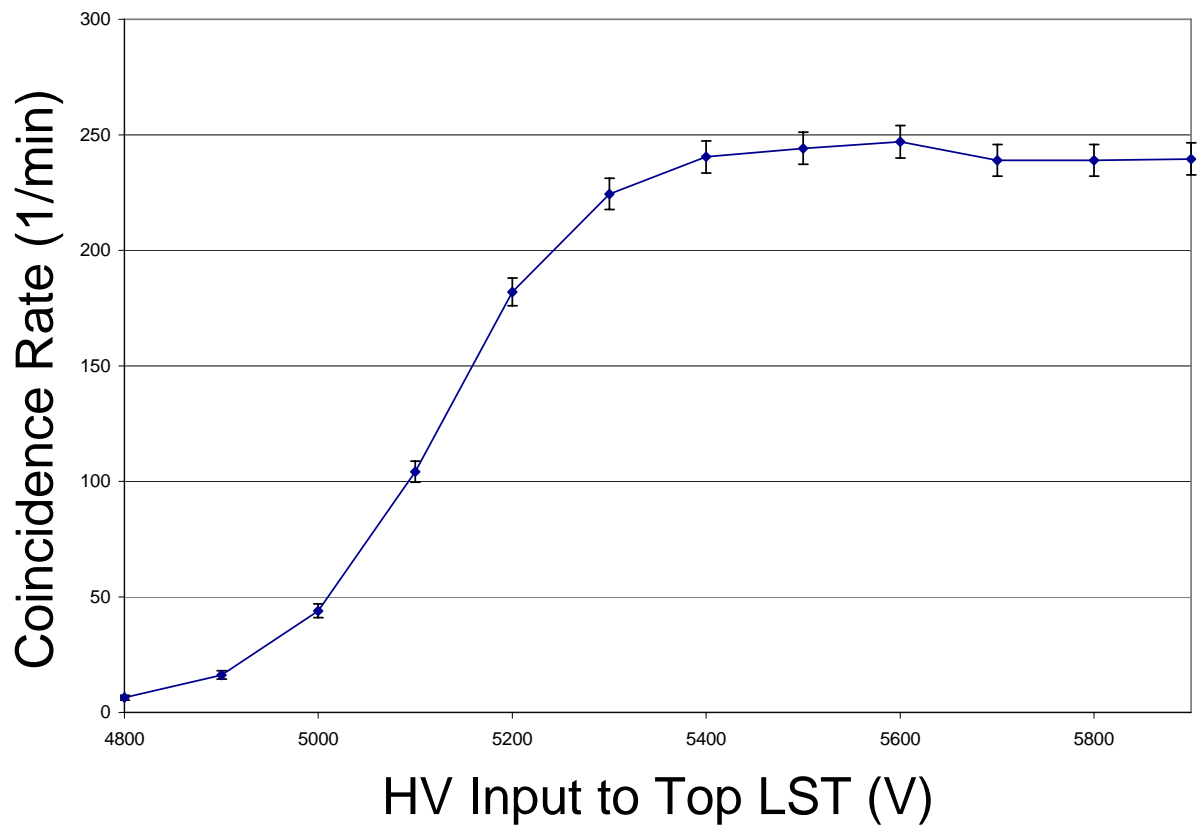

Figure 8. Coincidence rate as a function of the voltage supplied to the top LST as the bottom LST is help at optimal operating voltage, $5500 \mathrm{~V}$. 


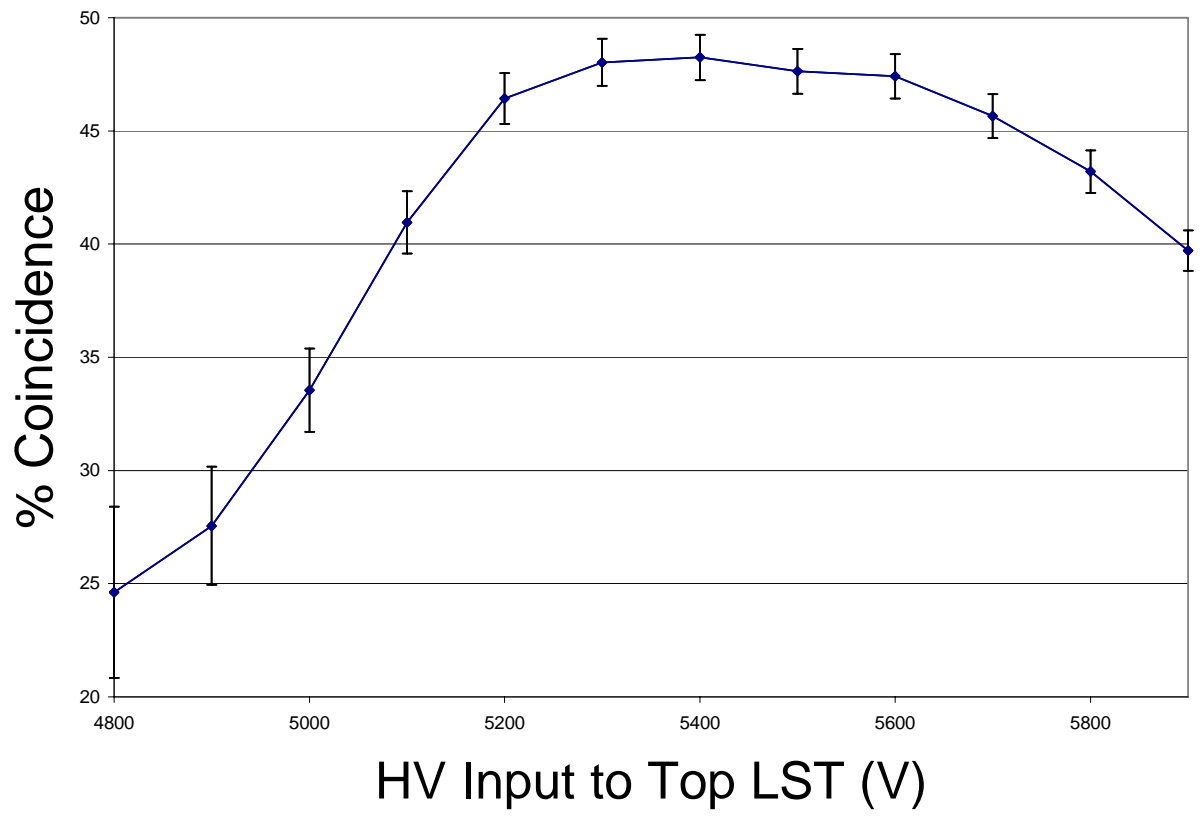

Figure 9. Percent coincidence as a function of the voltage supplied to the top LST as the bottom LST is held at optimal operating voltage, $5500 \mathrm{~V}$. 


\title{
Development of Nanofluidic Cells for Ultrafast X-ray Studies of Water
}

\author{
Melvin E. Irizarry \\ Office of Science, Science Undergraduate Laboratory Internship (SULI) \\ University of Puerto Rico, Mayagüez Campus \\ Stanford Linear Accelerator Center \\ Menlo Park, CA
}

August 18, 2006

Prepared in partial fulfillment of the requirements of the Office of Science, Department of Energy's Science Undergraduate Laboratory Internship under the direction of Aaron Lindenberg at the Stanford Linear Accelerator Center.

Participant:

Signature

Research Advisor:

\author{
Signature
}




\section{TABLE OF CONTENTS}

$\begin{array}{ll}\text { Abstract } & \text { ii }\end{array}$

$\begin{array}{ll}\text { Introduction } & 1\end{array}$

$\begin{array}{ll}\text { Materials and Methods } & 1\end{array}$

$\begin{array}{ll}\text { Results } & 2\end{array}$

$\begin{array}{ll}\text { Discussion and Conclusions } & 3\end{array}$

$\begin{array}{lr}\text { Acknowledgments } & 3\end{array}$

$\begin{array}{lc}\text { References } & 4\end{array}$

$\begin{array}{lr}\text { Figures } & 6\end{array}$

$\begin{array}{lr}\text { Tables } & 9\end{array}$ 


\begin{abstract}
Development of Nanofluidic Cells for Ultrafast X-ray Studies of Water. MELVIN E. IRIZARRY (University of Puerto Rico, Mayagüez Campus, Mayagüez, PR 00681) AARON LINDENBERG (Stanford Linear Accelerator Center, Menlo Park, CA 94025)
\end{abstract}

In order to study the molecular structure and dynamics of liquid water with soft x-ray probes, samples with nanoscale dimensions are needed. This paper describes a simple method for preparing nanofluidic water cells. The idea is to confine a thin layer of water between two silicon nitride windows. The windows are $1 \mathrm{~mm} \times 1 \mathrm{~mm}$ and $0.5 \mathrm{~mm} \times 0.5 \mathrm{~mm}$ in size and have a thickness of $150 \mathrm{~nm}$. The thickness of the water layer was measured experimentally by probing the infrared spectrum of water in the cells with a Fourier Transform InfraRed (FTIR) apparatus and from soft x-ray static measurements at the Advanced Light Source (ALS) at Lawrence Berkeley National Laboratory. Water layers ranging from $10 \mathrm{~nm}$ to more than $2 \mu \mathrm{m}$ were observed. Evidence for changes in the water structure compared to bulk water is observed in the ultrathin cells. 


\section{INTRODUCTION}

Molecular events like bond-breaking, vibrational excitations, or reorientational motion of molecules in the liquid state have a duration that ranges from femtoseconds to picoseconds. Ultrafast x-ray pulses are an ideal tool for probing the molecular regime of very short timescales and nano and atomic length-scales. Previous experiments have probed the electronic and structural properties of water using a variety of x-ray spectroscopic techniques $([1],[2]$, [3] and [4]). One of the central experimental obstacles in probing the dynamics of water with soft x-ray pulses in transmission is the design of a nanofluidic cell which confines a thin layer of water between two transparent windows in vacuum. These cells need to have a known and controllable thickness less than $500 \mathrm{~nm}$. A difficulty arises from the fluid nature of liquid water, making it complicated to obtain such ultrathin separations. The aim of this project was to develop a procedure for preparing such nanofluidic water cells.

\section{MATERIALS AND METHODS}

The nanofluidic cells were produced using lithographically designed $\mathrm{Si}_{3} \mathrm{~N}_{4}$ windows with a $500 \mathrm{~nm}$ photoresist spacer. Polystyrene nanospheres of $500 \mathrm{~nm}$ and $200 \mathrm{~nm}$ diameter were also used to define the sample thickness. A simple apparatus (Figure 1) was designed for reproducible sample manipulation. This apparatus allowed for the control and positioning of the windows using a micrometer stage. Water volumes ranging from 100-2000 nL were applied to the windows using a $2 \mu \mathrm{L}$ capacity syringe. Once the nanofluidic cell was assembled, it was stored in an aluminum sample holder (Figure 2) with a $5 \mathrm{~mm} \times 5 \mathrm{~mm}$ cavity and a 1 mm hole. A series of O-rings pushed the two windows together and sealed the cell in order to make it stable in vacuum. Infrared and x-ray spectroscopic measurements were used to characterize the samples. The infrared spectrum of the nanofluidic cells was obtained using a Fourier Transform InfraRed Spectrometer (FTIR) and compared to previous measurements of the absorption spectrum of liquid water by Bertie and Lan [5]. The measured absorbance 
$A$ is related to the absorption coefficient $\alpha$ by the relation

$$
A=\alpha x \log e,
$$

where $x$ is the sample thickness. The data was fitted using this relation and the sample thickness was extracted. Static soft x-rays transmission measurements of the samples were carried out at beamline 6.3.2 at the Advanced Light Source (ALS) at Lawrence Berkeley National Laboratory. The x-ray energy was scanned over a $50 \mathrm{eV}$ range around the Oxygen $\mathrm{K}$-edge and the transmission was measured using a photodiode. The recorded x-ray spectrum was compared to calculations using the Center for X-ray Optics (CXRO) Transmission Calculator [6].

\section{RESULTS}

Figure 3 shows the measured spectrum (blue line) and the data from Bertie and Lan (black squares). This sample used polystyrene spheres with $200 \mathrm{~nm}$ diameter and had a thickness estimate of $500 \mathrm{~nm}$. Intramolecular vibrational modes corresponding to the $\mathrm{O}-\mathrm{H}$ stretch and bending modes are clearly resolved at $3440 \mathrm{~cm}^{-1}$ and $1650 \mathrm{~cm}^{-1}$ respectively. These peaks were used to extract the sample thickness as described above. The silicon nitride stretching mode is also observed close to $850 \mathrm{~cm}^{-1}$. Table 1 presents a list of the sample thicknesses that were measured with the FTIR. This thickness ranged from $150 \mathrm{~nm}$ to less than $2 \mu \mathrm{m}$.

Figure 4 is an example of the data obtained by x-ray transmission measurements (blue) with the fit calculated by the CXRO program (red), which does not include near-edge effects and thus does not provide a good fit very close to the Oxygen K-edge. The overall jump in transmission was used to extract the thickness. The pre-edge peak, around $535 \mathrm{eV}$, is well-resolved. Thicker samples were observed (with thickness on the order of 1 micron) as well as ultrathin layers (less than $10 \mathrm{~nm}$ ). Table 2 lists the extracted sample thicknesses for x-ray measurements. 


\section{DISCUSSION AND CONCLUSIONS}

Despite careful control of the sample preparation process, measured thicknesses varied significantly. Additional research is needed to obtain controllable separations. Possible directions for future investigation include control of the angle of approach of the two $\mathrm{Si}_{3} \mathrm{~N}_{4}$ windows as well as variations in the shape of the photoresist spacer to incorporate a small channel for the water to flow in and out. Simulation of the capillary forces that influence the adhesion of the windows could also lead towards improvements in the sample assembly.

Analysis of the ultrathin $(<50 \mathrm{~nm})$ samples indicates surprising changes in the absorption spectrum compared to the known liquid water absorption spectrum [7]. Figure 5 shows a superposition of x-ray transmission spectra from different samples. The bigger the change in transmission, the thicker the sample. A shift toward lower energy of about $1 \mathrm{eV}$ is observed

as the sample thickness decreases. A similar shift towards lower energy is observed when comparing the spectrum of ice to liquid water [7]. This shift can then be interpreted as indicative of a change to a more ice-like, tetrahedral structure upon confinement. This means that a very thin layer of liquid water behaves more like solid ice. The ability to produce ultrathin samples enables future experiments probing the structure and dynamics of confined liquids [8]. These nanofluidic cells will also allow for pump-probe experiments in which a femtosecond laser is used to break bonds in the liquid state and the resulting dynamics are probed using ultrafast x-ray pulses.

\section{ACKNOWLEDGMENTS}

This research was carried out in the Stanford Linear Accelerator Center in the summer of 2006. I would like to thank the Department of Energy, Office of Science for providing me the chance to participate in the SULI program and learn more about nature's most interesting behaviors. Special thanks to Aaron Lindenberg for his kind mentoring and advices. I also thank the Department of Chemistry at Stanford University for the FTIR support and 
Lawrence Berkeley National Laboratory for beamtime at ALS.

\section{REFERENCES}

[1] B. X. Yang and J. Kirz, "Extended x-ray-absorption fine structure of liquid water," Phys. Rev. B, vol. 36, no. 2, pp. 1361-1364, Jul 1987.

[2] K. Wilson, B. Rude, T. Catalano, R. Schaller, J. Tobin, D. Co, and R. Saykally, "X-ray spectroscopy of liquid water microjets," Journal of Physical Chemistry B, vol. 105, no. 17, pp. 3346-3349, 2001. [Online]. Available: http://dx.doi.org/10.1021/jp010132u

[3] J. D. Smith, C. D. Cappa, K. R. Wilson, B. M. Messer, R. C. Cohen, and R. J. Saykally, "Energetics of Hydrogen Bond Network Rearrangements in Liquid Water," Science, vol. 306, no. 5697, pp. 851-853, 2004. [Online]. Available: http://www.sciencemag.org/cgi/content/abstract/306/5697/851

[4] L.-A. Näslund, J. Luning, Y. Ufuktepe, H. Ogasawara, P. Wernet, U. Bergmann, L. Pettersson, and A. Nilsson, "X-ray absorption spectroscopy measurements of liquid water," Journal of Physical Chemistry B, vol. 109, no. 28, pp. 13835-13839, 2005. [Online]. Available: http://dx.doi.org/10.1021/jp052046q

[5] J. E. Bertie and Z. Lan, "Infrared Intensities of Liquids XX: The Intensity of the $\mathrm{OH}$ Stretching Band of Liquid Water Revisited, and the Best Current Values of the Optical Constants of $\mathrm{H}_{2} \mathrm{O}(\mathrm{l})$ at $25^{\circ} \mathrm{C}$ between 15,000 and $1 \mathrm{~cm}^{-1}$," Applied Spectroscopy, vol. 50, no. 8, pp. 1047-1057, 1996.

[6] B. L. Henke, E. M. Gullikson, and J. C. Davis, "X-ray interactions: photoabsorption, scattering, transmission, and reflection at $e=50-30000 \mathrm{eV}, z=1-92$," Atomic Data and Nuclear Data Tables, vol. 54, no. 2, pp. 181 - 342, 1993. 
[7] P. Wernet, D. Nordlund, U. Bergmann, M. Cavalleri, M. Odelius, H. Ogasawara, L. A. Näslund, T. K. Hirsch, L. Ojamae, P. Glatzel, L. G. M. Pettersson, and A. Nilsson, "The structure of the first coordination shell in liquid water," Science, vol. 304, no. 5673, pp. 995-999, 2004. [Online]. Available: http: //www.sciencemag.org/cgi/content/abstract/304/5673/995

[8] S. Granick, "Motions and relaxations of confined liquids," Science, vol. 253, pp. 13741379, Sept. 1991. 


\section{FIGURES}

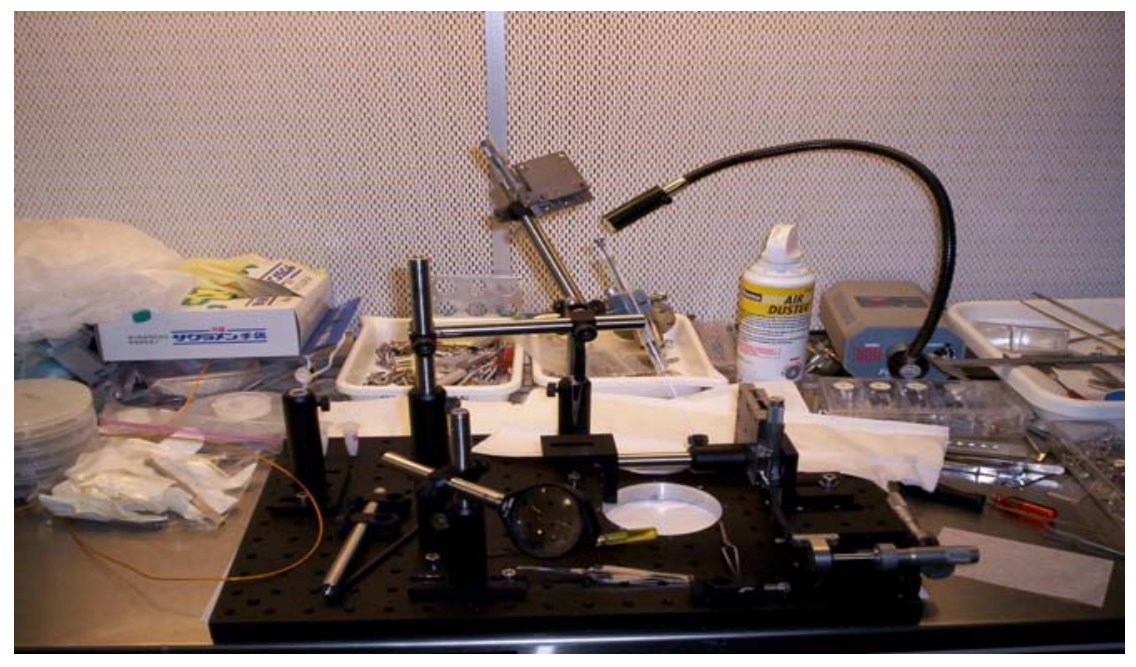

Figure 1: The device developed for sample assembly. It consisted of tweezers, a $2 \mu \mathrm{L}$ sysringe, and micrometer stages for precise manipulation of silicon nitride windows.

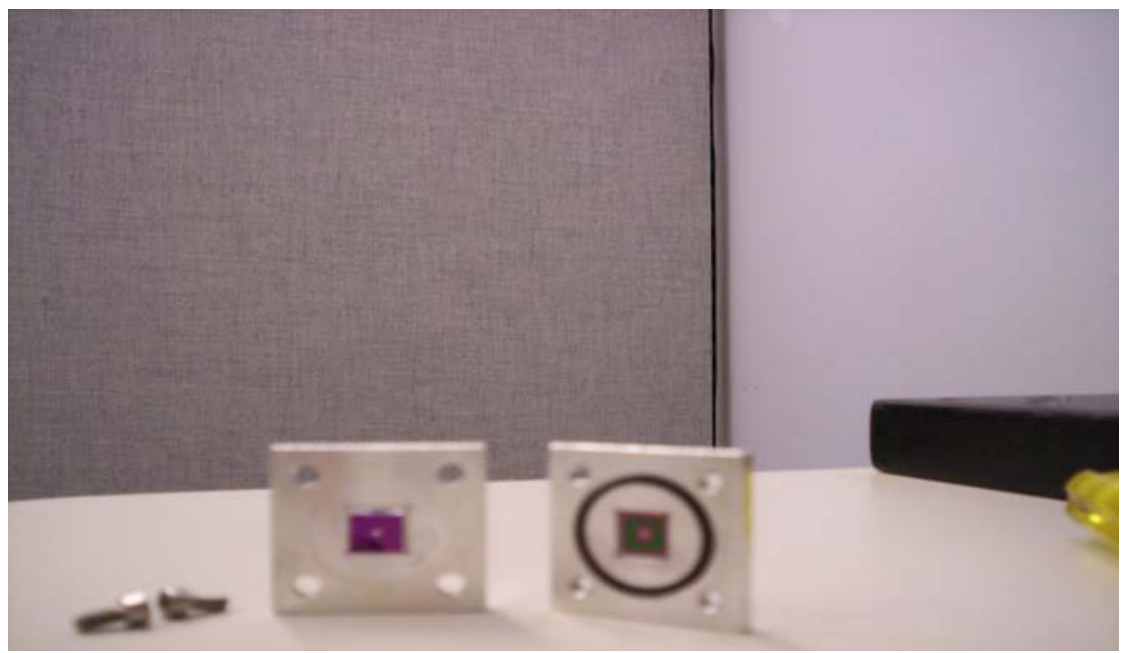

Figure 2: Aluminum sample holder used to store the nanofluidic cell during experiments. 


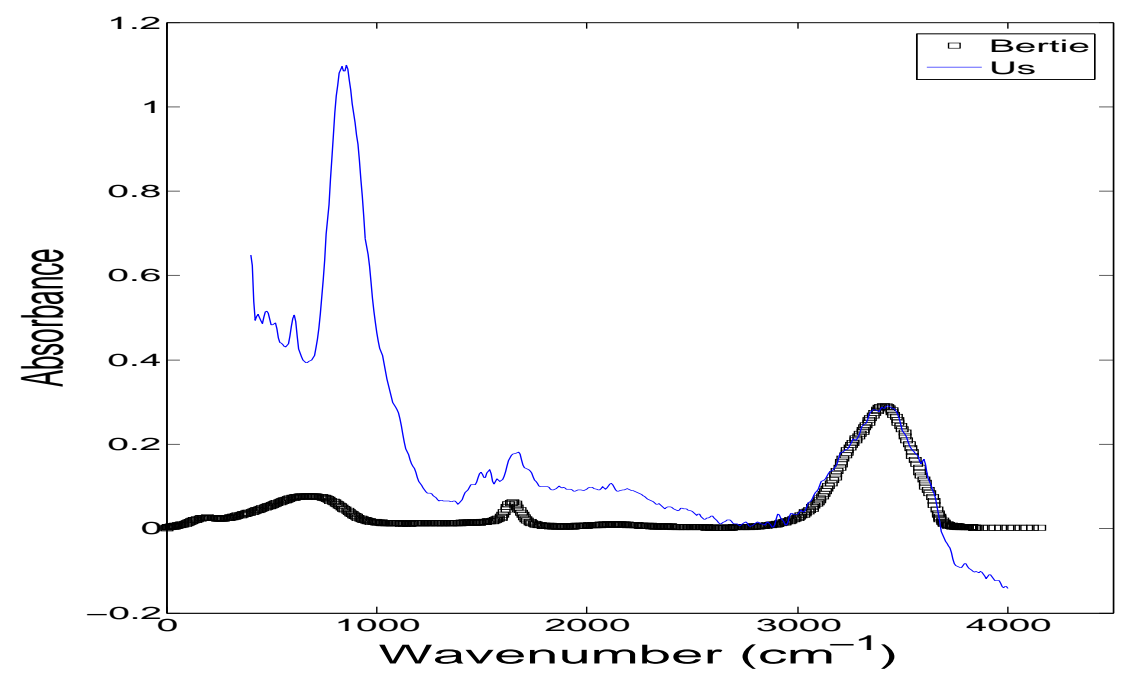

Figure 3: Infrared spectrum of nanofluidic cell with polystyrene spheres. The black squares is the data from Bertie and Lan. O-H symmetric stretching and $\mathrm{H}$ bending modes are observed and used for the fitting and determination of sample thickness.

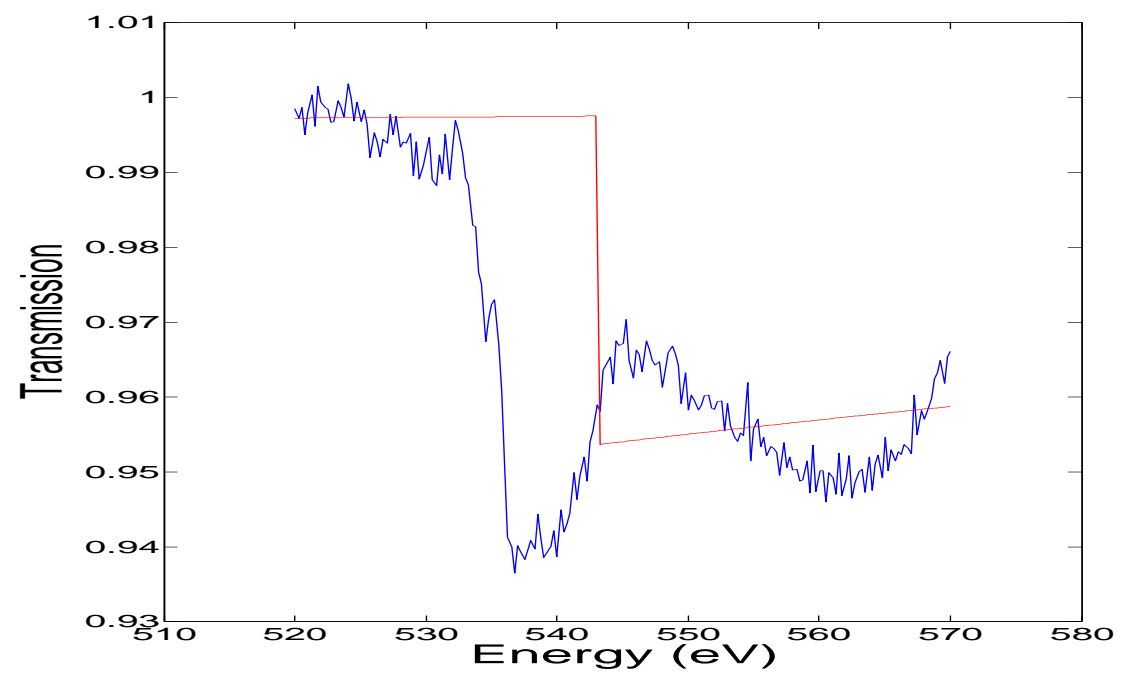

Figure 4: X-ray transmission spectra measured (blue) and calculated with CXRO program (red). Fit corresponds to a $25 \mathrm{~nm}$ thick liquid layer. See text for details of fitting procedure. 


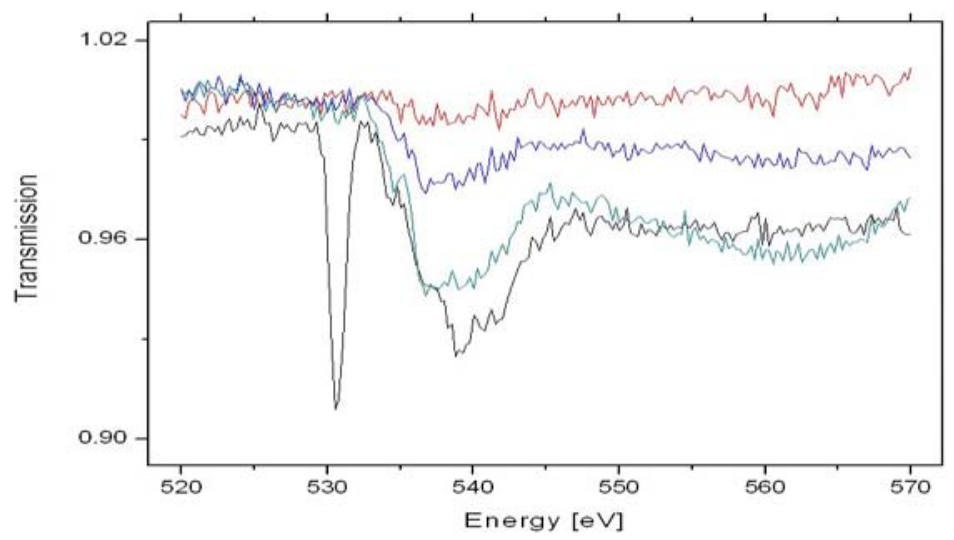

Figure 5: Superposition of x-ray spectrum from different samples. A shift in the main absorption peak towards lower energy as sample thickness decreases is observed. This can be interpreted as an indication of a change from a liquid to a more ice-like molecular structure. 


\section{TABLES}

\begin{tabular}{|c|c|}
\hline Water & Polystyrene spheres \\
\hline $1000 \mathrm{~nm}$ & $1010 \mathrm{~nm}$ \\
\hline $450 \mathrm{~nm}$ & $520 \mathrm{~nm}$ \\
\hline $220 \mathrm{~nm}$ & $1750 \mathrm{~nm}$ \\
\hline $145 \mathrm{~nm}$ & $1500 \mathrm{~nm}$ \\
\hline $150 \mathrm{~nm}$ & $500 \mathrm{~nm}$ \\
\hline & $1800 \mathrm{~nm}$ \\
\hline
\end{tabular}

Table 1: Sample thickness (FTIR)

\begin{tabular}{|c|c|}
\hline Water & Polystyrene spheres \\
\hline $15 \mathrm{~nm}$ & $1 \mathrm{~nm}$ \\
\hline $5 \mathrm{~nm}$ & $10 \mathrm{~nm}$ \\
\hline $15 \mathrm{~nm}$ & $17 \mathrm{~nm}$ \\
\hline & 17 \\
\hline & $25 \mathrm{~nm}$ \\
\hline
\end{tabular}

Table 2: Ultrathin samples observed with the x-ray transmission experiments 


\title{
MONITORING DISPLAYS FOR GLAST
}

\author{
Building ISOC Status Displays for the Large Area Telescope aboard the Gamma Ray \\ Large Area Space Telescope (GLAST) Observatory \\ Christina Ketchum \\ Office of Science, SULI Program \\ Stanford University, California \\ Stanford Linear Accelerator Center \\ Menlo Park, California
}

August 2, 2006

Prepared in partial fulfillment of the requirements of the Office of Science, U.S. Department of Energy Science Undergraduate Laboratory Internship (SULI) Program under the direction of Rob Cameron in the Kavli Institute for Particle Astrophysics and Cosmology Division of the Stanford Linear Accelerator Center.

Participant:

Signature

Research Advisor:

Signature 


\section{Table of Contents}

$\begin{array}{lc}\text { Abstract } & 3 \\ \text { Introduction } & 4 \\ \text { Materials and Methods } & 5 \\ \text { Results } & 8 \\ \text { Discussion and Conclusions } & 9 \\ \text { Acknowledgements } & 10 \\ \text { References } & 10 \\ \text { Figures } & 11\end{array}$




\begin{abstract}
Building ISOC Status Displays for the Large Area Telescope aboard the Gamma Ray Large Area Space Telescope (GLAST) Observatory. CHRISTINA KETCHUM (Lewis and Clark College, Portland, OR 97219) ROB CAMERON (Stanford Linear Accelerator Center, Menlo Park, CA 94025).
\end{abstract}

In September 2007 the Gamma Ray Large Area Space Telescope (GLAST) is scheduled to launch aboard a Delta II rocket in order to put two high-energy gamma-ray detectors, the Large Area Telescope (LAT) and the GLAST Burst Monitor (GBM) into low earth orbit. The Instrument Science Operations Center (ISOC) at SLAC is responsible for the LAT operations for the duration of the mission, and will therefore build an operations center including a monitoring station at SLAC to inform operations staff and visitors of the status of the LAT instrument and GLAST. This monitoring station is to include sky maps showing the location of GLAST in its orbit as well as the LAT's projected field of view on the sky containing known gamma-ray sources. The display also requires a world map showing the locations of GLAST and three Tracking and Data Relay Satellites (TDRS) relative to the ground, their trail lines, and “footprint” circles indicating the range of communications for each satellite. The final display will also include a space view showing the orbiting and pointing information of GLAST and the TDRS satellites.

In order to build the displays the astronomy programs Xephem, DS9, SatTrack, and STK were employed to model the position of GLAST and pointing information of the LAT instrument, and the programming utilities Python and Cron were used in Unix to obtain updated 
information from database and load them into the programs at regular intervals. Through these methods the indicated displays were created and combined to produce a monitoring display for the LAT and GLAST.

\section{INTRODUCTION}

Gamma-rays are the most energetic rays in the electromagnetic energy spectrum, with energies beginning at $40 \mathrm{keV}$. Because of the amount of energy required to produce such photons, gamma-rays are necessarily created in some of the most extreme conditions in the universe. Energetic phenomena such as pulsars, collapsing binary neutron stars, hypernovae, and active galactic nuclei have the capability of accelerating charged particles to relativistic speeds. The radiation emitted from these particles is Doppler boosted into the gamma-ray energy spectrum, which effectively beams gamma-rays across the universe. There are two classifications of gamma-ray emission. The first of which are called gamma-ray bursts (GRBs), which last from 1 to 100 seconds. An average of one GRB is detected every day, indicating that they are a fairly common event. The second type is long-term variable gamma-ray emission, which last from days to weeks. These features of the sky are constantly changing as new cosmic events trigger gamma-ray production. Currently catalogs of gamma ray sources, such as the 3EG catalog that was produced from data taken by the EGRET (Energetic Gamma-ray Experiment Telescope) have relatively few objects. The 3EG catalog has only 271 sources and of these, 170 are unidentified. It is predicted that with the use of the next generation instruments such as the LAT on GLAST these sources can be identified, and perhaps as many as 10,000 new gamma-ray sources will be discovered. More information can also be gathered about the extreme conditions 
necessary to produce gamma-rays by detecting and cataloging gamma-ray emission in the universe.

The Gamma-ray Large Area Space Telescope (GLAST) satellite houses two different forms of gamma-ray detectors, the Large Area Telescope (LAT) shown in Figure 1, and the GLAST Burst Monitor (GBM) to detect high energy gamma-ray emission and short powerful bursts of lower energy gamma-rays respectively. SLAC has managed the construction of the LAT and will be responsible for the operations of the LAT while it is in orbit and will therefore require status displays on the position, attitude, contact times and durations, and potential gamma-ray sources that the LAT will be viewing.

In order to communicate this information to the staff working with the LAT as well as visitors, two maps must be developed to track GLAST through its 96 minute orbit around the Earth and to plot its field of view of the sky. The main objective is to control such programs with the Unix timing application "cron" so that the information used to construct the map will be obtained weekly and updated daily on each display with positional information distributed by NASA (National Aeronautics and Space Administration). These processes will be automated so that no user input is required and so run continuously for the duration of the GLAST mission (from 5 to 10 years). GLAST orbital ephemerides and contact schedules will also be regularly obtained from NASA distributions, interpreted, and fed into a usable display. Many different satellite tracking programs can be used to create the Earth view with a satellite overlay and the sky field of view map. It is necessary to select which satellite tracking programs provide the most accessible and accurate displays and to combine these individual maps into a coherent operations display.

\section{MATERIALS AND METHODS}


In order to identify the most useful satellite tracking and astronomy programs for the display, a number of different programs were investigated. After some preliminary research on the capabilities of software programs there were three that seemed to be helpful. Xephem, which is software available for free on the internet at http://www.clearskyinstitute.com/xephem/, has numerous capabilities, including that of a sky visualization, complete with ground station masks (simulated horizon lines), constellation projections, and the ability to load external data catalogs. Accordingly Xephem was used for simulating a local sky view and tracking GLAST as it passes overhead. The program STK was used for the ground tracking map based on the quality of its maps and images, and the ease with which satellites can be added to the display and modified. SatTrack was selected to create a world view display with LAT pointing information because of its ability to produce detailed space visualizations. And finally the program DS9 was used to create an Aitoff projection all sky view map with the LAT field of view.

The next step was to enable these four programs to use external data. STK and Xephem require satellite information in Two Line Element (TLE) [1] format and use their own propagators to extract orbital information from those elements. However, the positional information that ISOC receives from NASA is in the form of Ephemerides, which includes the $\mathrm{x}$, $\mathrm{y}$, and $\mathrm{z}$ coordinates at one minute time intervals of the satellite on an earth centered inertial (ECI) coordinate system. Due to a difference in coordinate systems between the provided data and the STK required format it was necessary to use TLEs in order to plot the orbits of satellites in STK. The information regarding GLAST satellite pointing is also provided by NASA in a file called the Preliminary Science Timeline. This file contains extraneous data that was not required for the pointing location of the satellite, so the file had to be parsed using a program written in Python to extract the slewing positions of GLAST. Then the resulting pointing information was 
saved as an attitude file as a part of SatTrack. Two other SatTrack files, the object data catalog and the defaults data catalog, had to be altered as well to direct SatTrack to read the attitude data from file. The Tracking and Data Relay Satellite System (TDRSS) which is a network of geostationary communications satellites will be relaying data transmitted from GLAST to the ground stations. The TDRSS Forecast Schedule was used to obtain a list of predicted ground station contact times for GLAST.

The GLAST and TDRSS Ephemerides files and the TDRSS Forecast Schedule are uploaded weekly to a database kept on the SLAC server, while the Preliminary Science Timeline is also updated weekly but remains in file format. The program Cron was used to automate their retrieval, formatting, and usage, so that every week at a regular time of night all the required information regarding GLAST and TDRSS would be updated and then loaded into STK, Xephem, SatTrack, and DS9.

To create the full sky map the program DS9 was used. First the program 'gtbin' was used to produce a Flexible Image Transport System (FITS) file that simulates what the gammaray sky would look like as a background sky map or after launch, load actual GLAST data into the image. This image was loaded into DS9 then overlaid with a galactic coordinate system in an Aitoff style projection. The gamma-ray source data obtained by EGRET aboard the Compton satellite was also run through "gtbin" in order to create a region file for DS9. The resulting file was then loaded into DS9 to create small circles surrounding each known gamma-ray source in the sky. Then the program 'gtorbsim' was used to model real-time satellite pointing information with the Right Ascension (RA) and Declination (Dec) position of the boresight of the LAT. To define the LAT field of view a program function called "Region" of DS9 was utilized. The RA and Dec of the boresight were then run through a program created by Mr. James Chiang to 
output an array of points defining the circle of the field of view. These points, when placed on an Aitoff projection map resulted in circles that deformed as the coordinate system stretched at the edges of the map to represent the field of view. A python program was then written to smooth out the shape as it approached the edges of the map and eliminate extraneous line creation that resulted when the field of view was wrapped from the left to the right side of the map. The image including the simulated gamma-ray sky, known gamma-ray sources, and the field of view of the LAT was then driven through the XPA interface so that DS9 commands can be issued from the command line of the Unix machine and eventually regulated by Cron.

\section{RESULTS}

The final product achieved through the use of Xephem, SatTrack, STK, and DS9 was very similar to the goal initially set. Through STK a ground map as in Figure 2 was produced that displays the real-time locations of GLAST, TDRS-East, TDRS-West, and TDRS-Z in addition to outlining the South Atlantic Anomaly Region (SAAR). The SAAR is the area above the Earth that the ionic particles trapped in the Van Allen radiation belt come closest to the Earth's surface. The interference that this causes with the LAT is so great that no data will be taken during the time that GLAST is in the SAAR. [2] All satellites have "footprints" of their available contact ranges as well as leading and following sub-satellite position trails. In SatTrack a real-time pointing and location representation shown in Figure 3 was developed. The satellites are represented as wire cubes with different colored lines extending from their bodies to indicate their $\mathrm{x}, \mathrm{y}$, and $\mathrm{z}$ axis. The $\mathrm{z}$-axis or boresight of GLAST, indicated by a red line, moves according to the slewing information provided by NASA. In Xephem a sky view as in Figure 4 was created to indicate the position of GLAST with respect to an observer at SLAC. The path of GLAST is tracked across the sky and there are time stamps at the beginning and end points of the 
current pass. In DS9 a display was created represented in Figure 5 showing the field of view of the LAT on the gamma-ray sky, as well as indicating which known gamma-ray sources are within the field of view.

\section{DISCUSSION AND CONCLUSION}

The final step of the project was to combine the displays created with Xephem, STK, SatTrack, and DS9 on to one monitor for easy accessibility. The four displays cover enough view points for the location and attitude of the LAT to be clearly represented.

Throughout the development of the displays for GLAST and the LAT, many requirements had to be changed. Some early goals were discarded due to limitations of the software. Initially the ground display on STK was to incorporate contact times as well as satellite positions, trails and footprints. The portions of GLAST's trail that were available for communications link to one of the TDRSS satellites would be highlighted. However, with only the free version of STK the feature that allowed such a function was disenabled although available in the full version of STK. The world view supplied by SatTrack is only available in the purchased version. For future satellite tracking projects STK in its full purchased version would be useful. STK is technically and computationally less capable than SatTrack, but the degree of ease with which it can be used is far superior. The graphics supplied by the program STK are also of a better quality than those possessed by SatTrack. STK has many different options with which to represent the world map including an image of the Earth created from actual photos taken from space, while SatTrack only represents the Earth with continent outlines and an optional latitude and longitude grid. 
Other boundaries imposed on this project were temporal in nature. All of the utilized data currently supplied to ISOC about GLAST is only modeled data because GLAST is not currently in orbit. Specifically the Preliminary Science Timeline is only available currently as an example file, which contains no actual attitude information, only the correct formatting for such a document. Any inaccuracies which may arise in the parsing and application of this file can only be remedied once GLAST is in orbit and actual data is being supplied.

A future possibility for improvement of the display would be to include another Aitoff sky view map. With the second map the actual gamma-ray data taken by LAT over the last 24 hours could be displayed and compiled into an accelerated movie of the previous day's gammaray detection. This would provide a way for visitors to the LAT operations center to directly view the product of the GLAST project and have a physical sense of what the project is actually accomplishing.

\section{ACKNOWLEDGEMENTS}

This research was conducted at the Stanford Linear Accelerator Center. I thank the U. S. Department of Energy, Office of Science for giving me the opportunity to participate in the SULI program and the chance to have an incredible learning experience. Special thanks go to my mentor Rob Cameron for his knowledge, patience, and humor. I would also like to thank Steve Culp for making sure I had access to all the data I needed, Rodney Wong for handling numerous computer issues, and James Chiang for being a constant support in developing the Aitoff projections.

\section{REFERENCES}

[1] Dr. T.S. Kelso, CelesTrak, \{http://www.celestrak.com $\}$, May 2006. 
[2] "Space Flight Questions and Answers", NASA,

\{http://www.hq.nasa.gov/osf/qanda2.html\}, June 2004.

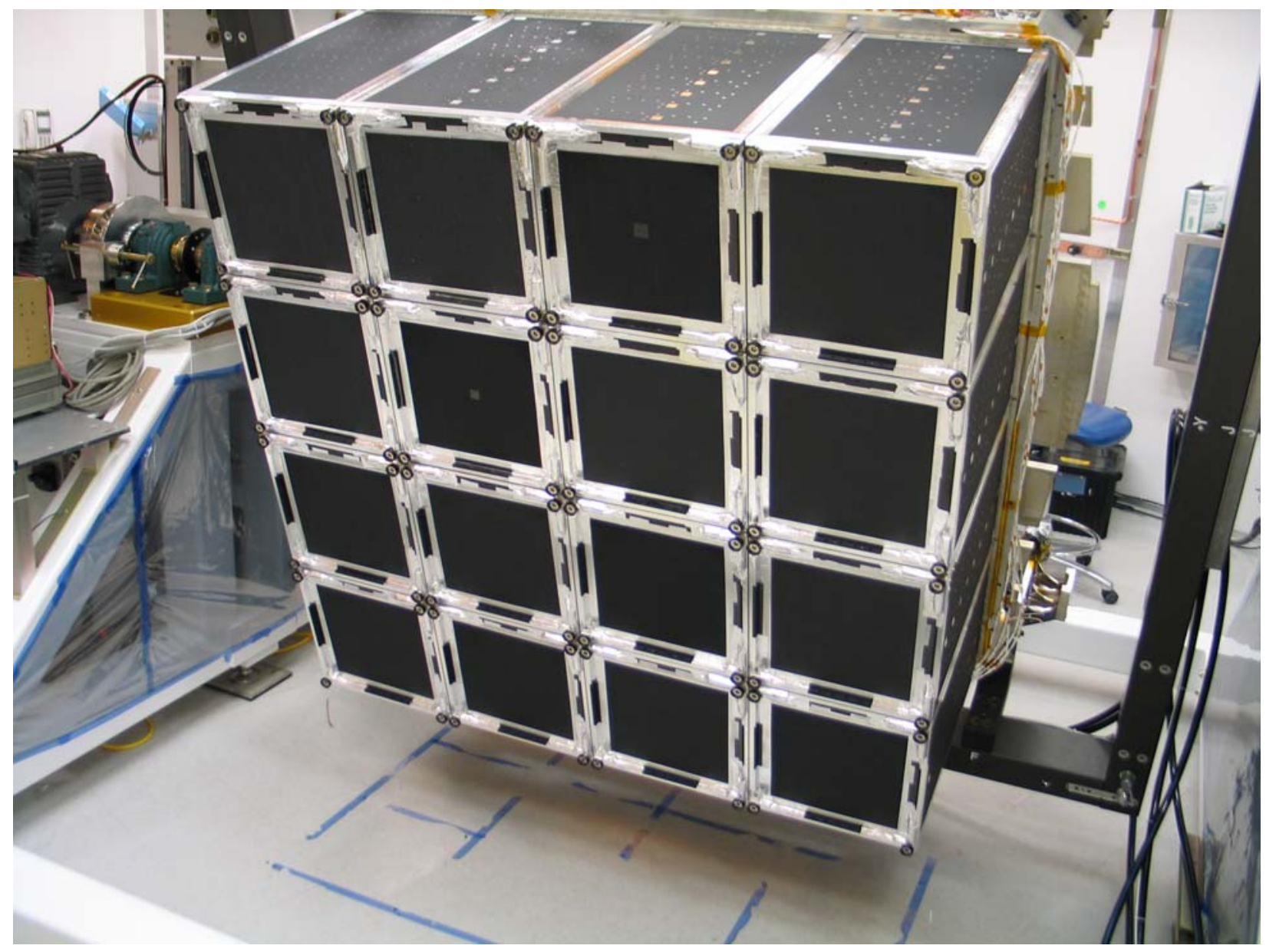

Figure 1. A photo of the Large Area Telescope (LAT) after being built at SLAC. 


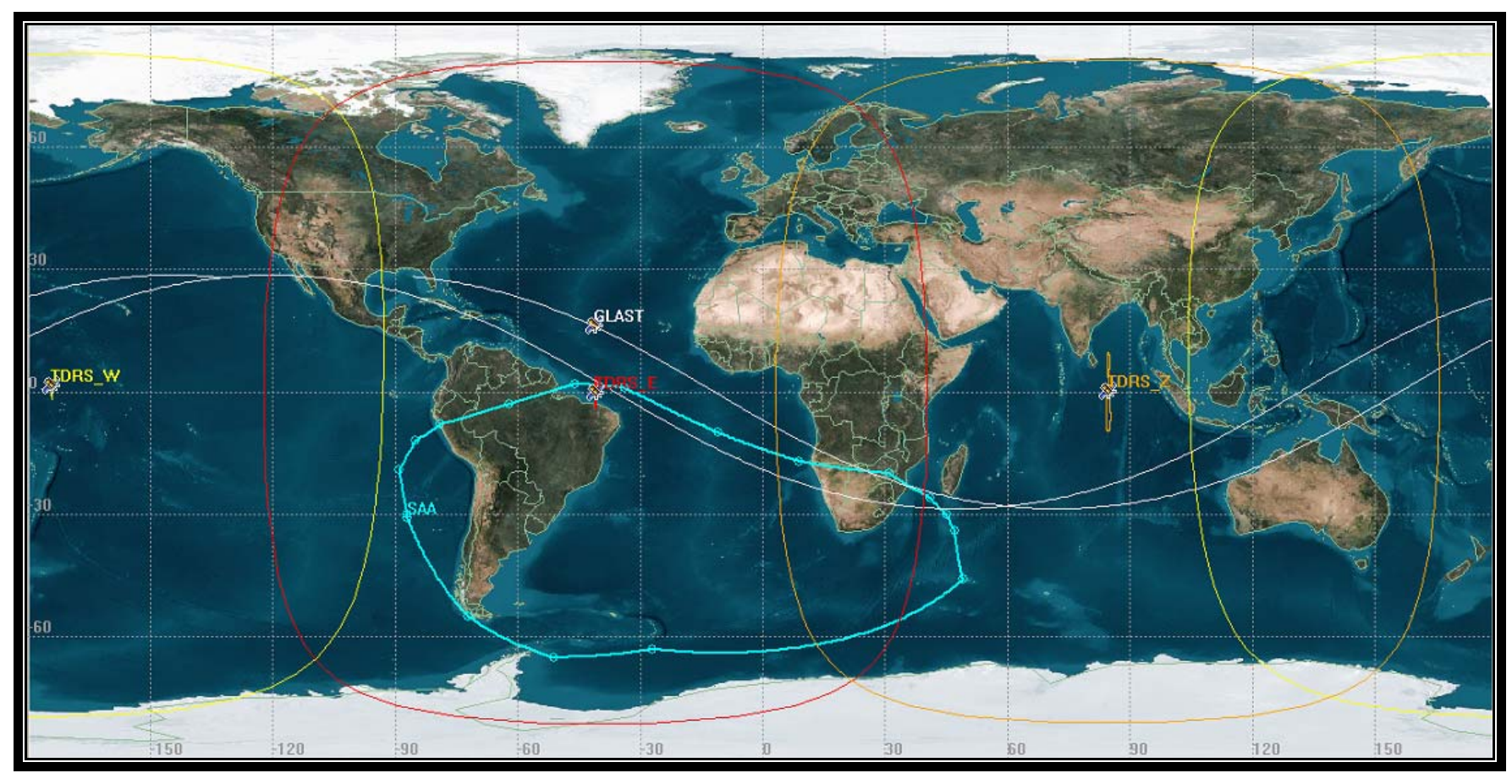

Figure 2. Ground view map of Earth including the GLAST, TDRS-East, TDRS-West, and TDRS-Z satellites, and the South Atlantic Anomaly (SAA) Region created with STK. 


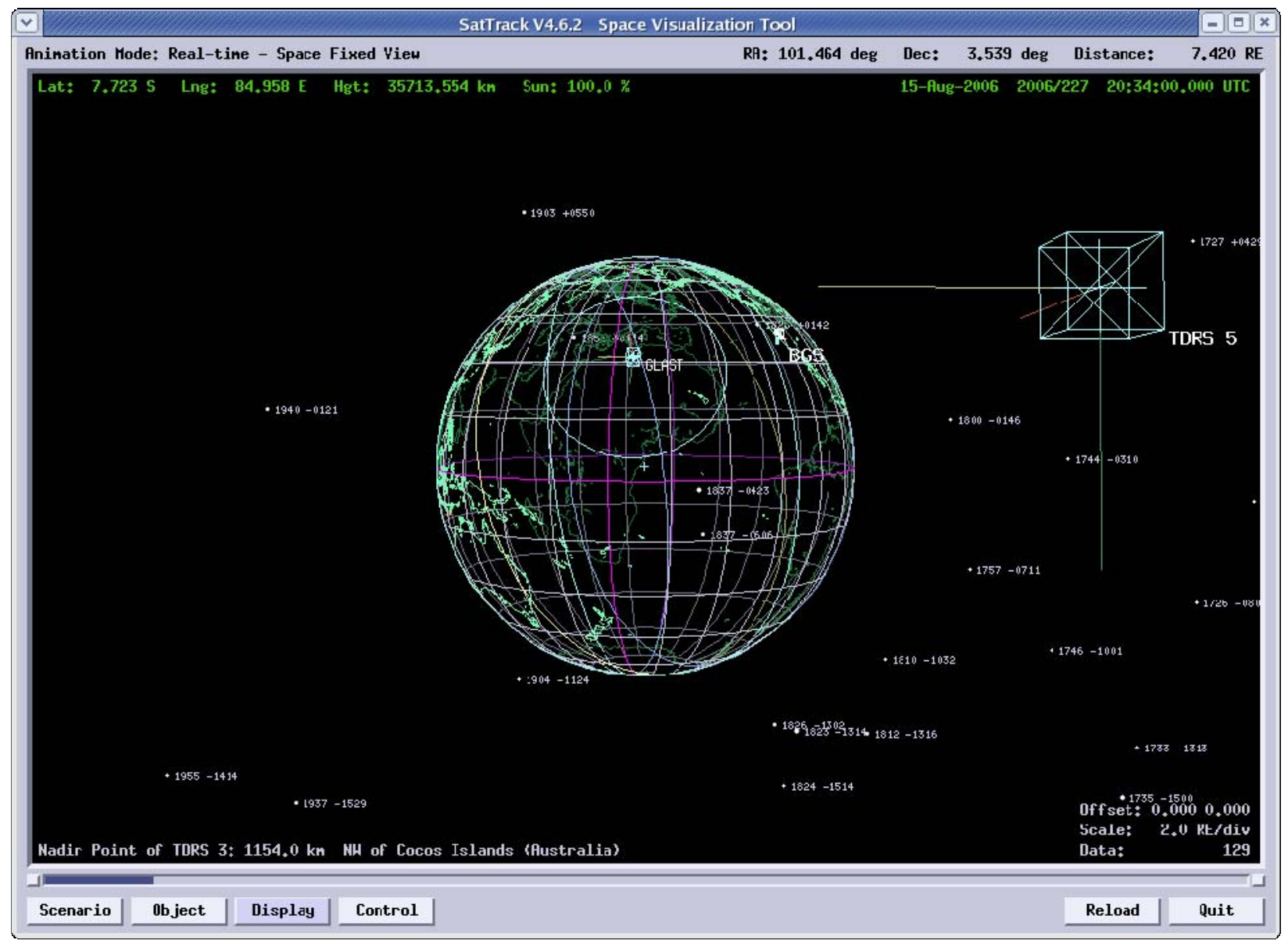

Figure 3. Display created in SatTrack showing the Earth with GLAST orbiting close above it, the TDRS 5 satellite in the foreground, and a number of gamma-ray sources in the sky. 


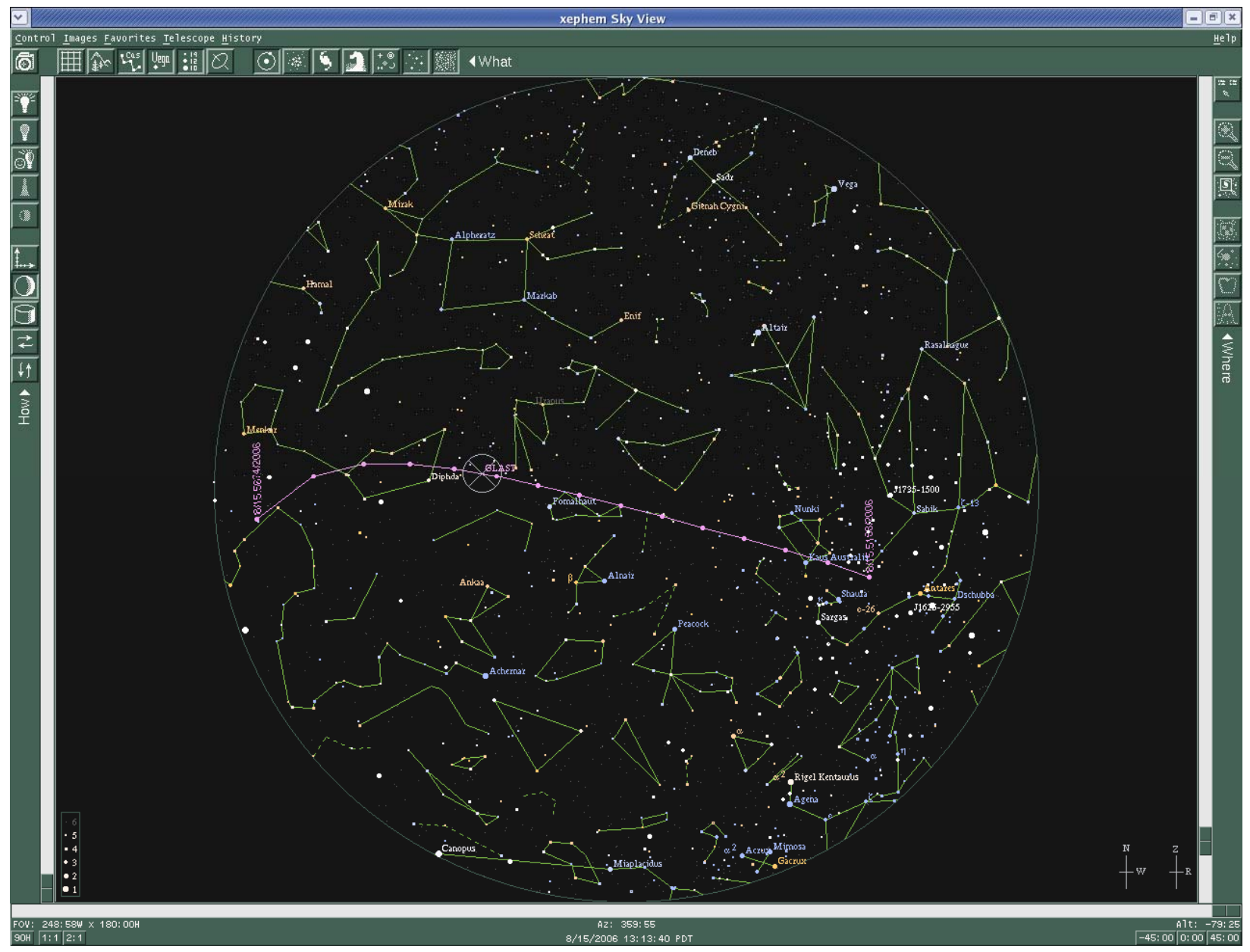

Figure 4. Sky view map showing the local stars and constellations with the path of the GLAST satellite created in Xephem. 


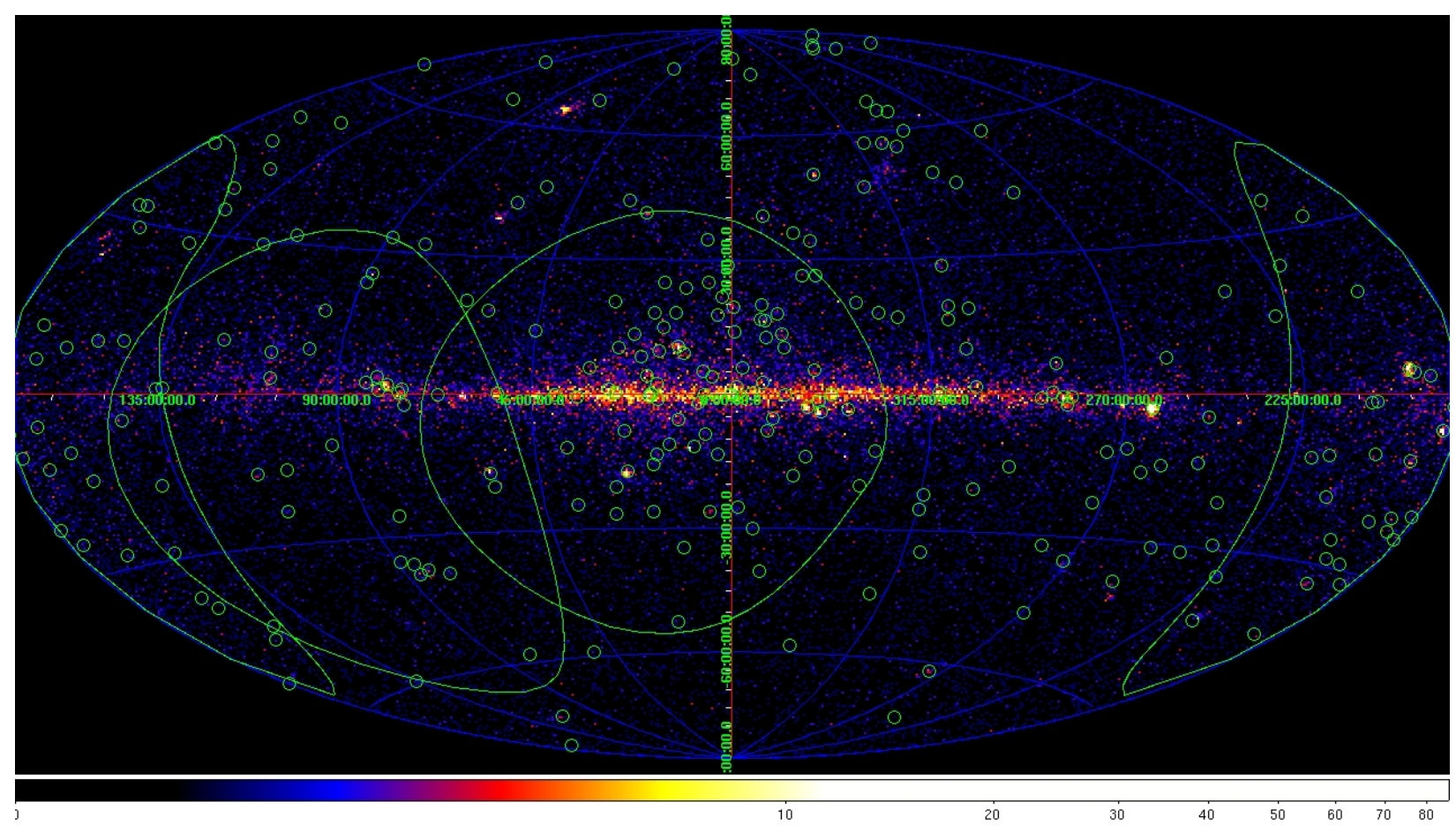

Figure 5. An Aitoff style projection of the simulated gamma-ray sky with circle indicators of known gamma-ray sources and deformed circles indicating the LAT's projected field of view. 


\title{
Upgrading the Digital Electronics of the PEP-II Bunch Current Monitors at the Stanford Linear Accelerator Center
}

\author{
Josh Kline \\ Office of Science, Science Undergraduate Laboratory Internship (SULI) \\ Sacramento State University \\ Stanford Linear Accelerator Center \\ Stanford, CA
}

August 22, 2006

Prepared in partial fulfillment of the requirements of the Office of Science, Department of Energy's Science Undergraduate Laboratory Internship under the direction of Alan Fisher at the Accelerator Department, Stanford Linear Accelerator Center.

Participant:

Signature

Research Advisor:

\author{
Signature
}




\section{TABLE OF CONTENTS}

$\begin{array}{ll}\text { Abstract } & \text { ii }\end{array}$

$\begin{array}{ll}\text { Introduction } & 1\end{array}$

Materials and Methods $\quad 5$

Discussion and Conclusions $\quad 5$

$\begin{array}{lr}\text { References } & 6\end{array}$ 


\begin{abstract}
Upgrading the Digital Electronics of the PEP-II Bunch Current Monitors at the Stanford Linear Accelerator Center. JOSH KLINE (Sacramento State University, Sacramento, CA) ALAN FISHER (Accelerator Department, Stanford Linear Accelerator Center, Stanford, CA 94025)
\end{abstract}

The testing of the upgrade prototype for the bunch current monitors (BCMs) in the PEP-II storage rings at the Stanford Linear Accelerator Center (SLAC) is the topic of this paper. Bunch current monitors are used to measure the charge in the electron/positron bunches traveling in particle storage rings. The BCMs in the PEP-II storage rings need to be upgraded because components of the current system have failed and are known to be failure prone with age, and several of the integrated chips are no longer produced making repairs difficult if not impossible. The main upgrade is replacing twelve old (1995) field programmable gate arrays (FPGAs) with a single Virtex II FPGA. The prototype was tested using computer synthesis tools, a commercial signal generator, and a fast pulse generator. 


\section{INTRODUCTION}

PEP-II is a pair of $2.2 \mathrm{~km}$ circumference synchrotron storage rings for electrons and positrons with a standard operating current on the order of two amps located at the Stanford linear accelerator center (SLAC). Charged particles are injected into the ring from the linear accelerator at a frequency of up to $60 \mathrm{~Hz}$; actual injection is controlled using data from the BCM. The ring traps charged particles using bend magnets to steer particles around the ring and quadrapole magnets to focus the beam. The energy radiated due to acceleration of the stored particles must be replaced. PEP-II replaces the lost energy by using cavities filled with $476 \mathrm{MHz}$ microwave radiation to produce a stable orbit in the ring. Positrons and electrons are propagated in separate rings in opposite directions. The two rings are stacked on top of each other and intersect at a single interaction point.

PEP-II has 3492 buckets per ring for charge bunches to travel in. A bucket is a phase of the $476 \mathrm{MHz}$ microwave where charged particles can be pulled around the ring without decaying to the center or being propelled out of the ring. Particles that are out of synchronous phase will either relax into phase or be completely ejected from the beam. This self correcting effect is a result of the stability of the bucket position. If a particle is out of synchronous phase it will not receive the proper amount energy from the microwave to achieve synchronous orbit.

If a particle is too late compared to the synchronous phase; it will gain less than the energy required to replace the radiated energy, this results in a net loss of energy. The less energetic particle will be bent by the bend magnets slightly more than during the previous turn. The lower energy particle will take a shorter path around the ring and arrive earlier than the previous turn, since the particles are propagated at approximately the speed of light. Thus the high energy(late) particle arrives earlier and earlier with each turn and are relaxed toward the synchronous phase. The result is an oscillation between too early in phase(low energy) and too late in phase(high energy). This oscillation is a product of the 
relaxation of the high/low energy particles and is a stable of form particle propagation inside a synchrotron.

Every other bucket position is occupied with a charge bunch, where a charge bunch is group of charged particles moving around the ring in the same bucket. Every other bucket is left empty in an effort to minimize parasitic collisions between the positron and electron beams. A parasitic collision is when an electron bunch nearly collides with a positron bunch, resulting in a beam deflection at the interaction point. The last forty buckets are left empty in case the beam has to be terminated unexpectedly. The beam is terminated by turning on a magnet that bends the beam off course into a beam dump. The magnet field requires a finite amount time to reach the critical bend value. The forty bucket space is large enough so that the magnetic field can reach the critical strength to completely bend the beams into the beam dumps. If the magnetic field does not reach the critical value, the beam will oscillate, making a potentially bad situation worse.

Bunch current monitoring is a useful technique for fast measure and respond scenarios with semi-periodic subjects specifically in synchrotrons. BCMs are used to monitor the electron bunches and positron bunches traveling in the PEP-II particle storage rings at SLAC. In PEP-II charge bunches pass by the detector every 4.2 ns. Synchronous phase change from bunch to bunch occurs because the microwave cavities fill up with slightly more energy during the forty bucket gap, and so bunches just after the gap are slightly late compared to the synchronous phase, while bunches at the end of the bunch train are slightly early. In order to gather useful data the system must gather and respond to data very quickly. Particles are injected from the linear accelerator to ensure optimum charge density per bunch, and injection timing is determined by the charge information gathered from the BCM.

The BCM detector outputs a signal dependent on the electric field of the particle beam. The electrons/positrons are highly relativistic so the electric field detected results in a very fast bipolar pulse. The output of the detector is from four electric field sensors, and the 
outputs are fed to a comb filter that combines the fast pulses (100 ps) into a single two period sinusoid of $1428 \mathrm{MHz}$. The sinusoid is then mixed with a $1428 \mathrm{MHz}$ reference signal that is stepped through four ninety degree phases. The signal is fed through a low pass filter, and then is digitized by the on board digitizer. The four phase technique allows extraction of the bunch current value. The bunch current value is found by taking the sum of the squares of the difference between two signal values out of phase by 180 degrees. The average value is then computed over many turns to smooth out noise in the measurement. The mathematics is as follows.

The output of the comb filter is

$$
s(t)=s_{0} \cos \left(\omega t+\phi_{\text {beam }}\right)
$$

where $s_{0}$ is the bunch current. The output of the mixer is

$$
\mathrm{DC}+s_{0} V_{0} \cos (\omega t) \cos \left(\omega t+\phi_{\text {beam }}+\phi_{\text {step }}\right),
$$

where $V_{0}$ is the reference amplitude. If we add the trigonometric identities

$$
\cos (2 \omega t+\phi)=\cos (\omega t) \cos (\omega t+\phi)-\sin (\omega t) \sin (\omega t+\phi)
$$

and

$$
\cos (\phi)=\cos (\omega t) \cos (\omega t+\phi)+\sin (\omega t) \sin (\omega t+\phi)
$$

we can rewrite the output of the mixer as

$$
\mathrm{DC}+\frac{s_{0} V_{0}}{2} \cos \left(\phi_{\text {beam }}+\phi_{\text {step }}\right)(\cos (2 \omega t)+1) .
$$


If we pass this output through a low pass filter, the signal becomes

$$
\frac{s_{0} V_{0}}{2} \cos \left(\phi_{\text {beam }}+\phi_{\text {step }}\right)+\mathrm{DC} \text {. }
$$

The step phase $\phi_{\text {step }}$ can assume four discrete values, $0, \pi / 2, \pi$ and $\frac{3 \pi}{2}$. If we superpose the 4 values of the signal, evaluated for the four different values of $\phi_{s t e p}$, we get

$$
s_{0} V_{0}=\sqrt{\left(\frac{s_{0} V_{0}}{2} \cos \left(\phi_{\text {beam }}\right)+\frac{s_{0} V_{0}}{2} \cos \left(\phi_{\text {beam }}\right)\right)^{2}+\left(-\frac{s_{0} V_{0}}{2} \sin \left(\phi_{\text {beam }}\right)-\frac{s_{0} V_{0}}{2} \sin \left(\phi_{\text {beam }}\right)\right)^{2}}
$$

A field programmable gate array (FPGA) chip is an array of thousands to millions of logic cells integrated on to a single chip driven by an external clock. The logic cells are controlled by semiconductor switches. The switch states are defined on an onboard PROM (programmable read only memory). The switch states control how the cells are linked, and how they function. The PROM is programmed using a hardware description language. The current FPGAs (1995) are too slow to handle the required $476 \mathrm{MHz}$ processing rate, significant signal conditioning is required before data can be gathered. The signal from the BCM detector is connected to a VXI crate with two digitizers and two decimation boards. A VXI crate is a chassis in which other electronics can be implemented; the crate used has a built in CPU and power supply. The even buckets are digitized by one of the two digitizers and the odd buckets are digitized by the other. The digitizer sends the digitized signal to one decimation board with six FPGAs. The data stream to the FPGAs is decimated by eight; i.e., by sampling one out of every eight passes a charge bunch made so that $7 / 8$ of the data is lost. The proposed system does not require such decimation and can gather all of the data using two boards; one board with a digitizer and an FPGA, a second board with a CPU running Linux, a digitizer, a power supply, a hard disk, a USB interface, and dual port RAM. For further information on the current system please read[1]. 


\section{MATERIALS AND METHODS}

The proposed upgrade plan replaces the VXI crate in each ring with the the new digitization board and the Linux computer board. In short the VXI crates contain twelve FPGAs from 1995 and the new system contains one modern FPGA (Virtex II). The new FPGA will be programmed using Verilog Hardware Description Language (HDL) in the Xilinx synthesis environment.

Verilog HDL is the language used to program the Virtex-II FPGA component of the BCM in the PEP-II storage rings at SLAC. Verilog HDL is a structure for constructing routines that can be implemented onto programmable hardware. The Xilinx ISE 8.1i synthesis environment has an implementation tool that writes the Verilog routines to the switches on the FPGA PROM. ModelSim XE III 6.0d is the program used to simulate how the Verilog routines will be implemented on the FPGA and how the FPGA will operate once programmed.

FPGAs represent a fast and inexpensive method of prototyping and implementing design. Programming is easier and less expensive to alter than a soldered circuit board. The reprogrammable nature of the FPGA also improves the cost effectiveness of the chip. One FPGA can be used in several applications by implementing different HDL routines. FPGAs can be programmed into sections that act independently of each other. The Virtex-II FPGA is replacing twelve older FPGAs by programming the FPGA into six different sections. Each section is programmed as a single small FPGA and runs in parallel with the other sections. The new FPGA will total up the signal from many turns, the computer will use that sum to calculate the value of equation [7].

\section{DISCUSSION AND CONCLUSIONS}

The prototype was tested with various computerized test benches and signal generators. The prototype showed that the proposed system is feasible and can be implemented, however 
further devlopment is required before the system is ready for application. The proposed system is a Linux computer operating all of the required components to collect and process data from the BCM detector. The Linux computer will be directly interfaced with the SLAC network. Figure 1 compares the proposed system to the current system.

\section{REFERENCES}

[1] M.J Chin, J.A. Hinkson, PEP-II Bunch-by-Bunch Current Monitor, Particle Accelerator Conference, Vancouver B.C. , Canada, May 12-14 1997, pg 2235-2237 


\section{FIGURES}

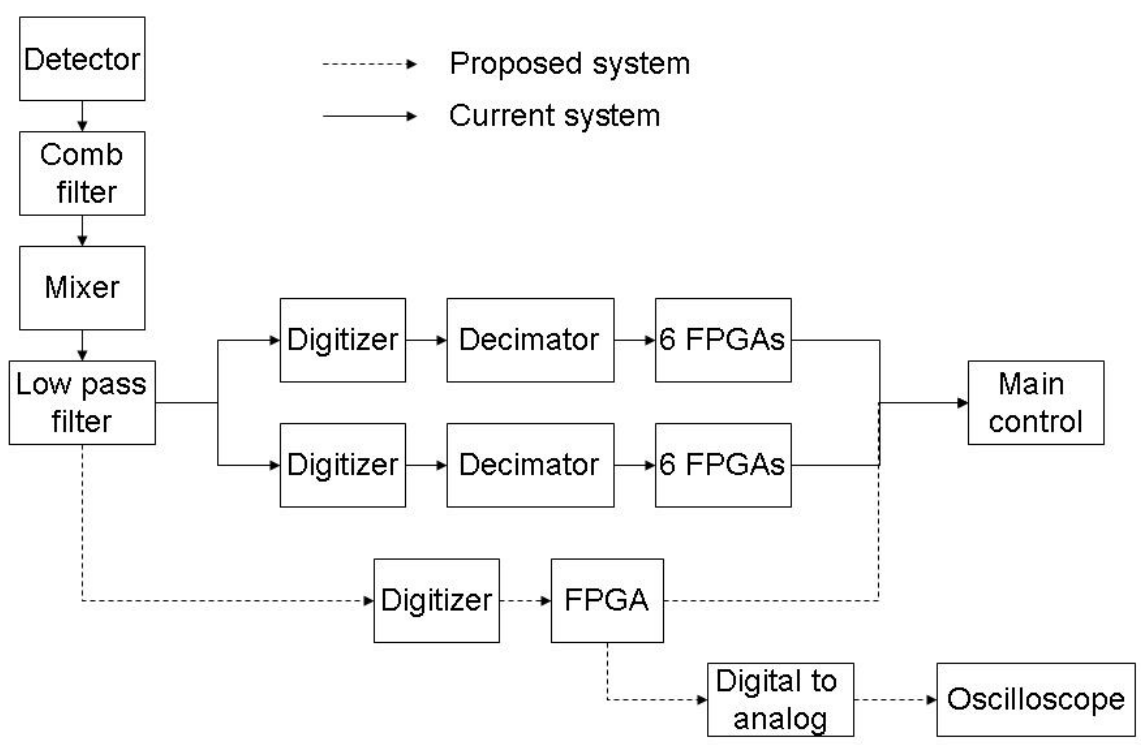

Figure 1: A diagram comparing the old system to the proposed system. The proposed system replaces 6 FPGAs with one and does not need a decimator. 


\title{
X-ray photoelectron spectroscopy of $\mathrm{GaP}_{1-\mathrm{x}} \mathrm{N}_{\mathrm{x}}$ photocorroded as a result of hydrogen production through water electrolysis
}

\author{
Marie A. Mayer \\ Office of Science, Science Undergraduate Laboratory Internship (SULI) \\ University of Illinois at Urbana-Champaign \\ Stanford Synchrotron Radiation Laboratory at the Stanford Linear Accelerator Center \\ Menlo Park, CA
}

August 18, 2006

Prepared in partial fulfillment of the requirements of the Office of Science, Department of Energy’s Science Undergraduate Laboratory Internship under the direction of A. Nilsson in the Stanford Synchrotron Radiation Laboratory at Stanford Linear Accelerator Center.

Participant:

Signature

Research Advisor:

Signature 


\section{Table of Contents}

Abstract $\quad 2$

1. Introduction 3

2. Materials and Methods 5

3. Results 8

4. Discussion and Conclusions 9

5. Acknowledgements 10

6. References 11

8. Figures 13 


\begin{abstract}
$\mathrm{X}$-ray photoelectron spectroscopy of $\mathrm{GaP}_{1-\mathrm{x}} \mathrm{N}_{\mathrm{x}}$ photocorroded as a result of hydrogen production through water electrolysis. MARIE A. MAYER (University of Illinois at Urbana-Champaign, Urbana, IL 61801) ANDERS NILSSON (Stanford Linear Accelerator Center, Menlo Park, CA, 94025).
\end{abstract}

Photoelectrochemical (PEC) cells produce hydrogen gas through the sunlight driven electrolysis of water. By extracting hydrogen and oxygen from water and storing solar energy in the $\mathrm{H}-\mathrm{H}$ bond, they offer a promising renewable energy technology. Addition of dilute amounts of nitrogen to III-V semiconductors has been shown to dramatically increase the stability of these materials for hydrogen production. In an effort to learn more about the origin of semiconductor photocorrosion in PEC cells, three samples of p-type GaP with varying levels of nitrogen content ( $0 \%, 0.2 \%, 2 \%)$ were photocorroded and examined by X-ray Photoelectron Spectroscopy (XPS). GaPN samples were observed to be more efficient during the hydrogen production process than the pure GaP samples. Sample surfaces contained gallium oxides in the form of $\mathrm{Ga}_{2} \mathrm{O}_{3}$ and $\mathrm{Ga}(\mathrm{OH})_{3}$ and phosphorus oxide $\left(\mathrm{P}_{2} \mathrm{O}_{5}\right)$, as well as surface oxides from exposure to air. A significant shift in intensity from bulk to surface peaks dramatic nitrogen segregation to the surface during photoelectrochemical hydrogen production. Further investigations, including using a scanning electron microscope to investigate sample topography and inductively coupled plasma mass spectroscopy (ICP-MS) analysis for solution analyses, are under way to determine the mechanism for these changes. 


\section{INTRODUCTION}

\subsection{Background}

As fuel costs increase and supplies become increasingly limited, the demand for alternative energy sources rises. The fuel consumption of the United States in 2004 consisted of $94 \%$ fossil fuels, with only $1 \%$ of renewable energy originating from sunlight [1]. However, since the sun produces the equivalent of 1.3 trillion barrels of oil in a year; the remaining challenge is how to harvest this energy. [2] Photoelectrochemical (PEC) cells produce hydrogen gas through the sunlight driven electrolysis of water. By extracting hydrogen and oxygen from water and storing solar energy in the $\mathrm{H}-\mathrm{H}$ bond, they offer a promising renewable energy technology. This technology has provided energy conversion efficiencies of up to $12.4 \%$ using multijunction regenerative solar cells [3], but suffers due to instability from photocorrosion.

A PEC cell has a typical electrochemical cell setup (Figure 1.1), with a p-type semiconductor cathode and a metal anode (or vice versa in the case of an n-type semiconductor). Both electrodes are immersed in electrolyte, which for hydrogen production would be water or a slightly acidic solution to increase the count of hydronium ions. The semiconductor-electrolyte interface forms the Helmholtz layer, which causes band bending. A further discussion of the physics of the semiconductors is given by Bak et al. [2]. When illuminated by sunlight, optically-excited holes diffuse to the bulk of the p-type semiconductor, while the minority carrier electrons travel to the semiconductor-electrolyte interface. The electrons reduce hydrogen contained in water molecules according to:

$$
2 \mathrm{H}_{2} \mathrm{O}+2 \mathrm{e}^{-} \rightarrow \mathrm{H}_{2}+2 \mathrm{OH}^{-}
$$

The holes travel through an electrical connection from the cathode to the anode, or oxidation site:

$$
2 \mathrm{H}_{2} \mathrm{O}+4 \mathrm{~h}^{+} \rightarrow \mathrm{O}_{2}+4 \mathrm{H}^{+}
$$


Since the gases are generated at different electrodes, they are easily separable. The $\mathrm{H}_{2}$ is retained as fuel which, when used in a fuel cell to generate electricity, recombines with oxygen in the air to produce water and heat as the only byproducts, completing the pollution-free renewable energy cycle.

\subsection{Materials Requirements}

The reaction requires a band gap of approximately $2 \mathrm{eV}: 1.23 \mathrm{eV}$ required for completion of reactions 1.1 and 1.2, plus an additional voltage wasted on overpotentials [2]. The semiconductor chosen must also be conducive to absorbance of solar energy. A smaller band gap is ideal to absorb the maximum number of photons. Additionally, the valence band of the semiconductor must be more positive than that of the oxidation electropotential and the conduction band more negative than the reduction electropotential on an electrochemical scale (Figure 1.2). If a single-junction system is under consideration, few materials have a band gap that efficiently appeases both the electrolysis and efficient solar energy conditions.

There is also the issue of corrosion, or decomposition of the semiconductor, to consider. The greater the damage to the semiconductor surface, the less functional the cell. Oxidation (Equation 1.3) can dislodge metal ions from the semiconductor surface, while reduction (Equation 1.4) of the semiconductor can result in the formation of a thin layer of metal on the semiconductor surface.

$$
\begin{aligned}
& M X+z^{+}+\text {solvent } \rightarrow M_{\text {solvent }}^{z+}+X \\
& M X+z e^{-}+\text {solvent } \rightarrow M+X_{\text {solvent }}^{z-}
\end{aligned}
$$

Corrosion is dependent upon the thermodynamic and kinetic stability of the sample, which is, on a simple level, observable in terms of the values of corrosion potentials with respect to hydrolysis potentials. For corrosion to occur, the electropotentials associated with the corrosion 
reactions would need to lie between the oxidation and reduction electropotentials for hydrolysis, as well as outside of the semiconductor band edges if the sample is under illumination (Figure 1.3). [4]

While there are a number of ways to address the band alignment and corrosion, alloying has produced positive effects on both accounts. In the GaP system, the effect of nitrogen on the band gap of these materials is twofold: nitrogen shifts the band gap transition toward direct alignment; and it shifts the band edges to a more favorable position compared to the water red-ox electropotentials. Nitrogen content up to approximately $2 \%$ has been shown to decrease corrosion, but at higher concentrations, the stability of the sample is threatened. The addition of nitrogen adds resistance to chemical attack, but the exact nature of the influence on photocorrosion is an open question. [5]

As an effort to learn more about the photocorrosion of p-type semiconductors in PEC hydrogen production, three samples of $\mathrm{GaP}_{\mathrm{x}} \mathrm{N}_{1-\mathrm{x}}$ (band gap of $2.25 \mathrm{eV}$ [6]) with varying amounts of infused nitrogen $(\mathrm{x}=0,0.002,0.02)$ were photocorroded and examined by synchrotron X-ray photoelectron spectroscopy (XPS). The objective included probing the surface before and after corrosion to learn about the causes of corrosion and effects of nitrogen. The possibilities of energy conversion with renewable resources depend upon the stability of the materials available.

\section{MATERIALS AND METHODS}

$\mathrm{GaP}_{1-\mathrm{x}} \mathrm{N}_{\mathrm{x}}(\mathrm{x}=0,0.02,0.002)$ samples were grown at the National Renewable Energy Laboratory (NREL) by metal-organic chemical vapor deposition (MOCVD). Samples were cleaved into two pieces; one piece was reserved as an uncorroded standard, while the remaining piece of each was photocorroded. 


\subsection{Electrochemical Corrosion}

In order to accommodate acidic and vacuum environments, electrochemistry was performed using a mechanical cell made of Pyrex and Teflon (Figure 2.1). This cell exposed only the surface of the semiconductor to the solution, and the back contact was electrically connected to the potentiostat. The platinum counter electrode and Ag/AgCl reference electrodes were inserted as shown. Photocorrosion was done using a Gamry PHE200 potentiostat.

The Gamry chronopotentiometry program was used to record current vs. time and potential vs. time. The $\mathrm{GaP}_{1-\mathrm{x}} \mathrm{N}_{\mathrm{x}}$ samples were etched with concentrated sulfuric acid, then immersed in a deaerated solution of 3M sulfuric acid, along with a Ag/AgCl reference electrode and a platinum counter electrode. An optical lamp was focused on the sample for the duration of corrosion. In order to evolve hydrogen under $100 \mathrm{~mW} / \mathrm{cm}^{2}$ (1 sun) conditions, a cathodic current of $1.57 \mathrm{~mA}$ was passed through the sample for 8 hours, resulting in the charge passage of $45.2 \mathrm{C}$. Samples were stored in vacuum, disregarding transport times. The solution was retained for inductively coupled plasma mass spectroscopy (ICP-MS) analysis.

During hydrogen production, a qualitative examination revealed that the $\mathrm{GaP}_{0.98} \mathrm{~N}_{0.02}$ sample, which showed the most visible surface change, was the most effective at reducing the solution for the entire 8 hours. Bubbles from the $\mathrm{GaP}_{0.998} \mathrm{~N}_{0.002}$ sample appeared, but had decreased in number after 8 hours. GaP showed few obvious bubbles during hydrogen production, although there was a slight surface change.

\subsection{XPS Measurements}


Core level spectroscopy was used to study the surfaces of the corroded $\mathrm{GaP}_{1-\mathrm{x}} \mathrm{N}_{\mathrm{x}}$ samples. X-ray photoelectron spectroscopy (XPS) was performed using synchrotron x-ray radiation at the Stanford Synchrotron Radiation Laboratory (SSRL) on beamline 5-2. The x-ray energy used set the kinetic energies of the released electrons according to:

$$
E_{k i n}=h v-E_{B}-\Phi[7]
$$

Here, $h v$ is the energy of the x-ray photon, $E_{B}$ is the binding energy of the electron and $\Phi$ is the work function of the analyzed sample. Since the x-ray energy is tuneable and the work function is fixed, the binding energy reveals information about the surface structure: elements present and their nearest neighbors, as well as binding structures.

The structure of the corroded surface can change as a function of depth. A depth profile can collected by measuring spectra at different photoelectron kinetic energies, due to the relationship between the mean free path of an electron and its kinetic energy, as shown in Figure 2.2. In synchrotron-based XPS, the kinetic energy of a photoelectron can be altered by changing the x-ray photon energy. Therefore, a given x-ray photon energy will emit an electron of a particular element with a specific mean free path.

$$
\Delta=\lambda_{i} \cos (\alpha)[8]
$$

Equation 2.2 shows that the mean escape depth $(\Delta)$ is related to the mean free path $\left(\lambda_{\mathrm{i}}\right)$ through the emission angle of the electron. Therefore, by equation 2.2, the elements present at a specific depth can be monitored as a method to compare the oxide throughout the surface layers.

XPS was measured for six samples, as grown (at NREL by MOCVD) and a corroded sample of each $\mathrm{GaP}_{1-\mathrm{x}} \mathrm{N}_{\mathrm{x}}(\mathrm{x}=0,0.002,0.02)$ In order to calibrate energies, gold deposited on $\mathrm{Si}$ was placed between the samples, and the $\mathrm{Au}_{4 \mathrm{f}^{7} / 2}$ core level was measured as a reference. Depth 
profiles of the samples were obtained by measuring the core level peaks for the elemental species (Ga3d, Ga3p P2p, N1s) and native surface oxides (O1s and C1s).

Energies were specifically chosen to avoid Auger peaks. Measurements were taken at photon energies adjusted according to the binding energies of a given species (Eqn. 2.1) to give emitted electrons kinetic energies of 200, 450, 750 and $900 \mathrm{eV}$. These measurements equate to escape depths (assuming grazing incidence angle) of 9, 14.85, 21.25, and $24.3 \AA$ respectively [9].

\section{RESULTS}

\section{XPS Measurements}

XPS results are distinguishable based upon two variables: nitrogen content and corrosion treatment of the samples. A number of features are present and worthy of note, but a limited selection will be presented here.

In all cases, there were a number of oxides present on the samples, generally seen at binding energies higher than the pure species. The Ga3d spectrum is shown as an example, with the oxides denoted (Figure 3.1). The intensity of these oxides relative to the species measured decreases at higher energies.

The Ga3d spectra at all energies show a decrease in gallium content near the surface after corrosion (Figure 3.2).

At all energies, the uncorroded nitrogen spectra for the $\mathrm{GaP}_{1-\mathrm{x}} \mathrm{N}_{\mathrm{x}}(\mathrm{x}=0.02)$ sample shows two peaks, one corresponding to $\mathrm{N}$ bonded to $\mathrm{Ga}$ in the bulk, the other to $\mathrm{NO}_{\mathrm{x}}$ and $\mathrm{NH}_{\mathrm{x}}$ on the surface. The bulk peak is higher in binding energy by approximately $2.5 \mathrm{eV}$ (Figure 3.3), while the second, smaller peak does not appear in the corroded sample spectra. These features are also visible in the $\mathrm{GaP}_{1-\mathrm{x}} \mathrm{N}_{\mathrm{x}}(\mathrm{x}=0.002)$ samples. 


\section{DISCUSSION AND CONCLUSIONS}

Qualitatively, the samples showed agreement with previous literature: the addition of nitrogen improves the ability of the GaP to produce hydrogen. The XPS data supports this by showing consistent differences between nitrogen containing samples and the GaP.

The large oxide peak shows evidence of oxides observed in a previous study of GaAs, which, as another III-V compound, behaves similarly to GaP. C.C. Surdu-Bob et al. report the binding energy of $\mathrm{Ga}_{2} \mathrm{O}_{3}$ at $20.7 \mathrm{eV} \pm 0.1 \mathrm{eV}, \mathrm{Ga}_{2} \mathrm{O}$ at 20.1 and $\mathrm{Ga}(\mathrm{OH})_{3}$ at $21.6 \mathrm{eV}$. [10] Thus, the gallium oxides (denoted on Figure 3.1) were most likely in the forms $\mathrm{Ga}_{2} \mathrm{O}, \mathrm{Ga}_{2} \mathrm{O}_{3}$ and $\mathrm{Ga}(\mathrm{OH})_{3}$. The oxide on phosphorus appeared in a peak centered at a binding energy of approximately $133 \mathrm{eV}$, which matches $\mathrm{P}_{2} \mathrm{O}_{5}$ (binding energy of approximately $133.6 \mathrm{eV}$ ).

The gallium-poor surface after corrosion is significant in determining the corrosion mechanism. A proposed corrosion mechanism in agreement with the Ga depletion is:

$$
\mathrm{GaP}+3 \mathrm{H}^{+} \rightarrow \mathrm{Ga}^{3+}+\mathrm{PH}_{3}
$$

The depletion of surface Ga matches with both mechanisms. However, the corrosion solutions are being submitted for inductively coupled plasma mass spectroscopy (ICP-MS) analysis to identify the species present.

The disappearance of the second nitrogen peak in GaPN samples (Figure 3.3) indicates the migration of nitrogen from the bulk toward the sample surface as a result of corrosion. Based upon binding energies, the smaller peak at lower binding energy indicates nitrogen added to the sample during MOCVD, or “bulk” nitrogen. The larger peak represents nitrogen oxidized by exposure to air while mounting them on the sample holder. Since the bulk peak is not apparent in the corroded spectra taken at the same photon energy, the conclusion is that the bulk nitrogen 
migrated to the sample surfaces during corrosion. One possible explanation for the surface nitrogen is the formation of GaN (band gap of approximately 3.2) during corrosion. Since GaN has a larger band gap, it has a tendency to be more resistant to corrosion. In this case, the semiconductor would actually be behaving like a tandem cell: the smaller band gap of GaP for maximum solar absorbance, but the larger band gap adding stability. The GaN may be undetectable currently, as the sample oxidized after removal from the electrolyte. But, as there are a number of electronic consequences of nitrogen implantation, investigations are currently underway to make final conclusions as to the significance of the nitrogen migration.

In future analysis, ICP-MS analysis will detect the presence of any species desorbed from the surface of the $\mathrm{GaP}_{1-\mathrm{x}} \mathrm{N}_{\mathrm{x}}$ during corrosion. These may have been produced either directly from the semiconductor reactions, or as a result of interaction with the acidic solution. The results of these tests should indicate what species form during corrosion. The samples have also been submitted to view topography with a SEM or TEM. Hopefully, analysis of the semiconductor surface combined with knowledge of the solution composition will yield a complete set of corrosion products from which the reaction mechanisms can be deduced. By learning about the surface, it may be possible to eventually engineer the surface of the semiconductors to increase the lifetime of a hydrogen producing PEC.

\section{ACKNOWLEDGEMENTS}

This research was conducted at the Stanford Synchrotron Radiation Laboratory. Thanks first to Theanne Schiros for her undying enthusiasm for the project and the time she spent teaching me

this summer. Thank you to Jen Leisch, whose constant flux of wisdom kept the project moving. I would also like to thank Theanne and Jen for a constant flow of food, and Trader Joe's for 
producing said food. I gratefully acknowledge the many hours that Lars-Åke Näslund spent at the beamline with us instead of sleeping, the patience and dedication of Anton Nikiton, the pearls of wisdom provided by Hirohito Ogasawara, the general helpfulness of Mike Toney and the constant assistance from Experimental Support. Thank you also to everyone who even so much as answered one of my numerous questions or caused me to smile. Finally, we thank Anders Nilsson for his guidance and his efforts to introduce such renewable energy projects at the Stanford Linear Accelerator Center.

\section{REFERENCES}

[1] Energy Information Administration, 2004, http://www.eia.doe.gov/.

[2] T. Bak et al., "Photoelectrochemical hydrogen generation from water using solar energy. Materials Related Aspects,” International Journal of Hydrogen Energy, vol. 27, pp 991 1022, 2002.

[3] J. Leisch. CuInSe ${ }_{2}$ and Related Alloy Thin Film Semiconductors for Photoelectrochemical Hydrogen Production, PhD Thesis, Golden, Colorado, 2006.

[4] H. Gerischer, "On the stability of semiconductor Electrodes against photodecomposition,” Journal of Electroanalytical Chemistry, vol. 82, pp133-143, 1977.

[5] T. Deutsch. Sunlight, Water and III-V Nitrides for Fueling the Future, PhD Thesis, Boulder, Colorado, 2006.

[6] W. D. Callister. Materials Science and Engineering: An Introduction, Fourth Edition, New York: John Wiley and Sons, pp 603, 1997. 
[7] N. Martensson and A. Nilsson, "High-Resolution Core-Level Photoelectron Spectroscopy of Surfaces and Adsorbates," in Applications of Synchrotron Radiation, W. Eberhardt, Ed. Berlin: Springer-Verlag, pp. 65-125, 1995.

[8] P. Pianetta, "Low-energy electron ranges in matter," in Center for X-ray Optics and Advanced Light Source: X-ray Data Booklet, Berkeley: Lawrence Berkley National Laboratory, p 3-11, 2001.

[9] S. Tanuma et al., "Calculations of Electron Inelastic Mean Free Paths: III. Data for 15 Inorganic Compounds over the 50-2000 eV Range,’” Surface and Interface Analysis, vol 17, pp. 927, 1991.

[10] C.C. Surdu-Bob et al., “An X-Ray photoelectron spectroscopy study of the oxides of GaAs,” Applied Surface Science, vol. 183, pp 126-136, 2001. 


\section{FIGURES}

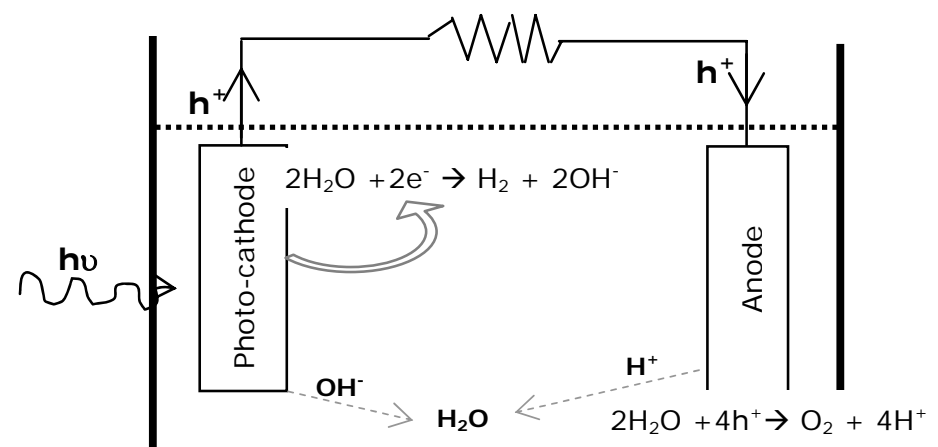

Figure 1.1. Photo-electrochemical cell arrangement for electrolysis

(-)

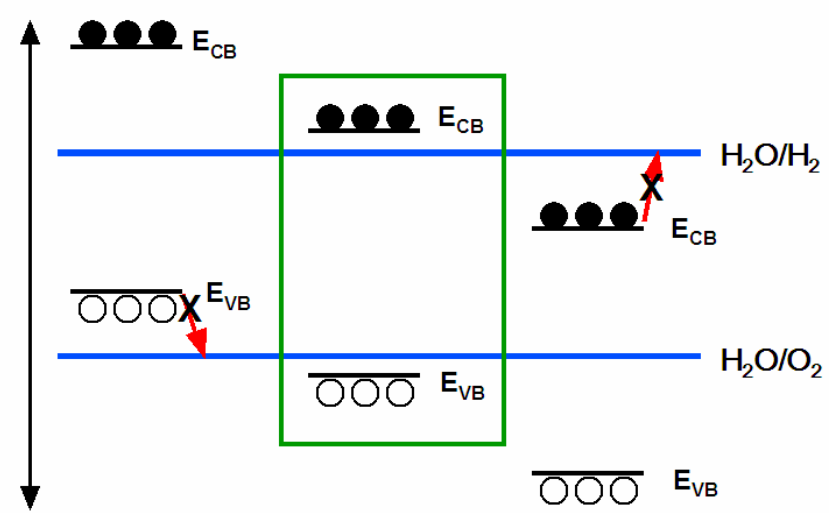

$(+)$

Figure 1.2. Band edge position requirements for a PEC semiconductor material. The conduction band $\left(\mathrm{E}_{\mathrm{CB}}\right)$ and valence band $\left(\mathrm{E}_{\mathrm{VB}}\right)$ energies must straddle the water redox potentials. Figure from Leisch [3].

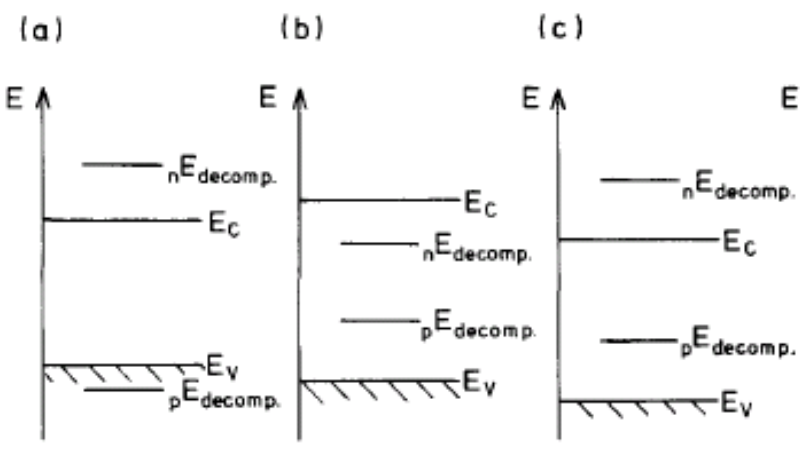

Figure 1.3. Typical correlations between energy positions of band edges and decomposition potentials, controlling stability against photodecomposition. (a) Stable, (b) unstable, (c) stable against cathodic decomposition. Figure from Gerischer [4]. 


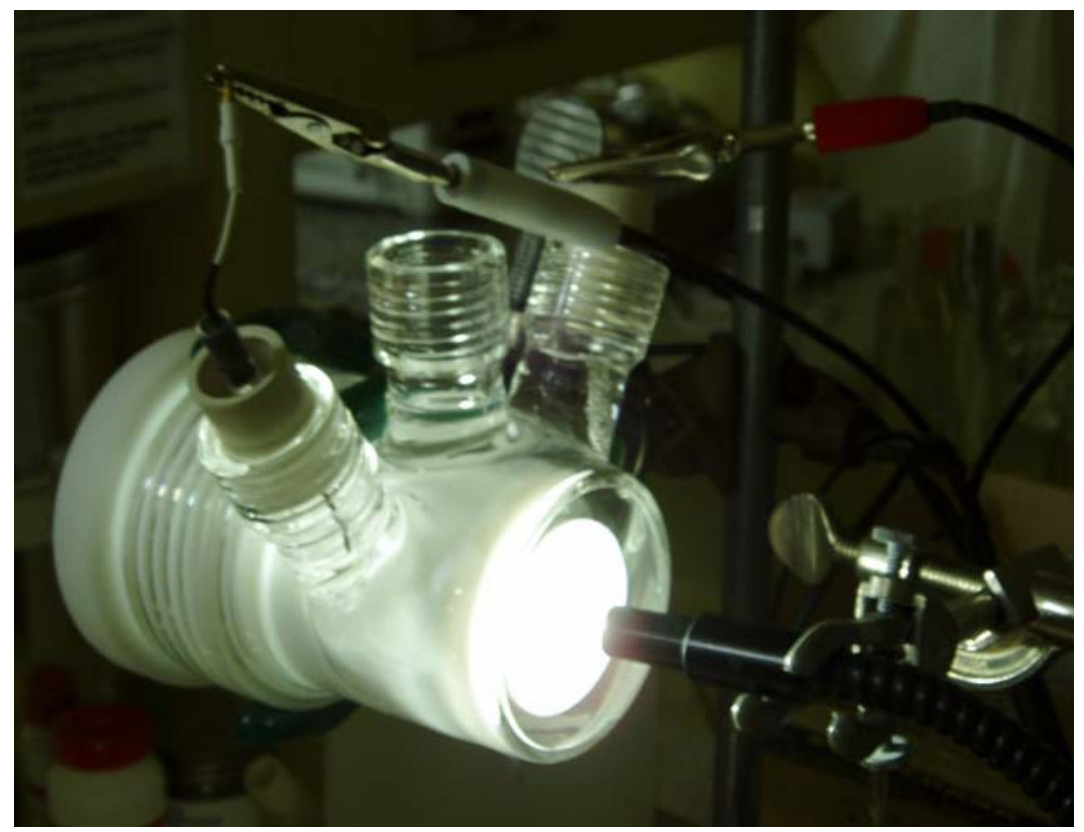

Figure 2.1. NREL electrochemical cell made of Pyrex and Teflon.

Semiconductor directly behind light, Pt counter and $\mathrm{Ag} / \mathrm{AgCl}$ reference electrodes used.

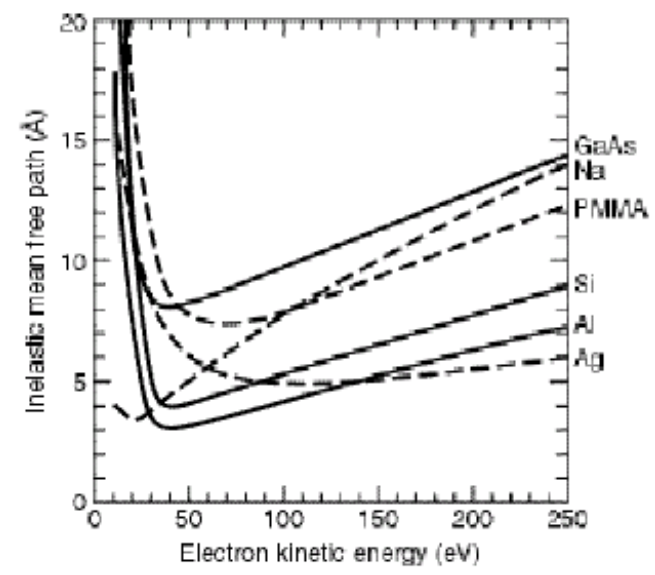

Figure 2.2. Inelastic mean free path as a function of core electron kinetic shown for six different materials. [9] 


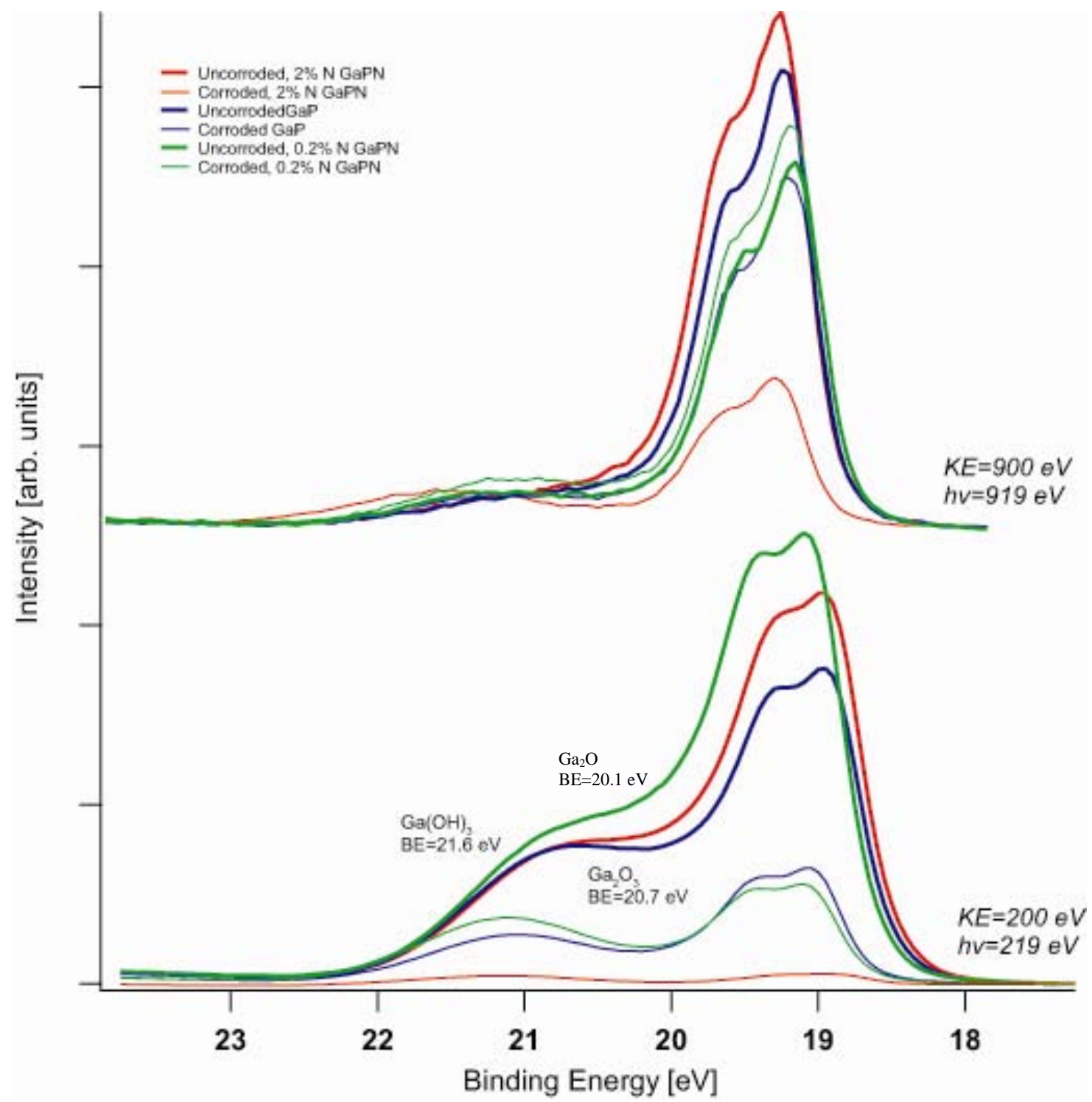

Figure 3.1. Ga3d spectra at photon energies of $219 \mathrm{eV}$ and $919 \mathrm{eV}$. Suspected oxides are labeled on the lower energy spectrum. The intensities of the oxides is much weaker at higher energies. 


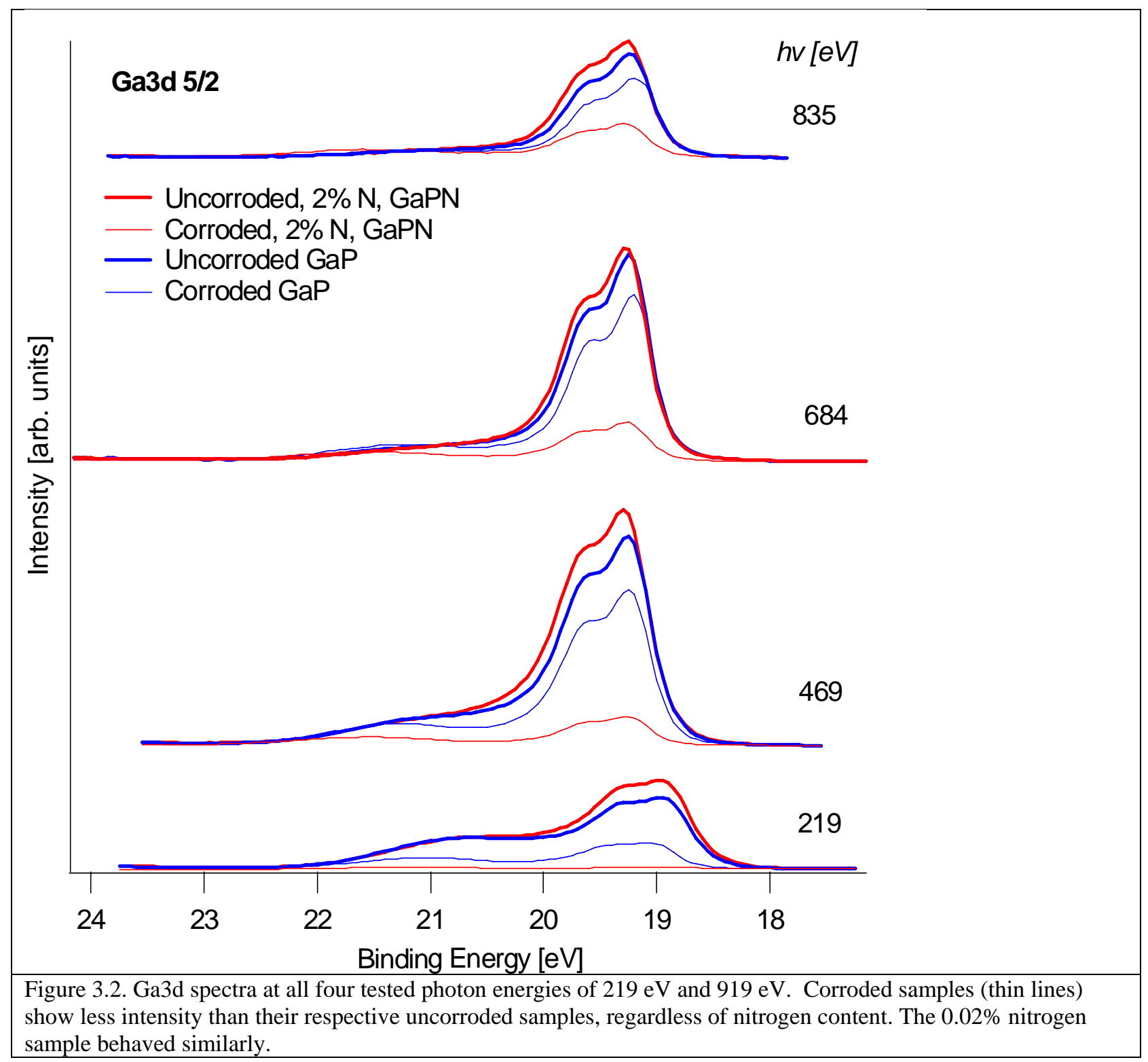




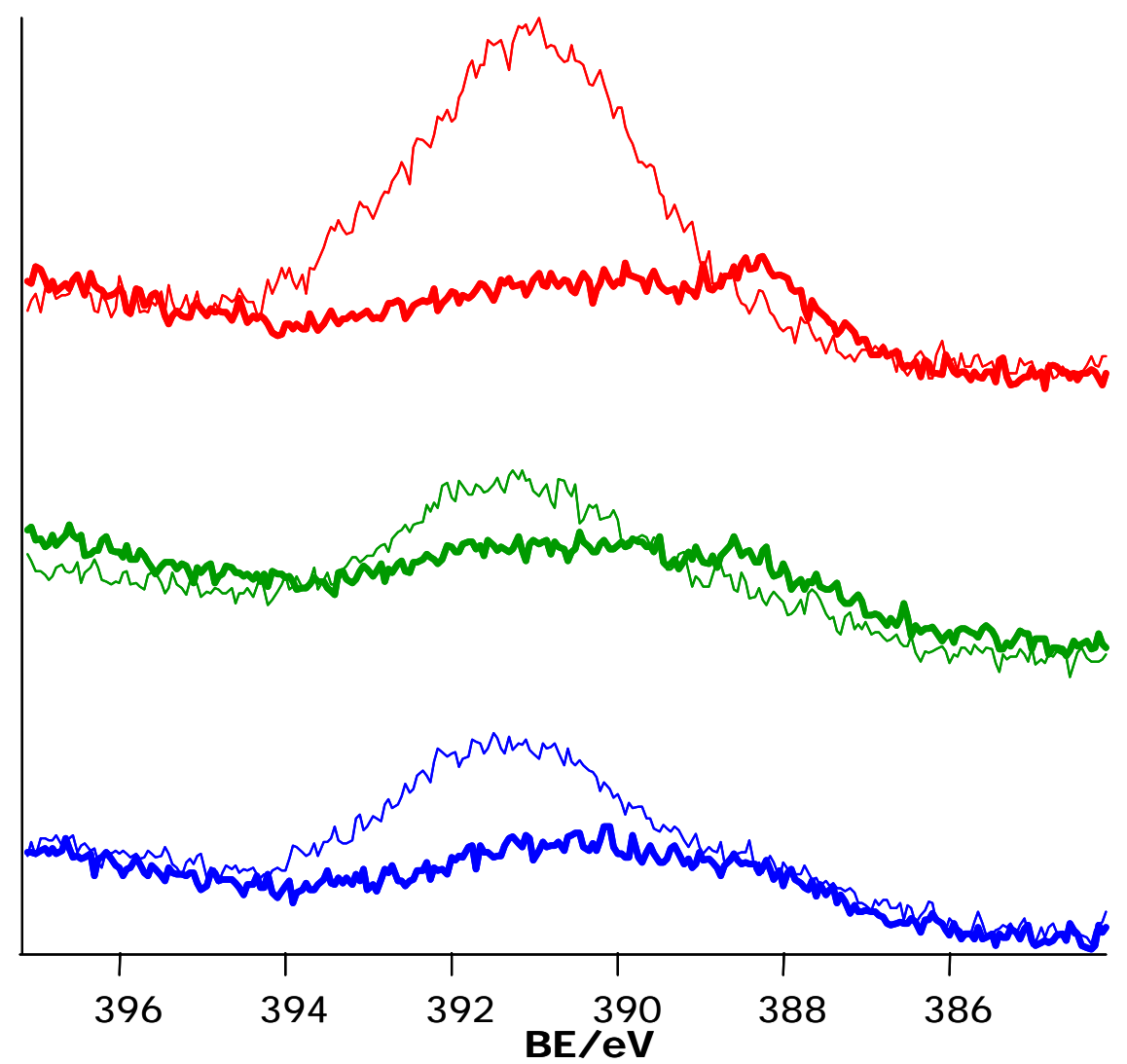

Figure 3.3. N1s spectra for $\mathrm{GaP}$ and $\mathrm{GaPN}_{0.02}$ as a function of intensity. Bulk nitrogen (maximum at $397 \mathrm{eV}$ ) is apparent in the uncorroded sample, but not in the sample after corrosion. The GaP samples only show nitrogen surface contamination (maximum at $400 \mathrm{eV}$ ), before and after corrosion. 